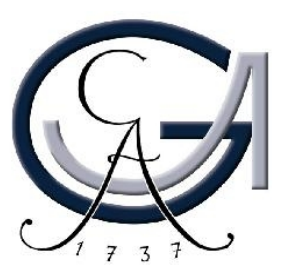

\title{
The function of
}

Nup358 in

\section{nucleocytoplasmic transport}

\author{
Dissertation \\ for the award of the degree \\ „Doctor rerum naturalium“ \\ Division of Mathematics and Natural Sciences \\ of the Georg-August University Goettingen
}

submitted by

Sarah Wälde

from Landshut

Goettingen 2010 
Member of the Thesis Committee (First Reviewer):

PD Dr. Ralph H. Kehlenbach

Department of Biochemistry I

Center for Biochemistry and Molecular Cell Biology

Georg-August University, Goettingen

Member of the Thesis Committee (Second Reviewer):

Prof. Dr. Reinhard Lührmann

Department of Cellular Biochemistry

Max Planck Institute for Biophysical Chemistry, Goettingen

Member of the Thesis Committee:

Prof. Dr. Ralf Ficner

Department of Molecular Structural Biology

Goettingen Center for Molecular Biosciences (GZMB)

Georg-August University, Goettingen

\section{DATE OF ORAL EXAMINATION:}


Affidavit:

I herewith declare, that this thesis has been written independently and with no other sources and aids than explicitly quoted.

I would like to use the term 'We' instead of 'I', because during my PhD thesis I was supported by other persons, in particular my supervisor and my lab members. However, the presented work in this dissertation and the writing were essentially done and evaluated by myself.

Sarah Wälde 



\section{TO SCIENCE}





\section{Content}

$\begin{array}{ll}\text { ABSTRACT } & 1\end{array}$

1 INTRODUCTION 3

1.1 The basic mechanisms of nucleocytoplasmic transport 3

1.1.1 Importins and exportins mediate nuclear transport 3

1.1.2 Import receptors bind to distinct nuclear localization signals (NLS) 5

$\begin{array}{lll}\text { 1.1.3 The RanGTP-gradient in interphase } & 6\end{array}$

1.1.4 The transport cycle of the importin $\alpha / \beta$ complex $\quad 7$

1.1.5 Nuclear export 9

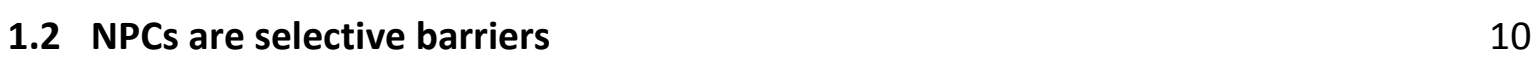

1.2.1 FG-Nups contribute to the permeability barrier of the NPC 12

1.2.2 The NPC permeability barrier 12

1.3 Certain FG-Nups are involved in nuclear import $\quad 15$

$\begin{array}{ll}\text { 1.3.1 Nup358 is a multi-domain protein } & 16\end{array}$

1.3.2 Depletion of Nup358 leads to reduced nuclear import of reporter proteins 18

1.3.3 Nuclear import of a subset of proteins depends on Nup358 19

$\begin{array}{ll}1.4 \text { Aim of the work } & 21\end{array}$

2 MATERIAL \& METHODS 23

2.1 Material 23

2.1.1 Chemicals, reagents and enzymes 23

2.1.2 Consumsables 25

2.1.3 Kits 25

2.1.4 Buffers, stock solutions and media 26

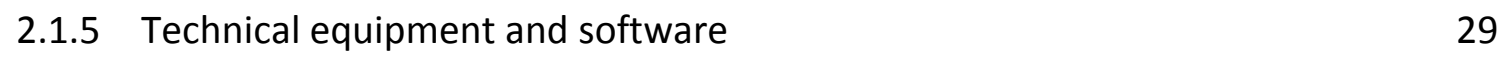

$\begin{array}{lll}2.1 .6 & \text { Cell lines } & 31\end{array}$

$\begin{array}{lll}2.1 .7 & \text { SiRNA } & 31\end{array}$

$\begin{array}{lll}2.1 .8 & \text { Oligonucleotides } & 32\end{array}$

$\begin{array}{ll}2.1 .9 & \text { Vectors and plasmids } \\ 2.1 .10 & 35\end{array}$

2.1.10 Antibodies $\quad 43$

2.1.11 Proteins 45

2.2 Molecular biology methods 46

2.2.1 Plasmid DNA purification $\quad 46$

2.2.2 Measurement of DNA concentration and purity 46

$\begin{array}{lll}\text { 2.2.3 } & \text { Polymerase chain reaction (PCR) } & 47\end{array}$

2.2.4 Agarose gel electrophoresis 48

2.2.5 Restriction of DNA by endonucleases 48 
2.2.6 Ligation of DNA fragments 49

2.2.7 Oligonucleotide cloning $\quad 49$

2.2.8 Sequencing of DNA 50

2.2.9 Site-directed mutagenesis $\quad 51$

2.2.10 Prokaryotic Cells $\quad 52$

2.3 Biochemical methods 53

2.3.1 Separation and detection of proteins 53

2.3.2 Protein transfer via western blot and immunological detection of proteins 54

2.3.3 Expression and purification of recombinant GST-tagged proteins 55

2.3.4 In vitro binding assays with GST- and His-labeled proteins 56

2.4 Cell biology methods for mammalian cell culture 57

$\begin{array}{lll}\text { 2.4.1 Cultivation of adherent cells } & 57\end{array}$

2.4.2 RNA interference (RNAi) experiments $\quad 57$

2.4.3 Coating of cover slips with Poly-L-Lysine 58

2.4.4 Transient transfection of DNA 58

2.4.5 In vitro import assays in HeLa cells 59

2.4.6 The dexamethasone-inducible import system 60

$\begin{array}{lll}2.4 .7 & \text { Immunofluorescence } & 61\end{array}$

2.4.8 Fluorescence microscopy 62

2.4.9 Quantification of import inhibition in Nup358-depleted cells 63

2.4.10 Immunoprecipitations 64

3 RESULTS 67

3.1 Nup358 depletion affects nuclear import and not export of a transportin cargo 67

3.2 Nuclear accumulation of a subset of proteins depends on Nup358 68

3.2.1 Nuclear accumulation of DDX43 and DDX59 requires Nup358 69

DDX43 and DDX59 bind to distinct import factors 71

3.2.2 Depletion of Nup358 causes extranuclear aggregations of Sox $6 \quad 72$

Different import factors can mediate nuclear import of Sox $6 \quad 74$

The C-terminal half of Sox 6 contains several NLSs $\quad 76$

3.2.3 Nuclear localization of DBC-1 depends on Nup358 77

Nuclear import of Sirt1 does not require Nup358 80

The importin $\alpha / \beta$ complex mediates nuclear import of DBC-1 81

Importins are not the only rate-limiting factors in nuclear import of DBC-1 85

The coiled-coil domain of DBC-1 is required for Nup358-dependent

nuclear localization

89 
3.3 The role of Nup358 in nuclear import of certain substrates 92

3.3.1 Exogenous Nup358 localizes to the nuclear pore 92

3.3.2 Exogenous Nup358 can rescue the import defect of DBC-1 95

3.3.3 siRNA-resistant truncation mutants of Nup358 97

3.3.4 The N-terminal part of Nup358 mediates nuclear import of DBC-1 101

Incorporation into the nuclear pore is required to compensate

for the loss of endogenous Nup358 to import DBC-1 103

The FG-repeat at amino acid 1141 alone does not mediate nuclear

$\begin{array}{ll}\text { import of DBC-1 } & 105\end{array}$

3.3.5 Analysis of the interaction between Nup358 and DBC-1 107

Soluble fragments of Nup358 inhibit nuclear import of DBC-1 107

DBC-1 can interact with the N-terminal part of Nup358 109

3.3.6 Nup358 can rescue the import defect of other reporter proteins 111

The N-terminal part of Nup358 mediates nuclear uptake of NES-GFP ${ }_{2}$-CNLS 111

Additional regions of Nup358 are required for nuclear import of

a transportin cargo

115

A similar region of Nup358 as needed for the transportin reporter can

mediate nuclear import of the HIV-1 Rev protein

Different nuclear transport pathways require distinct domains

of Nup358 for their import

\section{DISCUSSION}

4.1 Nuclear accumulation of a subset of proteins depends on Nup358

4.1.1 Nup358 depletion affects nuclear import and not export of the reporter proteins

4.2 Distinct import pathways are affected by depletion of Nup358

4.2.1 DDX43 and DDX59 bind to different import receptors

4.2.2 Several NTRs can mediate nuclear uptake of Sox 6

4.2.3 The importin $\alpha / \beta$ complex mediates nuclear import of DBC-1

The coiled-coil region of DBC-1 is involved in the Nup358 dependency

\subsection{Full-length Nup358 and its truncations are a very powerful tool} to investigate the involvement in nuclear transport

4.3.1 Pore-associated RanGAP1 and the E3 ligase activity of Nup358 are not required for nuclear import

4.3.2 Distinct domains of Nup358 mediate nuclear import of different import pathways

The N-terminal part of Nup358 is sufficient for nuclear transport

of cargos using the importin $\alpha / \beta$ pathway

4.3.3 Import of transportin cargos cannot be mediated by the $\mathrm{N}$-terminal part of Nup358 
4.4 Interactions between cargo and the NPC can occur by different mechanisms

4.4.1 The N-terminal part of Nup358 functions as an assembly platform for transport complexes with importin $\alpha / \beta$

4.4.2 Receptor-independent interaction between DBC-1 and Nup358

4.4.3 Import cargo and NTR can both contribute to nuclear pore interaction

4.5 Outlook

REFERENCES

APPENDIX

ABBREVIATIONS

ACKNOWLEDGEMENTS 


\section{ABSTRACT}

Nucleocytoplasmic transport occurs through nuclear pore complexes (NPC). Some nucleoporins, components of the NPC, have been suggested to function as initial or terminal binding sites for nuclear transport cargos. In vertebrates, the nucleoporin Nup358/RanBP2 is a major component of the cytoplasmic filaments, and some of its characteristics, like FG-repeats, Ran-binding domains (RanBDs) and the associated RanGAP1, may be directly linked to nuclear transport. However, it had been shown that Nup358 is dispensable for transport of standard cargos into the nucleus.

Contrary to these data, a subset of cellular proteins that localize to the nucleus under control conditions accumulated in the cytoplasm upon depletion of Nup358, including the putative tumor suppressor DBC-1 (Hutten, 2007). We demonstrated that depletion of Nup358 by RNA interference (RNAi) leads to reduced import rates of proteins using the importin $\alpha / \beta$ (Hutten et al., 2008) or transportin pathway (Hutten et al., 2009). These data suggested that Nup358 serves as a general docking site for specific transport complexes.

To control the specificity of the observed effect upon Nup358 depletion, an RNAi-resistant mutant of full-length Nup358 was designed. Transfection of exogenous full-length Nup358 could compensate for the loss of endogenous Nup358. We used this tool to delete specific domains of Nup358 in order to identify the responsible regions for nuclear accumulation of Nup358-dependent substrates. Nuclear import does not require pore-associated RanGAP1, as a mutant lacking the RanGAP1 interaction site could compensate for the loss of endogenous Nup358. Clearly, the region, which is sufficient for nuclear import, differs between the importin $\alpha / \beta$ - and transportin-dependent pathway. An N-terminal fragment, which does not contain any RanBD, could promote nuclear import of DBC-1 and an NLS-reporter, two substrates that use the importin $\alpha / \beta$ pathway. Furthermore, import of DBC-1 could be inhibited by soluble Nup358 fragments spanning amino acids 1000-1306. Biochemically, we could verify an association between DBC-1 and the N-terminal part of Nup358 which was independent of nuclear transport receptors. 
By contrast, transportin-dependent cargos require a different region of Nup358 for nuclear import.

Altogether, these data suggest that the N-terminal third of Nup358 fulfills the function as an assembly/disassembly platform for cargos of the importin $\alpha / \beta$ pathway. Loss of Nup358-associated RanGAP1 and the four RanBDs of Nup358 can be overtaken by soluble proteins. Additional regions of Nup358 seem to be required for transportin-dependent import. 


\section{INTRODUCTION}

The accessibility of cytoplasmic proteins to the genetic material inside the nucleus is restricted by the physical barrier of the nuclear envelope (NE). Hence, eukaryotic cells have achieved a complexity in transcriptional regulation that is not found in prokaryotes. Furthermore, the NE provides additional levels of regulation of gene expression, such as the selective export of newly synthesized mRNA to the site of translation in the cytoplasm. On the other hand, this compartmentalization necessitates a whole orchestra of different proteins to facilitate the nucleocytoplasmic exchange of the molecules. How does a protein destined for the nucleus move from the cytoplasm into the nuclear interior? Clearly, the selection of substrates for transport serves as an important point for controlling cell functions although small molecules and proteins can enter the nucleus passively (reviewed in Fried et al., 2003; Mosammaparast et al., 2004; Pemberton et al., 2005).

\subsection{The basic mechanisms of nucleocytoplasmic transport}

\subsubsection{Importins and exportins mediate nuclear transport}

Selective transport between nucleus and cytoplasm in eukaryotes occurs through nuclear pore complexes (NPC), multi-protein complexes that consist of nucleoporins (Nups) and provide channels for transport across the NE (Goldberg et al., 1996). Soluble nuclear transport factors (NTRs), also named karyopherins, recognize a specific sequence within the cargos and facilitate their transport in a highly regulated manner (reviewed in Fried et al., 2003; Weis, 2003; Mosammaparast et al., 2004; Pemberton et al., 2005; Stewart, 2007).

The importin $\beta$-like family of NTRs represents the largest class of NTRs (reviewed in Fried et al., 2003; Pemberton et al., 2005) with import carriers called importins 
(Görlich et al., 1994) and export carriers called exportins (Stade et al., 1997). Features of this protein family are a similar overall structural organization, an acidic isoelectric point and a molecular weight between 90-145 kDa. They consist of several HEAT repeats and share a low sequence similarity, which is $<20 \%$ and mostly restricted to the $\mathrm{N}$-terminus (reviewed in Chook et al., 2001; Mosammaparast et al., 2004). The HEAT repeat is a helix-loop structure named after proteins where it was first identified (Huntingtin, elongation factor 3 (ㅌF3), protein phosphatase 2A (PP2A)), and the yeast PI3 kinase IOR1 (Andrade et al., 1995; Andrade et al., 2001). Normally, NTRs can bind the small GTP-hydrolase (GTPase) Ran loaded with GTP through their N-terminus (Chi et al., 1996; Kutay et al., 1997b; Chook et al., 1999). Further, they contain one or multiple binding sites for certain Nups, the phenylalanine-glycine (FG-) Nups (Radu et al., 1995; Rexach et al., 1995) (see chapter 1.2.1). Based on these features, more than 20 members of the importin $\beta$ family have been identified so far in metazoans (reviewed in Macara, 2001; Fried et al., 2003; Mosammaparast et al., 2004).

Nuclear import of specific cargos can be mediated for example by importin $\beta$ (Görlich et al., 1995a; Chi et al., 1995), transportin 1 (Pollard et al., 1996), transportin SR1 (Kataoka et al., 1999) and SR2 (Lai et al., 2001), importin 4 (Jäkel et al., 2002), importin 5 (Yaseen et al., 1997; Deane et al., 1997; Jäkel et al., 1998), importin 7 (Jäkel et al., 1998; Jäkel et al., 1999), importin 8 (Dean et al., 2001), importin 9 (Mühlhäusser et al., 2001; Jäkel et al., 2002) and importin 11 (Plafker et al., 2000). Exportin 1 (CRM1) (Fornerod et al., 1997a), CAS (Kutay et al., 1997a), exportin t (Arts et al., 1998; Kutay et al., 1998), exportin 5 (Calado et al., 2002) and exportin 6 (Stüven et al., 2003) facilitate nuclear export. Some NTRs can function as importins and exportins, like importin 13 (Mingot et al., 2001) and exportin 4 (Lipowsky et al., 2000; Gontan et al., 2009).

Several distinct receptors can mediate nuclear uptake of the same cargo (reviewed in Mosammaparast et al., 2004; Pemberton et al., 2005; Tran et al., 2007). For example, core histones can be transported by importin $\beta$, transportin, importin 5 , importin 7 as well as importin 9 (Baake et al., 2001; Mühlhäusser et al., 2001). Hence, it seems that proteins with important functions in the nucleus take redundant pathways to get access to this organelle. 


\subsubsection{Import receptors bind to distinct nuclear localization signals (NLS)}

The first step of nuclear import marks the specific binding of an import receptor to a transport cargo. The interaction sites within the cargos are thought to be small signal sequences called nuclear localization signals (NLS) (reviewed in Fried et al., 2003; Stewart, 2007).

The most prominent transport signal is the classical NLS (cNLS), containing either one (monopartite) or two (bipartite) clusters of basic residues (Dingwall et al., 1982; Kalderon et al., 1984a; Robbins et al., 1988). The monopartite NLS contains a single cluster of 4-5 basic residues as exemplified by the Simian virus 40 (SV40) large T antigen with the sequence PKKKRKV (basic residues are underlined) (Kalderon et al., 1984b; Feldherr et al., 1994). The best studied bipartite cNLS is found in the Xenopus laevis protein nucleoplasmin, which has a second basic cluster located downstream of the first KRPAATKKAGQAKKK (Dingwall et al., 1982; Robbins et al., 1988). However, length of the linker sequence can vary enormously with up to 25 amino acids in Rrp4, a subunit of the exosome which expands the bipartite consensus beyond the previous limitations of 9-12 residues (Lange et al., 2010). By structural and thermodynamical studies, the residues $K(K / R) X(K / R)$ have been identified as key requirements for a $c N L S$ (Conti et al., 2000; Fontes et al., 2000; Hodel et al., 2001).

Cargo proteins with such a classical NLS are transported mostly by the importin $\alpha / \beta$ dimer. Importin $\alpha$, an adaptor protein that is not a member of the family of importin $\beta$ related NTRs, is a $\sim 60 \mathrm{kDa}$ protein responsible for recognizing and binding of the NLS in import substrates (Weis et al., 1996; Görlich et al., 1996a). Importin $\beta$ binds the adaptor importin $\alpha$ and mediates translocation through the NPC (Görlich et al., 1995b).

The association site within the $\mathrm{N}$-terminus of importin $\alpha$ to importin $\beta$ is called importin $\beta$-binding (IBB-) domain (Görlich et al., 1995a), and contains a cluster of basic residues (KRR) similar to a NLS (Conti et al., 1998; Fanara et al., 2000). When the IBB does not interact with importin $\beta$, it competes with a NLS-containing cargo for binding to importin $\alpha$. This autoinhibitory mechanism is important for cargo release in the nucleus after translocation through the pore (Kobe, 1999; Harreman et al., 2003a; Harreman et al., 2003b). 
Besides importin $\alpha$, importin $\beta$ can also use other NTRs as adaptors to mediate nuclear import of certain substrates, for example importin 7 for histone H1 (Görlich et al., 1997; Jäkel et al., 1999). Additionally, there are also cargos transported by importin $\beta$ alone like the T-cell protein tyrosine phosphatase (Tiganis et al., 1997) or cyclin B1 (Moore et al., 1999), but it often binds to a non-classical NLS like the parathyroid hormone-related protein (PTHrP) (Cingolani et al., 2002). Hence, a general NLS-prediction is difficult because most of the substrates imported by importin $\beta$ alone share little sequence or structural homology (Cingolani et al., 2002; Lee et al., 2003).

Besides the importin $\alpha / \beta$ pathway, transportin is another important NTR that binds the cargos directly. Transportin recognizes its most prominent substrate, hnRNP A1, through the M9-NLS, a 38 residues stretch, which is enriched in glycine residues and deficient in basic amino acids (Siomi et al., 1995; Pollard et al., 1996; Bonifaci et al., 1997). Further, crystal structure analysis has identified a proline-tyrosine (PY-) NLS as a new binding motif for transportin (Lee et al., 2006), which is characterized by structurally disordered sequence and by an overall positive charge followed by a C-terminal R/K/HX $\mathrm{H}_{(2-5)} / \mathrm{PY}$-motif.

\subsubsection{The RanGTP-gradient in interphase}

The small GTPase Ran regulates the directionality of nuclear transport (reviewed in Pemberton et al., 2005; Stewart, 2007). Ran belongs to the evolutionary highly conserved branch of the Ras superfamily that is found in the nucleus and cytoplasm (Drivas et al., 1990; Bischoff et al., 1991a, b). Like other GTPases, Ran cycles between a GTP- and a GDP-bound form, which are asymmetrically distributed between nucleus and cytoplasm, respectively (Mattaj et al., 1998; Görlich et al., 1999; Lei et al., 2002). Nucleotide loading of Ran is controlled and catalyzed by the chromatin-bound nucleotide exchange factor RCC1 (RanGEF) in the nucleus (Bischoff et al., 1991a) and the cytoplasmic GTPase-activating protein RanGAP1 (Bischoff et al., 1994; Klebe et al., 1995), which is associated with the cytoplasmic side of the NPC (Matunis et al., 1996; Mahajan et al., 1997). 
RanGTP in a stable complex with any NTR translocates through the pore to the cytoplasm (Bischoff et al., 1997; Mattaj et al., 1998; Görlich et al., 1999; Macara, 2001). As this complex is resistant to RanGAP1 induced GTP-hydrolysis (Floer et al., 1996), its dissociation is facilitated by another family of Ran-binding proteins, RanBP1 (Bischoff et al., 1995) and RanBP2 (Nup358) (Yokoyama et al., 1995; Wu et al., 1995). Hence, interaction with the Ran-binding domains (RanBD) of RanBP1 or Nup358 has been shown to weaken the RanGTP/NTR complex, and allows RanGAP1 to stimulate Ran's GTPase activity (Bischoff et al., 2002).

The asymmetrical distribution of RanGTP regulates the direction of cargo movement through the central channel of the NPC (reviewed in Fried et al., 2003). Even though for every transport cycle, at least one molecule RanGTP is depleted from the nucleus, its steady-state localization is nuclear. This is achieved very efficiently by import of RanGDP via the RanGDP-specific import receptor NTF2 (nuclear transport factor 2) (Paschal et al., 1995; Clarkson et al., 1996; Ribbeck et al., 1998; Smith et al., 1998). This process is very efficient and rapid with several million molecules of Ran transported into the nucleus per minute (Mattaj et al., 1998). After entering the nucleus, RCC1 triggers disassembly of the RanGDP/NTF2 complex by promoting nucleotide exchange on Ran into its GTP-bound form (Figure 1-1 A) (Ribbeck et al., 1998; Smith et al., 1998).

\subsubsection{The transport cycle of the importin $\alpha / \beta$ complex}

In the cytoplasm, cargo proteins containing a cNLS are recognized by the adaptor receptor importin $\alpha$, which in turn binds to importin $\beta$ through its IBB-domain (Görlich et al., 1995b; Görlich et al., 1995a). This import complex facilitates movement through the NPCs (Figure 1-1 B) (see also chapter 1.2).

In the nucleus, binding of RanGTP to importin $\beta$ causes a conformational change of the NTR leading to disassembly into RanGTP/importin $\beta$ and importin $\alpha /$ cargo by an allosteric mechanism (Figure 1-1 C) (Vetter et al., 1999; Cingolani et al., 1999; Chook et al., 2002). 

A
B
C

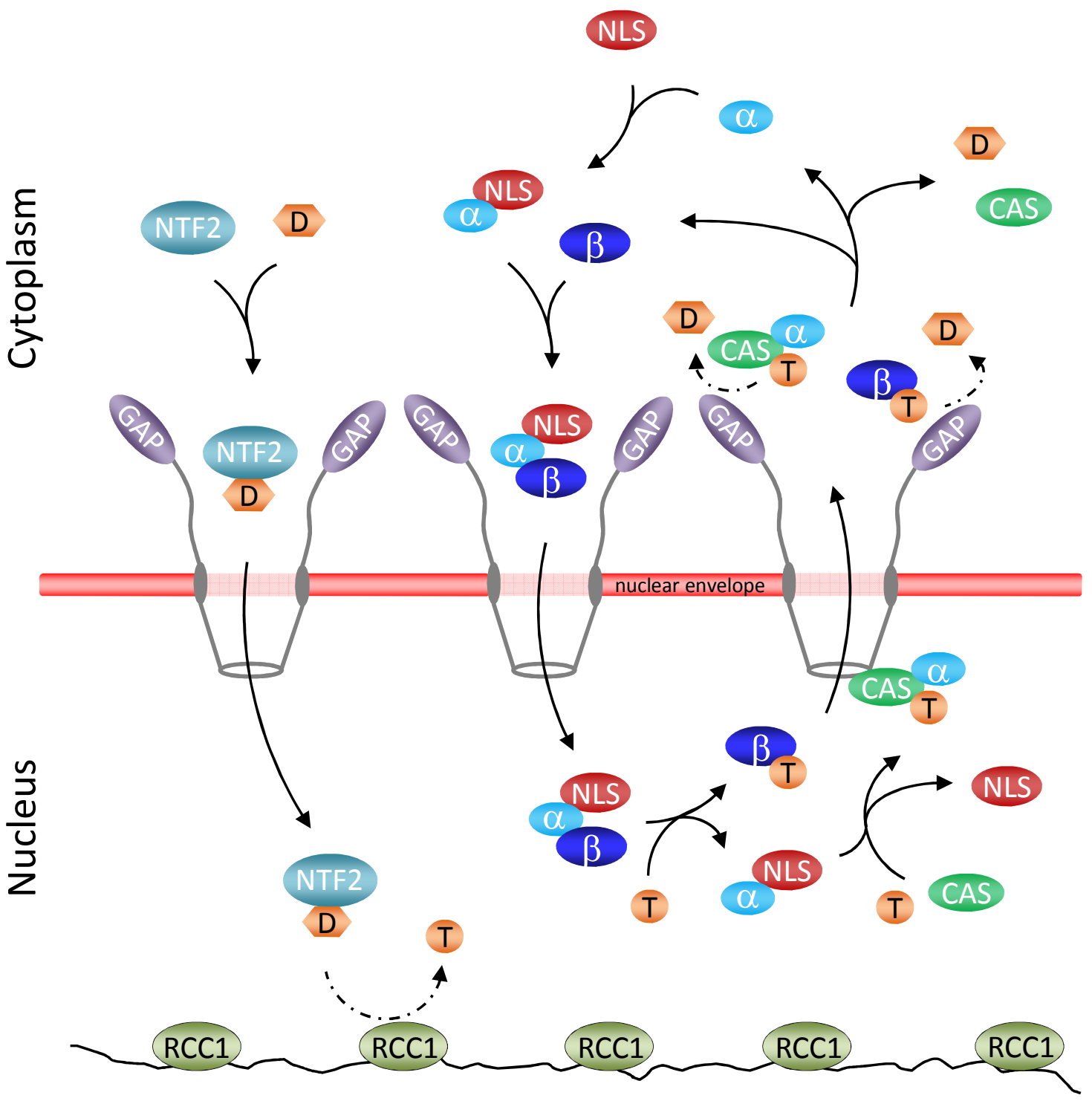

Figure 1-1: Model of the importin $\alpha / \beta$ transport cycle

A: NTF2 imports the GTPase Ran loaded with GDP (D). RCC1 triggers disassembly of the RanGDP/NTF2 complex by promoting nucleotide exchange on Ran into its GTP-bound form (T). B: Nuclear import of an NLS-containing cargo (NLS) uses the importin $\alpha / \beta$ pathway. C: The importin $\beta /$ RanGTP and CAS/importin $\alpha /$ RanGTP complexes are recycled to the cytoplasm. RanGAP1 controls and catalyzes the hydrolysis of RanGTP to RanGDP which leads to disassembly of the complex. RanGAP1 (GAP) is anchored to the cytoplasmic face of the NPC, and RCC1 is bound to chromatin. T: RanGTP; D: RanGDP; $\alpha$ : importin $\alpha$; $\beta$ : importin $\beta$, NLS: NLS-containing import cargo. For simplicity, accessory factors are left out. See text for more details. 
To release the cargo from importin $\alpha$, the IBB-domain decreases the affinity of the cargo to importin $\alpha$ through an autoinhibitory mechanism (Conti et al., 1998; Kobe, 1999). However, importin $\alpha$ has a relatively high affinity for the NLS, and this self-induced release cannot accommodate for the high transport rates of the NPC that has been estimated to $100-1,000$ cargos per minute per NPC (Ribbeck et al., 2001; Timney et al., 2006). Hence, Nup50, a nucleoporin at the nuclear side of the NPC, offers binding sites for the importin $\alpha /$ cargo complex, and also recruits RanGTP and CAS (cellular apoptosis susceptibility), the export factor for importin $\alpha$ (Kutay et al., 1997a). Presence of these factors accelerates importin $\alpha /$ cargo disassembly (Matsuura et al., 2003). Furthermore, Nup50 actively displaces the NLS-cargo from importin $\alpha$ (Gilchrist et al., 2002; Matsuura et al., 2005), and CAS binds predominantly non-loaded importin $\alpha$, which supports recycling of the transport receptor without cargo (Kutay et al., 1997a).

After disassembly of the import complex, importin $\beta /$ RanGTP is recycled through the NPC to the cytoplasm (Görlich et al., 1999; Macara, 2001; Chook et al., 2001; Pemberton et al., 2005). Export of importin $\alpha$ in complex with RanGTP is mediated actively by CAS (Matsuura et al., 2004). In the cytoplasm, hydrolysis of RanGTP to RanGDP by RanGAP1 and the RanBDs of RanBP1 and Nup358 disassembles these complexes, and the NTRs are empty for another import round (reviewed in Pemberton et al., 2005; Stewart, 2007).

\subsubsection{Nuclear export}

Exportins facilitate nuclear export of a multitude of different proteins, RNA molecules and ribonucleoprotein particles (RNPs) and bind to specific signals within the cargo sequence, referred to as nuclear export signals (NES) (reviewed in Fried et al., 2003; Weis, 2003). CRM1 (chromosome region maintenance 1; also known as exportin 1 or Xpo1) is the best characterized of all export factors, and is involved in export of proteins such as cell cycle regulators, transcription factors, RNA binding proteins and numerous others (Fornerod et al., 1997a; Stade et al., 1997). It recognizes a hydrophobic NES that is characterized by a leucine-rich sequence originally identified in the HIV-1 Rev protein and PKI (Fischer 
et al., 1995; Wen et al., 1995). The function of CRM1 can be inhibited efficiently and specifically by the antifungal agent leptomycin B (LMB) (Kudo et al., 1998), which reacts irreversibly with a cysteine residue within the NES-binding domain (amino acid 529 in human CRM1) (Kudo et al., 1999).

The export complex consists of CRM1, RanGTP and the NES-cargo (Fried et al., 2003). Formation of the trimeric export complex in the nucleus is assisted by the soluble factor RanBP3 (Englmeier et al., 2001; Lindsay et al., 2001; Nemergut et al., 2002). The complex then translocates through the NPC and interacts with the Nup214/Nup88 complex on the cytoplasmic side of the NPC (Fornerod et al., 1997b; Kehlenbach et al., 1999; Hutten et al., 2006). After translocation through the NPC, the cytoplasmic proteins RanBP1, Nup358, RanGAP1 and NXT1 (Black et al., 1999; Black et al., 2001), a protein also involved in mRNA export, facilitate disassembly of the complex, and RanGTP can be hydrolyzed to RanGDP (Kehlenbach et al., 1999; Englmeier et al., 1999, also reviewed in Macara, 2001; Fried et al., 2003).

\subsection{NPCs are selective barriers}

Nucleocytoplasmic transport occurs through nuclear pore complexes (NPCs), large channels that span the inner and the outer nuclear membranes (reviewed in Fried et al., 2003; Stewart, 2007). NPCs selectively regulate transport of macromolecules larger than $40 \mathrm{kDa}$, but ions, small metabolites and small proteins can pass by free diffusion (Paine et al., 1975, also reviewed in Fried et al., 2003; Weis, 2003). Yeast and vertebrate NPCs are structurally similar and consist of multiple copies of $\sim 30$ different nucleoporins (Nups) (Rout et al., 2000; Cronshaw et al., 2002; Rout et al., 2003), which localize on both sides of a symmetry axis in the plane of the NE (reviewed in D'Angelo et al., 2008). Exceptions are some peripheral, asymmetric nucleoporins like the cytoplasmic Nup358 (Wu et al., 1995; Yokoyama et al., 1995; Walther et al., 2002), Nup214 (Kraemer et al., 1994) and the nuclear Nup153 (Sukegawa et al., 1993). The assembly of several copies of each Nup, multiples of eight, reflect the apparent eightfold symmetry of the NPC resulting in a high molecular mass complex of $60-125 \mathrm{mDa}$ in mammals and 
40-60 mDa in yeast with 500-1,000 Nups per pore (Akey et al., 1993; Rout et al., 2000; Alber et al., 2007b).

The structure includes a NE-embedded scaffold that surrounds a central transport channel, referred to as the spoke complex or spoke ring, which is sandwiched by two rings (Goldberg et al., 1996). Eight filaments are attached to each of these rings, the cytoplasmic ones emanating with loose ends into the cytoplasm whereas the nuclear filaments are linked to a distal ring, forming the basket-like structure (Figure 1-2) (reviewed in Terry et al., 2009).

The NPC-proteome includes three pore membrane nucleoporins (POMs), for anchoring the complex into the NE. The scaffold is formed by two main protein subcomplexes linking a set of phenylalanine-glycine (FG-) repeats containing Nups to the Poms (Alber et al., 2007b; Alber et al., 2007a).

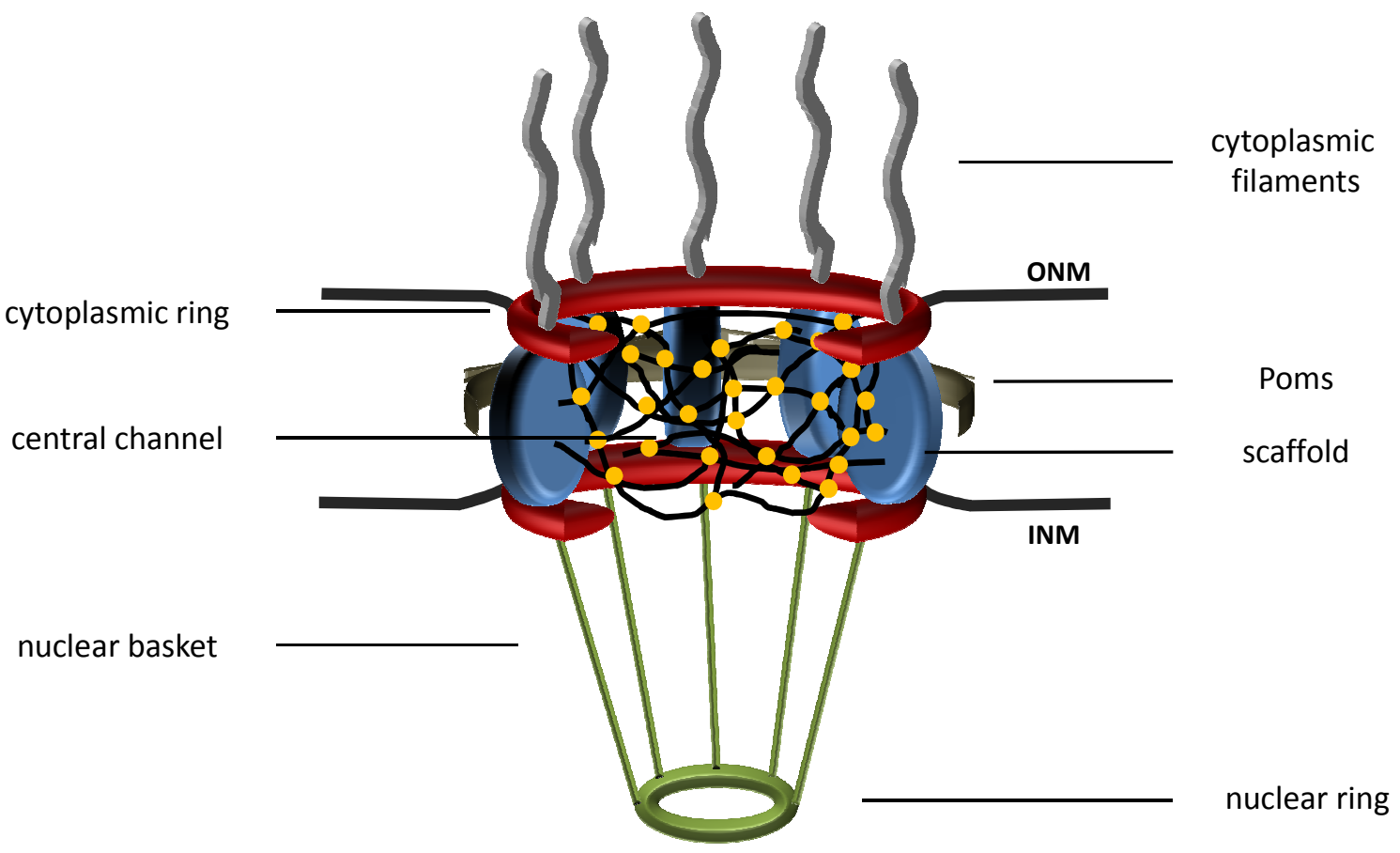

Figure 1-2: Structure and composition of the nuclear pore complex (NPC) (Wälde et al., 2010)

A model of the vertebrate NPC with only five out of eight filaments is shown. The permeability barrier in the central channel is illustrated as a meshwork of FG-Nups, yellow dots indicate FG-FG contacts. ONM and INM: outer and inner nuclear membrane. 


\subsubsection{FG-Nups contribute to the permeability barrier of the NPC}

FG-Nups represent a third of the total Nups, filling up the channel of the NPC and extending into the cytoplasmic and nucleoplasmic volume. There are 3,500 FG-repeats per NPC (Rout et al., 2000; Cronshaw et al., 2002). The core unit of each FG-repeat is Phe-Gly (FG), Gly-Leu-Phe-Gly (GLFG) or Phe-any-Phe-Gly (FXFG) containing up to 48 motifs per repeat (Wente et al., 1994).

FG-repeats can interact with transport receptors (Damelin et al., 2000; Denning et al., 2003; Strawn et al., 2004), and binding can be completely abolished by the exchange of phenylalanine to alanine in the repeats (Patel et al., 2008). Different transport receptors prefer binding to distinct FG-domains (Marelli et al., 1998; Damelin et al., 2000). As there are multiple NTR binding sites within one FG-domain, up to 48 FG-domains within one FG-Nup and multiple FG-binding pockets on the surface of the NTRs (Rexach et al., 1995; Radu et al., 1995; Shah et al., 1998a), the avidity of binding sites may contribute to transport of molecules (Pemberton et al., 2005). These interactions are rather weak and likely transient, suggesting multiple low binding events to ensure proper and efficient transport (Rout et al., 2003; Pyhtila et al., 2003).

\subsubsection{The NPC permeability barrier}

Each of the $\sim 2,800$ NPCs in one HeLa cell can transport up to 60,000 molecules per minute (Feldherr et al., 1984; Görlich et al., 1996b). This results in 1,000 translocations events per second, shuttling a mass of $\sim 100 \mathrm{mDa}$ (Ribbeck et al., 2001), and demonstrates a very robust, efficient and bidirectional transport process. Hence, passage of transport complexes is not significantly decreased compared to smaller molecules passing by free diffusion (Gilchrist et al., 2002; Fried et al., 2003).

Mostly, FG-Nups are located at peripheral, surface accessible positions, by this lining the innermost layer of the central channel (Alber et al., 2007b). They participate in the permeability barrier of the NPC as pores depleted of specific FG-domains are 'leaky' for the diffusion of inappropriate molecules (Patel et al., 2007). However, a systematic 
deletion of the FG-repeats in 11 different Nups in yeast revealed that the FG-domains of asymmetrically located Nups were not essential for nuclear import of an NLS-cargo (Strawn et al., 2004).

So far, several models have been suggested to explain the selectivity of the NPC taking into account that active transport through the pore occurs by facilitated diffusion and requires the interaction of NTRs with the FG-nucleoporins.

In the 'selective phase model/hydrogel model' (Figure 1-3 A), the FG-Nups from the central channel form a sieve-like network through weak hydrophobic interactions between the FG-repeats (Ribbeck et al., 2001). This gel-like meshwork enables the passage of small molecules, but particles larger than the pore size are excluded. NTRs help transport cargos to translocate through the pore by increasing their solubility in the central channel by interacting with the FG-nucleoporins. Recently, it has been demonstrated that recombinant FG-Nups can form a hydrogel in vitro under certain conditions (Frey et al., 2006; Frey et al., 2007).

The 'virtual gate model' (Figure 1-3 B) proposes the existence of an energetic barrier controlling NPC-permeability. Here, highly dense, non-cohesive FG-Nups of the central channel generate an entropic barrier preventing the passage of inert molecules (Rout et al., 2003). A diffusing molecule in the cytoplasm enters the central channel of the pore leading to an immediate restriction of its liberty of movement and a drop of its entropy. Interaction of a molecule with the FG-Nups increases the probability of entering and moving through the pore.

A rather new model is the 'forest model' (Figure 1-3 C) that combines the features of the selective phase and virtual gate model. Rexach and coworkers suggest that FG-domains are structurally and chemically heterogeneous (Yamada et al., 2010). They postulate a forest-like landscape with 'shrubs' and 'trees' reaching into the volume of the NPC. Shrubs largely adopt a globular, collapsed conformation with low charge content, thereby positioning the FG-domain in close proximity to an anchor domain that links the FG-Nup close to the wall of the NPC. Trees contain a globular, collapsed-coil domain that is separated from the anchor domain by an FG-domain in a more dynamic, extended-coil conformation. Hence, a tubular gate structure or transporter at the NPC center exists allowing transport as described in the selective phase model. In contrast to the selective 
phase model, here two separate zones for traffic surround the transporter. For these zones, the extended or relaxed coil regions might function like an entropic gate as described for the 'virtual gate model'.

In the 'reduction of dimensionality model' (Figure 1-3 D), the binding sites for NTRs on FG-Nups are always saturated with NTRs leading to a permanent collapse of the FG-Nups (Peters, 2009).

A Selective phase/hydrogel model

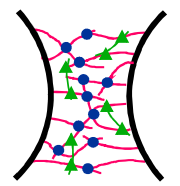

C Forest model

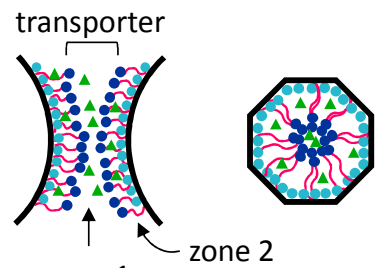

B Virtual gate model
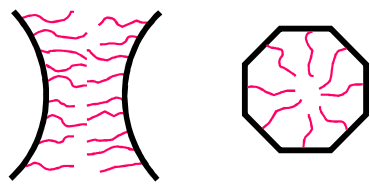

D Reduction of dimensionality model

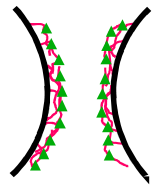

Figure 1-3: Nuclear transport models (Wälde et al., 2010)

A: In the selective phase model, FG-nucleoporins (pink) form a sieve-like meshwork with the function of a permeability barrier. The pores of the sieve are formed by FG-FG contacts (blue dots) and are tightened by additional binding of NTRs (green triangles) to the FG-domains. As NTRs have multiple FG-binding sites, they contribute to the permeability barrier (Ribbeck et al., 2001; Frey et al., 2006; Frey et al., 2007). B: In the virtual gate model, largely non-cohesive FG-Nups arrange as 'polymer brushes' (pink) forming an entropic barrier. Consequently, translocation of large molecules is energetically unfavorable. However, NTRs by binding to FG-domains can enter the transport channel (Rout et al., 2003). C: The forest model describes FG-domains with a globular, collapsed conformation (shrubs, light blue dots) and/or extended-coil conformation (trees) contributing to a forest-like landscape. Transport zone 1 in the interior of the NPC is built of the globular domains of trees (dark blue dots), and the peripheral zone 2 contains the extended-coil regions of trees (pink lines). NTRs (green) can pass through zone 1 or 2 regarding the size of the transport complex (Yamada et al., 2010). D: The reduction of dimensionality model has the wall of the transport channel coated with NTRs which bind to FG-nucleoporins. Small molecules can diffuse passively through this tube. Transport complexes move by a random, two-dimensional walk on the inner wall of the channel (Peters, 2009).

(A-D), side views (left) and top views (right) of the NPC 
Hence, a central channel exists where small molecules can diffuse through, but larger molecules would be hindered. Transport receptors bind to the FG-surface in the cytoplasmic or nucleoplasmic side of the NPC and move through the pore by a two-dimensional walk, eliminating empty receptors from their binding sites. However, it is not clear, whether binding of transport receptors to FG-Nups results to the postulated collapse, as the observations here are controversy (Lim et al., 2007; Eisele et al., 2010). In order to investigate these models further, it is important to describe the biophysics of the FG-Nups and their interaction within the tiny volume of a 'real' NPC.

\subsection{Certain FG-Nups are involved in nuclear import}

Regardless of the transport model, particular FG-Nups can impair specific, single transport pathways, either import or export. Thus, nucleocytoplasmic transport of specific cargos can be regulated at the level of the nucleoporins.

For example, depletion of the p62/p58/p54 subcomplex leads to impaired import of a NLS-reporter cargo in insect cells (Sabri et al., 2007). A number of other nucleoporins are involved in nuclear import like the NDC-1-dependent transport of the SV40-NLS as shown in HeLa cells (Yamazumi et al., 2009). Tpr, which is located at the nuclear basket of the NPC and doesn not contain FG-repeat, is required for nuclear import of cNLS- and M9-cargos in mice whereas import of the ribosomal protein L23a is not affected (Wu et al., 2001).

Some Nups function especially in termination of nuclear import like Nup50 that displaces cNLS-cargos from the importin $\alpha / \beta$ complex through binding to importin $\alpha$ (Matsuura et al., 2005). Further, Nup153 was suggested as a termination platform for protein import as it has the highest affinity for importin $\beta$ in the hierarchy of Nups in the channel of the NPC in Xenopus extracts (Shah et al., 1998b).

Initiation of nuclear import might be linked to the cytoplasmic Nup358 as the filamentous protein reaches into the cytoplasm (Wu et al., 1995; Yokoyama et al., 1995; Delphin et al., 1997; Walther et al., 2002). Indeed, it was proposed that GTP-hydrolysis of Ran is an early step in nuclear import and occurs at Nup358 (Melchior et al., 1995a). 


\subsubsection{Nup358 is a multi-domain protein}

The FG-Nup Nup358/RanBP2 is the largest nucleoporin and with a length of $36 \mathrm{~nm}$ the major component of the cytoplasmic filaments (Figure 1-2) (Wu et al., 1995; Yokoyama et al., 1995; Delphin et al., 1997; Walther et al., 2002). Together with Nup214 and Nup88, they build the cytoplasmic face of the NPC (reviewed in D'Angelo et al., 2008). However, Nup358 is not part of the cytoplasmic subcomplex of Nup214/Nup88 as depletion of Nup358 did not reduce the levels of Nup214 or Nup88 and vice versa (Walther et al., 2002; Hutten et al., 2006). As a giant protein (358 kDa) with multi-domain architecture, Nup358 interacts with many different proteins as shown in Figure 1-4.

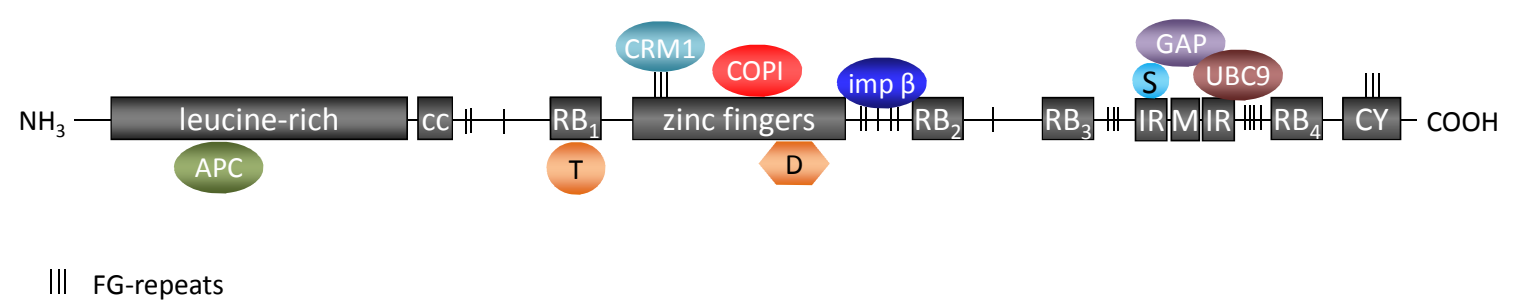

Figure 1-4: The cytoplasmic Nup358 is a multi-domain protein.

A schematic description of Nup358 is shown here with some known interaction partners like the APC-protein (Joseph et al., 2008; Murawala et al., 2009), CRM1 (Singh et al., 1999), COPI (Prunuske et al., 2006), RanGTP (T) and RanGDP (D) (Yaseen et al., 1999), SUMO-modified (S) RanGAP1 (GAP) and Ubc9 (Saitoh et al., 1996; Mahajan et al., 1997).

The eight zinc fingers in the middle part of Nup358 interact with different proteins providing several regulatory mechanisms. Hence, the protein helps to recruit the coatomer complex COPI, which is an important player in nuclear envelope breakdown, to the nuclear pore (Liu et al., 2003; Prunuske et al., 2006). Further, it shows zinc-dependent association with the nuclear transport machinery by interaction with the empty export factor CRM1 (Singh et al., 1999) or with the small GTPase RanGDP, suggesting an increased local concentration of RanGDP at the nuclear pore (Yaseen et al., 1999). Moreover, RanGDP (Yaseen et al., 1999) as well as RanGTP bind to the four RanBDs of 
Nup358 which are homologous to the Ran-binding domains of RanBP1 (Coutavas et al., 1993; Yokoyama et al., 1995; Wu et al., 1995; Melchior et al., 1995a).

Besides Ran, also the Ran-activating protein RanGAP1 associates with a region located in the C-terminal part of Nup358 through its SUMO1-modification (small ubiquitin-like modifier) (Mahajan et al., 1997; Matunis et al., 1998). Further, Ubc9, the single E2 enzyme for sumoylation of proteins, is part of this stable complex (Saitoh et al., 1996; Lee et al., 1998). Interestingly, Nup358 itself acts as SUMO E3 ligase with the catalytical domain located between RanBD3 and RanBD4. Possibly, Nup358 modifies many targets on their way into the nucleus suggesting a broad role for SUMO in regulation of nuclear trafficking (Pichler et al., 2002).

Nup358 also contains several FG-repeats, which may interact with various transport receptors like importin $\beta$ (Delphin et al., 1997).

\subsubsection{The Nup358/RanGAP1 complex associates to kinetochores during mitosis}

The association between RanGAP1/Nup358 is stable throughout the entire cell cycle (Mahajan et al., 1997; Matunis et al., 1998). During disassembly of the NPC in mitosis (reviewed in D'Angelo et al., 2008), RanGAP1/Nup358 are targeted to kinetochores by spindle microtubule attachment (Joseph et al., 2002) and recruits other proteins like the checkpoint proteins Mad1 and Mad2 (Salina et al., 2003). Hence, the RanGAP1/Nup358 complex operates kinetochore formation and maturation as spindle assembly and function are disturbed in the absence of Nup358 (Joseph et al., 2002; Salina et al., 2003; Joseph et al., 2004). Interestingly, a pool of cytoplasmic Nup358 is enriched at cell extensions to associate with interphase microtubules modulating their stability (Joseph et al., 2008). Furthermore, it has been suggested that Nup358 functions in cell polarity by regulating the localization of APC, a protein involved in microtubule function and polarization (Murawala et al., 2009).

In addition, sumoylation of Topo II $\alpha$ isomerase by Nup358 regulates the localization of the protein to the inner centrosomes to separate chromosomes prior to anaphase (Dawlaty et al., 2008). Nup358 $\%$ mice are embryonic lethal (Aslanukov et al., 2006). Hence, Nup358 hypomorphic mice (Nup358 ${ }^{+-}$), which have reduced levels of the protein, 
fail to accumulate the enzyme at the centrosomes. These animals develop certain tumors, suggesting a new role for the nucleoporin in suppression of tumorigenesis (Dawlaty et al., 2008). In this context, a critical role in metabolic processes and diseases can be referred to the leucine-rich domain at the very $\mathrm{N}$-terminus which exhibits chaperone activity towards two mitochondrial proteins, Cox11 and hexokinase type I (HKI) (Aslanukov et al., 2006). The partial loss-of-function in Nup358 ${ }^{+/-}$mice decreases growth, downregulates HKI and ATP levels selectively in the central nervous system, and causes visual problems.

\subsubsection{Depletion of Nup358 leads to reduced nuclear import of reporter proteins}

Some of the above mentioned characteristics may be directly linked to nuclear transport. Indeed, early studies on the mechanisms of signal-mediated nuclear import of proteins have revealed that import substrates bearing NLSs initially docked at the cytoplasmic filaments before translocation through the nuclear pore (Richardson et al., 1988; Pante et al., 1993). So Nup358 might act as a platform for the assembly/disassembly of transport complexes before or after translocation through the NPC.

It therefore came as a surprise that Nup358 is apparently dispensable in nucleocytoplasmic transport of proteins. For example, nuclear uptake of certain substrates was not impaired in cells depleted of Nup358 like a NLS- or M9-cargo in Xenopus oocytes (Walther et al., 2002), the glucocorticoid receptor (Salina et al., 2003) or the transcription factor NFAT in HeLa cells (Hutten et al., 2006).

In contrast some proteins like the PYM protein displayed Nup358-dependent nuclear import in Drosophila cells (Forler et al., 2004; Sabri et al., 2007). In this regard, nuclear uptake of the artificial reporters using the importin $\alpha / \beta$ or transportin pathway are significantly slowed down in cells depleted of Nup358 via RNA interference (Hutten et al., 2008; Hutten et al., 2009). Here, the import receptors become the most rate-limiting factor for transport upon depletion of Nup358. Overexpression of the import 
receptors can compensate for the loss of Nup358 by increasing the local concentration of importins at the pore.

With respect to these findings, inhibition of nuclear import rather than accelerated export seems the more likely explanation although a decrease of CRM1-mediated export in cells with reduced levels of Nup358 was reported (Bernad et al., 2004). However, a direct involvement of Nup358 in CRM1-mediated export is rather unlikely (Hutten et al., 2006). Together, Nup358 appears to serve as an assembly/disassembly platform for the recycling importin/RanGTP complex and for newly formed import complexes. After hydrolysis of RanGTP by the pore bound RanGAP1 and in association with one of the four RanBDs of Nup358, the NTR stays in proximity of the NPC waiting for appropriate import cargos resulting in very efficient nuclear import. After depletion of Nup358, soluble RanGAP1 and RanBP1 can partially fulfill these tasks (Hutten et al., 2008) demonstrating that soluble RanGAP1 can fully substitute for the pore-associated protein.

\subsubsection{Nuclear import of a subset of proteins depends on Nup358}

Nup358 is involved in nuclear transport of artificial reporter proteins with well-defined NESs and NLSs (Hutten et al., 2008; Hutten et al., 2009). Does this dependency also apply to natural, cellular proteins? Indeed, the HIV-1 Rev protein, which is imported by transportin, accumulates in the cytoplasm in Nup358-depleted cells (Hutten et al., 2009). Here, accelerated export by CRM1 can be excluded (Hutten et al., 2009). Moreover, in a screen with 200 nuclear proteins derived from the LIFEdb* (database for localization, interaction, functional assays and expression of proteins, (Bannasch et al., 2004), a subset of proteins showed strong cytoplasmic accumulation after depletion of Nup358 (Hutten, 2007).

This database contains information about novel human open reading frames (ORF) and functional information on the encoded proteins gained by functional genomics and

* LIFEdb, DKFZ Heidelberg: http://www.dkfz.de/LIFEdb/(ih1dtsaptesp3h55w3jm1u55)/LIFEdb.aspx) 
proteomic approaches. Hence, certain motifs and signal sequences were identified by homology searches using BLAST. 


\subsection{Aim of the work}

The giant nucleoporin Nup358/RanBP2 with its multi-domain architecture is the major component of the cytoplasmic filaments of the NPC (Wu et al., 1995; Yokoyama et al., 1995). Some of these domains like the FG-repeats, which interact with NTRs or the four RanBDs, suggest an involvement in nuclear transport, and might function as an assembly/disassembly platform for nuclear transport complexes.

It had been reported, however, that nuclear import of reporter proteins containing a cNLS (using the importin $\alpha / \beta$ transport pathway) or an M9-sequence (interacting with the importin $\beta$ like import receptor transportin) do not require Nup358 (Walther et al., 2002; Hutten et al., 2006). On the other hand, depletion of Nup358 via RNA interference significantly slowed down import kinetics of cargos using various nuclear import pathways (Hutten et al., 2008; Hutten et al., 2009). Among these substrates that are retained in the cytoplasm upon Nup358 depletion are the viral protein HIV-1 Rev (Hutten et al., 2009), two DEAD-box helicases (DDX43 and DDX59), the SRY-related transcription factor Sox 6 and the putative tumor suppressor DBC-1 (deleted in breast cancer 1) (Hutten, 2007). The majority of nuclear proteins, by contrast, do not require Nup358 for efficient import (unpublished data).

In the first part of this work, the nuclear import pathways of the Nup358-dependent proteins DDX43, DDX59, Sox 6 and DBC-1 should be analyzed with respect to soluble transport factors.

The main part of the project addresses the role of Nup358 in nuclear import. DBC-1, the HIV-1 Rev and two reporter proteins using either the importin $\alpha / \beta$ or transportin pathway will be investigated. In particular, the responsible domain of the nucleoporin mediating nuclear import of these substrates should be identified and characterized. Rescue experiments with exogenous full-length Nup358 and several truncation mutants will be performed in cells depleted of endogenous Nup358 to analyze their ability to mediate nuclear import.

Together, we hope to shed light onto the cargo/Nup358-specific import pathway and to gain more insight into the role of Nup358 in nuclear transport. 



\section{MATERIAL \& METHODS}

\subsection{Material}

\subsubsection{Chemicals, reagents and enzymes}

Standard chemicals and buffer substances were obtained from AppliChem (Darmstadt), Carl Roth (Karlsruhe), Serva (Heidelberg), Sigma-Aldrich (Taufkirchen) and Merck (Darmstadt). Specific chemicals, reagents and enzymes are listed below:

\begin{tabular}{ll} 
Chemicals, reagents and enzymes & Company \\
\hline Acrylamid (30\%) & AppliChem \\
Aprotinin & Biomol \\
ATP & Sigma-Aldrich \\
$\beta$-Mercapthoethanol & Roth \\
BSA, fraction V & PAA \\
Calf intestinal phosphatase (CIP) & Fermentas \\
CO ${ }_{2}$-independent medium & Gibco \\
Creatine phosphate & Calbiochem \\
Creatine phosphate kinase & Calbiochem \\
Dexamethasone & Sigma-Aldrich \\
Digitonin & Calbiochem \\
Dimethylsulfoxide (DMSO) & \\
(cell culture grade) & AppliChem \\
Dithiothreitol (DTT) & AppliChem \\
DMEM (high glucose) & Gibco \\
DNA ladder, 1 kb & Fermentas \\
dNTPs & Fermentas \\
ECL & Milipore \\
FCS & Gibco \\
Fluorescence mounting medium & Dako Cytomation, Linaris \\
\hline
\end{tabular}


Chemicals, reagents and enzymes

GDP, GTP

Glutamine (cell culture grade)

Hoechst 33258

IPTG

Klenow-fragment

Leupeptin

Oligofectamine

Oligonucleotides

OptiMEM

Pefa bloc

Penicillin

Pepstatin

Phusion polymerase

PMSF

Poly-L-lysine

Protein ladder PAGE ruler (unstained, prestained)

Protein ladder SeeBlue2

Restriction enzymes

RNase A

Sequencing mix and buffer

siRNA oligonucleotides

Streptomycin

T4 DNA ligase

T4 polynucleotide kinase

Trypane blue

Trypsin/EDTA

Vent polymerase

\section{Company}

Sigma-Aldrich

Gibco

Sigma-Aldrich

Fermentas

Fermentas

Biomol

Invitrogen

Operon, Sigma-Aldrich

Gibco

Roth, Sigma-Aldrich

PAA

Biomol

Finnzymes, New England Biolabs

Sigma-Aldrich

Sigma-Aldrich

Fermentas

Invitrogen

Fermentas, New England Biolabs

AppliChem

Applied Biosystems

Ambion

PAA

Fermentas

Fermentas

Fluka

Gibco, PAA

New England Biolabs 


\subsubsection{Consumables}

Consumables were obtained from various common suppliers; some selected ones are listed below:

\section{Consumables}

Autoradiography films

Cell culture consumables

Centrifugal filter units

GFP-nanotrap

Glutathione sepharoseTM High Performance

LabTekTM chambered cover glass, 4 well

1.0 Borosilicate

Microscope slides (76×26×1 mm)

Microscope cover slips

1.0 Borosilicate

Protein $A$ agarose, Protein $\mathrm{G}$ agarose

PROTRAN nitrocellulose

Sterile filters and membranes

$(0.22-0.45 \mu \mathrm{M})$

\section{Company}

GE Healthcare, Kodak

Sarstedt, TPP

Millipore, Vivaspin

Chromotek

GE Healthcare

Nunc

Marienfeld

Marienfeld

Roche

Schleicher \& Schuell

Millipore, Pall, Renner, Sartorius

\subsubsection{Kits}

Kits

BigDye Terminator v1.1

cycle sequencing kit

NucleoBond ${ }^{\circledR}$ PC100, PC500

NucleoSpin ${ }^{\circledR}$ Extract II

\section{Company}

Applied Biosystems

Macherey \& Nagel

Macherey \& Nagel 


\subsubsection{Buffers, stock solutions and media}

Buffers and stock solutions were prepared using deionized water. Stock solutions were prepared freshly or stored at $-20^{\circ} \mathrm{C}$ if possible. The solutions were sterilized by autoclaving at $121^{\circ} \mathrm{C}$ for $20 \mathrm{~min}$. Solutions with heat-sensitive substances were sterilized by filtration through a $0.2 \mu \mathrm{m}$ filter. HEPES buffers were titrated with $\mathrm{KOH}$; other buffers were titrated with $\mathrm{NaOH}$ and $\mathrm{HCl}$.

\begin{tabular}{|c|c|}
\hline Buffer & \\
\hline $\begin{array}{l}\text { Annealing buffer } \\
(2 x)\end{array}$ & 80 mM Tris- $\mathrm{HCl}(\mathrm{pH}$ 7.5), 40 mM MgCl 2,100 mM NaCl \\
\hline Buffer A & $\begin{array}{l}50 \mathrm{mM} \text { Tris- } \mathrm{HCl}(\mathrm{pH} 7.5), 200 \mathrm{mM} \mathrm{NaCl}, 0.4 \% \text { Triton X-100, } \\
1 \mathrm{mM} \mathrm{MgCl} 2,5 \% \text { glycerol, freshly added } 2 \mathrm{mM} \mathrm{DTT} \text { and } 1 \mu \mathrm{g} / \mathrm{ml} \text { of } \\
\text { each protease inhibitor aprotinin, leupeptin and pepstatin }\end{array}$ \\
\hline Buffer B & $\begin{array}{l}50 \mathrm{mM} \text { Tris- } \mathrm{HCl}(\mathrm{pH} 7.5), 200 \mathrm{mM} \mathrm{NaCl}, \\
1 \mathrm{mM} \mathrm{MgCl} 2,5 \% \text { glycerol, freshly added } 2 \mathrm{mM} \mathrm{DTT} \text { and } 1 \mu \mathrm{g} / \mathrm{ml} \text { of } \\
\text { each protease inhibitor aprotinin, leupeptin and pepstatin }\end{array}$ \\
\hline $\begin{array}{l}\text { Colloidal coomassie } \\
\text { dye }\end{array}$ & $\begin{array}{l}0.1 \%(v / v) \text { brilliant blue G-250, } 2 \%(w / v) \text { ortho-phosphoric acid, } \\
10 \%(w / v)\left(\mathrm{NH}_{4}\right)_{2} \mathrm{SO}_{4}\end{array}$ \\
\hline $\begin{array}{l}\text { Coomassie } \\
\text { fixing solution }\end{array}$ & $45 \%(v / v)$ methanol, $10 \%(v / v)$ acetic acid \\
\hline $\begin{array}{l}\text { Coomassie } \\
\text { staining solution }\end{array}$ & $10 \%(v / v)$ acetic acid, $0.025 \%(w / v)$ brilliant blue G-250 \\
\hline $\begin{array}{l}\text { DNA loading dye } \\
(10 x)\end{array}$ & $\begin{array}{l}50 \%(\mathrm{v} / \mathrm{v}) \text { glycerol, } 0.1 \%(\mathrm{w} / \mathrm{v}) \text { bromphenol blue, } \\
0.1 \%(\mathrm{w} / \mathrm{v}) \text { xylencyanol }\end{array}$ \\
\hline $\begin{array}{l}\text { GFP-nanotrap } \\
\text { dilution buffer }\end{array}$ & $\begin{array}{l}10 \mathrm{mM} \text { Tris- } \mathrm{HCl}(\mathrm{pH} 7.5), 150 \mathrm{mM} \mathrm{NaCl}, 0.5 \mathrm{mM} \text { EDTA, freshly } \\
\text { supplemented with } 2 \mathrm{mM} \text { DTT, } 1 \mathrm{mM} \text { PMSF, } 1 \mu \mathrm{g} / \mathrm{ml} \text { aprotinin, } \\
\text { leupeptin and pepstatin }\end{array}$ \\
\hline $\begin{array}{l}\text { GFP-nanotrap } \\
\text { lysis buffer }\end{array}$ & $\begin{array}{l}10 \mathrm{mM} \text { Tris- } \mathrm{HCl}(\mathrm{pH} 7.5), 150 \mathrm{mM} \mathrm{NaCl}, 0.5 \mathrm{mM} \text { EDTA, } \\
0.5 \% \mathrm{NP} 40 \text {, freshly supplemented with } 2 \mathrm{mM} \text { DTT, } 1 \mathrm{mM} \text { PMSF, } \\
1 \mu \mathrm{g} / \mathrm{ml} \text { aprotinin, leupeptin and pepstatin }\end{array}$ \\
\hline
\end{tabular}




\begin{tabular}{|c|c|}
\hline Buffer & \\
\hline $\begin{array}{l}\text { GFP-nanotrap } \\
\text { wash buffer }\end{array}$ & $\begin{array}{l}10 \mathrm{mM} \text { Tris- } \mathrm{HCl}(\mathrm{pH} 7.5), 300 \mathrm{mM} \mathrm{NaCl}, 0.5 \mathrm{mM} \text { EDTA, } \\
0.25 \% \mathrm{NP} 40 \text {, freshly supplemented with } 2 \mathrm{mM} \text { DTT, } 1 \mathrm{mM} \text { PMSF, } \\
1 \mu \mathrm{g} / \mathrm{ml} \text { aprotinin, leupeptin and pepstatin }\end{array}$ \\
\hline HBS (2x) (pH 6.98) & $50 \mathrm{mM}$ HEPES , $250 \mathrm{mM} \mathrm{NaCl}, 1.5 \mathrm{mM} \mathrm{Na}_{2} \mathrm{HPO}_{4}$ \\
\hline $\begin{array}{l}\text { Laemmli } \\
\text { running buffer }\end{array}$ & 25 mM Tris, 192 mM glycine, $0.01 \%$ (w/v) SDS \\
\hline NP40-buffer & 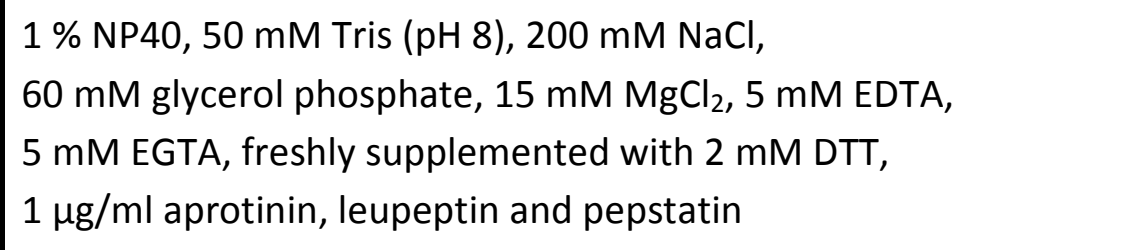 \\
\hline Buffer P1 & $50 \mathrm{mM}$ Tris- $\mathrm{HCl}(\mathrm{pH}$ 8), $10 \mathrm{mM}$ EDTA, $100 \mu \mathrm{g} / \mathrm{ml}$ RNase A \\
\hline Buffer P2 & $200 \mathrm{mM} \mathrm{NaOH}, 1 \%(\mathrm{v} / \mathrm{v}) \mathrm{SDS}$ \\
\hline Buffer P3 & $3 \mathrm{M} \mathrm{KOAc}(\mathrm{pH} 5.5)$ \\
\hline $\begin{array}{l}\text { Phosphate buffered } \\
\text { saline (PBS) }\end{array}$ & $\begin{array}{l}140 \mathrm{mM} \mathrm{NaCl}, 2.7 \mathrm{mM} \mathrm{KCl}, 10 \mathrm{mM} \mathrm{Na}_{2} \mathrm{HPO}_{4} \text {, } \\
1.5 \mathrm{mM} \mathrm{KH}_{2} \mathrm{PO}_{4}(\mathrm{pH} \text { 7.5) }\end{array}$ \\
\hline PBS-Tween & PBS supplemented with $0.1 \%(v / v)$ Tween 20 \\
\hline Ponceau S & $0.5 \%(\mathrm{w} / \mathrm{v})$ Ponceau S, $1 \%(\mathrm{v} / \mathrm{v})$ acetic acid \\
\hline $\begin{array}{l}\text { SDS-PAGE } \\
\text { loading buffer }(4 x)\end{array}$ & $\begin{array}{l}4 \%(\mathrm{w} / \mathrm{v}) \text { SDS, } 125 \mathrm{mM} \text { Tris (pH 6.8), } 10 \%(\mathrm{v} / \mathrm{v}) \text { glycerol, } \\
0.02 \%(\mathrm{w} / \mathrm{v}) \text { bromphenol blue, } 10 \%(\mathrm{v} / \mathrm{v}) \beta \text {-mercaptoethanol }\end{array}$ \\
\hline $\begin{array}{l}\text { Silver gel } \\
\text { developing solution }\end{array}$ & $\begin{array}{l}3 \%(\mathrm{w} / \mathrm{v}) \mathrm{Na}_{2} \mathrm{CO}_{3}, 0.018 \%(\mathrm{v} / \mathrm{v}) \text { formaldehyde, } \\
0.0005 \%(\mathrm{w} / \mathrm{v}) \mathrm{Na}_{2} \mathrm{~S}_{2} \mathrm{O}_{3}\end{array}$ \\
\hline $\begin{array}{l}\text { Silver gel } \\
\text { fixing solution }\end{array}$ & $\begin{array}{l}50 \%(v / v) \text { ethanol, } 12 \%(v / v) \text { acetic acid, } \\
0.018 \%(w / v) \text { formaldehyde }\end{array}$ \\
\hline TAE-buffer & $40 \mathrm{mM}$ Tris acetate (pH 7.7), $1 \mathrm{mM}$ EDTA \\
\hline TE-buffer & $10 \mathrm{mM}$ Tris-HCl (pH 7.4), 1 mM EDTA \\
\hline TFB-I-buffer & $100 \mathrm{mM} \mathrm{RbCl}, 15 \%$ (v/v) glycerol, $0.5 \mathrm{mM}$ LiCl (pH 5.8) \\
\hline
\end{tabular}




\begin{tabular}{l|l}
\multicolumn{1}{|l|}{ Buffer } & \\
\hline TFB-II-buffer & $\begin{array}{l}10 \mathrm{mM} \text { MOPS (pH 7), } 10 \mathrm{mM} \mathrm{RbCl}, 75 \mathrm{mM} \mathrm{CaCl}_{2}, \\
15 \%(\mathrm{v} / \mathrm{v}) \text { glycerol }\end{array}$ \\
& $\begin{array}{l}20 \mathrm{mM} \text { Hepes }(\mathrm{pH} 7.3), 110 \mathrm{mM} \text { KOAc, } 2 \mathrm{mM} \mathrm{Mg}(\mathrm{OAc})_{2}, \\
1 \mathrm{mM} \text { EDTA, freshly supplemented with } 2 \mathrm{mM} \mathrm{DTT}, 1 \mu \mathrm{g} / \mathrm{ml}\end{array}$ \\
$\begin{array}{l}\text { Transport buffer } \\
\text { (TB) }\end{array}$ & $\begin{array}{l}25 \mathrm{mM} \text { Tris-HCl, } 192 \mathrm{mM} \text { glycine, } 20 \%(\mathrm{v} / \mathrm{v}) \text { methanol, } \\
\text { Western blot } \\
\text { transfer buffer }\end{array}$ \\
\end{tabular}

\section{Stock solutions}

\begin{tabular}{ll}
\hline Ampicillin & $100 \mathrm{mg} / \mathrm{ml}$ \\
Aprotinin, 1000x & $1 \mathrm{mg} / \mathrm{ml}$ \\
ATP & $100 \mathrm{mM}$ in $100 \mathrm{mM} \mathrm{Mg}(\mathrm{OAc})_{2}$, \\
& $20 \mathrm{mM} \mathrm{HEPES}(\mathrm{pH} 7.4)$ \\
Chloramphenicol & $30 \mathrm{mg} / \mathrm{ml}$ \\
Dexamethasone & $2.5 \mathrm{mM}$ \\
Digitonin & $10 \%(\mathrm{w} / \mathrm{v})$ in DMSO \\
Dithiothreitol (DTT) & $1 \mathrm{M}$ \\
Hoechst 33258 & $10 \mathrm{mg} / \mathrm{ml}$ \\
Kanamycin & $50 \mathrm{mg} / \mathrm{ml}$ \\
Leupeptin/Pepstatin, 1000x & $1 \mathrm{mg} / \mathrm{ml}$ each, in DMSO \\
Pefa bloc, 100x & $100 \mathrm{mM}$ \\
Phenylmethylsulfonyl fluoride (PMSF) & $100 \mathrm{mM}$ in 2-propanol \\
Penicillin-Streptomycin, 1000x & Penicillin 10,000 U/ml \\
& Streptomycin 10 mg/ml
\end{tabular}




\section{Bacterial medium}

LB

$1 \%(\mathrm{w} / \mathrm{v})$ bacto-tryptone, $0.5 \%(\mathrm{w} / \mathrm{v})$ yeast extract, $1 \%(\mathrm{w} / \mathrm{v}) \mathrm{NaCl}(\mathrm{pH} 7)$

LB agar plates

LB supplemented with $1.5 \%(\mathrm{w} / \mathrm{v})$ bacto-agar

SOC

$2 \%(\mathrm{w} / \mathrm{v})$ tryptone, $5 \%(\mathrm{w} / \mathrm{v})$ yeast extract, $50 \mathrm{mM} \mathrm{NaCl}$, $2.5 \mathrm{mM} \mathrm{KaCl}, 10 \mathrm{mM} \mathrm{MgCl}_{2}, 10 \mathrm{mM} \mathrm{MgSO}_{4}$

Cell culture medium

\begin{tabular}{ll}
\hline & High Glucose $(4500 \mathrm{mg} / \mathrm{ml}), 10 \%(\mathrm{v} / \mathrm{v}) \mathrm{FCS}$, \\
& $2 \mathrm{mM}$ glutamine, $100 \mathrm{U} / \mathrm{ml}$ penicillin, \\
& $100 \mu \mathrm{g} / \mathrm{ml}$ streptomycin \\
$\mathrm{CO}_{2}$-independent medium & $10 \%(\mathrm{v} / \mathrm{v}) \mathrm{FCS}$
\end{tabular}

\subsubsection{Technical equipment and software}

General laboratory equipment was obtained from various common suppliers; some selected ones are listed below:

\begin{tabular}{ll} 
Technical equipment & Company \\
\hline Axiovert 200 M & Carl Zeiss \\
Centrifuge Allegra X-15R & Beckman Coulter \\
Centrifuge Avanti J-30I & Beckman Coulter \\
Confocal microscope LSM 510 meta & Carl Zeiss \\
Delta vision & Applied Precision \\
Documentation system LAS 3000 & Fujifilm \\
Emulsi flex-C5 & Avestin \\
Film developing machine Curix 60 & Agfa \\
Olympus IX 71 & Olympus \\
Rotors JA 30.50Ti, SX4750, TLA 100.3 & Beckman Coulter \\
Scanner 4990 Photo & Epson
\end{tabular}


Technical equipment

Table centrifuges 5415 D, 5415 R, 5424

Thermocycler Gene Amp PCR 2400

Thermocycler Primus MWG

Thermocycler Tprofessional Gradient,

Standard

Ultracentrifuge OptimaMax

UV cross linker

\section{Company}

Eppendorf

Perkin Elmer

Biotech

Biometra

Beckman Coulter

Biometra

\section{Software}

AxioVision (LE) Rel. 4.8

BLAST

Chromas Lite 2.01

Delta vision software

Endnote 9.0

FASTA Protein Similarity Search

Image Reader LAS 3000

LSM image browser

LSM software

MAFFT alignment

MUSCLE alignment

Nucleic Acid Sequence Massager

Photoshop 6.0

Vector NTI

\section{Company/Origin}

Carl Zeiss

http://blast.ncbi.nlm.nih.gov/

Technelysium

Applied Precision

Thomson Reuters

http://www.ebi.ac.uk/Tools/fasta33

Fujifilm

Carl Zeiss

Carl Zeiss

http://mafft.cbrc.jp/alignment/server/

http://www.ebi.ac.uk/Tools/muscle/index.h tml

http://www.attotron.com/cybertory/analysi s/seqMassager.htm

Adobe

Invitrogen 


\subsubsection{Cell lines}

Mammalian cell lines

\begin{tabular}{lll} 
Name & Resource & Description \\
\hline HeLa P4-R5 MAGI & $\begin{array}{l}\text { NIH AIDS Research and } \\
\text { Reference Reagent Program } \\
\text { Catalog number 3580 }\end{array}$ & $\begin{array}{l}\text { adherent human cervix carcinoma } \\
\text { cells (Charneau et al., 1994) }\end{array}$ \\
293T HEK & ATCC, CRL-2828 & $\begin{array}{l}\text { adherent human embryonic kidney } \\
\text { cells }\end{array}$
\end{tabular}

\section{Bacterial strains}

\begin{tabular}{ll} 
Name & Purpose \\
\hline E. coli DH5 $\alpha$ & Common cloning and DNA preparation \\
E. coli One Shot & Generated DNA is not methylated, used for methylation-sensitive \\
INV110 & restriction enzymes \\
E. coli Rosetta & Protein expression
\end{tabular}

\subsubsection{SiRNA}

The Nup358 siRNA was synthesized with standard purity by Ambion. After dilution to $100 \mu \mathrm{M}$ with RNase-free water, the siRNA was stored at $-80^{\circ} \mathrm{C}$.

\begin{tabular}{l|lll} 
Name & siRNA sequence 5' $\rightarrow \mathbf{3}^{\prime}$ & $\begin{array}{l}\text { target } \\
\text { sequence }\end{array}$ & Reference \\
\hline \multirow{2}{*}{ siRNA Nup358-1 } & CACAGACAAAGCCGUUGAAUU & $\begin{array}{l}\text { nct 351-369 } \\
\text { accession no } \\
\text { NM_006267 }\end{array}$
\end{tabular}




\subsubsection{Oligonucleotides}

Oligonucleotides designed in the course of this work were obtained from Sigma-Aldrich or Operon.

\section{Oligonucleotides for mutagenesis}

Red letters indicate the mutated nucleotides. Underlined letters indicate the position of the deletion.

\begin{tabular}{|c|c|c|}
\hline$\#$ & Name & Sequence $5^{\prime} \rightarrow$ 3' \\
\hline 402 & Nup358_RNAi_resc_f & GGAAGAAAACACAGATAAGGCCGTTGAATGTTACAGGC \\
\hline 403 & Nup358_RNAi_resc_r & GCCTGTAACATTCAACGGCCTTATCTGTGTTTTCTTCC \\
\hline 508 & DBC-1_KR201/202AA_f & GACTATGACTCCAAGGCAGCCAAACAGCGGGCTGGTG \\
\hline 509 & DBC-1_KR201/202AA_r & CACCAGCCCGCTGTTTGGCTGCCTTGGAGTCATAGTC \\
\hline 543 & Sox 6_K642A/P644A_f & CAATGTCCAACCAGGAGGCGCAAGCTTATTATGAAGAGCAGGC \\
\hline 544 & Sox 6_K642A/P644A_r & GCCTGCTCTTCATAATAAGCTTGCGCCTCCTGGTTGGACATTG \\
\hline 553 & Sox 6_G631A/R633A_f & CCAACATTAGCAAAATCTTAGCATCTGCCTGGAAATCAATGTCCAACC \\
\hline 554 & Sox 6_G631A/R633A_r & GGTTGGACATTGATTTCCAGGCAGATGCTAAGATTTTGCTAATGTTGG \\
\hline 582 & Sox 6_R614A_f & GGGCAAAGGATGAGAGGGCAAAAATCCTTCAGGCCTTCCCC \\
\hline 583 & Sox 6_R614A_r & GGGGAAGGCCTGAAGGATTTTTGCCCTCTCATCCTTTGCCC \\
\hline 575 & Sox 6_RK615/616AA_f & GGGCAAAGGATGAGAGGGCAGCAATCCTTCAGGCCTTCCCC \\
\hline 576 & Sox 6_RK615/616AA_r & GGGGAAGGCCTGAAGGATTGCTGCCCTCTCATCCTTTGCCC \\
\hline 571 & Nup358_Stopp_IR1_f & ACAGCTGTCAAGAAACTTTAAGGAAAACTATATTTG \\
\hline 572 & Nup358_Stopp_IR1_r & CAAATATAGTTTTCCTTAAAGTTTCTTGACAGCTGT \\
\hline 598 & DBC-1__aa243-264_f & CTGTGACTTCCTAGAACTCTCAGCCTTCCCCCTGAGC \\
\hline 599 & DBC-1__aa243-264_r & GCTCAGGGGGAAGGCTGAGAGTTCTAGGAAGTCACAG \\
\hline 633 & Sox 6_R600G_f & GCGAGCCACACATTAAGGGACCAATGAATGCATTCATG \\
\hline 634 & Sox 6_R600G_r & CATGAATGCATTCATTGGTCCCTTAATGTGTGGCTCGC \\
\hline 691 & Nup358_F1141A_f & CCAGGGAAATCAGTAGCTGGAACACCCACTTTAG \\
\hline 692 & Nup358_F1141A_r & CTAAAGTGGGTGTTCCAGCTACTGATTTCCCTGG \\
\hline 708 & $\begin{array}{l}\text { Sox } 6 \\
\text { _aa606_R600G_Xhol_r }\end{array}$ & TTTTCTCGAGAACATGAATGCATTCATTGGTCC \\
\hline
\end{tabular}




\section{Oligonucleotides for cloning}

\begin{tabular}{|c|c|c|}
\hline \# & Name & Sequence $5^{\prime} \rightarrow 3^{\prime}$ \\
\hline 198 & Nup358_Ncol_f & TTTTCCATGGAAATGAGGCGCAGCAAGGCT \\
\hline 201 & Nup358_aa1133_Clal_r & TTTTATCGATTCAAAACATATCATCACTTCGCCGA \\
\hline 202 & Nup358_aa805_Clal_r & TTTTATCGATTCATTCTGCCCATCGAGGTGG \\
\hline 203 & Nup358_aa2307_Ncol_f & TTTTCCATGGGACAGTACTTTGAACCTGTTGTTC \\
\hline 204 & Nup358_aa3047_Clal_r & TTTTATCGATTCAGAGTTTCATTAAATTCTGCTGACA \\
\hline 205 & Nup358_aa2710_Clal_r & TTTTATCGATTCAACATTCTTTCTCATTATCTGCTG \\
\hline 419 & MCS_rescue_Notl/Sall_f & CGATAACGCGGCCGCAACGTCGACAACA \\
\hline 420 & MCS_rescue_Notl/Sall_r & CTAGTACCGTCGACACCGCGGCCGCACCAT \\
\hline 474 & Nup358_aa1133_Clal_r & TTTTATCGATAAACATATCATCACTTCGCCGA \\
\hline 501 & Imp a_Ncol_f & TTTTCCATGGAAATGTCCACCAACGAGAATGC \\
\hline 502 & Imp a_Spel_r & TTTTACTAGTTCAAAAGTTAAAGGTCCCAGGAGC \\
\hline 517 & Sirt1_Sall_f & TTTTGTCGACATGGCGGACGAGGCG \\
\hline 518 & Sirt1_aa747_BamHI_r & TTTTGGATCCTGATTTGTTTGATGGATAG \\
\hline 526 & Sox 6_BgllI_f & TTTTAGATCTATGGGAAGAATGTCTTCCAAGC \\
\hline 527 & Sox 6_Sall_r & TTTTGTCGACAAGTTGGCACTGACAGCCTCC \\
\hline 573 & Sox 6_aa456_Start_Notl_f & TTTTGCGGCCGCATGATCCTATCTAGTCTCAAC \\
\hline 574 & Sox 6_Xhol_r & TTTTCTCGAGAAGTTGGCACTGACAGCCTC \\
\hline 586 & DBC-1_Xhol_f & TTTTCTCGAGAAATGTCCCAGTTTAAGCGC \\
\hline 587 & DBC-1_aa220_EcoRI_r & TTTTGAATTCCTAGTCATGCCTTGGCTTCTTAG \\
\hline 588 & DBC-1_aa200_Xhol_f & TTTTCTCGAGAAAAGAAACGCAAACAGCG \\
\hline 589 & DBC-1_aa199_EcoRI_r & TTTTGAATTCCTAGGAGTCATAGTCATCACTTCG \\
\hline 590 & DBC-1_aa220_Xhol_f & TTTTCTCGAGAACTGCCTCCTTACCGGG \\
\hline 591 & DBC-1_EcoRI_r & TTTTGAATTCCTAGCTAGGTGCCGGCTC \\
\hline 596 & DBC-1_aa793_EcoR1_r & TTTTGAATTCCTAAGCACCTGGCTTCGTGC \\
\hline 600 & cNLS_f_Xhol & TCGAGAAGGGCCAAAGAAAAAGAGGAAAGTTTC \\
\hline 601 & cNLS_r_Xhol & TCGAGAAACTTTCCTCTTTTTCTTTGGCCCTTC \\
\hline 602 & DBC-1_HindIII_aa794_f & TTTTAAGCTTCGGCCCCCACAGAACACA \\
\hline 625 & Exp4_Spel_r & TTTTACTAGTTTATTTTACACAAAGGAGACCACC \\
\hline 628 & Nup358_EcoRV_intern_aa852_f & CTGATGGATATCAGGGG \\
\hline 629 & Nup358_Notl_aa2148_r & TTTTGCGGCCGCCTAGTCTAACAGAAGCCGCTG \\
\hline
\end{tabular}




\begin{tabular}{|c|c|c|}
\hline \# & Name & Sequence $5^{\prime} \rightarrow 3^{\prime}$ \\
\hline 630 & Nup358_Notl_aa2448_r & TTTTGCGGCCGCCTAAGAATCTTTTTCCTGGGC \\
\hline 649 & Exp4_Clal_f & TTTTATCGATGTCGACGCAGCAGCATTA \\
\hline 665 & Sox 6_aa601_Start_Notl_f & TTTTGCGGCCGCATGATGAATGCATTCATGG \\
\hline 666 & Sox 6_aa701_Xhol_r & TTTTCTCGAGAAAAAGAACTGCCTCATCTC \\
\hline 667 & Sox 6_aa702_Start_Notl_f & TTTTGCGGCCGCATGACTGTGGGGCAACAGCC \\
\hline 670 & Nup358_Notl_aa1810_r & TTTTGCGGCCGCCTAAGTTGGTTTAGAGGCATC \\
\hline 683 & Nup358_Notl_aa1170_r & TTTTGCGGCCGCTTTTAAAATGCTCTGGG \\
\hline 684 & Nup358_Notl_aa1306_r & TTTTGCGGCCGCAGGACCGTCATCATCATC \\
\hline 696 & Syne1_aa355_Ncol_f & TTTTCCATGGCCGCCTGGCTAGGAGAGACAG \\
\hline 697 & Syne1_Notl_r & TTTTGCGGCCGCGAGTGGAGGAGGACCGTT \\
\hline 706 & Nup358_aa1000_Spel & TTTTACTAGTCTATTCTATAGTCTTAGATTCTGCAGA \\
\hline 707 & Sox 6_aa606_Xhol_r & TTTTCTCGAGAACATGAATGCATTCATTGG \\
\hline 709 & Kpn21/BgllII_NotI_f & CCGGACTCAGCGGCCGCCTCA \\
\hline 710 & Kpn21/BgllII_Notl_r & GATCTGAGGCGGCCGCTGAGT \\
\hline 720 & Nup358_aa806_Notl_f & TTTTGCGGCCGCGGATCAGAATTCTTTACTGAAAATG \\
\hline 721 & Nup358_aa1306_Spel_r & TTTTACTAGTTTTTAAAATGCTCTGGGC \\
\hline 734 & Nup358_aa806_Ncol_f & TTTTCCATGGAAGATCAGAATTCTTTACTGAAAATG \\
\hline
\end{tabular}

\section{Sequencing}

Oligonucleotides designed and applied for cloning were used for sequencing, too.

\begin{tabular}{ll} 
Name & Sequence $\mathbf{5}^{\prime} \boldsymbol{\rightarrow} \mathbf{3}$ \\
\hline CMV-promotor & TCCAAGTCTCCACCCCATTG \\
GFP 3'_r & TCAGCTTGCCGTAGGTGGCATCGCCC \\
GFP 5'_f & CGAGAAGCGCGATCACAT \\
GFP MCS I 3'_r & CCACAACTAGAATGCAGTGAAAA \\
GFP MCS II 3'_r & TTGCATTCATTTTATGTTTCAGG \\
mCherry_f & GCCTACAACGTCAACATCAAGTTG \\
pEF-HA_f & GCCATCTATTGCTTACATTTGCTTCTGACACAACTG \\
pEF-HA_r & CCAGGGCATTAGCCACACCAGCCACCACTTTCTG
\end{tabular}




\begin{tabular}{ll} 
Name & Sequence $\mathbf{5}^{\prime} \boldsymbol{\rightarrow} \mathbf{3}$ \\
\hline pGEX_f & GGGCTGGCAAGCCACGTTTGGTG \\
pGEX_r & CCGGGAGCTGCAGTGGTCAGAGG \\
seq_Nup358_267_f & GTCTGTGAAATCTTTGGGTGG \\
seq_Nup358_534_r & CATCCCACCAAGATTTTTGTC \\
seq_Nup358_aa1048_f & CCACAGGTTGTGACACAGC \\
seq_DBC-1_Mut_f & CCTCTTCTGCATGTAGCAGC \\
seq_DBC-1_Mut_r & GAAAAGGGCTGGCTCAGGGG \\
seq_SRY6_mut_PY_r & GCTGGGCTCCGGGCTGG \\
T3-promotor & AATTAACCCTCACTAAAGG \\
T5-promotor & AATTTATTTGCTTTGTGAGC \\
T7-promotor & TAATACGACTCACTATAGGG \\
T7-terminator & CGATCAATAACGAGTCGC
\end{tabular}

\subsubsection{Vectors and plasmids}

\section{Available vectors}

\begin{tabular}{l|llll}
$\#$ & Name & Tag & Expression/Use & Source \\
\hline P 294 & pEGFP 2 -C1 & N-terminal 2xGFP & mammalian & Dr. S. Hutten \\
P 373 & pEGFP 2 -N1 & N-terminal 2xGFP & mammalian & Dr. S. Hutten \\
V 22 & pEGFP-N1 & C-terminal GFP & mammalian & Clontech \\
V 27 & pEF-HA (plink) & N-terminal 2xHA & mammalian & Dr. O. T. Fackler ${ }^{*}$ \\
V 30 & pEGFP-C1 & N-terminal GFP & mammalian & Clontech \\
V 46 & pGEX-6P-1 & N-terminal GST & bacterial & GE Healthcare \\
V 49 & pEF-HA_MCS & N-terminal 2xHA & mammalian & Dr. S. Hutten \\
V 51 & pcDNA 3.1 + & & mammalian & Invitrogen
\end{tabular}

\footnotetext{
* Prof. Dr. Oliver T. Fackler, Department of Virology, University of Heidelberg
} 


\begin{tabular}{l|llll}
$\#$ & Name & Tag & Expression/Use & Source \\
\hline V 61 & pmCherry-C1 & $\begin{array}{l}\text { N-terminal } \\
\text { mCherry }\end{array}$ & mammalian & Clontech \\
V 66 & pmRFP-C1 & N-terminal mRFP & mammalian & Clontech \\
V 68 & pBS II SK (-/+) & & cloning & Stratagen
\end{tabular}

\section{Generated vectors within this work}

\begin{tabular}{|c|c|c|}
\hline$\#$ & Name & Description \\
\hline P 766 & $p E G F P_{2}-c N L S$ & $\begin{array}{l}\text { pEGFP } 2 \text {-C1 (P 294) was enlarged for a cNLS, which does not } \\
\text { contain a stop codon. It was cloned via Xhol. }\end{array}$ \\
\hline V 64 & pEF-HA_MCS_2 & $\begin{array}{l}\text { The MCS was enlarged for Notl and Sall, which was } \\
\text { introduced via Clal and Spel. }\end{array}$ \\
\hline V 69 & pmRFP_MCS & $\begin{array}{l}\text { The MCS was enlarged for Not I, which was introduced via } \\
\text { BgllII and Kpn } 21 \text {. }\end{array}$ \\
\hline
\end{tabular}

\section{Available plasmids}

Tags are always located at the respective terminus shown in the plasmid construct.

\begin{tabular}{|c|c|c|c|c|}
\hline \# & Name & Tag & $\begin{array}{l}\text { Expression/ } \\
\text { Use }\end{array}$ & Source \\
\hline P 54 & pQE70-Rchl & C-terminal $6 x \mathrm{His}$ & bacterial & $\begin{array}{l}\text { Dr. D. Görlich } \\
\text { (Görlich et al., 1995a) }\end{array}$ \\
\hline P 234 & $\begin{array}{l}\text { pXRGG } \\
(\text { Rev-GR } \\
511-795-G F P)\end{array}$ & $\mathrm{GR}_{511-795}$-GFP & mammalian & $\begin{array}{l}\text { Dr. J. A. Hanover }{ }^{\dagger} \\
\text { (Love et al., 1998) }\end{array}$ \\
\hline P 296 & $\mathrm{pEGFP}{ }_{2}-\mathrm{cNLS}$ & $2 \times G F P$ & mammalian & $\begin{array}{l}\text { Dr. S. Hutten } \\
\text { (Hutten et al., 2006) }\end{array}$ \\
\hline P 318 & pGEX-2T-GST-Sox 6 & $\mathrm{~N}$-terminal GST & bacterial & Dr. S. Hutten \\
\hline
\end{tabular}

\footnotetext{
* Prof. Dr. Dirk Görlich, Max Planck Institute for Biophysical Chemistry, Goettingen

${ }^{\dagger}$ Dr. John A. Hanover, NIDKK Bethesda, MD, USA
} 


\begin{tabular}{|c|c|c|c|c|}
\hline \# & Name & Tag & $\begin{array}{l}\text { Expression/ } \\
\text { Use }\end{array}$ & Source \\
\hline P 324 & $\begin{array}{l}\text { pGEX-2T-GST- } \\
\text { DDX43 }\end{array}$ & $\mathrm{N}$-terminal GST & bacterial & Dr. S. Hutten \\
\hline P 325 & $\begin{array}{l}\text { pGEX-2T-GST- } \\
\text { DDX59 }\end{array}$ & $\mathrm{N}$-terminal GST & bacterial & Dr. S. Hutten \\
\hline P 334 & pEF-HA-DBC-1 & $2 x H A$ & mammalian & Dr. S. Hutten \\
\hline P 460 & pEF-HA-transportin & $2 x H A$ & mammalian & $\begin{array}{l}\text { Dr. S. Hutten } \\
\text { (Hutten et al., 2008) }\end{array}$ \\
\hline P 476 & pEF-HA-importin $\beta$ & $2 x \mathrm{HA}$ & mammalian & $\begin{array}{l}\text { Dr. S. Hutten } \\
\text { (Hutten et al., 2008) }\end{array}$ \\
\hline P 495 & pEF-HA-importin 7 & $2 x H A$ & mammalian & Dr. S. Hutten \\
\hline P 504 & $\operatorname{Rev}_{68-90}-G_{F P}-c N L S$ & 2xGFP & mammalian & $\begin{array}{l}\text { Dr. S. Hutten } \\
\text { (Hutten et al., 2008) }\end{array}$ \\
\hline P 631 & $\begin{array}{l}\left(\mathrm{GR}_{511-795}\right)_{2}-\mathrm{GFP}_{2}- \\
\mathrm{M} 9\end{array}$ & $2 \times G R, 2 x G F P$ & mammalian & $\begin{array}{l}\text { Dr. S. Hutten } \\
\text { (Hutten et al., 2009) }\end{array}$ \\
\hline P 651 & pcDNA4/TO-ER $\alpha$ & $\mathrm{N}$-terminal HA & mammalian & Dr. S. Johnson ${ }^{*}$ \\
\hline P 754 & Sirt1-myc & C-terminal myc & mammalian & Dr. T. Kouzarides ${ }^{\dagger}$ \\
\hline P 755 & CFP-DDX43 & $\mathrm{N}$-terminal CFP & mammalian & LIFEdb, DKFZ \\
\hline P 756 & DDX43-YFP & C-terminal YFP & mammalian & LIFEdb, DKFZ \\
\hline P 757 & CFP-DDX59 & $\mathrm{N}$-terminal CFP & mammalian & LIFEdb, DKFZ \\
\hline P 758 & DDX59-YFP & C-terminal YFP & mammalian & LIFEdb, DKFZ \\
\hline P 759 & Sox 6-YFP & C-terminal YFP & mammalian & LIFEdb, DKFZ \\
\hline P 760 & CFP-DBC-1 & $\mathrm{N}$-terminal CFP & mammalian & LIFEdb, DKFZ \\
\hline P 761 & DBC-1-YFP & C-terminal YFP & mammalian & LIFEdb, DKFZ \\
\hline P 762 & $\begin{array}{l}\text { pCS2+MT-Syne 1B } \\
\text { aa 1639-1989 }\end{array}$ & $\begin{array}{l}\text { N-terminal } \\
\text { FLAG }\end{array}$ & mammalian & $\begin{array}{l}\text { Dr. D. Starr }{ }^{\ddagger} \\
\text { (Grady et al., 2005) }\end{array}$ \\
\hline P 763 & UTF-1-YFP & C-terminal YFP & mammalian & LIFEdb, DKFZ \\
\hline P 764 & pBS-TKS-Nup358 & & cloning & (Yokoyama et al., 1995) \\
\hline P 765 & pQE30-exportin 4 & $\mathrm{~N}$-terminal $6 \mathrm{xHis}$ & bacterial & $\begin{array}{l}\text { Dr. D. Görlich }{ }^{\S} \\
\text { (Lipowsky et al., 2000) }\end{array}$ \\
\hline
\end{tabular}

\footnotetext{
* Dr. Steven Johnson, Molecular Oncology, Ernst-August University of Goettingen. Plasmid was originally made by David G. Monroe, Mayo Clinic, Rochester, MN, USA.

' Prof. Tony Kouzarides, The Gurdon Institute, Cambridge, UK

‡ Dr. Daniel Starr, UC Davis, Davis, CA, USA

$\S$ Prof. Dr. Dirk Görlich, Max Planck Institute for Biophysical Chemistry, Goettingen
} 


\section{Generated plasmids within this work}

Tags are always located at the respective terminus shown in the plasmid construct. TEMPLATE DNA was amplified by PCR with the indicated oligonucleotides (oligo), digested with the respective enzymes, and ligated into VECTOR. If no oligos are indicated, the new plasmid was designed by digesting the TEMPLATE DNA, and by ligating the insert into the digested VECTOR.

\begin{tabular}{|c|c|c|c|c|}
\hline \# & Name & $\begin{array}{l}\# \\
\text { Oligo }\end{array}$ & $\begin{array}{l}\text { Template } \\
\text { Vector }\end{array}$ & $\begin{array}{l}\text { Restriction sites/ } \\
\text { description }\end{array}$ \\
\hline P 540 & $\begin{array}{l}\text { pEF-HA-Nup358 } \\
\text { aa 1-805 }\end{array}$ & $\begin{array}{l}201 \\
202\end{array}$ & $\begin{array}{l}\text { Nup358 (P 764) } \\
\text { pEF-HA }\end{array}$ & $\begin{array}{l}\text { Ncol } \\
\text { Clal }\end{array}$ \\
\hline P 541 & $\begin{array}{l}\text { pEF-HA-Nup358 } \\
\text { aa 2307-3047 }\end{array}$ & $\begin{array}{l}203 \\
204\end{array}$ & $\begin{array}{l}\text { Nup358 (P 764) } \\
\text { pEF-HA }\end{array}$ & $\begin{array}{l}\text { Ncol } \\
\text { Clal }\end{array}$ \\
\hline P 542 & $\begin{array}{l}\text { pEF-HA-Nup358 } \\
\text { aa } 2307-2710\end{array}$ & $\begin{array}{l}203 \\
205\end{array}$ & $\begin{array}{l}\text { Nup358 (P 764) } \\
\text { pEF-HA }\end{array}$ & $\begin{array}{l}\text { Ncol } \\
\text { Clal }\end{array}$ \\
\hline P 601 & $\begin{array}{l}\text { pEF-HA-Nup358 } \\
\text { aa } 806-1133\end{array}$ & $\begin{array}{l}734 \\
201\end{array}$ & $\begin{array}{l}\text { Nup358 (P 764) } \\
\text { pEF-HA }\end{array}$ & $\begin{array}{l}\text { Ncol } \\
\text { Clal }\end{array}$ \\
\hline P 731 & pEGFP-DBC-1 & & $\begin{array}{l}\text { pEF-HA-DBC-1 } \\
\text { pEGFP-C1 }\end{array}$ & Bsp 1407I \\
\hline & & $\begin{array}{l}198 \\
474\end{array}$ & $\begin{array}{l}\text { Nup358 (P 764) } \\
\text { pEF-HA_MCS2 }\end{array}$ & $\begin{array}{l}\text { Ncol } \\
\text { Clal }\end{array}$ \\
\hline P 732 & (siRNA-resistant) & $\begin{array}{l}402 \\
403\end{array}$ & $\begin{array}{l}\text { pEF-HA-Nup358 } \\
\text { aa } 1-1133\end{array}$ & $\begin{array}{l}\text { The mutations at nct } \\
231 \text { and } 235 \\
\text { introduced the } \\
\text { siRNA resistance. }\end{array}$ \\
\hline & & & $\begin{array}{l}\text { pEF-HA-Nup358 } \\
\text { aa 1-1133 (P 732) } \\
\text { pBS-TKS-Nup358 }\end{array}$ & $\begin{array}{l}\text { Ndel (nct 146) } \\
\text { Pmel (nct 1891) }\end{array}$ \\
\hline P 733 & pEF-HA Nup358 & \multicolumn{3}{|c|}{$\begin{array}{l}\text { pBS-TKS-Nup358* (P 790) was linearized by Xhol, } \\
\text { blunted by the Klenow-fragment, and further digested } \\
\text { by Notl. } \\
\text { pEF-HA_MCS2 was linearized by Spel, blunted by the } \\
\text { Klenow-fragment, and further digested by Notl. } \\
\text { Ligation was performed overnight and in presence of } \\
\text { PEG } 4000 \text {. }\end{array}$} \\
\hline
\end{tabular}




\begin{tabular}{|c|c|c|c|c|}
\hline$\#$ & Name & $\begin{array}{l}\text { \# } \\
\text { Oligo }\end{array}$ & $\begin{array}{l}\text { Template } \\
\text { Vector }\end{array}$ & $\begin{array}{l}\text { Restriction sites/ } \\
\text { description }\end{array}$ \\
\hline & & \multicolumn{3}{|c|}{$\begin{array}{l}\text { To be in frame, pEF-HA-Nup358 was linearized by Notl, } \\
\text { blunted by the Klenow-fragment, and an one-molecule } \\
\text { ligation was performed. }\end{array}$} \\
\hline P 739 & pEF-HA-exportin 4 & $\begin{array}{l}649 \\
625\end{array}$ & $\begin{array}{l}\text { exportin } 4 \text { (P 765) } \\
\text { pEF-HA }\end{array}$ & $\begin{array}{l}\text { Clal } \\
\text { Spel }\end{array}$ \\
\hline P 738 & $\begin{array}{l}\text { pEF-HA-importin } \alpha \\
\text { (human isoform 2) }\end{array}$ & $\begin{array}{l}501 \\
502\end{array}$ & $\begin{array}{l}\text { Rchl (P 54) } \\
\text { pEF-HA }\end{array}$ & $\begin{array}{l}\text { Ncol } \\
\text { Spel }\end{array}$ \\
\hline P 767 & pmCherry-Sirt1 & $\begin{array}{l}517 \\
518\end{array}$ & $\begin{array}{l}\text { Sirt1 (P 754) } \\
\text { pmCherry-C1 }\end{array}$ & $\begin{array}{l}\text { Sall } \\
\text { BamHI }\end{array}$ \\
\hline P 768 & Sox 6-pEGFP & $\begin{array}{l}526 \\
527\end{array}$ & $\begin{array}{l}\text { Sox 6-YFP (P 759) } \\
\text { pEGFP-N1 }\end{array}$ & $\begin{array}{l}\text { Bgl III } \\
\text { Sall }\end{array}$ \\
\hline P 769 & Sox 6 aa 1-455-pEGFP & & \multicolumn{2}{|c|}{$\begin{array}{l}\text { Sox 6-pEGFP (P 768) was digested by EcoRV } \\
\text { (nct 1368) and Sall (MCS), ends were blunted } \\
\text { by the Klenow-fragment, and an one-molecule } \\
\text { ligation in presence of PEG } 4000 \text { was } \\
\text { performed. }\end{array}$} \\
\hline P 770 & $\begin{array}{l}\text { Sox } 6 \text { aa } 456-804- \\
\text { pEGFP }\end{array}$ & $\begin{array}{l}573 \\
574\end{array}$ & $\begin{array}{l}\text { Sox } 6 \text { (P 768) } \\
\text { pEGFP } 2-N 1\end{array}$ & $\begin{array}{l}\text { Notl } \\
\text { Xhol }\end{array}$ \\
\hline P 771 & $\begin{array}{l}\text { Sox } 6 \text { aa } 601-700- \\
\text { pEGFP } 2\end{array}$ & $\begin{array}{l}665 \\
666\end{array}$ & $\begin{array}{l}\text { Sox } 6(P 768) \\
\text { pEGFP } 2-N 1\end{array}$ & $\begin{array}{l}\text { Notl } \\
\text { Xhol }\end{array}$ \\
\hline P 772 & $\begin{array}{l}\text { Sox } 6 \text { aa 701-804- } \\
\text { pEGFP } 2\end{array}$ & $\begin{array}{l}667 \\
574\end{array}$ & $\begin{array}{l}\text { Sox } 6 \text { (P 768) } \\
\text { pEGFP } 2-N 1\end{array}$ & $\begin{array}{l}\text { Notl } \\
\text { Xhol }\end{array}$ \\
\hline P 773 & $\begin{array}{l}\text { Sox } 6 \text { aa 456-606- } \\
\text { pEGFP } 2\end{array}$ & $\begin{array}{l}573 \\
707\end{array}$ & $\begin{array}{l}\text { Sox } 6 \text { (P 768) } \\
\text { pEGFP } 2-N 1\end{array}$ & $\begin{array}{l}\text { Notl } \\
\text { Xhol }\end{array}$ \\
\hline P 774 & $\begin{array}{l}\text { Sox } 6 \text { aa } 456-606 \\
\text { R600G-pEGFP } 2\end{array}$ & $\begin{array}{l}573 \\
708\end{array}$ & $\begin{array}{l}\text { Sox } 6 \text { (P 768) } \\
\text { pEGFP }-\mathrm{N} 1\end{array}$ & $\begin{array}{l}\text { Notl }+ \text { Xhol } \\
\text { The last nct of oligo } \\
\text { \# } 708 \text { contained the } \\
\text { mutation leading to } \\
\text { R600G. }\end{array}$ \\
\hline \multirow{3}{*}{ P 777} & \multirow{3}{*}{$\begin{array}{l}\text { Sox } 6 \text { aa } 601-700 \\
\text { G633A/R635A/K644A/ } \\
\text { P646A-pEGFP } 2\end{array}$} & $\begin{array}{l}543 \\
544\end{array}$ & Sox 6 (P 768) & mutagenesis \\
\hline & & $\begin{array}{l}553 \\
554\end{array}$ & $\begin{array}{l}\text { Sox } 6 \text { K644A/P646A } \\
\text { (P 775) }\end{array}$ & mutagenesis \\
\hline & & $\begin{array}{l}665 \\
666\end{array}$ & $\begin{array}{l}\text { Sox } 6 \\
\text { G633A/R635A/K644A/ } \\
\text { P646A (P 776) } \\
\text { pEGFP }_{2} \text {-N1 }\end{array}$ & $\begin{array}{l}\text { Notl } \\
\text { Xhol }\end{array}$ \\
\hline
\end{tabular}




\begin{tabular}{|c|c|c|c|c|}
\hline$\#$ & Name & $\begin{array}{l}\# \\
\text { Oligo }\end{array}$ & $\begin{array}{l}\text { Template } \\
\text { Vector }\end{array}$ & $\begin{array}{l}\text { Restriction sites/ } \\
\text { description }\end{array}$ \\
\hline \multirow{2}{*}{ P 779} & \multirow{2}{*}{$\begin{array}{l}\text { Sox } 6 \text { aa } 601-700 \\
\text { R614A/K615A-pEGFP } 2\end{array}$} & $\begin{array}{l}582 \\
583\end{array}$ & $\begin{array}{l}\text { pEGFP } 2 \text {-Sox } 6 \\
\text { aa } 601-700\end{array}$ & mutagenesis \\
\hline & & $\begin{array}{l}575 \\
576\end{array}$ & $\begin{array}{l}\text { pEGFP } 2 \text {-Sox } 6 \text { aa 601- } \\
700 \text { R614A (P 778) }\end{array}$ & mutagenesis \\
\hline P 780 & Sox 6 R600G-YFP & $\begin{array}{l}633 \\
634\end{array}$ & Sox 6-YFP & mutagenesis \\
\hline P 781 & $\begin{array}{l}\text { pEF-HA-DBC-1 } \\
\text { KR201/202AA }\end{array}$ & $\begin{array}{l}508 \\
509\end{array}$ & pEF-HA-DBC-1 & mutagenesis \\
\hline P 782 & $\begin{array}{l}\text { pEGFP-DBC-1 } \\
\text { KR201/202AA }\end{array}$ & & $\begin{array}{l}\text { pEF-HA-DBC-1 } \\
\text { KR201/202AA }\end{array}$ & Bsp1407I \\
\hline P 783 & $\begin{array}{l}\text { pEGFP } 2 \text {-DBC-1 } \\
\text { aa 1-199 }\end{array}$ & $\begin{array}{l}586 \\
589\end{array}$ & $\begin{array}{l}\mathrm{DBC}-1 \text { (P 731) } \\
\mathrm{pEGFP}_{2}-\mathrm{C} 1\end{array}$ & $\begin{array}{l}\text { Xhol } \\
\text { EcoRI }\end{array}$ \\
\hline P 784 & $\begin{array}{l}\text { pEGFP } 2-D B C-1 \\
\text { aa } 1-220\end{array}$ & $\begin{array}{l}586 \\
587\end{array}$ & $\begin{array}{l}\text { DBC-1 (P 731) } \\
\text { pEGFP }_{2}-\mathrm{C} 1\end{array}$ & $\begin{array}{l}\text { Xhol } \\
\text { EcoRI }\end{array}$ \\
\hline P 785 & $\begin{array}{l}\text { pEGFP-DBC-1 } \\
\text { aa } 200-923\end{array}$ & $\begin{array}{l}588 \\
591\end{array}$ & $\begin{array}{l}\text { DBC-1 (P 731) } \\
\text { pEGFP-C1 }\end{array}$ & $\begin{array}{l}\text { Xhol } \\
\text { EcoRI }\end{array}$ \\
\hline P 786 & $\begin{array}{l}\text { pEGFP-DBC-1 } \\
\text { aa } 220-923\end{array}$ & $\begin{array}{l}590 \\
591\end{array}$ & $\begin{array}{l}\text { DBC-1 (P 731) } \\
\text { pEGFP-C1 }\end{array}$ & $\begin{array}{l}\text { Xhol } \\
\text { EcoRI }\end{array}$ \\
\hline P 787 & $\begin{array}{l}\text { pEGFP-DBC-1 } \\
\Delta 243-264\end{array}$ & $\begin{array}{l}598 \\
599\end{array}$ & pEGFP-DBC-1 & mutagenesis \\
\hline P 788 & pEGFP-DBC-1 aa 1-793 & $\begin{array}{l}586 \\
796\end{array}$ & $\begin{array}{l}\text { DBC-1 (P 731) } \\
\text { pEGFP-C1 }\end{array}$ & $\begin{array}{l}\text { Xhol } \\
\text { EcoRI }\end{array}$ \\
\hline P 789 & $\begin{array}{l}\text { pEGFP }{ }_{2}-C N L S-D B C-1 \\
\text { aa } 794-923\end{array}$ & $\begin{array}{l}602 \\
591\end{array}$ & $\begin{array}{l}\text { DBC-1 (P 731) } \\
\text { pEGFP }_{2}-c N L S(P 766)\end{array}$ & $\begin{array}{l}\text { HindIII } \\
\text { EcoRI }\end{array}$ \\
\hline P 791 & $\begin{array}{l}\text { pEF-HA-Nup358 } \\
\text { aa 1-3047 }\end{array}$ & & $\begin{array}{l}\text { pEF-HA-Nup358 aa } 230 \\
\text { digested by Spel (nct } 7 \\
\text { resulting fragment con } \\
\text { after nct } 9141 \text { was clor } \\
\text { Nup358 which was dig } \\
\text { orientation of the inse } \\
\text { sequencing. }\end{array}$ & $\begin{array}{l}-3047 \text { (P 541) was } \\
78 \text { and MCS). The } \\
\text { ining a stop codon } \\
\text { d into pEF-HA- } \\
\text { ted by Spel. Correct } \\
\text { was analyzed by }\end{array}$ \\
\hline P 792 & $\begin{array}{l}\text { pEF-HA-Nup358 } \\
\text { aa 1-2684 }\end{array}$ & $\begin{array}{l}571 \\
572\end{array}$ & $\begin{array}{l}\text { In pEF-HA-Nup358 aa } \\
\text { stop codon was create } \\
\text { mutagenesis. }\end{array}$ & $\begin{array}{l}07-3047(P 541) \text { a } \\
\text { at nct } 8052 \text { by }\end{array}$ \\
\hline
\end{tabular}




\begin{tabular}{|c|c|c|c|c|}
\hline$\#$ & Name & $\begin{array}{l}\text { \# } \\
\text { Oligo }\end{array}$ & $\begin{array}{l}\text { Template } \\
\text { Vector }\end{array}$ & $\begin{array}{l}\text { Restriction sites/ } \\
\text { description }\end{array}$ \\
\hline & & & \multicolumn{2}{|c|}{$\begin{array}{l}\text { The resulting construct was digested by Spel } \\
\text { (nct } 7678 \text { and MCS). The insert containing a } \\
\text { stop codon at nct } 8052 \text { was cloned into pEF- } \\
\text { HA-Nup358 which was digested by Spel. } \\
\text { Correct orientation of the insert was analyzed } \\
\text { by sequencing. }\end{array}$} \\
\hline P 793 & $\begin{array}{l}\text { pEF-HA-Nup358 } \\
\text { aa } 1-2448\end{array}$ & $\begin{array}{l}628 \\
630\end{array}$ & \multicolumn{2}{|c|}{$\begin{array}{l}\text { The coding DNA for the Nup358 fragment aa } \\
852-2448 \text { was amplified by PCR, and digested } \\
\text { by EcoRV and NotI. It was cloned into pEF-HA- } \\
\text { Nup358 aa 1-1133 (P 732) which was digested } \\
\text { by EcoRV (nct 2556, corresponding to aa 852) } \\
\text { and Notl (MCS). }\end{array}$} \\
\hline P 794 & $\begin{array}{l}\text { pEF-HA-Nup358 } \\
\text { aa } 1-2148\end{array}$ & $\begin{array}{l}628 \\
629\end{array}$ & $\begin{array}{l}\text { Nup358 (P 764) } \\
\text { pEF-HA-Nup358 } \\
\text { aa 1-1133 (P 732) }\end{array}$ & $\begin{array}{l}\text { EcoRV } \\
\text { Notl } \\
\text { see above (P 793) }\end{array}$ \\
\hline P795 & $\begin{array}{l}\text { pEF-HA-Nup358 } \\
\text { aa } 1-1810\end{array}$ & $\begin{array}{l}628 \\
670\end{array}$ & $\begin{array}{l}\text { Nup358 (P 764) } \\
\text { pEF-HA-Nup358 } \\
\text { aa 1-1133 (P 732) }\end{array}$ & $\begin{array}{l}\text { EcoRV } \\
\text { Notl } \\
\text { see above (P 793) }\end{array}$ \\
\hline P 796 & $\begin{array}{l}\text { pEF-HA-Nup358 } \\
\text { aa } 1-1306\end{array}$ & $\begin{array}{l}628 \\
684\end{array}$ & $\begin{array}{l}\text { Nup358 (P 764) } \\
\text { pEF-HA-Nup358 } \\
\text { aa 1-1133 (P 732) }\end{array}$ & $\begin{array}{l}\text { EcoRV } \\
\text { Notl } \\
\text { see above (P793) }\end{array}$ \\
\hline P797 & $\begin{array}{l}\text { pEF-HA-Nup358 } \\
\text { aа } 1-1170\end{array}$ & $\begin{array}{l}628 \\
684\end{array}$ & $\begin{array}{l}\text { Nup358 (P 764) } \\
\text { pEF-HA-Nup358 } \\
\text { aa 1-1133 (P 732) }\end{array}$ & $\begin{array}{l}\text { EcoRV } \\
\text { Notl } \\
\text { see above (P 793) }\end{array}$ \\
\hline \multirow{3}{*}{ P 798} & \multirow{3}{*}{$\begin{array}{l}\text { pEF-HA-Nup358 } \\
\text { aa 1-1306 F1141A }\end{array}$} & & $\begin{array}{l}\text { Nup358 aa 1-1306 } \\
\text { (P 796) } \\
\text { pcDNA } 3.1\end{array}$ & $\begin{array}{l}\text { EcoRV } \\
\text { Notl }\end{array}$ \\
\hline & & $\begin{array}{l}691 \\
692\end{array}$ & $\begin{array}{l}\text { pcDNA Nup358 } \\
\text { aa } 852-1306\end{array}$ & mutagenesis \\
\hline & & & $\begin{array}{l}\text { pcDNA Nup358 } \\
\text { aa 852-1306 F1141A } \\
\text { pEF-HA-Nup358 } \\
\text { aa 1-1306 (P 796) }\end{array}$ & $\begin{array}{l}\text { EcoRV } \\
\text { Notl }\end{array}$ \\
\hline P 799 & $\begin{array}{l}\text { pEF-HA-Nup358 } \\
\text { aa } 806-1000\end{array}$ & $\begin{array}{l}734 \\
706\end{array}$ & $\begin{array}{l}\text { Nup358 (P 796) } \\
\text { pEF-HA_MCS2 }\end{array}$ & $\begin{array}{l}\text { Ncol } \\
\text { Spel }\end{array}$ \\
\hline
\end{tabular}




\begin{tabular}{|l|l|l|l|l|}
\hline$\#$ & Name & $\begin{array}{l}\# \\
\text { Oligo }\end{array}$ & $\begin{array}{l}\text { Template } \\
\text { Vector }\end{array}$ & $\begin{array}{l}\text { Restriction sites/ } \\
\text { description }\end{array}$ \\
\hline P 800 & $\begin{array}{l}\text { pEF-HA-Nup358 } \\
\text { aa 806-1170 }\end{array}$ & & $\begin{array}{l}\text { EcoRV (nct 2556) and Spel (MCS), the } \\
\text { fragment was cloned into pEF-HA-Nup358 } \\
\text { aa 806-1133 (P 601) , which was digested by } \\
\text { EcoRV (nct 2556) and Spel (MCS) }\end{array}$ \\
\hline P 801 & $\begin{array}{l}\text { pEF-HA-Nup358 } \\
\text { aa 806-1306 }\end{array}$ & & $\begin{array}{l}\text { Nup358 aa 1-1306 } \\
\text { (P 796) } \\
\text { Nup358 aa 806-1133 } \\
\text { (P 601) }\end{array}$ & $\begin{array}{l}\text { Spe I } \\
\text { see above (P 793) }\end{array}$ \\
\hline P 802 & $\begin{array}{l}\text { pEF-HA-Syne 1B aa } \\
\text { 1639-1989 } \\
\text { (pEF-HA-Syne 1B) }\end{array}$ & 696 & $\begin{array}{l}\text { Syne 1B aa 1639-1989 } \\
\text { (P 762) } \\
\text { pEF-HA-MCS2 }\end{array}$ & Ncol \\
\hline P 803 & $\begin{array}{l}\text { pEF-HA-Syne 1B- } \\
\text { Nup358 aa 806-1306 }\end{array}$ & $\begin{array}{l}720 \\
721\end{array}$ & $\begin{array}{l}\text { Nup358 aa 806-1306 } \\
\text { (P 801) } \\
\text { pEF-HA-Syne 1B }\end{array}$ & Notl \\
\hline P 804 & pmRFP-Nup358 & $\begin{array}{l}\text { Nup358* (P 790) } \\
\text { pmRFP_MCS }\end{array}$ & $\begin{array}{l}\text { Notl } \\
\text { Xhol }\end{array}$ \\
\hline
\end{tabular}




\subsubsection{Antibodies}

The following antibodies were used for protein detection in western blot (WB) and immunofluorescence (IF):

\section{Primary antibodies}

\begin{tabular}{|c|c|c|c|c|}
\hline Antibody & Immunogen & Organism & Origin/Company & Dilution \\
\hline$\alpha-G F P$ & GFP aa 1-238 & rabbit & Santa Cruz & $\begin{array}{l}\text { IF 1:500 } \\
\text { WB 1:500 }\end{array}$ \\
\hline$\alpha-H A$ & $\begin{array}{l}\text { epitope } \\
\text { CYPYDVPDYASL }\end{array}$ & mouse & Covance & $\begin{array}{l}\text { IF 1:1,000 } \\
\text { WB 1:1,000 }\end{array}$ \\
\hline $\begin{array}{l}\alpha-H A \\
\text { clone } 12 \text { CA5 }\end{array}$ & $\begin{array}{l}\text { X47 HA1 aa } 76-111 \\
\text { epitope YPYDVPDYA }\end{array}$ & mouse & (Niman et al., 1983) & $\begin{array}{l}\text { WB 1:1,000 } \\
\text { preferred for } \\
\text { IP }\end{array}$ \\
\hline$\alpha$-His & His-tag & mouse & Novagen & WB 1:1,000 \\
\hline$\alpha$-importin $\alpha$ & $\begin{array}{l}\text { Rchl } \\
\text { (human isoform 2) }\end{array}$ & rabbit & Dr. D. Görlich ${ }^{*}$ & WB 1:1,000 \\
\hline $\begin{array}{l}\alpha \text {-importin } \beta \\
G, H\end{array}$ & importin $\beta$ & rabbit & Kehlenbach lab & $\begin{array}{l}\text { IF 1:500 } \\
\text { WB 1:500 }\end{array}$ \\
\hline$\alpha$-myc 9 E10 & myc-tag & mouse & Santa Cruz & IF 1:200 \\
\hline $\begin{array}{l}\alpha-N u p 214 \text { 'F' } \\
\text { aff. pur. }\end{array}$ & $\begin{array}{l}\text { human Nup214 } \\
\text { C-terminus }\end{array}$ & rabbit & Kehlenbach lab & IF 1:200 \\
\hline $\begin{array}{l}\alpha \text {-Nup358 } \\
\text { aff. pur }\end{array}$ & $\begin{array}{l}\text { human Nup358 } \\
\text { aa } 2553-2838\end{array}$ & goat & (Hutten et al., 2008) & $\begin{array}{l}\text { IF } 1: 1,000 \\
\text { WB 1:2,000 }\end{array}$ \\
\hline $\begin{array}{l}\alpha-N u p 358 \\
\text { aff. pur }\end{array}$ & $\begin{array}{l}\text { human Nup358 } \\
\text { aa } 3062-3223\end{array}$ & rabbit & Melchior Lab ${ }^{\dagger}$ & IF 1:500 \\
\hline $\begin{array}{l}\alpha-\operatorname{RanGAP1} \\
\text { aff. pur }\end{array}$ & murine RanGAP1 & goat & $\begin{array}{l}\text { Melchior Lab } \\
\text { (Pichler et al., 2002) }\end{array}$ & $\begin{array}{l}\text { IF } 1: 2,000 \\
\text { WB } 1: 2,000\end{array}$ \\
\hline$\alpha$-transportin & $\begin{array}{l}\text { human transportin } \\
\text { N-terminus }\end{array}$ & mouse & BD Bioscience & WB 1:1,000 \\
\hline
\end{tabular}

\footnotetext{
* Prof. Dr. Dirk Görlich, Max Planck Institute for Biophysical Chemistry, Goettingen

+ Melchior Lab, ZMBH, University of Heidelberg
} 


\section{Secondary antibodies}

The secondary antibodies in this work were raised in donkey against the constant region of goat, mouse and rabbit immunoglobulines and were cross-absorbed against other species.

Horseradish peroxidase-conjugated (HRP) secondary antibodies for western blot analysis were obtained from Dianova, and were used at a dilution of 1:5,000.

Secondary antibodies for immunofluorescence conjugated to Alexa488, Alexa594, Alexa633 and Alexa647 were obtained from Molecular Probes and were used at a dilution of 1:1,000. 


\subsubsection{Proteins}

Proteins declared as common lab stocks were purified by members of the Kehlenbach lab, and are available as common protein stocks in the lab.

\begin{tabular}{ll} 
Protein & Source \\
\hline GST-DDX43 & this work \\
GST-DDX59 & this work \\
GST-Sox 6 & this work \\
His-exportin 4 & Thomas Güttler ${ }^{*},($ Lipowsky et al., 2000) \\
His-importin $\alpha$ & common lab stock, (Hu et al., 1996) \\
His-S-importin $\beta$ & common lab stock, (Chi et al., 1997) \\
His-transportin & common lab stock, (Baake et al., 2001) \\
His-importin 5 & common lab stock, (Jäkel et al., 1998) \\
His-importin 7 & common lab stock, (Wohlwend et al., 2007) \\
His-importin 9 & common lab stock, (Mühlhäusser et al., 2001) \\
His-importin 13 & common lab stock, (Mingot et al., 2001) \\
RanQ69L & common lab stock, (Melchior et al., 1995b) \\
Ran wt & common lab stock, (Melchior et al., 1995b)
\end{tabular}

Loading of $2 \mu \mathrm{M}$ Ran with $5 \mathrm{mM}$ GTP in presence of $15 \mathrm{mM}$ EDTA and $40 \mathrm{mM} \mathrm{MgCl}_{2}$ is described elsewhere (Kehlenbach et al., 1999).

\footnotetext{
* Thomas Güttler, Görlich Lab, Max Planck Institute for Biophysical Chemistry, Goettingen
} 


\subsection{Molecular biology methods}

\subsubsection{Plasmid DNA purification}

Small amounts of plasmid DNA were prepared by alkaline lysis (Birnboim et al., 1979) and subsequent precipitation. $2 \mathrm{ml}$ overnight cultures of E.coli $\mathrm{DH} 5 \alpha$ in the stationary phase (in case of the long HA-Nup 358 fragments $8 \mathrm{ml}$ culture) were harvested by centrifugation for $2 \mathrm{~min}$ at $5,000 \mathrm{rpm}$ at room temperature and completely resuspended in $200 \mu$ r resuspension buffer P1 (see Material 2.1.4 for buffer compositions). After mixing with $200 \mu$ l alkaline lysis buffer P2, the lysate was incubated for $5 \mathrm{~min}$ at room temperature before proteins and chromosomal DNA were precipitated by the addition of $200 \mu$ neutralization buffer P3. The lysate was cleared from insoluble components by centrifugation for $10 \mathrm{~min}$ with $13,000 \mathrm{rpm}$ at room temperature. The plasmid DNA was precipitated by adding $420 \mu \mathrm{l}$ of 2-propanol to $600 \mu \mathrm{l}$ of the supernatant. After centrifugation for $30 \mathrm{~min}$ with 13,000 rpm at room temperature, the pellet was washed with $70 \%(v / v)$ ethanol, dried and resuspended in $30 \mu \mathrm{l} \mathrm{TE-buffer} \mathrm{(pH} \mathrm{7.4).}$

High quality isolation of DNA was necessary for plasmids used for transfection of cultured cells. Purification of middle or large quantities of DNA was performed using NucleoBond ${ }^{\circledR}$ PC 100 or PC 500 Kit from Macherey \& Nagel, according to the manufacturer's instructions.

\subsubsection{Measurement of DNA concentration and purity}

DNA concentration of DNA was quantified by measuring the OD at $260 \mathrm{~nm}$; an extinction of 1 corresponded to $\sim 50 \mu \mathrm{g} / \mathrm{ml}$ (Sambrook et al., 1989). The ratio of $\mathrm{OD}_{260}$ to $\mathrm{OD}_{280}$, ideally 1.8 , revealed information about the purity of the DNA. The concentration of the plasmid or vector was set to $1 \mu \mathrm{g} / \mu \mathrm{l}$. 


\subsubsection{Polymerase chain reaction (PCR)}

The principle of the polymerase chain reactions (in the following PCR) traces back to Mullis (Mullis, 1990). Amplification of specific DNA fragments was performed by PCR with two primers defining beginning and end of the amplifying DNA. The reactions were set up in a final volume of $50 \mu \mathrm{l}$ using 10-500 ng of template DNA (Phusion or Vent polymerase, respectively), 300-500 $\mathrm{nM}$ of each, forward and reverse primer, $200 \mu \mathrm{M}$ dNTPs and 1-2 $U$ polymerase. The annealing temperature was calculated for the annealing part of the primers according to online calculators ${ }^{*}$. As a basic rule for the Phusion polymerase, annealing of primers $>20$ nct was performed for $10-30 \mathrm{~s}$ at a temperature $3^{\circ} \mathrm{C}$ above the lower melting temperature of the two primers. Annealing of primers $\leq 20$ nct was done at a temperature equal to the one of the lower primer. The amplification time was chosen according to the given processivity of the polymerase. The cycler was programmed according to table 1 .

\begin{tabular}{l|llll} 
Cycle step & Phusion & \multicolumn{3}{c}{ Vent } \\
& Temperature $\left[{ }^{\circ} \mathrm{C}\right]$ & Time $[\mathbf{s}]$ & Temperature $\left[{ }^{\circ} \mathbf{C}\right]$ & Time $[\mathbf{s}]$ \\
\hline Denaturation & 98 & 30 & 96 & 120 \\
Denaturation & 98 & $5-10$ & 96 & 30 \\
Annealing & $45-72$ & $10-30$ & $54-60$ & 60 \\
Extension & 72 & $15-30 / 1 \mathrm{~kb}$ & 72 & $60 / 500 \mathrm{bp}$ \\
Extension & 72 & $10 \mathrm{~min}$ & 72 & $10 \mathrm{~min}$ \\
Cooling & 4 & $\infty$ & 4 & $\infty$
\end{tabular}

Table 1: PCR-program for Phusion and Vent polymerase: the steps in the shaded box were repeated 25-30 times.

\footnotetext{
*Phusion: https://www.finnzymes.fi/tm_determination.html

Other polymerases: http://www.basic.northwestern.edu/biotools/oligocalc.html
} 


\subsubsection{Agarose gel electrophoresis}

Agarose gel electrophoresis is used to separate DNA by size in order to analyze or identify PCR products and to purify digested DNA fragments. Depending on the size of the expected DNA agarose gels from 0.8 to $1.5 \%(\mathrm{w} / \mathrm{v})$ in TAE buffer were prepared. To make the DNA visible $5 \mu \mathrm{g} / \mathrm{ml}$ of the fluorescent and intercalating agent ethidium bromide (Sharp et al., 1973) was added to the gel. The respective DNA samples were supplemented with an appropriate amount of 10x DNA loading dye and loaded onto the agarose gel. $4 \mu \mathrm{l}$ of the $1 \mathrm{~kb}$ DNA ladder was run in parallel. Gel electrophoresis was performed at 75-120 mA at room temperature. Bands were visualized with UV light (wavelength $365 \mathrm{~nm}$ ).

\section{Isolation of DNA from agarose gels}

After separation of DNA fragments by agarose gel electrophoresis, bands were cut out of the gel. Afterwards, DNA fragments were extracted using the NucleoSpin ${ }^{\circledR}$ Extract II Kit from Macherey \& Nagel according to the manufacturer's instructions. DNA was eluted with $30 \mu \mathrm{l}$ (insert) or $50 \mu \mathrm{l}$ (vector) elution buffer.

\subsubsection{Restriction of DNA by endonucleases}

Restriction enzymes cut double stranded DNA at specific recognition nucleotide motifs, also known as restriction sites. According to the manufacturers' suggestions, the DNA was incubated with the appropriate amount of the enzymes, ranking from 2-10 $U$, in parallel and the respective buffer at the optimal temperature, mostly $37^{\circ} \mathrm{C}$. For a preparative digestion, 4-6 $\mu \mathrm{g}$ of DNA were used in a total volume of $30 \mu \mathrm{l}$. Much less DNA (0.5-2 $\mu \mathrm{g})$ was used for an analytic digestion. The reaction was usually incubated for $2-3 \mathrm{hr}$ at $37^{\circ} \mathrm{C}$. 


\section{Dephosphorylation of digested plasmid DNA}

To prevent self-ligation of digested and linearized vectors, alkaline phosphatase from calf intestine (calf intestinal phosphatase, CIP) could be used to remove the 5' phosphates from the nucleic acid.

The DNA was incubated with $1 \mathrm{U}$ CIP for $15 \mathrm{~min}$ at $37^{\circ} \mathrm{C}$ after the restriction reaction. To achieve inactivation of the alkaline phosphatase, the enzyme was either heat-inactivated for $10 \mathrm{~min}$ at $65^{\circ} \mathrm{C}$ or stopped by the addition of DNA loading dye for gel electrophoresis.

\subsubsection{Ligation of DNA fragments}

Ligations were set up at a molar ratio of 3:1 - 6:1 of insert to vector. The $10 \mu$ reaction set-up contained 2.5 U T4 DNA ligase and some ATP in addition to the supplied ligation buffer. To mix the appropriate amount of DNA, its concentration was estimated in a quantitative agarose gel. The ligation was performed either for 1 hour at $25^{\circ} \mathrm{C}$ or overnight at $16^{\circ} \mathrm{C}$. Especially for blunt-end ligations, $1 \%$ PEG 4000 was added. The reaction was heat-inactivated for $10 \mathrm{~min}$ at $65^{\circ} \mathrm{C}$, and then completely transformed into $\mathrm{DH} 5 \alpha$.

\subsubsection{Oligonucleotide cloning}

To introduce additional restriction sites or localization signals, oligonucleotides were designed with an appropriate overhang to match the restriction sites of the target vector. To anneal the oligonucleotides, they were combined at a concentration of $2 \mu \mathrm{M}$ each in $100 \mu \mathrm{l} 2 \mathrm{x}$ annealing buffer. The reaction was heated for $3 \mathrm{~min}$ at $95^{\circ} \mathrm{C}$. Afterwards, the mixture was set to room temperature for one hour. To ligate the annealed oligonucleotide pair with the predigested vector, it needed to be phosphorylated. Therefore, the designed pair of oligonucleotides was incubated with $10 \mathrm{U}$ T4 polynucleotide kinase and $20 \mu \mathrm{m}$ ATP for $30 \mathrm{~min}$ at $37^{\circ} \mathrm{C}$. The reaction was 
stopped by incubation for $10 \mathrm{~min}$ at $65^{\circ} \mathrm{C}$. Approximately $50 \mathrm{nM}$ of the annealed oligonucleotide was used in a standard ligation reaction with $40 \mathrm{ng}$ digested vector.

\subsubsection{Sequencing of DNA}

All plasmids constructed via PCR amplification were verified by DNA sequencing based on the chain-terminating method established by Sanger, Nicklen and Coulsen (Sanger et al., 1977). The sequencing reactions were performed with BigDye Terminator v1.1 cycle sequencing kit, and contained 0.2-1 $\mu$ g plasmid DNA, $1 \mu \mathrm{m}$ primer, $2 \mu \mathrm{l}$ sequencing buffer and $1 \mu \mathrm{l}$ sequencing mix in a final volume of $10 \mu \mathrm{l}$. PCR was performed according to the program in table 2 . After stopping the reaction with $1 \mu \mathrm{l}$ of each $3 \mathrm{M} \mathrm{NaOAc}(\mathrm{pH} 5.2)$ and 125 mM EDTA, the PCR product was precipitated by addition of $50 \mu$ ice-cold absolute ethanol and centrifugation for $15 \mathrm{~min}$ at 13,000 rpm. The pellet was washed with $70 \%(\mathrm{v} / \mathrm{v})$ ethanol, dried and resuspended in $15 \mu \mathrm{l}$ formamide (Hi-dye). The samples were analyzed in a Genetic Analyzer 3100 (Applied Biosystems) at the Goettingen Center for Molecular Biosciences (GZMB*). The sequences were analyzed using a free of charge version of the software Vector NTI (Invitrogen).

\begin{tabular}{c|cc} 
Cycle step & Temperature $\left[{ }^{\circ} \mathbf{C}\right]$ & Time $[\mathbf{s}]$ \\
\hline Initial denaturation & 96 & 120 \\
Denaturation & 96 & 10 \\
Annealing & 55 & 15 \\
Extension & 60 & 240 \\
Cooling & 4 & $\infty$
\end{tabular}

Table 2: PCR-program for sequencing of DNA: the steps in the shaded box were repeated 25 times.

\footnotetext{
${ }^{*}$ GZMB, Georg-August University, Goettingen
} 


\subsubsection{Site-directed mutagenesis}

Nucleotide exchanges were generated using the quick change site-directed mutagenesis kit protocol (Stratagene). For that purpose, two oligonucleotides with the appropriate mutation and complementarily to each other were generated, and a PCR reaction was used to introduce these site-specific mutations in the double stranded template DNA. Typically, a reaction mixture contained $500 \mathrm{ng}$ of template DNA, $250 \mathrm{nM}$ of complementary oligonucleotides, $200 \mu \mathrm{m}$ dNTPs, 10x thermo buffer and $2 \mathrm{U}$ Vent polymerase in a total volume of $50 \mu \mathrm{l}$. The mixture was split into five aliquots. One was incubated at room temperature, representing the negative control for the efficiency of Dpnl digestion. The remaining four aliquots were used to perform the PCR according to following program applying a temperature gradient for annealing (table 3).

After the PCR reaction, $5 \mathrm{U}$ of the restriction enzyme Dpnl and the corresponding buffer was added to all samples and incubated for $2 \mathrm{hr}$ at $37^{\circ} \mathrm{C}$. Dpnl cleaves specifically methylated DNA, and therefore leads to digestion of the methylated maternal DNA, whereas the newly synthesized DNA carrying the mutation is unmethylated and thus not cleaved by the enzyme. The complete reaction was transformed into $E$. coli $\mathrm{DH} 5 \alpha$. The resulting clones were analyzed by DNA sequencing.

\begin{tabular}{c|cc} 
Cycle step & Temperature $\left[{ }^{\circ} \mathbf{C}\right]$ & Time $[\mathbf{s}]$ \\
\hline Initial denaturation & 95 & 120 \\
Denaturation & 95 & 30 \\
Annealing & gradient $54-64$ & 15 \\
Extension & 72 & $60 / 500 \mathrm{bp}$ \\
Final extension & 72 & $10 \mathrm{~min}$ \\
Cooling & 4 & $\infty$
\end{tabular}

Table 3: PCR-program for mutagenesis: the steps in the shaded box were repeated 25-30 times. 


\subsubsection{Prokaryotic Cells}

\section{Cultivation of $E$. coli}

Bacteria were kept in LB-medium at $37^{\circ} \mathrm{C}$ for standard cultures, supplemented with the required antibiotics: ampicillin $100 \mu \mathrm{g} / \mathrm{ml}$, kanamycin $60 \mu \mathrm{g} / \mathrm{ml}$, chloramphenicol $30 \mu \mathrm{g} / \mathrm{ml}$ for liquid cultures, half the concentration was used for LB agar plates. Liquid cultures were shaken at $160 \mathrm{rpm}$.

\section{Preparation of chemical competent bacteria}

Chemical competent E.coli were prepared from a $200 \mathrm{ml}$ growing culture with an optical density OD600 of 0.5 . After $10 \mathrm{~min}$ incubation on ice, bacteria were harvested by centrifugation at $5,000 \mathrm{x}$ at $4^{\circ} \mathrm{C}$. The cell pellet was resuspended in $200 \mathrm{ml}$ ice-cold TFB-I-buffer and incubated on ice for $2 \mathrm{hr}$. The cells were again collected with 5,000x $\mathrm{g}$ at $4^{\circ} \mathrm{C}$ and resuspended in $8 \mathrm{ml}$ of sterile cold TFB-II-buffer. Aliquots of $100 \mu \mathrm{l}$ were frozen in liquid nitrogen and stored at $-80^{\circ} \mathrm{C}$.

\section{Transformation of competent bacteria}

Chemical competent E.coli DH5 $\alpha$ were thawed on ice, and incubated with the respective amount of DNA for 20 min on ice. Incorporation of DNA was achieved by heat-shock at $42^{\circ} \mathrm{C}$ for $90 \mathrm{~s}$, followed by incubation on ice for $1 \mathrm{~min}$. For regeneration, bacteria were supplemented with $400 \mu \mathrm{l}$ ice-cold SOC medium and incubated at $37^{\circ} \mathrm{C}$ for 1 hour. Finally, the transformed cells were plated on LB agar plates supplemented with the respective antibiotic for the transformed plasmid. 


\subsection{Biochemical methods}

\subsubsection{Separation and detection of proteins}

\section{SDS polyacrylamide gel electrophoresis (SDS PAGE)}

Separation of proteins was performed by SDS polyacrylamide gel electrophoresis (SDS-PAGE) according to the system described by Laemmli (Laemmli, 1970). It was performed in a discontinuous buffer system including two layers of gel, namely stacking and resolving gel.

In this work, small gels with concentrations between 7-12\% were used. Proteins dissolved in SDS-PAGE loading buffer (1x final concentration) were heated at $95^{\circ} \mathrm{C}$ for $5 \mathrm{~min}$. In addition to the samples, a protein ladder was loaded onto the gel to estimate the molecular weight of the resolved proteins. In case of Nup358 and its deletions, the protein ladder SeeBlue2 was additionally loaded to the unstained protein ladder. The gels were run with Laemmli running buffer with an electric current of $20 \mathrm{~mA}$ applied for migration.

\section{Staining Methods}

Coomassie stain

Proteins can be directly stained with the dye coomassie brilliant G-250 by unspecific interactions with cationic amino acids. After separation of the proteins in SDS-PAGE, the gel is fixed at least 15 minutes in coomassie fixing solution, followed by staining with the coomassie staining solution for 2 minutes. Excess of the dye is removed by several washing steps with $\mathrm{H}_{2} \mathrm{O}$.

\section{Silver stain}

Detection of small amounts of proteins in gels was achieved by silver staining, a method developed by Merril and coworkers (Merril et al., 1981). After separation of the proteins by SDS-PAGE, the gel was fixed for at least $1.5 \mathrm{hr}$ in silver gel fixing solution, washed 
3 times for $20 \mathrm{~min}$ in $50 \%(\mathrm{v} / \mathrm{v})$ ethanol, incubated in fresh $0.01 \%(\mathrm{w} / \mathrm{v}) \mathrm{Na}_{2} \mathrm{~S}_{2} \mathrm{O}_{3}$ for $1 \mathrm{~min}$, washed 3 times for $20 \mathrm{~s}$ in water and stained for $20 \mathrm{~min}$ in fresh $0.1 \%(\mathrm{w} / \mathrm{v}) \mathrm{AgNO}$. The stained gels were developed by incubation in silver gel developing solution. When the protein bands became visible, the gel was washed with water and the reaction was stopped with $10 \mathrm{mM}$ EDTA.

Colloidal coomassie stain

Another very sensitive dye to stain proteins is colloidal coomassie brilliant G-250 (Neuhoff et al., 1988, modified by D. Hesse, Martinsried). The colloidal coomassie dye was prepared at least $24 \mathrm{hr}$ before use. The working solution was freshly prepared with $80 \%(\mathrm{v} / \mathrm{v})$ dye stock and $20 \%(\mathrm{v} / \mathrm{v})$ methanol. The gel was fixed with $40 \%(\mathrm{v} / \mathrm{v}) \mathrm{EtOH}$ and $10 \%(\mathrm{v} / \mathrm{v})$ acetic acid for at least $60 \mathrm{~min}$, and afterwards washed twice with water for $10 \mathrm{~min}$. After incubation with the dye solution overnight, excess of the dye was removed by washing several times with $1 \%(\mathrm{v} / \mathrm{v})$ acetic acid.

\subsubsection{Protein transfer via western blot and immunological detection of proteins}

To detect proteins via specific antibodies after SDS-PAGE, they have to be transferred from the gel onto a nitrocellulose membrane (Towbin et al., 1979). Western blotting was performed using the wet blot or tank blot method which is, especially for big proteins, very efficient. This was performed by placing the gel onto the nitrocellulose membrane, sandwiched by two Whatman papers on each side. Everything was equilibrated with western blot transfer buffer. Thereafter, the blotting cassette was put into the blot tank. The proteins were transferred vertically to a nitrocellulose membrane for $75 \mathrm{~min}$ at constant $400 \mathrm{~mA}$. Blotting was performed at $4^{\circ} \mathrm{C}$ under permanent stirring to avoid warming up of the system. To check uniform blotting, the proteins were stained with Ponceau S solution afterwards. Excess of the dye was washed away with $1 \%(\mathrm{v} / \mathrm{v})$ acetic acid. 
After the proteins had been transferred to the nitrocellulose membrane, they were probed with specific primary antibodies. An HRP-coupled, secondary antibody was directed against the primary antibody which allowed detection of the blotted proteins.

As a first step, unspecific binding sites on the membrane were blocked by incubation with blocking buffer ( $5 \%(\mathrm{w} / \mathrm{v})$ skim milk in PBS-Tween) for $10 \mathrm{~min}$ at room temperature. The membrane was incubated with the primary antibody in an appropriate dilution overnight at $4^{\circ} \mathrm{C}$, and then washed 3 times for 5 min with PBS-Tween. The incubation with the secondary antibody in a dilution of 1:5,000 took place for $30 \mathrm{~min}$ at room temperature. After removing unbound antibody by washing with PBS-Tween, bound antibody was detected by chemiluminescence using an ECL kit from Millipore. Exposure times of the films were chosen according to the strength of the signal. Films were developed using either an automatic developing machine or a chemiluminescence imaging system (LAS 3,000).

In case a single membrane was used to detect several proteins consecutively, old signals were quenched by drying the membrane, putting it at $-20^{\circ} \mathrm{C}$ for $20 \mathrm{~min}$, followed by incubation with Ponceau $S$ and blocking buffer. This procedure inactivated the old peroxidase. The order of incubation was chosen according to the expected strength of the signal (starting with weak signals) and the size of the protein (starting with lower molecular weight proteins). Alternatively, membranes were cut to separate specific molecular weight ranges.

\subsubsection{Expression and purification of recombinant GST-tagged proteins}

All GST-proteins used in this work were prepared as followed: the corresponding construct in a pGEX-vector was expressed in E. coli Rosetta with the antibiotics ampicillin and chloramphenicol supplied. 1.5 I LB-medium with both antibiotics was directly inoculated, and the culture was grown till an $\mathrm{OD}_{600}$ of 0.6 was reached. $2 \% \mathrm{EtOH}$, $50 \mathrm{mM} \mathrm{K}_{2} \mathrm{HPO}_{4}$ and $0.1 \mathrm{mM}$ IPTG were added and the temperature was shifted to $18^{\circ} \mathrm{C}$. GST-DDX43 and GST-DDX59 were both induced with 0.25 mM IPTG. Protein expression took place overnight. Afterwards, the cells were centrifuged, and resuspended in 
$40 \mathrm{ml}$ buffer A which contained freshly added $2 \mathrm{mM} \mathrm{DTT}$ and $1 \mu \mathrm{g} / \mathrm{ml}$ of each protease inhibitor aprotinin, leupeptin and pepstatin. Cell lysis was achieved using an emulsi flex. Bacterial debris was removed by centrifugation at $100,000 \mathrm{x} g$ at $4^{\circ} \mathrm{C}$ for $30 \mathrm{~min}$ in a Beckman Type 45 Ti rotor. The supernatant was diluted in $40 \mathrm{ml}$ buffer $B$ to reduce the Triton concentration. $1 \mathrm{ml}$ of glutathione sepharose equilibrated with buffer $B$ and the supernatant were incubated for $2 \mathrm{hr}$ at $4^{\circ} \mathrm{C}$ by slow rotation. Afterwards, the beads were washed 3 times for $5 \mathrm{~min}$ with buffer B. Bound proteins were eluted with $15 \mathrm{mM}$ glutathione $(\mathrm{pH} 8)$ in $1.5 \mathrm{ml}$ fractions. Protein-containing fractions were combined, diluted 1:3 with transport buffer, and concentrated using a vivaspin column, which was blocked with $10 \%(\mathrm{w} / \mathrm{v})$ BSA. A last dilution step of 1:10 followed, and the proteins were concentrated to approximately $0.5-1 \mathrm{ml}$. Protein concentrations were estimated by SDS-PAGE by loading different amounts of the protein and a BSA standard. After the purification, proteins were aliquotted, flash frozen in liquid nitrogen and stored at $-80^{\circ} \mathrm{C}$.

\subsubsection{In vitro binding assays with GST- and His-labeled proteins}

In vitro binding assays were performed with recombinant immobilized GST-labeled proteins and His-tagged import receptors to analyze their interaction. $5 \mu \mathrm{g}$ GST-tagged protein was immobilized on $15 \mu$ glutathione sepharose, which was equilibrated with buffer B containing $1 \%(\mathrm{w} / \mathrm{v})$ BSA, 2 mM DTT and protease inhibitors. After incubation for $2 \mathrm{hr}, 5 \mu \mathrm{g}$ of import receptor was added and binding could occur within $2 \mathrm{hr}$ at $4^{\circ} \mathrm{C}$. The

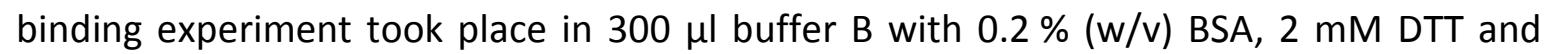
protease inhibitors. Where indicated, reactions contained $2 \mu \mathrm{M}$ RanQ69L loaded with GTP as described (Kehlenbach et al., 1999). After 3 times washing with buffer B, bound proteins were eluted with $50 \mu \mathrm{l} 2 \mathrm{x}$ SDS loading buffer and subjected to SDS-PAGE followed by either silver staining or colloidal coomassie staining or western blotting. 


\subsection{Cell biology methods for mammalian cell culture}

\subsubsection{Cultivation of adherent cells}

Adherent HeLa P4 and 293T HEK cells were cultured in Dulbecco's modified Eagle Medium (DMEM) supplemented with $10 \%(\mathrm{v} / \mathrm{v})$ fetal calf serum (FCS) and $2 \mathrm{mM}$ glutamine. Cells were maintained in a humidified incubator with $5 \%(\mathrm{v} / \mathrm{v}) \mathrm{CO}_{2}$ at $37^{\circ} \mathrm{C}$. In general, cells were split at a $1 / 10$ ratio just before reaching confluence. For this purpose, cells were washed with sterile PBS, detached from the culture dishes with trypsin/EDTA, and diluted with fresh medium.

\subsubsection{RNA interference (RNAi) experiments}

RNA interference (RNAi) is a very specific and efficient method to suppress the expression of certain proteins on the post-transcriptional level (Tuschl et al., 1999; Tuschl, 2001). In this work, RNAi knockdown experiments were performed to suppress the translation of Nup358 by transfection of short interfering RNAs (siRNA) directed against the protein's mRNA into cells using the reagent oligofectamine.

HeLa P4 cells were transfected with validated siRNA oligonucleotides directed against the mRNA of Nup358 according to an established protocol (Hutten et al., 2006). In short, siRNA was obtained from Ambion (standard purity) as annealed oligonucleotides. Cells were plated on a 24 well dish and transfected with $100 \mathrm{nM}$ siRNA on day 1 . Two days later cells were split onto Poly-L-Lysine coated cover slips and retransfected with $100 \mathrm{nM}$ siRNA. DNA transfection of the reporter proteins was performed one day later using the protocol for transient transfection. Cells were either fixed or further treated on day 5. During the procedure, no antibiotics were used. Control cells were cultivated in parallel, but were not treated with siRNA or transfection reagent. 


\section{Amount for one 24 well [ $\mu l]$}

Oligofectamine

Optimem added to oligofectamine SiRNA $(20 \mu \mathrm{M})$

Optimem added to siRNA

New medium on cells

\section{1}

9

1.75

38.25

300

Table 4: Pipette-scheme for siRNA transfection

\subsubsection{Coating of cover slips with Poly-L-Lysine}

Cover slips were treated with the positively charged Poly-L-Lysine to improve adherence of the cells to the glass cover slip. After washing with 2-propanol and drying, the cover slips were covered with $0.01 \%(\mathrm{v} / \mathrm{v})$ Poly-L-Lysine and incubated for $30 \mathrm{~min}$ at room temperature. After washing twice with sterile water, the cover slips were completely dried. Incubation with UV light ( $3 \mathrm{~min}$ at $0.12 \mathrm{~J} / \mathrm{cm} 2)$ provides the required sterility for the use in cell culture.

\subsubsection{Transient transfection of DNA}

For localization- and large-scale experiments such as immunoprecipitations, the calcium phosphate method was applied (Ausubel et al., 1994). The appropriate amount of DNA was supplemented with freshly made $200 \mathrm{mM}$ calcium chloride from a $2 \mathrm{M}$ stock (see table 5 for more details). Equal parts of $2 x$ HBS buffer ( $\mathrm{pH} 6.98$ ) was added by bubbling with a glass pipette and vortexing afterwards in order to yield small calcium phosphate/DNA precipitates. The mixture was incubated for $20 \mathrm{~min}$ at room temperature and then added to the cells which were $15-20 \%$ confluent. 6-24 hr after transfection, the precipitates were washed away with PBS, and new medium was added. 
In the case of the HA-Nup358 full-length and deletion mutants, cells were treated with $10 \%$ DMSO in DMEM for $2 \mathrm{~min}$, and washed twice with PBS. This step increased the ability of cells to take up large DNA molecules.

In cotransfection experiments, the ratio of each DNA was adjusted to the expression level of the constructs. For example in the rescue experiments for Nup358 knockdown, 1/7 of the substrate DNA and 7/8 of the Nup358 fragment DNA was used. To reduce expression levels of some GFP-constructs, percentage of their DNA was reduced and filled up with an empty vector, e.g. pEF-HA.

After $20 \mathrm{hr}$ expression time, cells were fixed and processed as described for general detection of fluorescent proteins. Analysis was carried out by fluorescence microscopy.

\begin{tabular}{l|ll} 
& $\mathbf{2 4}$ well $[\boldsymbol{\mu l}]$ & $\mathbf{1 0} \mathbf{~ c m}$ dish $[\boldsymbol{\mu l}]$ \\
\hline $\mathrm{CaCl}_{2}(250 \mathrm{mM})$ & 20 & 500 \\
DNA & $0.8 \mu \mathrm{g}$ & $10-15 \boldsymbol{\mu g}$ \\
$2 x \mathrm{HBS}$ & 20 & 500 \\
Medium cells & 500 & $10 \mathrm{ml}$
\end{tabular}

Table 5: Pipette-scheme for DNA transfection

\subsubsection{In vitro import assays in HeLa cells}

The in vitro import assay is based on the method established by Adam and coworkers (Adam et al., 1990). This assay allows monitoring of nuclear import processes by using recombinant transport receptors in combination with recombinant substrates.

HeLa P4 cells were grown on $12 \mathrm{~mm}$ cover slips to $60 \%$ confluence. Permeabilization was the plasma membrane was performed with $0.015 \%(\mathrm{v} / \mathrm{v})$ digitonin in transport buffer. The buffer was supplemented with $2 \mathrm{mM}$ DTT and $1 \mu \mathrm{g} / \mathrm{ml}$ protease inhibitor. The incubation was done on ice for $5 \mathrm{~min}$. Treatment with digitonin as a non-ionic detergent selectively leads to a permeabilization of the cholesterol-rich plasma membrane while the nuclear envelope stays intact. The endogenous soluble transport receptors were washed 
out of the cells in 3 washing steps for 3 min. Afterwards the permeabilized cells were incubated with $20 \mu \mathrm{l}$ of transport reaction mix at $30^{\circ} \mathrm{C}$ for $30 \mathrm{~min}$. This reaction mix contained $0.5 \mu \mathrm{M}$ transport substrate, $0.5 \mu \mathrm{M}$ recombinant import receptor, $4 \mu \mathrm{m}$ Ran, $2 \%(\mathrm{w} / \mathrm{v}) \mathrm{BSA}$ and an energy-regenerating system consisting of $1 \mathrm{mM}$ ATP, $2.8 \mathrm{mM}$ creatine phosphate and $0.4 \mathrm{U}$ creatine phosphate kinase. For negative controls, the assay was done in the absence of import receptors or at $4^{\circ} \mathrm{C}$. Afterwards, cells were fixed with $3.7 \%(\mathrm{v} / \mathrm{v})$ formaldehyde in PBS for $10 \mathrm{~min}$, and an indirect immunofluorescence procedure followed to detect the substrate.

\subsubsection{The dexamethasone-inducible import system}

Another way to measure nuclear uptake is the dexamethasone-inducible nuclear import system (Love et al., 1998). Here, a NLS fused to the ligand-binding domain of the glucocorticoid receptor (GR) is retained in the cytoplasm, addition of the hormone dexamethasone releases the receptor, and import can take place.

To analyze nuclear import of the dexamethasone-inducible reporters $\mathrm{GR}_{2}-\mathrm{GFP}_{2}-\mathrm{M} 9$ (Hutten et al., 2009) or Rev-GR-GFP (RGG) in vivo, control-treated or siRNA-treated cells were plated on Poly-L-lysine coated cover slips, and transfected with the respective construct. Import was induced by addition of $5 \mu \mathrm{m}$ dexamethasone. After $15 \mathrm{~min}$ at $37^{\circ} \mathrm{C}$ in the incubator, cells were fixed, subjected to indirect immunofluorescence, and analyzed by fluorescence microscopy.

\section{In vivo assay in living cells}

To compare the kinetics of nuclear uptake of control- to siRNA-treated cells, they were plated on Poly-L-lysine coated LabTek ${ }^{\mathrm{TM}}$ chambered cover glass, and transfected with the construct $\mathrm{GR}_{2}-\mathrm{GFP}_{2}-\mathrm{M9}$ (Hutten et al., 2009). Cells were transferred to $\mathrm{CO}_{2}$-independent medium, and nuclear import was induced with $5 \mu \mathrm{M}$ dexamethasone. Images were taken every $10 \mathrm{~s}$ for $15 \mathrm{~min}$ with a ZEISS LSM 510-Meta confocal microscope equipped with an argon laser using the Argon 488 laser line and a 63x Plan-Neofluar 1.3 NA water-corrected 
objective. Laser intensity was set to $2 \%$ to prevent fast bleaching and photo toxic reactions in the living cells. To ensure fast acquisition, images were processed without averaging and with a scan speed of $2.6 \mu \mathrm{s} /$ pixel. The pinhole was set to 2 airy units to collect as much fluorescence as possible without losing confocality. The temperature controlled incubation chamber was equilibrated to $37^{\circ} \mathrm{C}$.

For every time point, nuclear and total cellular fluorescence were measured using the ROI (region of interest) function in the LSM software to get averaged pixel intensities in the designated area. After the background signal was subtracted, the ratio of nuclear/total fluorescence of $\mathrm{GR}_{2}-\mathrm{GFP}_{2}-\mathrm{M} 9$ was plotted against time.

\subsubsection{Immunofluorescence}

Indirect immunofluorescence was performed at room temperature in a dark-wetchamber. HeLa P4 cells grown on $12 \mathrm{~mm}$ glass cover slips were fixed for $10 \mathrm{~min}$ in $3.7 \%(v / v)$ formaldehyde in PBS, and then washed 3 times with PBS. Incubation with $50 \mathrm{mM}$ ammonium chloride in PBS was used for $10 \mathrm{~min}$ to quench free aldehyde groups. After 2 washing steps with PBS, cells were permeabilized for $5 \mathrm{~min}$ with $0.5 \%(\mathrm{v} / \mathrm{v})$ Triton X-100 in PBS. After further washing, cells were blocked with $0.2 \%(\mathrm{w} / \mathrm{v})$ fish gelantine in PBS for $10 \mathrm{~min}$ to avoid unspecific binding of the antibodies. The cover slips were incubated for 50 min with the appropriate first antibody dilution in blocking reagent at room temperature. Between the first and the secondary antibody incubation, the cover slips were washed 3 times for 5 min with PBS. The incubation with the fluorescently labeled secondary antibodies in blocking reagent took $30 \mathrm{~min}$. After washing 3 times for 5 min with PBS, DNA was visualized by incubation with $10 \mu \mathrm{g} / \mathrm{ml}$ Hoechst for $2 \mathrm{~min}$. After 3 more washing steps, the cover slips were dried and then mounted onto microscope slides with fluorescence mounting medium. 


\subsubsection{Fluorescence microscopy}

\section{Confocal microscopy}

Fluorescence was analyzed using an Axiovert $200 \mathrm{M}$ fluorescence microscope with a 63x Plan-Neofluar 1.3 NA water-corrected objective, appropriate filter settings and an x-site series 120 lamp for illumination. Images were taken using a LSM 510-Meta confocal microscope. Excitation was provided by the following laser lines: Diode (405 nm) for Hoechst, Argon458 for CFP, Argon488 for Alexa488 dyes or GFP, Argon514 for YFP, HeNe594 for mRFP, mCherry and Alexa594 dyes and the HeNe633 for the Alexa633/647 dyes. Multi tracking was applied to detect potential cross excitation of the different dyes. The airy unit of the pinhole was set to $1-2$. The signal/noise ratio was decreased by averaging 2-4 images and using an acquisition speed of 1.5-2.5 $\mu \mathrm{s} / \mathrm{pixel}$. To circumvent rapid fading of the fluorescence signal by repeated image acquisition, the laser intensity was adjusted to 8-10 \% for the Diode and the Argon laser, and to $10-20 \%$ for the HeNe lasers depending on the fluorescence signal.

\section{Deconvolution microscopy}

Deconvolution is a computational image processing technique which is utilized for improving the contrast and resolution of digital images captured in the microscope. Hence, a Z-stack consisting of six images were processed with an Olympus IX71 inverted microscope equipped with a 60x oil objective (by courtesy of Prof. Dr. Rehling, Institute of Biochemistry II, University of Goettingen). Deconvolution was performed by the Deltavision software. 


\subsubsection{Quantification of import inhibition in Nup358-depleted cells}

Control or Nup358-depleted cells were transfected with the corresponding cDNA. The cellular localization of the reporter proteins were determined by fluorescence microscopy. The knockdown efficiency of Nup358 was revealed by immunostaining using an $\alpha$-Nup358 antibody. For quantification, cells were grouped into three categories: majority of the reporter protein in the nucleus $(\mathrm{N}>\mathrm{C})$, equal distribution between nucleus and cytoplasm $(\mathrm{N}=\mathrm{C})$ and mainly cytoplasmic localization $(\mathrm{C}>\mathrm{N})$. In general, quantification was performed from at least three independent experiments, each with more than 100 cells showing equal expression levels. Error bars depicted the standard deviation of the mean of three independent experiments. Statistical significance of the data was determined applying a two-tailed and heteroscedastic student's t-test (White, 1980). All calculations and quantifications were performed using Microsoft Office Excel 2007.

In some cases, the analysis of siRNA-treated and control cells revealed considerable variations in the cargo distribution between single experiments. For this reason or for a better comparison between different transfection conditions, the localization in Nup358 knockdown cells overexpressing Nup358 variants was analyzed in relation to the distribution in control cells. The number of control cells in the category $\mathrm{N}>\mathrm{C}$ was normalized to $100 \%$, and the distribution in the siRNA-treated cells was calculated in relation to this.

\section{Data analysis and quantification}

Fluorescence intensities were measured using the ROI function in the LSM software to get averaged pixel intensities in the designated area, and the background signal was subtracted prior to further analysis. The ratio of nuclear/cytoplasmic fluorescence was plotted for the different conditions.

Images were processed using the LSM Image Browser, AxioVision Rel. 4.8 LE and Adobe Photoshop 6.0. 


\subsubsection{Immunoprecipitations}

All buffers for immunoprecipitations contained freshly added $2 \mathrm{mM}$ DTT and $1 \mu \mathrm{g} / \mathrm{ml}$ of each aprotinin, leupeptin and pepstatin. The buffers for the GFP-nanotrap also contained 1 mM PMSF.

\section{Immunoprecipitations using NP40-buffer}

Immunoprecipitations (IP) are used to isolate and concentrate particular proteins from cell lysate containing many thousands of different proteins. Usage of a specific antibody against that certain protein or its tag causes precipitations which can be coupled to solid substrate later on. In this work, immunoprecipitations were performed from 2-4 $10 \mathrm{~cm}$ dish/sample using $1 \mu$ affinity-purified $\alpha$-HA-, $\alpha$-importin $\beta$ or $\alpha$-Nup358 antibody per dish. In case of HA-Nup358 full-length or its truncation mutants, HeLa P4 cells were transfected following the special treatment with $10 \%$ DMSO. Due to low expression, $4 \times 10 \mathrm{~cm}$ dishes were used for one experiment. $48 \mathrm{hr}$ after transfection, 293T HEK cells were washed twice with ice-cold PBS, and $400 \mu$ l cold NP40-buffer supplemented with $2 \%(\mathrm{w} / \mathrm{v})$ BSA was added per dish. Cells were harvested and incubated on ice for $15 \mathrm{~min}$. The lysate was cleared by centrifugation at 100,000x g using the TLA 100.3 rotor for $20 \mathrm{~min}$. Before adding the antibody, a small sample, referred to as input, was taken and supplemented with the appropriate amount of $2 x$ SDS loading buffer. The antibody was incubated with the lysate for $3 \mathrm{hr}$, rotating slowly at $4^{\circ} \mathrm{C}$ before adding 10-15 $\mu$ l Protein A agarose (Roche) per reaction. For the $\alpha$-Nup358 antibody, protein $\mathrm{G}$ agarose was used. The beads had been equilibrated for at least for $3 \mathrm{hr}$ with NP40-buffer containing $10 \%(\mathrm{w} / \mathrm{v})$ BSA. All centrifugation steps with the agarose were performed at $1,000 \mathrm{rpm}$ at $4^{\circ} \mathrm{C}$. The samples were then rotated at $4{ }^{\circ} \mathrm{C}$ for another $1.5 \mathrm{hr}$. The immunoprecipitates were washed 3 times with NP40-buffer. Bound proteins were eluted by either adding $50 \mu \mathrm{l} 2$ x SDS loading buffer or by a two-step elution with the HA-peptide in case of the $\alpha$-HA-antibody.

To this end, synthetic HA-peptide [10 mg/ml] was diluted in NP40-buffer to $400 \mu \mathrm{g} / \mathrm{ml}$, $30 \mu \mathrm{l}$ were added to the agarose and incubated for $10 \mathrm{~min}$ at $30^{\circ} \mathrm{C}$ by gentle shaking. After centrifugation, the supernatant was removed and kept on ice, and another round of 
elution followed. Afterwards, the complete eluate was supplemented with the appropriate amount of 4x SDS sample buffer, and subjected to SDS-PAGE followed by western blotting to check the efficiency of the precipitation and to look for interacting proteins.

\section{Immunoprecipitations of GFP-tagged proteins using the GFP-nanotrap}

Green fluorescent proteins (GFP) and their variants are widely used to study protein localization and dynamics. For biochemical analysis these GFP fusion proteins and their interacting factors can be isolated efficiently via a special immunoprecipitation where a small GFP binding protein of $13 \mathrm{kDa}$ derived from a llama single chain antibody is coupled to NHS sepharose (Rothbauer et al., 2008).

Transfected 293T HEK cells were lysed in $200 \mu$ ice-cold lysis buffer per $10 \mathrm{~cm}$ dish and incubated on ice for $10 \mathrm{~min}$. The cell lysate was then centrifuged for $20 \mathrm{~min}$ at 13,000 rpm at $4^{\circ} \mathrm{C}$. The supernatant was collected carefully, and its volume was adjusted to $500 \mu \mathrm{l}$ with ice-cold dilution buffer. A small sample was taken referred to as input. In between, $15 \mu$ l beads per sample, were equilibrated with dilution buffer by 2 washing steps and centrifugation at $2,700 \mathrm{x}$. Cell lysate was added to the sepharose and incubated for $2-3 \mathrm{hr}$ at $4^{\circ} \mathrm{C}$ while rotating. Afterwards, the beads were washed 3 times with ice-cold wash buffer. In case a binding-assay with recombinant import receptors followed, 2 more washing steps with buffer B, containing $2 \%(w / v)$ BSA, followed. For that, the immunoprecipitated GFP-protein coupled to the beads was transferred to a $500 \mu \mathrm{l}$ eppendorf tube and progressed as described for in vitro binding assays.

In case no binding-assay was performed, the beads were resuspended in $50 \mu \mathrm{l} 2 \times$ SDS sample buffer and boiled at $95^{\circ} \mathrm{C}$. 



\section{$3 \quad$ RESULTS}

\subsection{Nup358 depletion affects nuclear import and not export of a transportin cargo}

Depletion of Nup358 leads to accumulation of specific cargos in the cytoplasm like the human immunodeficiency virus HIV-1 Rev protein (Rev) (Hutten et al., 2008; Hutten et al., 2009) which could result either from impaired import or accelerated export. HIV-1 Rev localizes predominantly to the cytoplasm in Nup358-depleted cells under conditions that specifically block CRM1 activity which points to a role of Nup358 in nuclear import of this protein (Hutten et al., 2009).

Another way to show that import and not export is affected by the depletion of Nup358 by RNA interference (RNAi) is the dexamethasone-inducible nuclear import system (Love et al., 1998) in living cells. Here, the reporter protein $\mathrm{GR}_{2}-\mathrm{GFP}_{2}-\mathrm{M} 9$ (GG-M9) consists of a fusion between two ligand binding domains of the glucocorticoid receptor, a double GFP and the M9-NLS for transportin binding. One advantage of this system is the exact time window of 15 min where nuclear import takes place. After transfection, this protein localized to the cytoplasm in control as well as in Nup358-depleted cells. Treatment with the hormone dexamethasone induced nuclear uptake. Import depended on the M9-NLS as the reporter without this signal sequence did not localize to the nucleus after induction (data not shown). In control cells, the reporter localized predominantly to the nucleus after 15 min (Figure 3-1 A). In contrast, cells with reduced levels of Nup358 displayed a less efficient nuclear import, and the substrate distributed equally between nucleus and cytoplasm (Hutten et al., 2009). Nuclear uptake was very rapid and efficient in control cells as fluorescence in the nucleus compared to total fluorescent increased fourfold (Figure 3-1 B). In Nup358-depleted cells, import took place as well, but the rate was much slower and the nuclear fluorescent increased only twofold.

These observations point towards a role of Nup358 in import rather than in export, for both the importin $\alpha / \beta$ and the transportin pathways. 
A

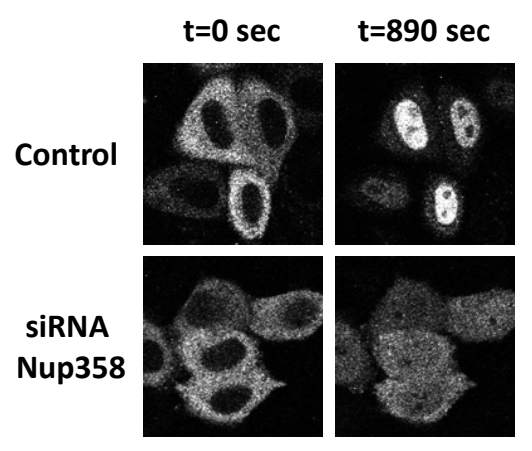

B

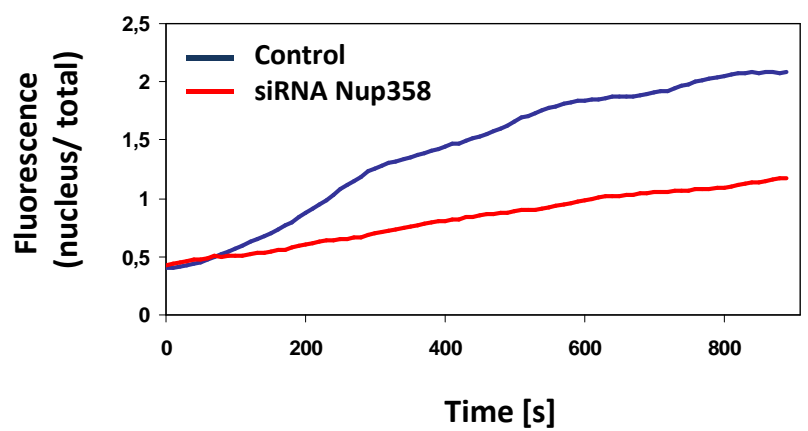

Figure 3-1: Depletion of Nup358 leads to a reduced import kinetic of a transportin-dependent substrate. Control or Nup358-depleted HeLa P4 cells were transfected with a construct coding for $\mathrm{GR}_{2}-\mathrm{GFP}_{2}-\mathrm{M} 9$. After the addition of dexamethasone $(t=0)$, the rate of nuclear import was measured for $15 \mathrm{~min}$. A: Control or siRNA-treated cells before $(t=0 \mathrm{~s})$ and after $(\mathrm{t}=890 \mathrm{~s})$ the addition of dexamethasone are shown. B: The graph shows the increase of the ratio of nuclear to total fluorescence over time. Error bars indicate the standard deviation from the mean of 65 (control) or 74 (Nup358-depleted cells). The experiment was repeated twice with very similar results. Efficient depletion of Nup358 was confirmed in a parallel experiment by immunofluorescence (data not shown). See also Hutten et al., 2009.

\subsection{Nuclear accumulation of a subset of proteins depends on Nup358}

Reduction of Nup358 by RNAi leads to reduced nuclear import of cargos mediated by the importin $\alpha / \beta$ as well as the transportin pathway (Hutten et al., 2008; Hutten et al., 2009). In these studies, artificial reporter proteins with a NES and NLSs specific for the importin $\alpha / \beta$ (cNLS) or transportin pathway (M9) showed significantly reduced nuclear localization in Nup358-depleted cells compared to control cells. Similarly, nuclear import of the HIV-1 Rev protein (Hutten et al., 2009) showed strong dependence on Nup358.

In a screen with 200 nuclear proteins derived from the LIFEdb (database for localization, interaction, functional assays, and expression of proteins, (Bannasch et al., 2004), a number of proteins were identified that localize more cytoplasmically in cells depleted of Nup358 compared to control cells (Hutten, 2007). 
As the LIFEdb contains mostly poorly characterized proteins, one aim of this thesis was to investigate their import and export mechanisms in detail. In the following, four of these proteins will be described, namely DEAD-box polypeptide 43 (DDX43), DEAD-box polypeptide 59 (DDX59), SRY box 6 (Sox 6) and deleted in breast cancer 1 (DBC-1).

The major aspect of this work then concentrates on the function of Nup358 to analyze which domain of the huge protein mediates nuclear import of these specific proteins or if one domain is responsible for different pathways. The kind of interaction between Nup358 and substrate will be characterized as well.

\subsubsection{Nuclear accumulation of DDX43 and DDX59 requires Nup358}

Two of the Nup358-dependent proteins, DEAD-box polypeptide 43 (DDX43, also named HAGE) and DEAD-box polypeptide 59 (DDX59), contain a DEAD-box-like motif with the amino acid sequence Asp-Glu-Ala-Asp as considered for RNA helicases (Linder et al., 1989; Koonin, 1991). This protein family plays important roles in RNA metabolism like RNA turnover, pre-mRNA splicing and mRNA export (Tseng et al., 1998; Jankowsky et al., 2001; Rocak et al., 2004; Coller et al., 2005). In addition, they unwind RNA structures or dissolve RNA/protein complexes by ATP-hydrolysis (Tanner, 2003).

The sequence of DDX43 displays $55 \%$ homology to DDX 5 (p68), another member of this family and might function in RNA metabolism, cell cycle control and embryogenesis (Martelange et al., 2000). Little is known about the subcellular localization and function, although DDX43 was detected at different levels in a variety of tumor tissues (Mathieu et al., 2010).

To reproduce and confirm the results by Dr. S. Hutten (Hutten, 2007), DDX43 and DDX59 were transiently transfected in HeLa P4 cells. Hence, DDX43-YFP localized to the nucleus in control cells (Figure 3-2 A). After depletion of Nup358, the protein accumulated in the cytoplasm and the numbers of cells displaying a nuclear localization of the reporter protein decreased significantly from $60 \%$ to $13 \%$ (Figure 3-2 B). Similar results were obtained using either an N-terminal CFP-fusion or a much smaller HA-fusion protein (data not shown). 
A

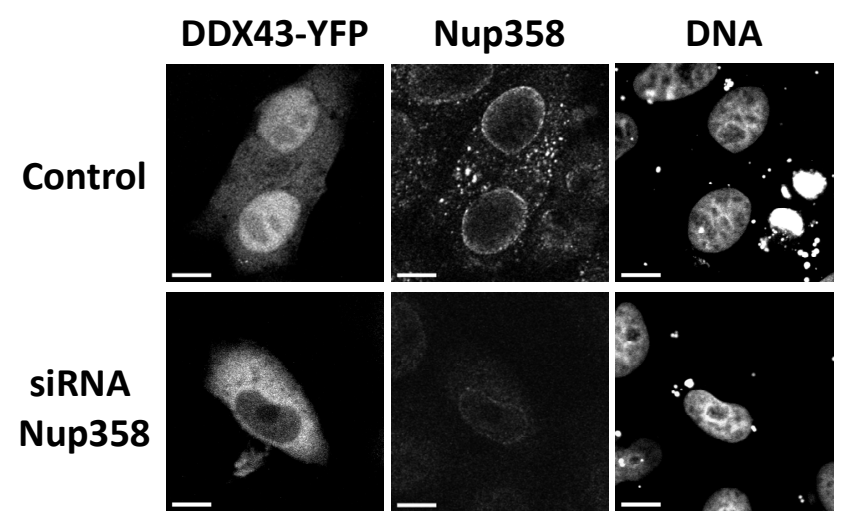

C

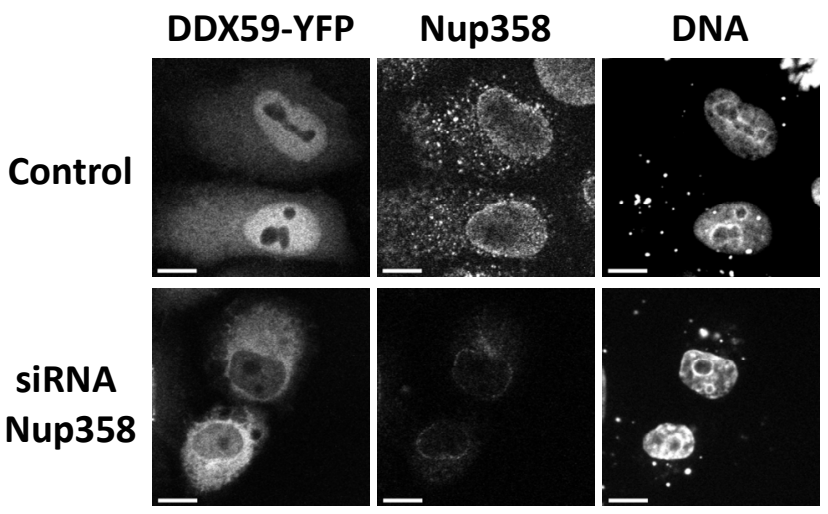

B

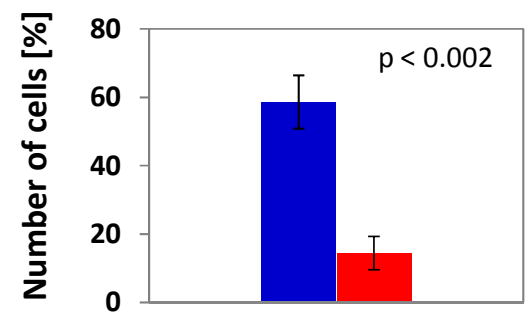

$\mathrm{N}>\mathrm{C}$

- Control siRNA Nup358

D

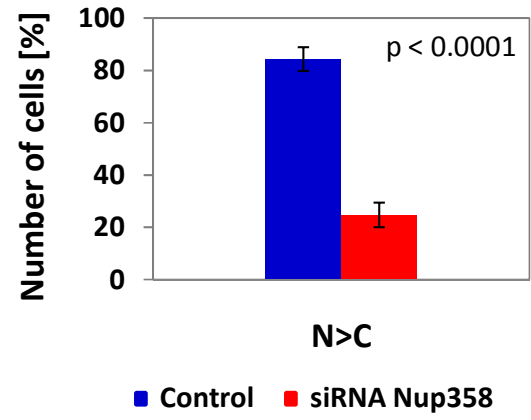

Figure 3-2: Nup358 is required for nuclear accumulation of DDX43 and DDX59.

HeLa P4 cells were transfected with a specific siRNA against Nup358 or kept untreated. Cells were further transfected with plasmids coding for DDX43-YFP (A, B) or DDX59-YFP (C, D). Endogenous Nup358 was detected with an $\alpha$-Nup358 antibody, and DNA was visualized by Hoechst. Distribution of the proteins was analyzed by fluorescence microscopy. For quantitative analysis of DDX43-YFP (B) and DDX59-YFP (D), the distribution of the proteins within the cells were grouped into three categories $N>C, C=N$ and $C>N(N>C$ is shown here). In general, at least 100 equally expressing cells which showed a clear reduction in the level of Nup358 were analyzed. Bars indicate the standard deviation from the mean of three independent experiments. The statistical significance of the data was determined using the student's t-test. Scale bars: $10 \mu \mathrm{m}$. 
In comparison, DDX59-YFP localized to $84 \%$ in the nucleus in control cells whereas after depletion of Nup358 only $24 \%$ showed nuclear localization (Figure 3-2 C+D). C-terminal fusions to CFP- or HA-tags depicted a similar strong reduction of the nuclear localization upon Nup358 depletion which confirmed the specificity of the observed phenotype (data not shown). No effect for control proteins could be observed (Figure 3-8 B).

\subsubsection{DDX43 and DDX59 bind to distinct import factors}

As very little was known about the mechanism of nuclear import of DDX43 and DDX59, we performed binding assays to look for interacting transport factors. DDX43 was expressed as a GST-fusion protein, immobilized to glutathione sepharose and binding of His-tagged importins was analyzed in the absence or presence of RanQ69L-GTP (RanQ69L). The RanQ69L mutant cannot hydrolyze its bound GTP, and releases specifically interacting transport receptors from the immobilized cargo protein. DDX43 could bind to several importins in a Ran-dependent manner. Importin 9 and importin 7 interacted with the protein in the absence, but not in the presence of the Ran-mutant, demonstrating the specificity of the interaction (Figure 3-3 A). Western blot analysis showed a Ran-dependent binding of importin 13. Importin 5, transportin, importin $\beta$ in presence or absence of the adaptor protein importin $\alpha$ did not interact with DDX43.

We also expressed DDX59 as GST-fusion protein, and analyzed binding of recombinant import receptors. Transportin as well as importin 5 and 7 interacted weakly in a Ran-specific manner (Figure 3-3 B). Importin 9, importin 13 as well as importin $\alpha$ did not bind to DDX59. Western blot analysis showed that importin $\beta$ did not associate either (data not shown).

In summary, several import receptors could bind to the GST-fusions of DDX43 and DDX59 in vitro. Nevertheless, a specific interaction with an import receptor in vitro does not necessarily qualify this importin as a functional import mediator in vivo. 
A
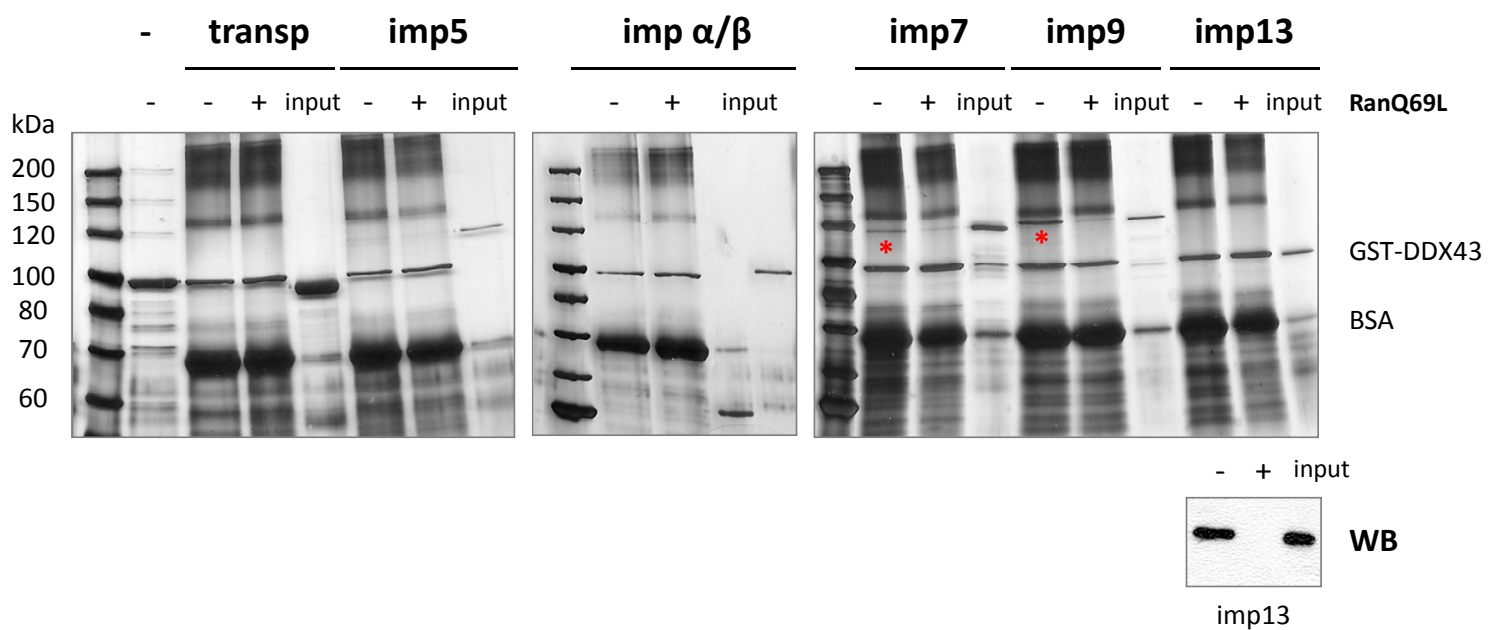

B

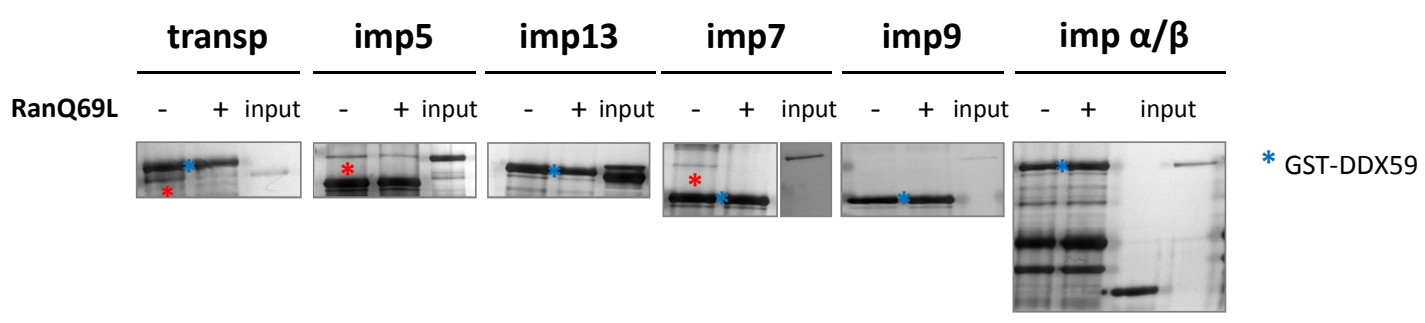

Figure 3-3: DDX43 and DDX59 bind both to distinct import receptors.

GST-DDX43 (A) or GST-DDX59 (B) were immobilized on glutathione sepharose and incubated with importins in the absence (-) or presence (+) of RanQ69L, which had been loaded with GTP. BSA was used as a blocking reagent. Interacting proteins were analyzed by SDS-PAGE, followed by silver staining. The red asterisks mark bound importins. Input corresponds to $10 \%$ of the import receptor used for the binding reaction. A: Western blotting (WB) using an $\alpha$-His-antibody was applied to confirm binding of importin 13 . B: Blue asterisks indicate bound GST-DDX59.

\subsubsection{Depletion of Nup358 causes extranuclear aggregations of Sox 6}

SRY is a founding member of transcription factors called Sox (SRY-related HMG-box) that are implicated in the determination and differentiation in embryonic development, especially in male-specific gonad formation (reviewed in Kamachi et al., 2000; Wilson et al., 2002; Sim et al., 2008). A conserved high-mobility group (HMG-) domain characterizes the protein family mediating DNA binding and protein-protein interactions (Jantzen et al., 1990). The majority of point mutations in XY male-to-female disorders of 
sex development ( $X Y$ DSD) patients are localized in this domain, highlighting its importance (reviewed in Sim et al., 2008). Inhibition of nuclear translocation of SRY and probably other Sox proteins leads to XY DSD (Vilain et al., 2007).

The HMG-domain is target of different post-translational modifications like sumoylation of SRY-box 6 (Sox 6) (Fernandez-Lloris et al., 2006) which represses transcription activity. Moreover, Sox 6 is involved in maintaining the physiology of muscle tissue (Hagiwara et al., 2000) or regulating gene expression for chondrogenesis (Lefebvre et al., 1998).

To reproduce and confirm the Nup358-dependent accumulation of Sox 6 (Hutten, 2007), Sox 6-YFP was transfected in control and Nup358-depleted cells, and its localization was analyzed. The protein located to the nucleus in control cells mostly in a speckle-like distribution (better seen in Figure 3-6 B). After depletion of Nup358, a subset of Sox 6 still localized in the nucleus. Additionally, large extranuclear aggregates could be observed (Figure 3-4, red arrows). As this phenotype made it difficult to group cells according to the three categories $\mathrm{N}>\mathrm{C}, \mathrm{N}=\mathrm{C}$ and $\mathrm{C}>\mathrm{N}$, no quantification was performed here.

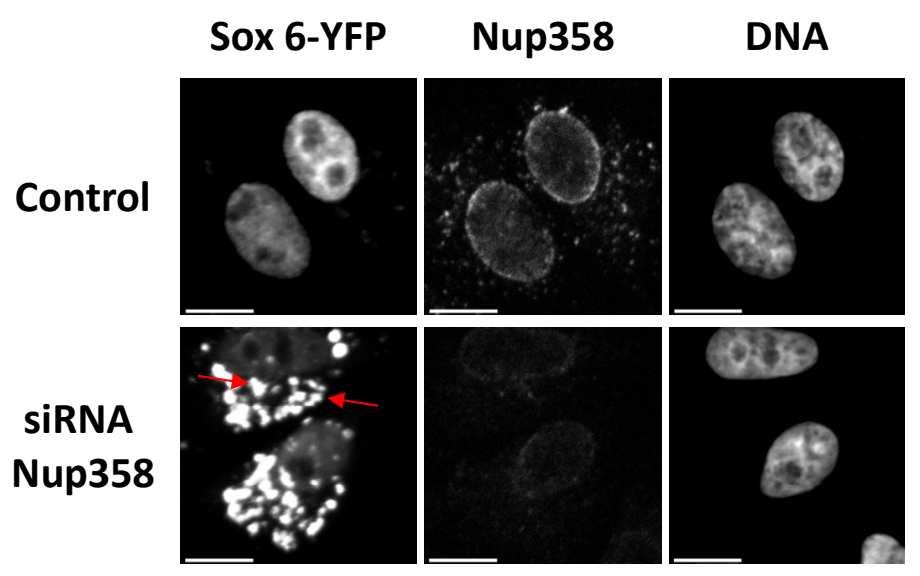

Figure 3-4: Depletion of Nup358 causes extranuclear aggregations of Sox 6.

Control or Nup358-depleted cells were transfected with cDNA coding for Sox 6-YFP. Cells were fixed, Nup358 was detected with a specific antibody, and DNA was visualized by Hoechst. Localization was analyzed by fluorescence microscopy. Red arrows mark the aggregates. Scale bars: $10 \mu \mathrm{m}$. 


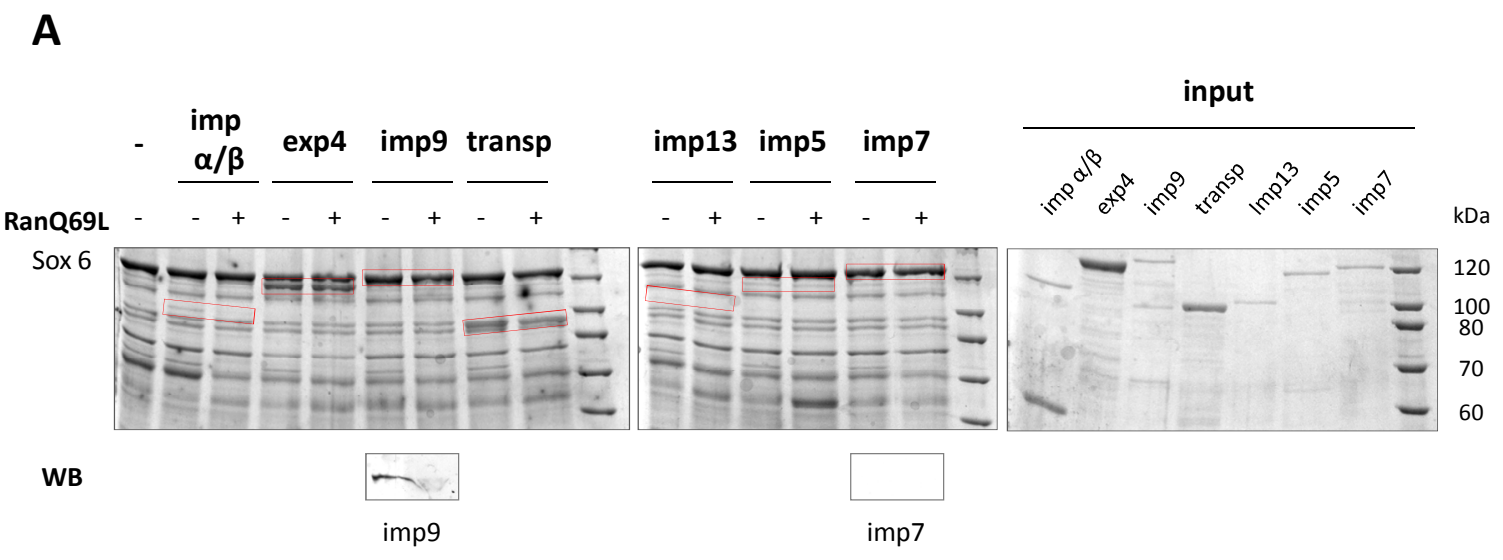

B

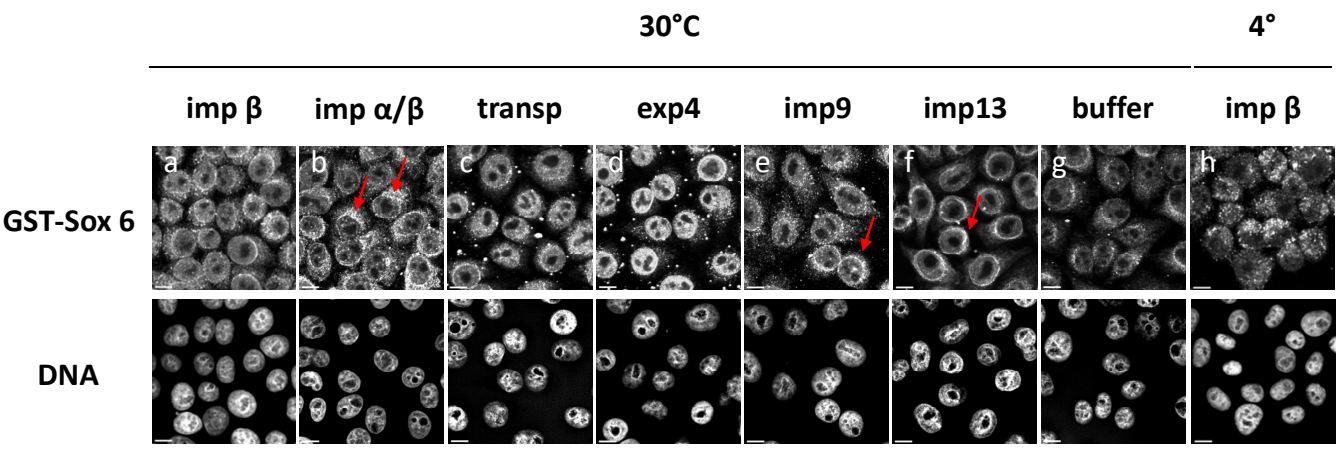

Figure 3-5: Various import receptors bind to Sox 6 and mediate nuclear import in vitro.

A: GST-Sox 6 was immobilized on glutathione sepharose, and incubated with importins in the absence (-) or presence (+) of RanQ69L loaded with GTP. BSA was used as a blocking reagent. Interacting proteins were analyzed by SDS-PAGE, followed by silver staining. The red boxes mark putative binding of the importins. Western blotting (WB) using an $\alpha$-His-antibody was performed to confirm binding of importin 7 and importin 9. The input corresponds to $2 \%$ of the import receptor used for the binding reaction. B: Digitonin-permeabilized HeLa P4 cells were incubated with GST-Sox 6 as an import substrate and with equimolar concentrations of import receptors in the presence of $2 \mu \mathrm{m}$ Ran wt at $30^{\circ} \mathrm{C}$ or $4^{\circ} \mathrm{C}$. After detection of GST-Sox 6 with an $\alpha$-GST-antibody and staining of the DNA, import was analyzed by fluorescence microscopy.

\subsubsection{Different import factors can mediate nuclear import of Sox 6}

Nuclear import of other proteins from the Sox family was analyzed intensively, but nothing was known about nuclear import of Sox 6 so far. In order to search for interacting NTRs, binding assays with purified recombinant proteins were carried out. Thus, GST-Sox 6 was immobilized to glutathione sepharose, and incubated with import factors in the absence or presence of RanQ69L. Importin $\alpha / \beta$ binding was rather weak to GST-Sox 6 compared to transportin which displayed Ran-independent interaction 
(Figure 3-5 A). Western blot confirmed binding of importin 9. Importin 7 did not associate to GST-Sox 6 which was also revealed in western blot analysis (Figure 3-5 A, WB). Importin 13 and importin 5 did not interact either.

As recently demonstrated, the export factor exportin 4 mediates nuclear import of SRY and Sox 2 (Gontan et al., 2009). In this context, binding of recombinant exportin 4 to Sox 6-YFP was also tested. Indeed, the proteins interacted with each other, but RanQ69L did not abolish the interaction (Figure 3-5 A).

As the SRY protein is transported actively into the nucleus by binding directly to importin $\beta$ (Sudbeck et al., 1997), we also tested interaction of importin $\beta$ to Sox 6 without importin $\alpha$ as an adaptor protein. Indeed, Sox 6 interacted strongly with importin $\beta$ in a Ran-dependent manner whereas presence of importin $\alpha$ rather impeded the interaction with importin $\beta$ (Appendix, Figure I).

To answer the question which import receptor indeed functioned as import mediator for Sox 6 in the cellular system, we performed in vitro import assays using permeabilized HeLa P4 cells and recombinant import factors, GST-Sox 6, Ran and an ATP-regenerating system (Adam et al., 1990). Importin $\beta$ alone could mediate nuclear uptake of Sox 6 as the GST-signal was stronger than with importin $\alpha / \beta$ (Figure 3-5 $B$, image $a+b$ ). Interestingly, the protein seemed to assemble at the outer nuclear membrane or at the entrance of the nuclear pore (red arrow). The uptake with transportin was very efficient with a strong accumulation in the nucleus (image c). The import reaction with exportin 4 resulted in an even brighter nuclear signal (image d). Importin 9 showed less efficient import (image e) which was in accordance with the weaker binding in the binding assays. Again, an accumulation at the nuclear rim was observed. Importin 13 could support weak import of GST-Sox 6 which was in contrast to the result of the binding assays (image f). Slight nuclear uptake was observed in the absence of exogenous transport receptors (image $g$, buffer) which could be explained by leftovers of endogenous import receptors in the cytoplasm.

To exclude passive diffusion of GST-Sox 6 into the nucleus, each reaction was also carried out at $4^{\circ}$ as active import does not take place at this temperature. Uptake of GST-Sox 6 with importin $\beta$ at $4^{\circ}$ (image h) was clearly reduced compared to $30^{\circ} \mathrm{C}$ 
(image a). In summary, transportin and exportin 4 and to a lesser extent importin $\beta$ seem to represent the main import factors of Sox 6 at least in vitro.

\subsubsection{The C-terminal half of Sox 6 contains several NLSs}

As mentioned earlier, import of the Sox 6 related proteins SRY and Sox 2 can be mediated by exportin 4, and this interaction can be abolished by a mutation from arginine to glycine in the HMG-box (Gontan et al., 2009). As the sequence homology to SRY in this region was relatively high, the arginine within the HMG-box was mutated to glycine to achieve Sox 6 R600G-YFP. Hence, binding of recombinant exportin 4 was tested to Sox 6 wild type and mutant after immunoprecipitations using the GFP-nanotrap. Exportin 4 could bind to wild type but not to the mutant anymore (Figure 3-6 A). Note that in contrast to the in vitro binding assay (Figure 3-5 A), binding of exportin 4 was reduced by addition of RanQ69L. No association of exportin 4 to the mock GFP-nanotrap (beads) was observed. Additionally, Sox 6-R600G-YFP was transfected into HeLa P4 cells (Figure 3-6 B), resulting in nuclear localization. This suggests that more than one import receptor can mediate import of Sox 6 in vivo.

A

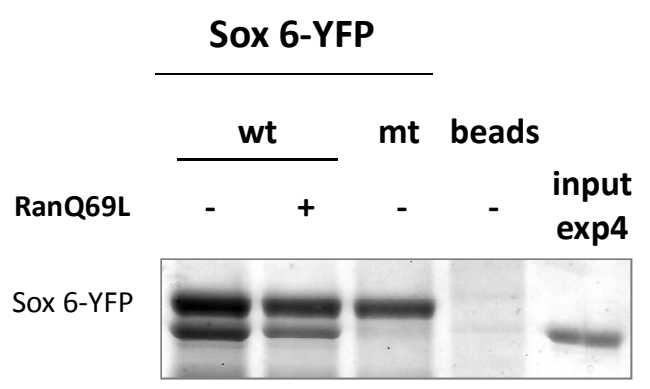

B

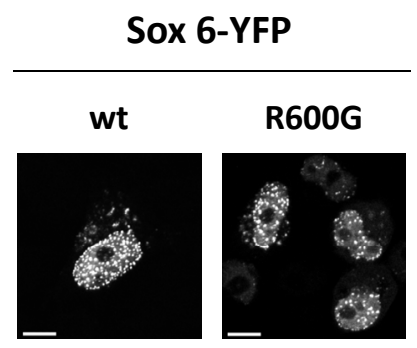

Figure 3-6: Sox 6 R600G cannot bind exportin 4 anymore, but can still be imported.

A: Sox 6-YFP (wt) and Sox 6-R600G-YFP (mt) were immunoprecipitated from 293T HEK cells using the GFP-nanotrap and incubated with exportin 4 in the absence (wt, mt) (-) or presence (wt) (+) of RanQ69L loaded with GTP. Interaction of exportin 4 was analyzed by SDS-PAGE, followed by colloidal coomassie staining. The input corresponds to $10 \%$ of exportin 4 used for the binding reaction. B: HeLa P4 cells were transfected with plasmids coding for Sox 6-YFP and Sox 6-R600G-YFP and localization was analyzed by fluorescence microscopy. Scale bars: $10 \mu \mathrm{m}$. 
To narrow down the binding region for importin $\beta$ and transportin, we divided the protein into an $\mathrm{N}$-terminal and several C-terminal fragments (Appendix, Figure II A), and analyzed their subcellular localization as GFP-fusion proteins after transient transfection. The $\mathrm{N}$-terminal fragment located to the cytoplasm (Appendix, Figure II B, image b) whereas all C-terminal fragments localized to the nucleus (images c-i), suggesting several active NLSs within this part of Sox 6 . Several mutations in putative NLS-sequences did not result in cytoplasmic accumulation (Appendix, Figure II A+B).

Together, this points to distinct nuclear import pathways involved in mediating nuclear uptake of Sox 6 .

\subsubsection{Nuclear localization of DBC-1 depends on Nup358}

The most prominent candidate in our screen for Nup358-dependent import is the nuclear $130 \mathrm{kDa}$ protein 'deleted in breast cancer 1' (DBC-1), also KIAA 1967 or p30 (Hutten, 2007) (Figure 3-7 A: schematic description of the protein; Appendix, Figure III: amino acid sequence).

The gene encoding DBC-1 was originally identified on human chromosome $8 \mathrm{p} 21$, a region that is often homozygously deleted in breast and other cancers like lung (Hamaguchi et al., 2002). The protein was also shown to be upregulated in breast carcinomas (Richardson et al., 2006), and no altered expression was found in prostate cancer (Fu et al., 2009).

The cellular functions of DBC-1 are just emerging, like the direct interaction to Sirt1, a member of the sirtuin family. Here, DBC-1 inhibits the deacetylase activity resulting in acetylation of p53 (Kim et al., 2008; Zhao et al., 2008). This activation of p53 leads to apoptosis of the cell. Further, caspase activity generates two C-terminal fragments of DBC-1 (p120 and p60) which relocalizes to the cytoplasm and mitochondria to facilitate cell death in a not yet known mechanism (Sundararajan et al., 2005). In contrast to these proapoptotic functions leading to tumor suppression, DBC-1 binds ligand-independently to the estrogen receptor $\alpha$ (ER $\alpha)$, the predominant receptor isoform in breast cancer. 
A

DBC-1

aa 1

B

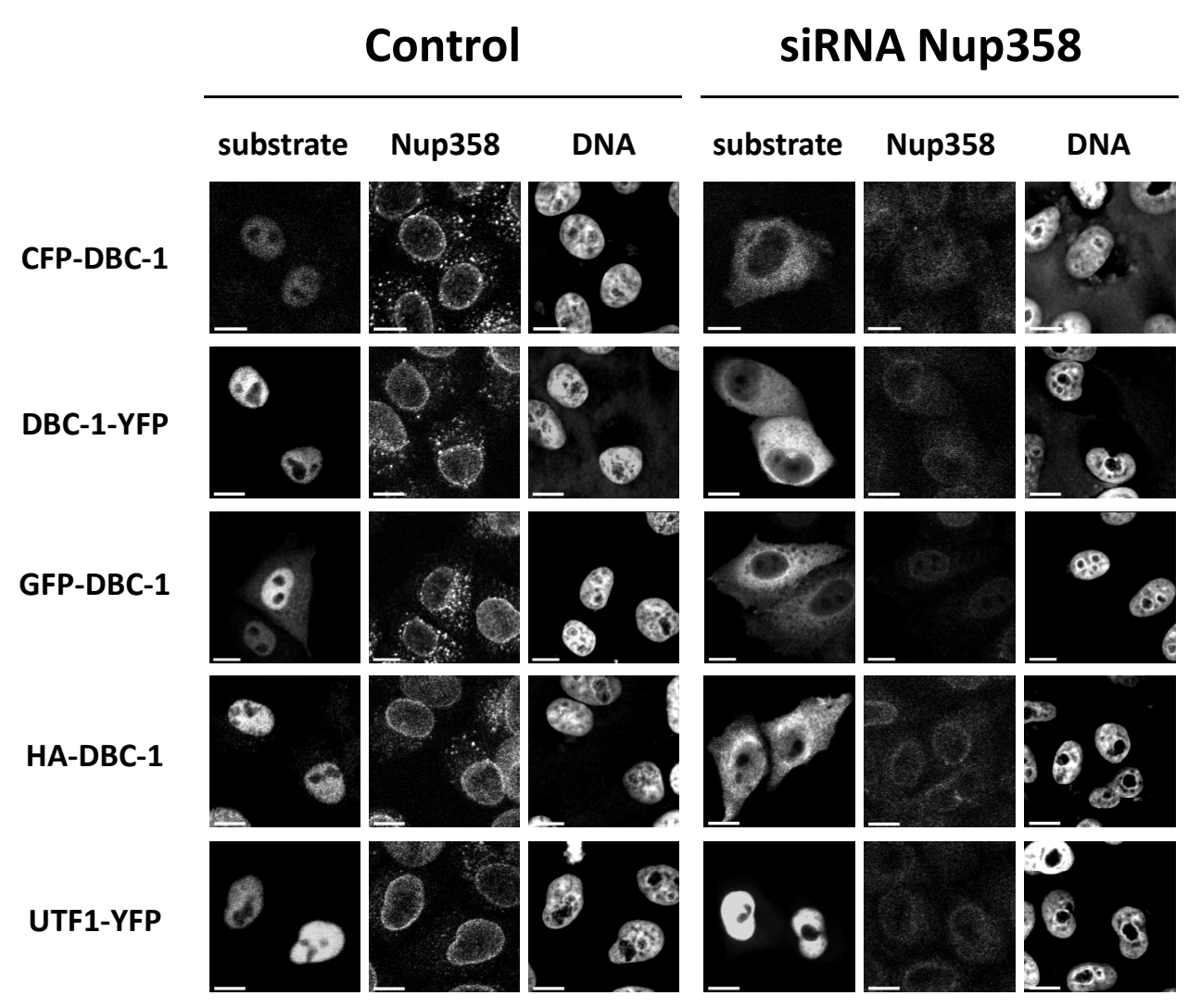

C

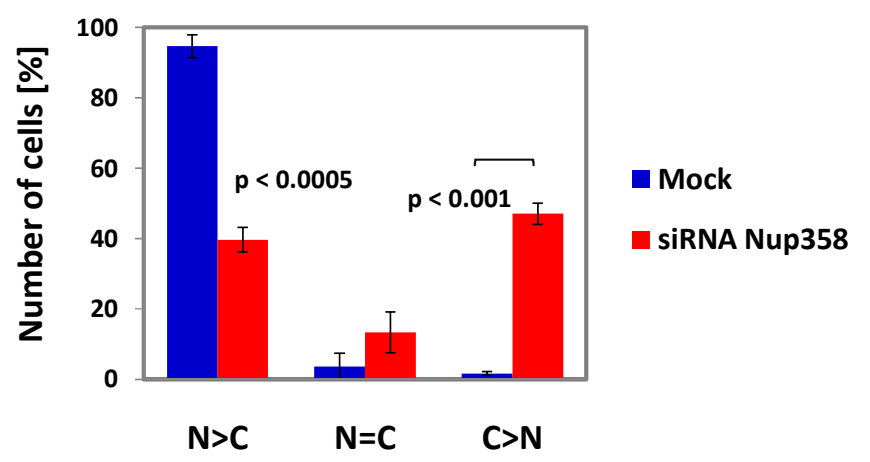


This interaction inhibits hormone-independent apoptosis promoting breast cancer cell growth and survival (Trauernicht et al., 2007). In addition, DBC-1 binds to the androgen receptor (AR), coactivating its transcriptional activity in prostate cancer (Fu et al., 2009) and to the methyltransferase SUV39H1 (Li et al., 2009). These functions suggest a role in the regulation of cell growth, but the physiological function of DBC-1 still needs to be analyzed.

Nuclear import of DBC-1 depends on Nup358 (Hutten, 2007). To test whether the nature or the position of the tag affected Nup358 dependence, different variants of DBC-1 were transiently transfected in control and Nup358-depleted cells. Hence, DBC-1 localized into the nucleus in control cells, but was found in the cytoplasm of cells lacking Nup358 (Figure 3-7 B). This phenotype was not dependent on either the nature of the tag (FP or $\mathrm{HA}$ ) or the location of the tag (N- or C-terminal). A quantification of cells transfected with CFP-DBC-1 demonstrated a significantly strong decrease of cells displaying nuclear localization of the reporter protein from $90 \%$ to $40 \%$ and a corresponding increase of cells with a cytoplasmic accumulation of CFP-DBC- 1 from $2 \%$ in control cells to $47 \%$ in knockdown cells (Figure 3-7 C). Similar results could be obtained with GFP-DBC-1 (for example Figure 3-11 A, 3-12 B).

Importantly, the majority of proteins derived from the LIFEdb did not depend on Nup358 in their nuclear import, as shown for UTF1-YFP (upstream transcription factor 1) (Figure 3-7 B).

Figure 3-7: Nuclear localization of DBC-1 depends on Nup358.

A schematic description of DBC-1 is shown in A. Domains were predicted by PROSITE and BLAST protein homology searches (Sundararajan et al., 2005). B: Control or Nup358-depleted HeLa P4 cells were transiently transfected with plasmids coding for CFP-DBC-1, DBC-1-YFP, HA-DBC-1, GFP-DBC-1 or UTF1-YFP. After immunostaining using an $\alpha$-Nup358 antibody and an $\alpha$-HA-antibody for HA-DBC-1, localization of the proteins was analyzed by fluorescence microscopy. Scale bars: $10 \mu \mathrm{m}$. C: The quantification shows the mean distribution of CFP-DBC- 1 in the three categories $\mathrm{N}>\mathrm{C}, \mathrm{N}=\mathrm{C}$ and $\mathrm{C}>\mathrm{N}$. Error bars indicate the standard deviation from the mean of three independent experiments. > 100 cells were analyzed for each single experiment. The statistical significance of the data was determined using the student's t-test. 


\subsubsection{Nuclear import of Sirt1 does not require Nup358}

As DBC-1 interacts with Sirt1 (Zhao et al., 2008) and ER $\alpha$ (Trauernicht et al., 2007), it was interesting to analyze their nuclear import regarding Nup358 dependence. Sirt1 localizes exclusively to the nucleus in male germ cells and COS-7 cells after transient transfection (McBurney et al., 2003; Sakamoto et al., 2004) although there are reports about the cytoplasmic accumulation in murine pancreatic $\beta$ cells (Moynihan et al., 2005). Tanno and Co-workers identified two functional NLS in the sequence of murine Sirt1 and nuclear localization required both signals (Tanno et al., 2007).

After transient transfection in control as well as in Nup358-depleted cells, mCherry-Sirt1 localized to the nucleus (Figure 3-8). Proteins may also be imported passively by a mechanism described as piggyback import (Kang et al., 1994). So we tested in cotransfection experiments in Nup358-depleted cells if one of the proteins got transported to the nucleus through an interaction with the other.

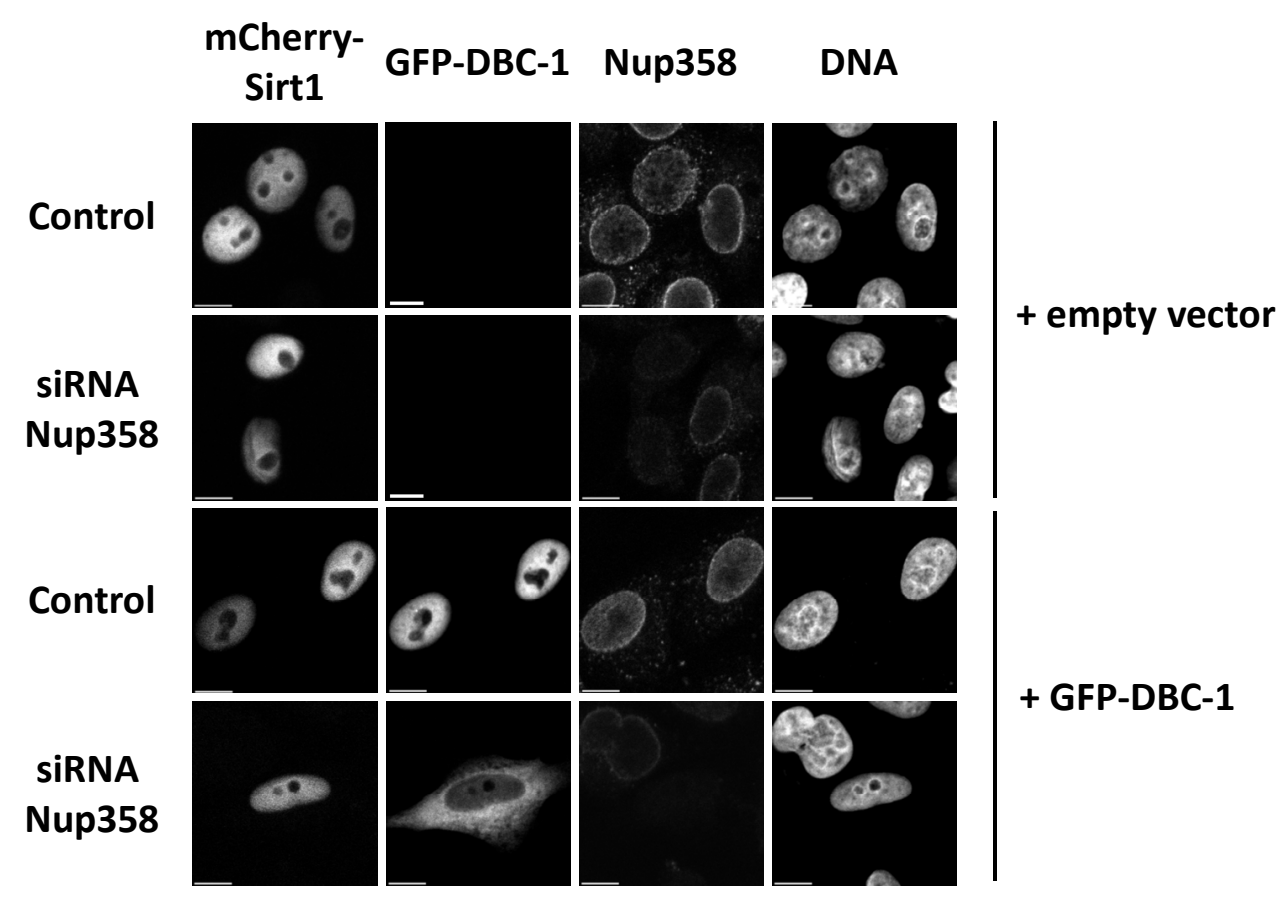

Figure 3-8: Nuclear import of Sirt1 requires neither neither Nup358 nor DBC-1.

Control or Nup358 siRNA-treated cells were cotransfected with cDNA coding for mCherry-Sirt1 and an empty vector or GFP-DBC-1, as indicated. After immunostaining of Nup358 and detection of the DNA, localization of the tagged proteins was visualized by fluorescence microscopy. Scale bars: $10 \mu \mathrm{m}$ 
The Nup358-independent import of Sirt1 could not compensate for the impaired import of DBC-1 as the protein was still cytoplasmic. Likewise, Sirt1 localized in the nucleus although DBC-1 accumulated into the cytoplasm. To exclude sterical obstruction because of the FP-tags, Sirt1 with the smaller myc-tag was cotransfected with GFP-DBC-1, but did not influence the behavior of the proteins in Nup358-depleted cells (data not shown). In fact, both proteins were transported to the nucleus independently of each other. Similarly, the estrogen receptor $\alpha$ (ER $\alpha)$ did not show Nup358-dependent nuclear import and no piggyback mechanism was observed for ER $\alpha$ and DBC-1 (data not shown).

This and the fact that only a small set of proteins derived from the LIFEdb were identified as Nup358-dependent underlines the specific role of Nup358 in cargo-specific transport pathways.

\subsubsection{The importin $\alpha / \beta$ complex mediates nuclear import of DBC-1}

A major emphasis of this thesis lies on the analysis of DBC-1. To our knowledge, the nuclear import mechanism of this protein has not yet been analyzed so far. As already shown, passive import by interaction with Sirt1 and ER $\alpha$ could be excluded. Further, PROSITE and BLAST protein homology searches had yielded a domain map of DBC-1 (Sundararajan et al., 2005) containing next to other domains a putative NLS within amino acids 200-219 (Figure 3-9 A).

In order to confirm and characterize this putative NLS within amino acids 200-219, several fragments of DBC-1 with or without the putative NLS were analyzed for their subcellular localization after transfection into HeLa P4 cells. A scheme and information about their localization is shown in Figure 3-9 A. The N-terminal fragments reaching from amino acid 1-199 (-NLS) or 1-220 (+NLS) were fused to double GFP to avoid passive diffusion. The C-terminal fragments from amino acid 200-923 (+NLS) and 220-923 (-NLS) contained a single GFP. Although fragment aa 1-199 did not contain any predicted NLS, it localized to the nucleus (Figure 3-9 B, image a), and this localization was not different by fusion to a larger tag (data not shown). 
A

Localization

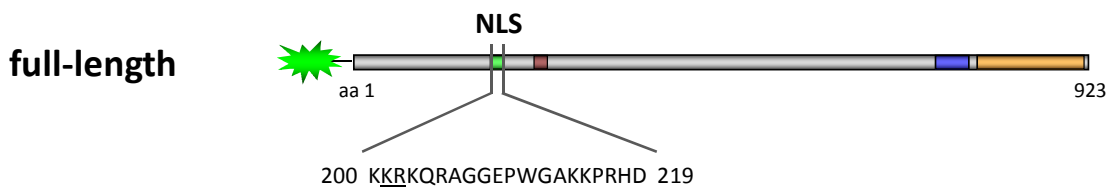

$\mathbf{N}$

200 KKRKQRAGGEPWGAKKPRHD 219

aa 1-199

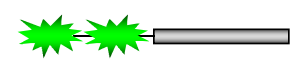

aa 1-220

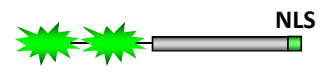

aa $200-923$

NLS

aa $220-923$

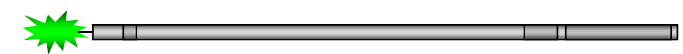

$\mathbf{N}$

N

$\mathbf{N}$

C

B

GFP-DBC-1 fragments

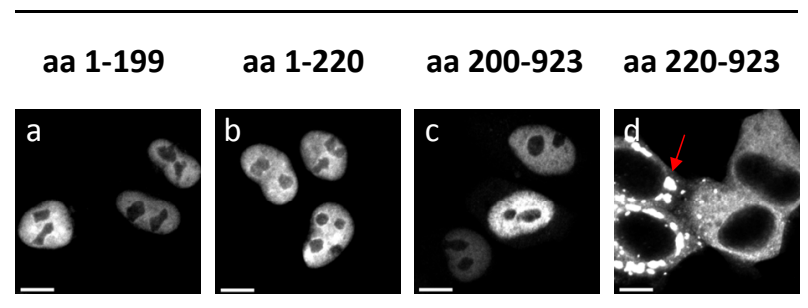

C

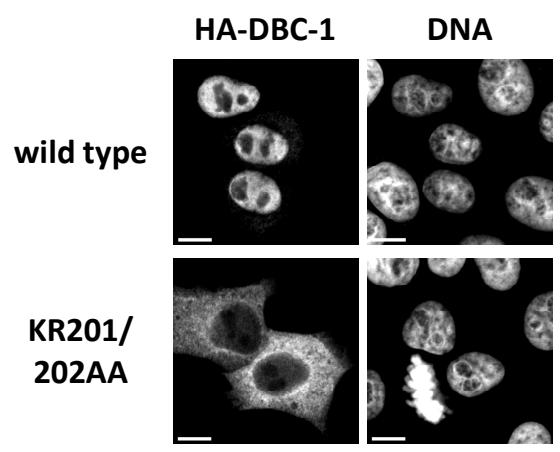

Figure 3-9: DBC-1 has a functional NLS.

Schematic description of DBC-1 full-length and fragments containing GFP-tags and the respective subcellular localization is shown in A. B: HeLa P4 cells were transiently transfected with plasmids coding for GFP $_{2}$-DBC-1 aa 1-199, GFP 2 -DBC-1 aa 1-220, GFP-DBC-1 aa 200-923 and GFP-DBC-1 aa $220-923$ and analyzed. The red arrow indicates aggregates in the cytoplasm of GFP-DBC-1 aa 220-923 in image d. C: HeLa P4 cells were transiently transfected with HA-DBC-1 wild type and KR201/202AA, stained using an $\alpha-H A$-antibody and Hoechst for DNA. Localization of the proteins was determined by fluorescence microscopy. Scale bars: $10 \mu \mathrm{m}$. 
As expected, the fragments containing the predicted NLS accumulated in the nucleus (image $b+c$ ) whereas the C-terminal fragment from aa 220-923 without the NLS stayed cytoplasmic (image d) displaying aggregates in some cells (red arrow).

The region around amino acid 200 contains several basic residues namely KKRKQR (Figure 3-9 A). Transfection of a NLS-mutant protein (DBC-1 KR201/202AA) either GFP- or HA-tagged into HeLa P4 cells resulted in a strong cytoplasmic localization (Figure 3-9 C). According to these data, the putative NLS which is sufficient and necessary to mediate nuclear import of full-length DBC-1 lies within amino acids 200-219.

Next, interaction of distinct import receptors, namely endogenous importin $\alpha$, importin $\beta$ and transportin, were analyzed either with full-length, the NLS-mutant or the truncations aa 1-199 (-NLS), aa 1-220 (+NLS) and aa 200-923 (+NLS) of DBC-1. For this, immunoprecipitations of transiently transfected GFP-fusion proteins in 293T HEK cells were carried out in the absence or presence of RanQ69L by using the GFP-nanotrap. Precipitated GFP-proteins are shown in Figure 3-10 A. Full-length DBC-1 interacted with endogenous importin $\alpha$ and importin $\beta$ in contrast to KR201/202AA, which did not bind importin $\alpha$ anymore. Here, binding of importin $\beta$ was reduced. Both wild type and NLS-mutant precipitated transportin even though the full-length protein showed lower interaction due to less bound DBC-1. Remarkably, the short N-terminal fragment with the NLS exhibited a very strong binding of all importins which was observed in several independent experiments. The importins interacted only weakly with the short N-terminal fragment, aa 1-199, missing the NLS. Surprisingly, the long C-terminal fragment containing the NLS, aa 200-923, could precipitate importin $\alpha$ very efficiently, but less importin $\beta$. GFP alone as negative control did not interact with any of the importins. Furthermore, RanQ69L abolished the interaction of importin $\beta$ and transportin with all proteins.

In summary, importin $\alpha$ interacted with DBC-1 via amino acids $200-220$ and the mutation abolished its binding. Apparently, the binding of importin $\beta$ alone and transportin did not suffice to mediate import of the mutant pointing to an importin $\alpha / \beta$-dependent nuclear import of DBC-1. 
A total lysate GFP-nanotrap

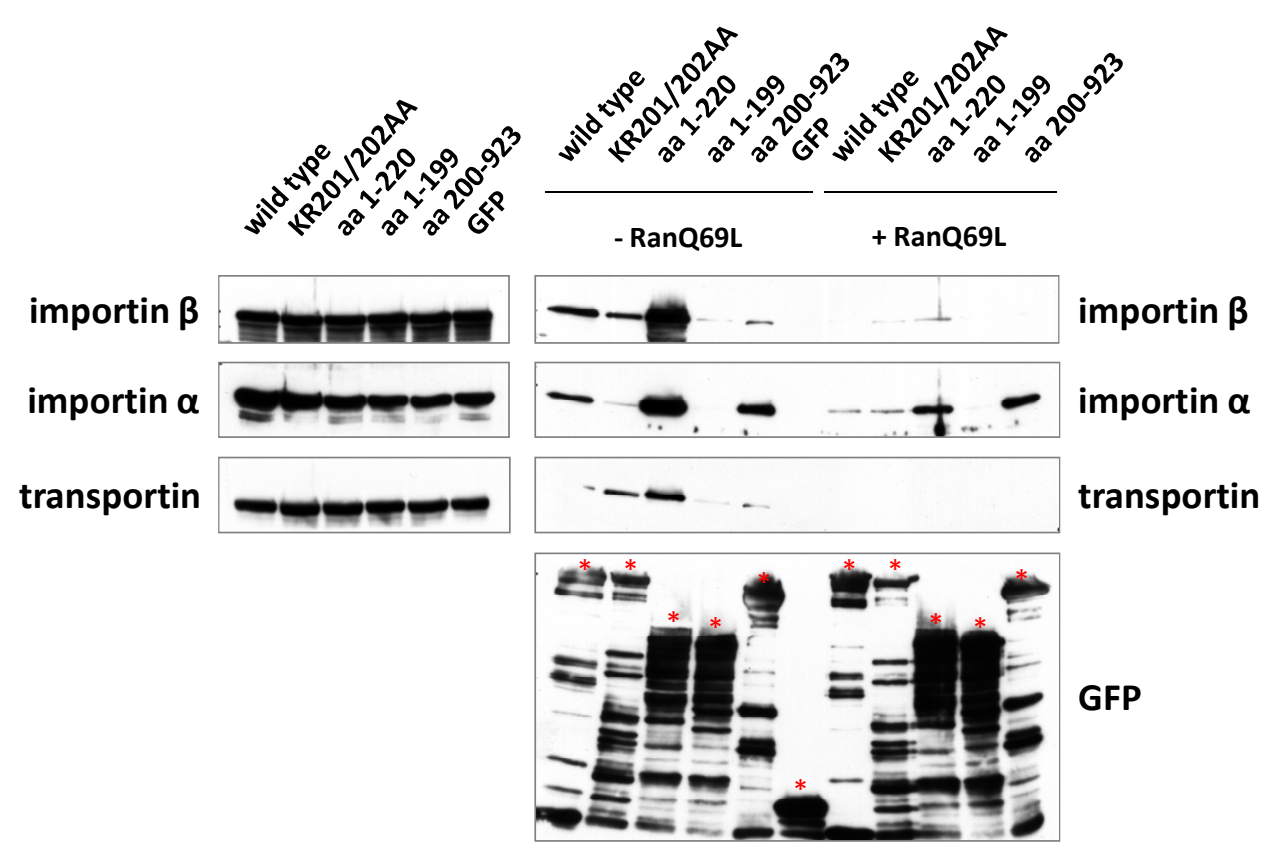

B

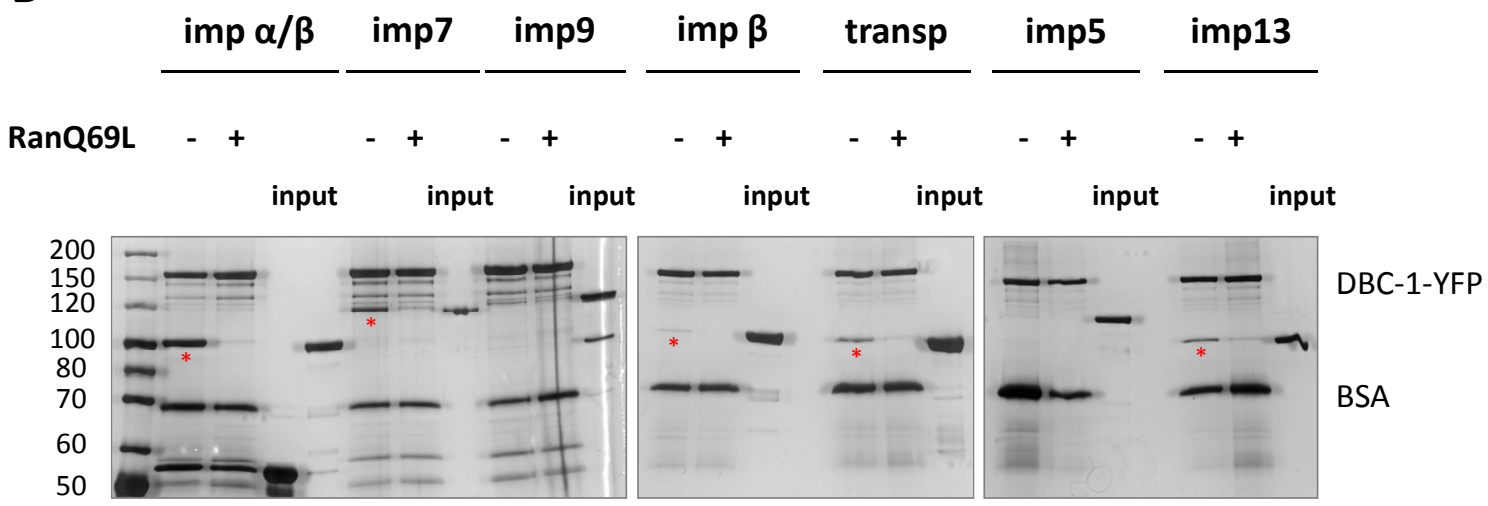

Figure 3-10: Several importins bind to DBC-1.

293T HEK cells were transiently transfected with (A) GFP-DBC-1, GFP-DBC-1 KR201/202AA, GFP $_{2}$-DBC-1 aa 1-220, GFP 2 -DBC-1 aa 1-199, GFP-DBC-1 aa 220-923 and GFP or (B) DBC-1-YFP. GFP-proteins were immunoprecipitated using the GFP-nanotrap. A: Interaction of endogenous importin $\alpha$, importin $\beta$ and transportin was analyzed by SDS-PAGE, followed by western blotting using antibodies against importin $\alpha$, importin $\beta$ and transportin. Bound GFP-proteins (red asterisks) were detected with an $\alpha$-GFP-antibody. The input corresponds to $1 \%$ of total lysate used for the binding reaction. B: DBC-1-YFP bound to the GFP-nanotrap was incubated with different import receptors. Binding of the proteins was analyzed by SDS-PAGE, followed by silver staining. The red asterisk labels interacting importins. The input corresponds to $10 \%$ import receptors used for the binding reaction. BSA was used as a blocking reagent. Both experiments (A, B) were performed in absence (-) or presence $(+)$ of RanQ69L, which had been loaded with GTP. 
In order to identify other interacting transport factors, DBC-1-YFP expressed in 293T HEK cells was immunoprecipitated using the GFP-nanotrap. The protein bound to the sepharose was incubated with different recombinant import receptors in the absence or presence of RanQ69L-GTP.

Importin $\beta$ interacted more efficiently with $\mathrm{DBC}-1$ in the presence of the adaptor molecule importin $\alpha$ than on its own (Figure 3-10 B). Importin 7, importin 9, transportin and importin 13 slightly bound to DBC-1 only, whereas importin 5 did not interact at all. All interactions of DBC-1-YFP with the importins were specific as RanQ69L inhibited binding of the receptors. Further, binding of importin 13 to the NLS-mutant was tested, but the interaction was not weakened or abolished in contrast to association of importin $\alpha$ (data not shown), suggesting that nuclear import of DBC-1 is mediated by the importin $\alpha / \beta$ complex.

\subsubsection{Importins are not the only rate-limiting factors in nuclear import of DBC-1}

Importin $\beta$ has been demonstrated to be rate-limiting for nuclear import of cNLS-substrates in Nup358-depleted cells, suggesting that the nucleoporin promotes utilization of the transport receptors (Hutten et al., 2008). As reported, a shuttling-construct consisting of NES-GFP 2 -CNLS with predominantly nuclear location in control cells accumulated in the cytoplasm upon depletion of Nup358 (Hutten et al., 2008) (Figure 3-11 A). Overexpression of importin $\beta$ could rescue the nuclear localization of NES-GFP 2 -CNLS due to increased concentration of the importin at the cytoplasmic face of the nuclear pore. This is specific for importin $\beta$ as transportin failed to rescue the import defect.

We now investigated whether import of DBC-1 could also be rescued by overexpression of importins in Nup358-depleted cells. Thus, GFP-DBC-1 was cotransfected with HA-importins and the localization of DBC-1 was analyzed in dependence of Nup358 and importin overexpression. Neither HA-importin $\alpha$ nor HA-importin $\beta$ could compensate the import defect (Figure 3-11 $\mathrm{B}+\mathrm{C}$ ), although they interacted strongly and specifically with DBC-1 in the binding assays (Figure 3-10 A+B). 
A

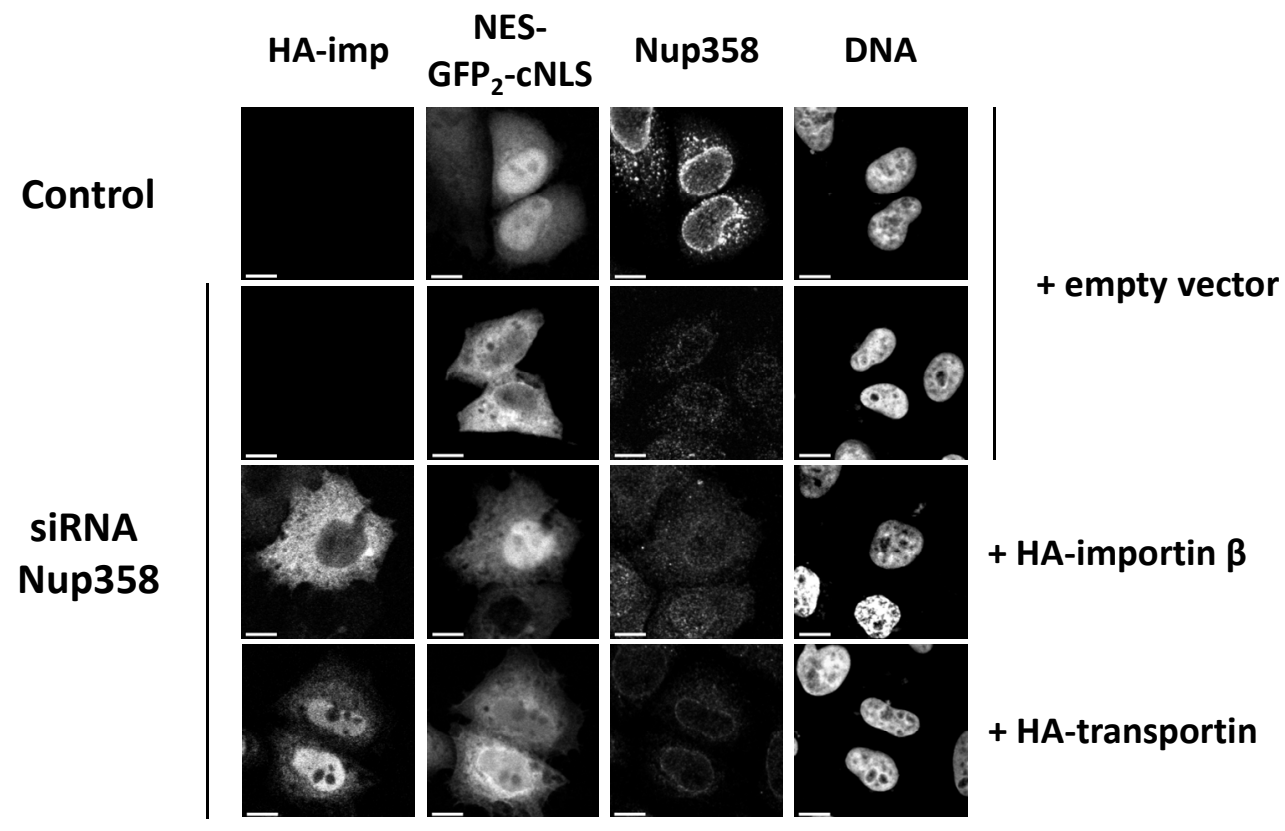

B

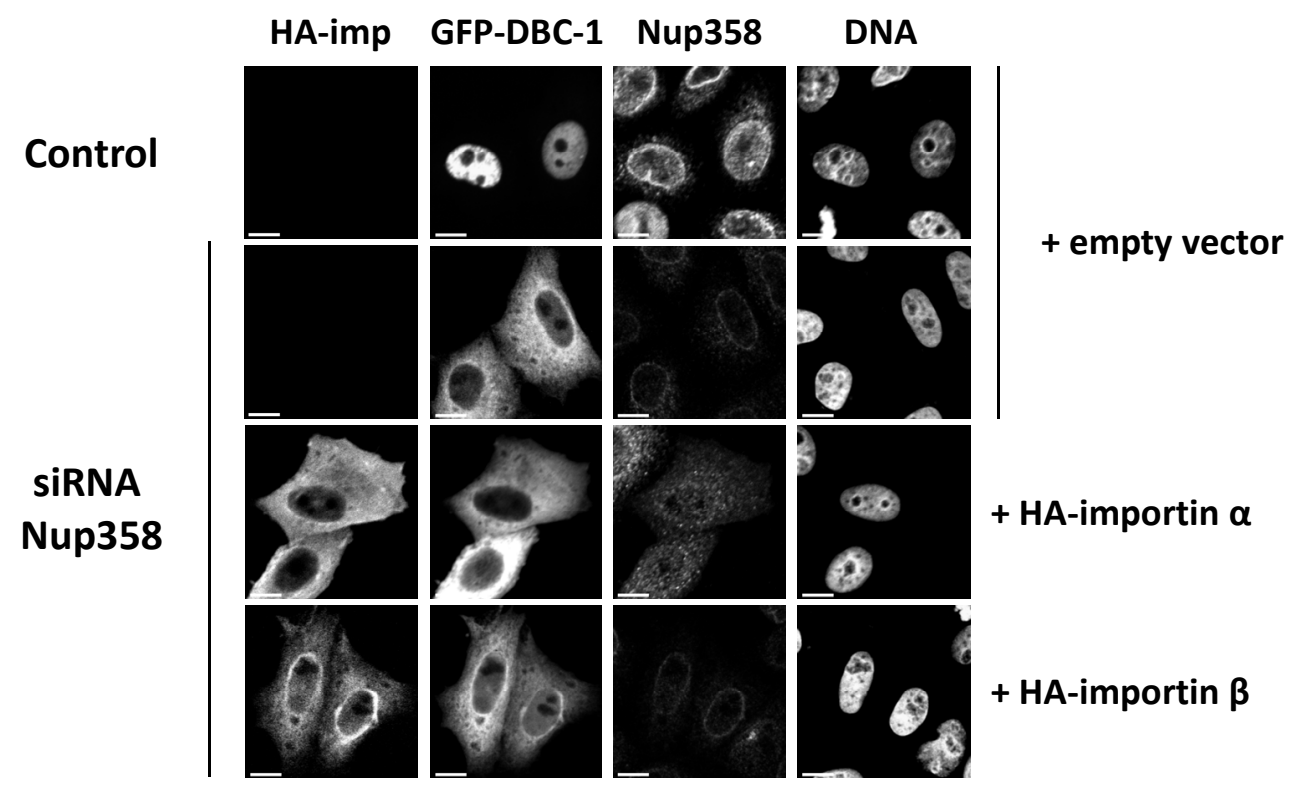

C

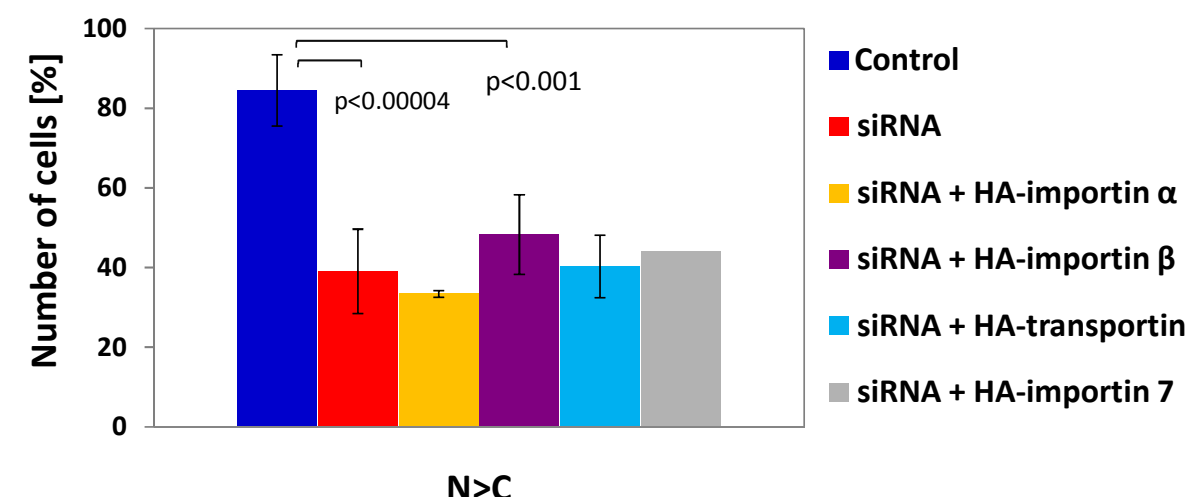


The number of cells with DBC-1 in the nucleus dramatically decreased from $84 \%$ in control cells to $39 \%$ in Nup358-depleted cells (Figure 3-11 C). The presence of importin $\alpha$ did not alter its subcellular localization (34\%). Similarly, HA-importin $\beta$ did not significantly increase nuclear import of DBC-1 (49\%). Likewise, overexpression of HA-transportin (40\%) or HA-importin 7 (44 \%) could not compensate for the knockdown effect.

Neither importin $\beta$ nor any other import factor tested here are the sole rate-limiting factor for nuclear import of DBC-1 in Nup358-depleted cells in contrast to the NLS-reporter protein. This suggests the involvement of additional factors or a direct interaction with Nup358.

Figure 3-11: Importins are not the only rate-limiting factors for nuclear import of DBC-1 in cells depleted of Nup358.

Control or Nup358-depleted HeLa P4 cells were cotransfected with NES-GFP2-CNLS (A) or GFP-DBC-1 (B) with an empty vector or HA-importin $\beta(A, B)$, HA-transportin (A) or HA-importin $\alpha(B)$, as indicated. An $\alpha$-Nup358 and an $\alpha$-HA-antibody were used for immunofluorescence, and DNA was visualized by Hoechst. Localization of the proteins was analyzed by fluorescence microscopy. Scale bars: $10 \mu \mathrm{m}$. C: As in (B), GFP-DBC-1 was cotransfected with HA-importin $\alpha$, HA-importin $\beta$, HA-transportin or HA-importin 7. Distribution of $\mathrm{DBC}-1$ was grouped into $\mathrm{N}>\mathrm{C}, \mathrm{N}=\mathrm{C}$ or $\mathrm{C}>\mathrm{N}$ (only $\mathrm{N}>\mathrm{C}$ is shown here) for $>100$ control or Nup358 siRNA-treated HeLa P4 cells. Error bars depict the standard deviation from the mean of three independent experiments. The statistical significance of the data was determined using the student's t-test. 
A

Control

aa 1-199

aa $1-220$

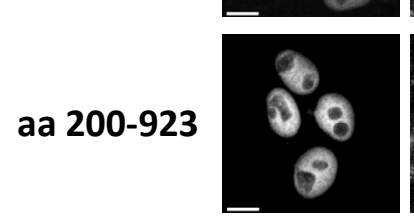

aa $220-923$
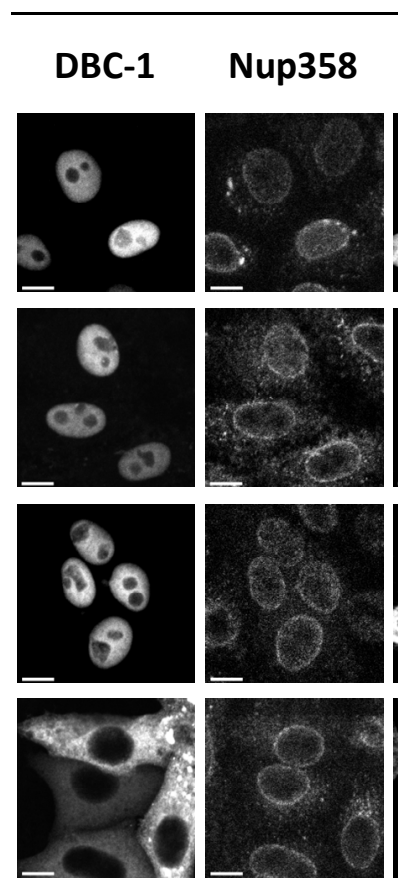

siRNA Nup358
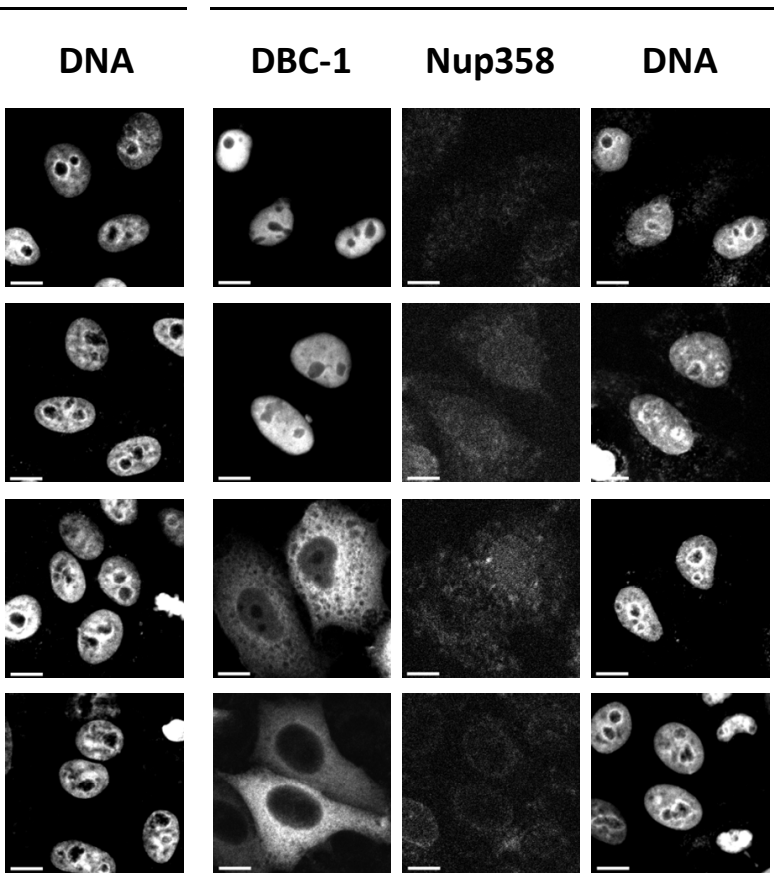

B

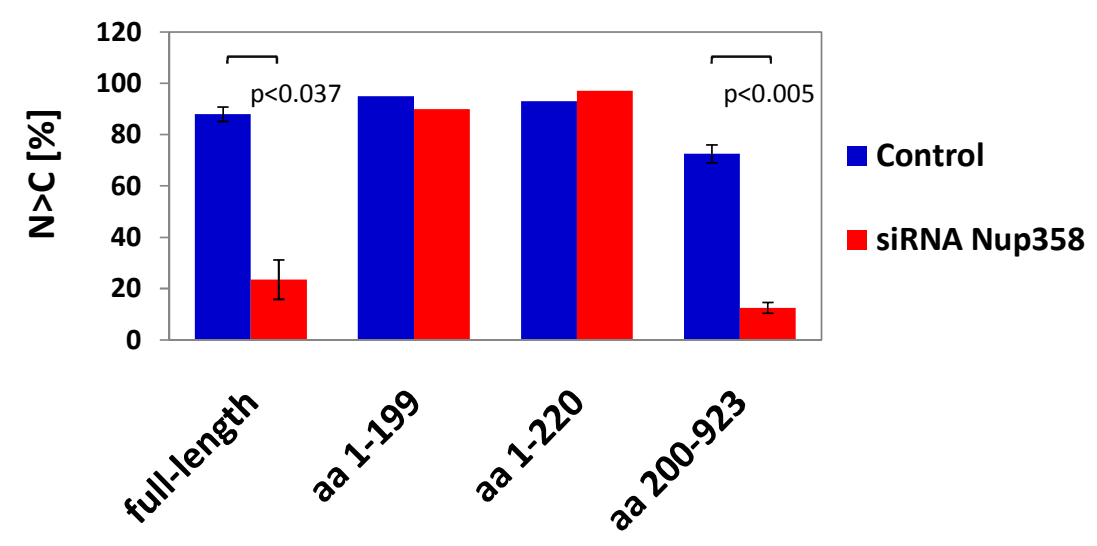

Figure 3-12: The C-terminal part of DBC-1 mediates Nup358 dependency.

A: Control or Nup358-depleted HeLa P4 cells were transiently transfected with plasmids coding for GFP $_{2}$-DBC-1 aa 1-199, GFP 2 -DBC-1 aa 1-220, GFP-DBC-1 aa 200-923 and GFP-DBC-1 aa 220-923. Nup358 was detected with a specific antibody, and DNA was visualized by Hoechst. Localization of the proteins was analyzed by fluorescence microscopy. Scale bars: $10 \mu \mathrm{m}$. B: For quantification, $>100$ cells were analyzed for the distribution of the fragments $\mathrm{GFP}_{2}$-DBC-1 aa 1-199, GFP 2 -DBC-1 aa 1-220, GFP-DBC-1 aa 200-923 and grouped into $\mathrm{N}>\mathrm{C}, \mathrm{N}=\mathrm{C}$ and $\mathrm{C}>\mathrm{N}$ (only $\mathrm{N}>\mathrm{C}$ is shown here). GFP-DBC-1 full-length served as positive control for the knockdown efficiency of Nup358. Error bars depict the standard deviation from the mean of three independent experiments. The statistical significance of the data was determined using the student's t-test. 


\subsubsection{The coiled-coil domain of DBC-1 is required for Nup358-dependent nuclear localization}

Having defined the nuclear import pathway of DBC-1, it was interesting to find out which region within DBC-1 mediates the Nup358 dependency of its nuclear localization. To identify a motif within the $\mathrm{N}$-terminal or $\mathrm{C}$-terminal part of DBC-1 which is responsible to mediate Nup358 dependency, we transfected the DBC-1 fragments into Nup358-depleted cells and compared their localization with control cells. The fragment aa 1-199 localized to the nucleus in control cells as well as in cells lacking Nup358 similar to fragment aa 1-220 (Figure 3-12 A). By contrast, the long C-terminal fragment, aa 200-923, containing the NLS accumulated into the cytoplasm upon depletion of Nup358. As expected, the C-terminal fragment without NLS stayed cytoplasmic in control as well as in Nup358 knockdown cells. The quantification of three independent experiments is illustrated in Figure 3-12 B. For unknown reasons, nuclear import of DBC-1 aa 200-923 was slightly less efficient in control cells with $72 \%$ compared to $88 \%$ for the full-length protein. Strikingly, this number decreased to $12 \%$ in cells lacking Nup358. Hence the $\mathrm{N}$-terminal region which includes the NLS does not mediate Nup358 dependency, but some domain/region within amino acids 200-923.

As the C-terminus of DBC-1 seemed to contain some sequence responsible for the Nup358 dependency, certain regions within the C-terminal part of DBC-1 were deleted, and analyzed for their subcellular localization in control as well as in cells depleted of Nup358. The selected regions referred to the postulated domains of the PROSITE and BLAST protein homology searches (Sundararajan et al., 2005). They reported a leucine zipper motif at amino acid 243-264 which interacts with Sirt1 (Kim et al., 2008). Furthermore, the sequence includes an EF-hand (calmodulin-like calcium binding region) at aa 704-748 and a coiled-coil domain aa 794-918 at its C-terminus (Figure 3-13 A).

After transient transfection, the deletion mutant lacking the leucine zipper, GFP-DBC-1 $\Delta$ aa 243-264, was not imported as efficiently as DBC-1 wild type as some cells displayed a relatively high cytoplasmic pool of the mutant in control cells (Figure 3-13 B). However, this mutant exhibited the same behavior as wild type DBC-1 and accumulated in the cytoplasm of Nup358-depleted cells. 
A

full-length

$\Delta$ aa 243-264

$\Delta$ aa $704-748$

aa 1-793

B

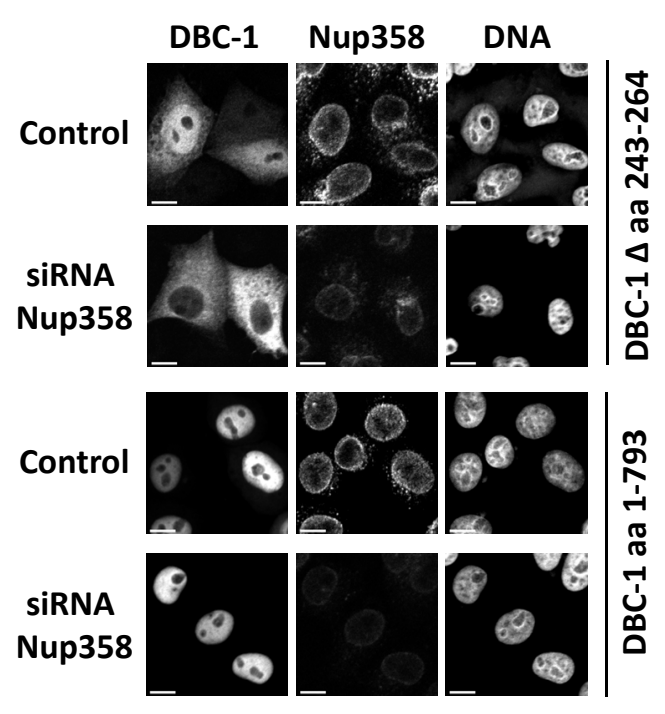

C

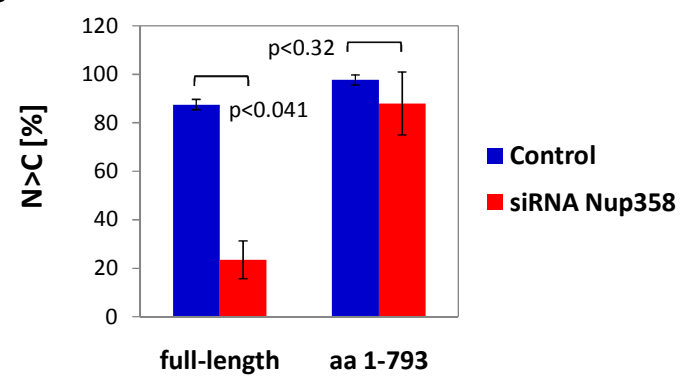

E

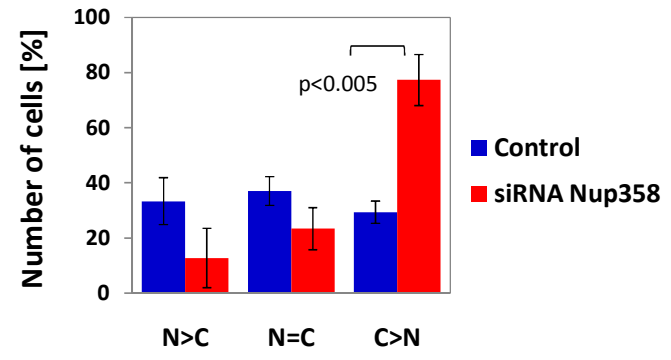

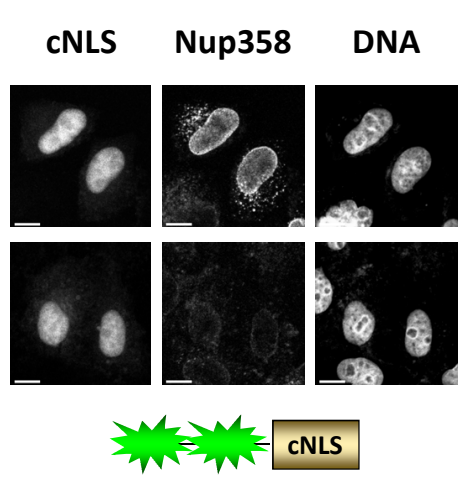

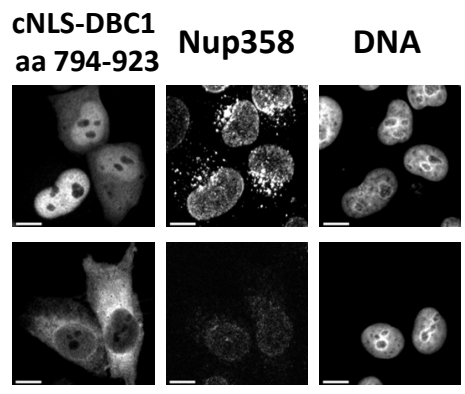

Nup358

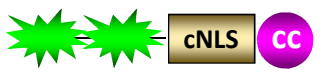

Localization Nup358

in mock dependent import

N yes

Nyes

Nyes

N no

D 
Likewise, GFP-DBC-1 $\Delta$ aa 704-748, lacking the EF-hand, showed the same Nup358 dependency as wild type (data not shown). Interestingly, the mutant GFP-DBC-1 aa 1-793 lacking the coiled-coil region localized to the nucleus in control cells as well as in Nup358-depleted cells (Figure 3-13 B). The mutant localized to the nucleus in $97 \%$ of the cells which did not change after depletion of Nup358 (Figure 3-13 C).

We next tested whether it was possible to transfer the Nup358 dependency from DBC-1 to any other protein like $\mathrm{GFP}_{2}$-cNLS because nuclear localization of $\mathrm{GFP}_{2}$-cNLS does not depend on Nup358 (Hutten et al., 2006) (Figure 3-13 D). Hence, the coiled-coil domain of DBC-1, aa 794-923, was fused in frame to $\mathrm{GFP}_{2}-\mathrm{CNLS}$, and the localization was analyzed in control and Nup358-depleted cells. The fusion protein localized to the nucleus in $34 \%$ of the cells. $37 \%$ of cells showed equal distribution of the reporter and $29 \%$ were cytoplasmic (Figure 3-13 E). Surprisingly, the fusion of the coiled-coil domain to $\mathrm{GFP}_{2}$-cNLS resulted in impaired import of the reporter protein in Nup358-depleted cells. The number of cells displaying cytoplasmic localization increased significantly from $29 \%$ to $77 \%$.

In summary, the coiled-coil region of DBC-1, aa 794-923, seems to be involved in the Nup358 dependency of DBC-1 because a mutant lacking this domain could be imported into the nucleus in cells depleted of Nup358 in contrast to wild type DBC-1. Furthermore, the Nup358 dependency could be transferred to a reporter which import did not depend on Nup358. This suggests involvement of the coiled-coil domain of DBC-1 in nuclear export comparable to the shuttling-behavior of the reporter protein NES-GFP 2 -cNLS; the exact mechanism needs further analysis.

Figure 3-13: The coiled-coil domain of DBC-1 is required for Nup358-dependent nuclear localization.

A schematic description of the deletion mutants of DBC-1 are shown in A, including their subcellular localization ( $\mathrm{N}=$ nucleus) in control cells. Further, Nup358-dependent nuclear import is indicated.

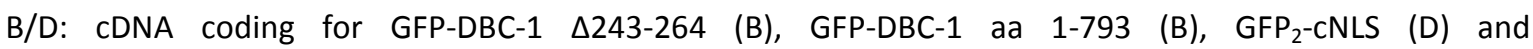
GFP $_{2}$-CNLS-DBC-1 aa 794-923 (D) were transiently transfected in control or siRNA-treated HeLa P4 cells. After immunostaining of Nup358 and detection of the DNA with Hoechst, the localization of the proteins was determined by fluorescence microscopy. C/E: In the quantification, > 100 cells were analyzed concerning the cellular distribution $\mathrm{N}>\mathrm{C}, \mathrm{N}=\mathrm{C}$ and $\mathrm{C}>\mathrm{N}$ of GFP-DBC-1 aa 1-793 (C) and GFP $_{2}$-CNLS-DBC-1 aa 794-923 (E). GFP-DBC-1 served as a positive control for the knock-down efficiency. Error bars depict the standard deviation from the mean of three independent experiments. The statistical significance of the data was determined using the student's t-test. 


\subsection{The role of Nup358 in nuclear import of certain substrates}

After characterization of the import mechanism of the Nup358-dependent substrates, the next section will focus on Nup358. In particular, it should be characterized which domains of the nucleoporin are responsible for the cytoplasmic accumulation of specific substrates in cells depleted of Nup358.

First, an exogenous full-length Nup358 should rescue the import defect of substrates like DBC-1 in Nup358-depleted cells. Second, truncation mutants of Nup358 must be tested in their rescue ability to disclose to the domains of Nup358 mediating nuclear import of the proteins.

\subsubsection{Exogenous Nup358 localizes to the nuclear pore}

Starting point of the rescue experiment with Nup358 truncations was the cloning of an RNAi-resistant exogenous full-length Nup358 serving as positive control for the rescue of the import defect. Such rescue experiments present the ultimate control for RNAi treatments and validate the specificity of the observed effects. Another specificity control would be the use of other target sequences for the siRNA which resulted in a similar phenotype (Hutten et al., 2009).

Thus, we introduced two silent third-codon point mutations (wobble-mutations) within the target region of the siRNA corresponding to amino acids 117-123. Further, an HA- or an mRFP-tag was introduced N-terminally of the Nup358 sequence for detection of the exogenous protein (Figure 3-14 A).

Hence, localization and functionality of the RNAi-resistant HA-Nup358 were confirmed. HeLa P4 cells were transfected with HA-Nup358 which was detected with an $\alpha$-HA-antibody. Further, endogenous and exogenous Nup358 and Nup214 were detected with corresponding antibodies. Exogenous Nup358 localized like the endogenous protein to the NPC (Figure 3-14 B). Clearly, it did not affect its endogenous counterpart. Localization of Nup214, another cytoplasmic nucleoporin, did not change localization due to transfected full-length HA-Nup358. 


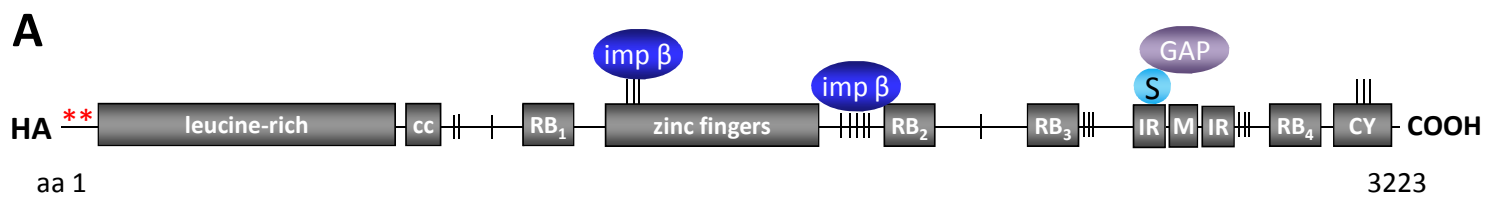

B

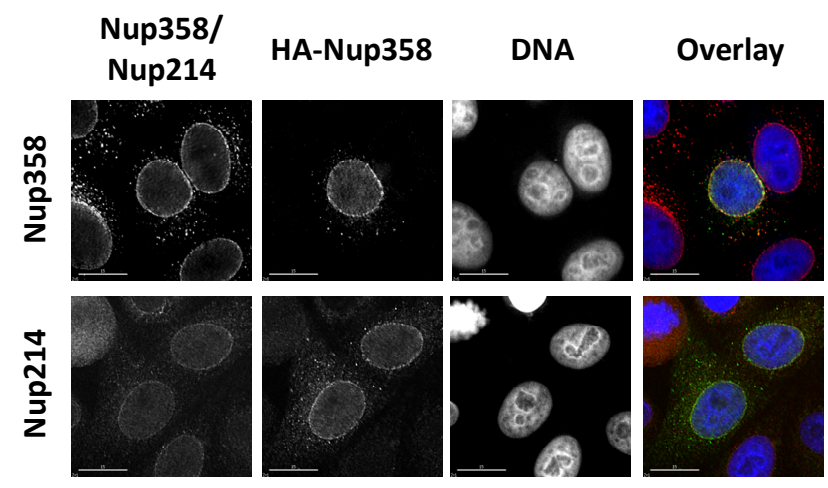

D
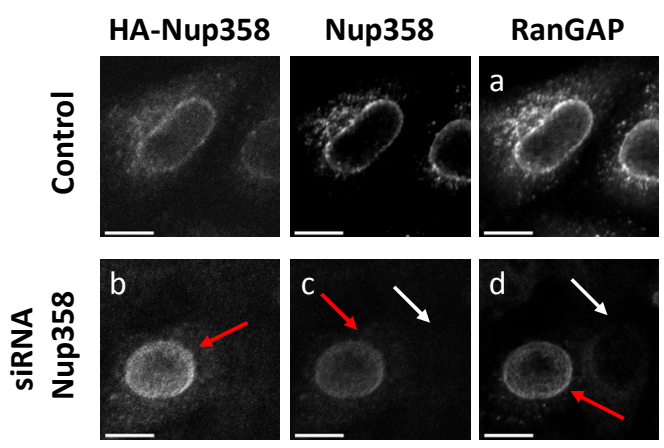

DNA
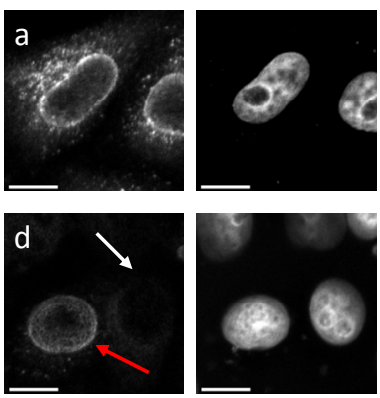

C
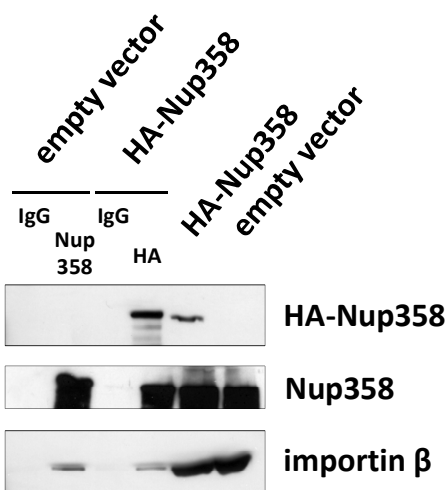

Figure 3-14: Exogenous HA-Nup358 behaves like the endogenous protein with respect to NPC-localization and interaction with several proteins.

A: Scheme of HA-tagged Nup358 with possible interaction sites for importins (imp $\beta$ ) and sumoylated RanGAP1. FG-repeats are shown as dashes. Red asterisks indicate the silent mutation for siRNA resistance. B: A plasmid coding for HA-Nup358 was transfected in HeLa P4 cells. Endogenous Nup358 and Nup214 were detected with respective antibodies. HA-Nup358 was stained with an $\alpha$-HA-antibody and was also recognized by the $\alpha$-Nup358 antibody. DNA was visualized by Hoechst. Images were taken using a Delta vision microscope. Afterwards, deconvolution of the imaged z-stacks was performed. Scale bars: $15 \mu \mathrm{m}$.

C: Immunoprecipitations were performed in HeLa P4 cells using an $\alpha$-HA-antibody for cells transfected with HA-Nup358 or an $\alpha$-Nup358 antibody for cells transfected with an empty vector. IgG served as specificity control. Bound proteins and possible interaction partners were separated by SDS-PAGE, followed by western blotting using specific antibodies against HA, Nup358, importin $\beta$ and RanGAP1. The modification of RanGAP1 with SUMO1 results in a $20 \mathrm{kDa}$ size shift in SDS-PAGE, indicated as RanGAP1*S1. The input corresponds to $1 \%$ of total lysate. D: Control or Nup358-depleted HeLa P4 cells were transfected with an siRNA-resistant mutant of HA-Nup358. To localize the proteins, $\alpha-H A-, \alpha-N u p 358-$ and $\alpha$-RanGAP1 antibodies were used, respectively. Image $c / d$ : white arrow shows a cell depleted of Nup358 which leads to a loss of NPC-associated RanGAP1. The presence of HA-Nup358 (image b, red arrow) rescued the localization of RanGAP1 to the nuclear pore (image d, red arrow). 
After confirmation of the proper localization of HA-Nup358, the expression of HA-Nup358 as full-length protein and binding of two interaction partners were examined. For that purpose, immunoprecipitations were carried out with either an $\alpha$-HA-antibody in HA-Nup358 transfected cells or an $\alpha$-Nup358 antibody for cells transfected with an empty vector, respectively. Rabbit IgG served as specificity control in both cases. HA-Nup358 could be enriched in the immunoprecipitation compared to the total lysate (Figure 3-14 B, HA-blot). Detection with an $\alpha$-Nup358 antibody revealed precipitated endogenous Nup358 and exogenous HA-Nup358 having the same size.

Importin $\beta$ and sumoylated RanGAP1 are two well-established interacting proteins for Nup358 (Delphin et al., 1997; Matunis et al., 1998) (Figure 3-14 A). To test their binding to HA-Nup358, we examied the immunoprecipitated complex for these two proteins. As shown in Figure 3-14 C, endogenous as well as exogenous Nup358 interacted with importin $\beta$. The modification of proteins with SUMO results in a $20 \mathrm{kDa}$ size shift in SDS-PAGE. The Sumo-modified version of RanGAP1 (RanGAP1*S1) strongly interacted with endogenous as well as exogenous Nup358. A small amount of unsumoylated RanGAP1 interacted unspecifically with IgG-coupled protein-A agarose.

As a result of the interaction with the nuclear pore, the RanGAP1 stain in control cells revealed the typical rim stain (Figure 3-14 D, image a). Depletion of Nup358 (image c, white arrow) was shown to lead to a loss of NPC-associated RanGAP1 (Hutten et al., 2008) (image d, white arrow). Transfection of HA-Nup358 into knockdown cells (image b, red arrow) could rescue the localization of RanGAP1 to the nuclear pore (image $d$, red arrow). Together, exogenous HA-Nup358 behaves like the endogenous protein with respect to NPC-localization and interaction with several proteins. 


\subsubsection{Exogenous Nup358 can rescue the import defect of DBC-1}

Given that the exogenous HA-Nup358 behaves like its endogenous counterpart, the ability to rescue the import defect of DBC-1 in cells depleted of Nup358 was tested. Thus, HA-Nup358, whose mRNA should not be degraded by the RNAi, was cotransfected with GFP-DBC-1 in cells depleted of Nup358. For analysis, the exogenic protein was detected with an $\alpha$-HA-antibody, endogenous and transfected with an $\alpha$-Nup358 antibody. Cells positive for both, HA-Nup358 and GFP-DBC-1 were analyzed. As the $\alpha$-Nup358 antibody also detected the HA-Nup358 protein thereby masking an efficient knockdown, we characterized only cells whose bystanders clearly lacked Nup358, suggesting an efficiently transfected area of cells. Mostly all cells in a certain area were depleted of Nup358. To further ensure an efficient depletion of Nup358, the location of DBC-1 was analyzed in control and Nup358-depleted cells that were not transfected with HA-Nup358, but coming from the same siRNA transfection.

In subsequent experiments, the $\alpha$-Nup358 antibody did not detect the Nup358 truncations as its epitope is the very C-terminal cyclophilin-like domain, so only endogenous and exogenous full-length Nup358 were recognized.

Strikingly, cotransfection of HA-Nup358 was indeed able to rescue the import defect of DBC-1 in Nup358-depleted cells (Figure 3-15 A). The number of cells with DBC-1 in the nucleus increased significantly from $33 \%$ to $70 \%$ in presence of HA-Nup358 (Figure 3-15 B). Furthermore, the rescue experiment was also carried out with mRFP-Nup358, which localized to the nuclear pore as well. In presence of mRFP-Nup358, DBC-1 could be imported even more efficiently compared to HA-Nup358 (Figure 3-15 A), and the number of cells with DBC-1 in the nucleus increased significantly from $33 \%$ to $90 \%$ (Figure 3-15 B) which demonstrates that the mRFP-Nup358 was functional as well. In conclusion, the rescue experiment validated the specificity of the observed cytoplasmic accumulation of the proteins in Nup358-depleted cells, and points to a specific role of Nup358 in mediating nuclear transport of DBC-1. 
A

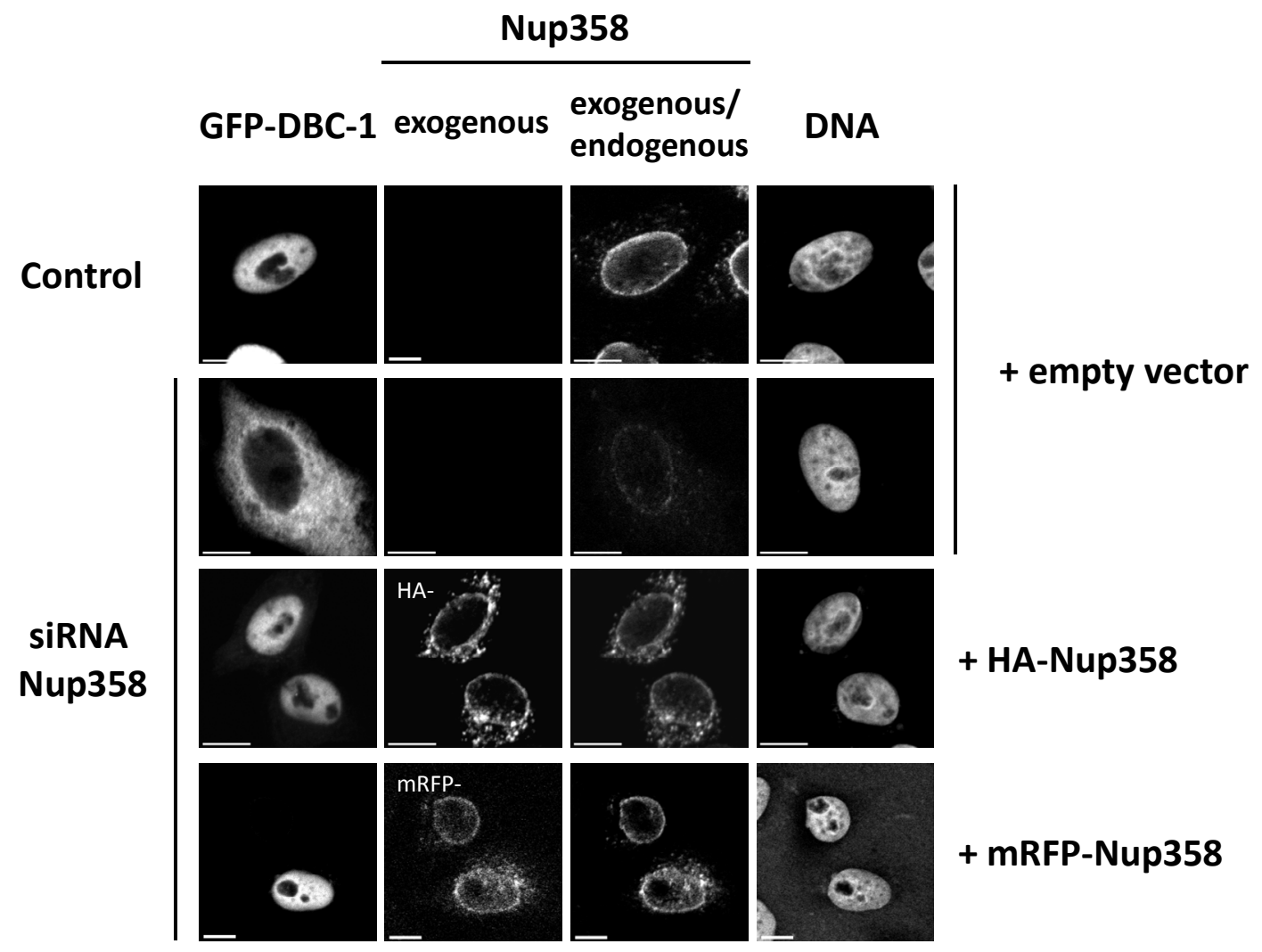

B

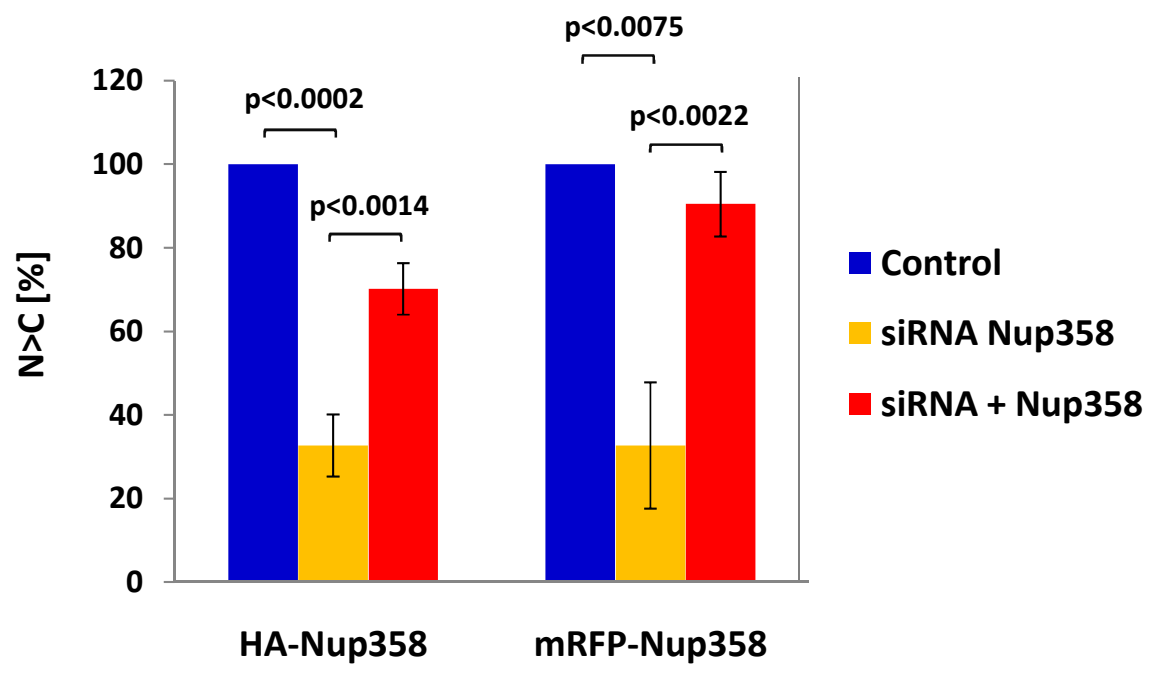




\subsection{3 siRNA-resistant truncation mutants of Nup358}

Having this very powerful tool of exogenous HA-Nup358 to rescue DBC-1 in Nup358-depleted cells, we deleted single domains in order to identify the region mediating the import for DBC-1 (Figure 3-16 A). These HA-tagged truncation mutants were RNAi-insensitive as well. First, their expression and binding of importin $\beta$ and RanGAP1 were analyzed in coimmunoprecipitations as performed with full-length HA-Nup358. Even though the expression levels of the different truncations varied in correlation with the size of the expressed proteins (Figure 3-16 B, total lysate), most fragments could be enriched by precipitation with an $\alpha$-HA-antibody. Only fragment aa 1-3047, lacking the cyclophilin-like domain, could not be precipitated efficiently, but it had the correct size as seen in the total lysate.

The amount of bound importin $\beta$ decreased with the size of the truncations which is in accordance with the declining number of FG-repeats in the shorter fragments (importin $\beta$ blot, refer to Figure 3-16 A for number of FG-repeats). RanGAP1 binds to Nup358 between amino acid 2622-2645 (Song et al., 2004). This explains why only full-length and fragment aa 1-2684 could precipitate RanGAP1, the latter less efficiently (Figure 3-16 B). These results indicate the functionality of the fragments as they were expressed corresponding to their estimated size and could bind importin $\beta$. RanGAP1 did not bind to fragments lacking the interaction site for the protein as expected.

Figure 3-15: Exogenous Nup358 can rescue nuclear import of DBC-1 in cells lacking endogenous Nup358. A: Control or Nup358-depleted HeLa P4 cells were cotransfected with plasmids coding for GFP-DBC-1 and either an empty vector or siRNA-resistant mutants of HA-Nup358 and mRFP-Nup358, as indicated. For analysis, transfected HA-Nup358 was detected with an $\alpha$-HA-antibody, endogenous and transfected (HA- as well as mRFP-version) with an $\alpha$-Nup358 antibody. DNA was visualized by Hoechst. Scale bars: $10 \mu \mathrm{m}$. B: For quantification, distribution of GFP-DBC-1 was analyzed in the absence or presence of exogenous HA- or mRFP-Nup358, and grouped into the three categories $\mathrm{N}>\mathrm{C}, \mathrm{N}=\mathrm{C}$ or $\mathrm{C}>\mathrm{N}$ (only $\mathrm{N}>\mathrm{C}$ is shown here). As the $\alpha$-Nup358 antibody also detected exogenous Nup358 thereby masking an efficient depletion, only cells were characterized whose bystanders clearly lacked Nup358, suggesting an efficiently transfected area of cells. For a better comparison, the number of mock cells in the category $\mathrm{N}>\mathrm{C}$ was set to $100 \%$. The localization in knock-down cells with or without overexpression of the Nup358 truncations was calculated in relation to the distribution in mock cells. Error bars indicate the standard deviation from the mean of three independent experiments with > 100 cells analyzed in each single experiment. The statistical significance of the data was determined using the student's t-test. 
A

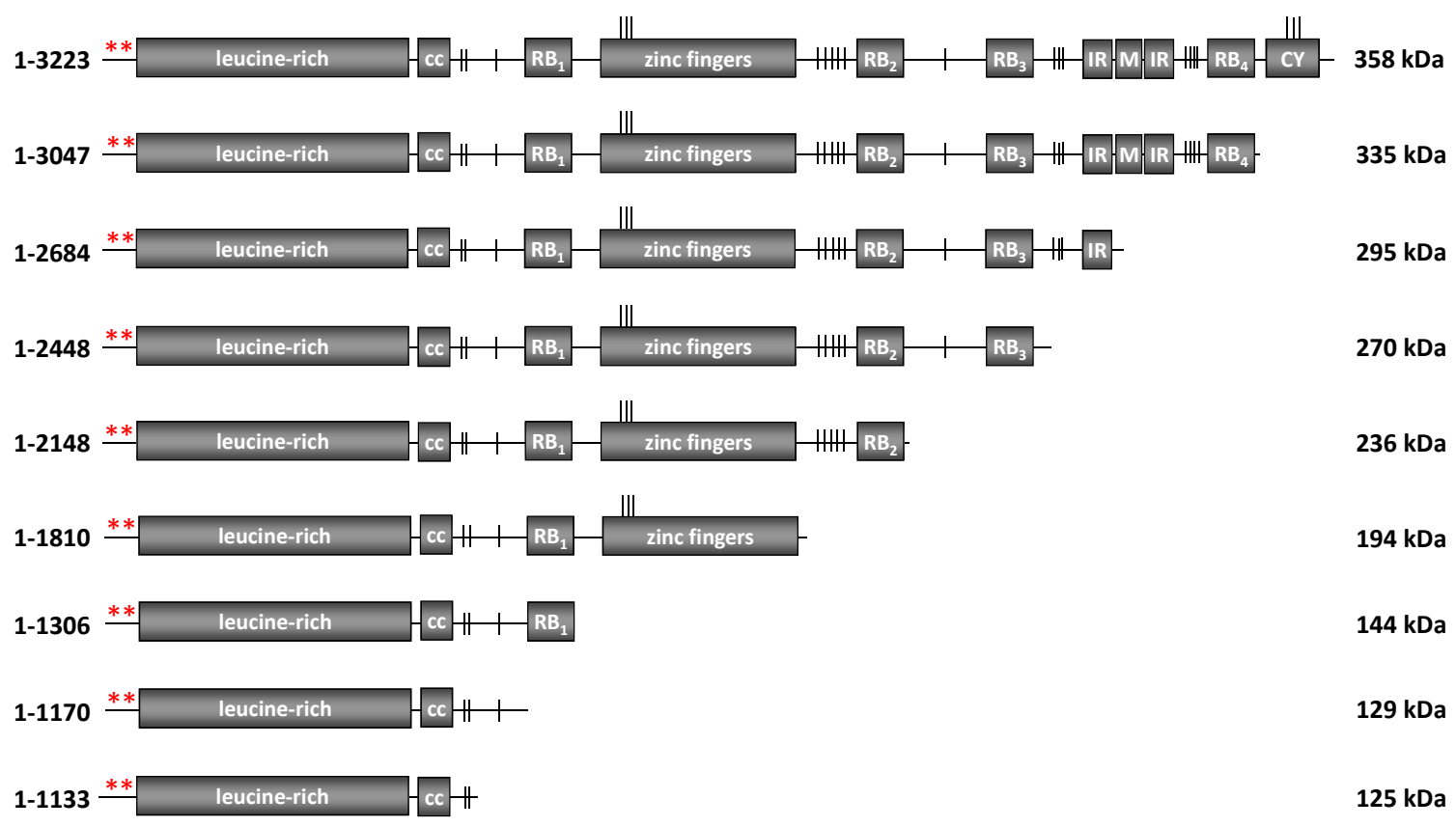

B

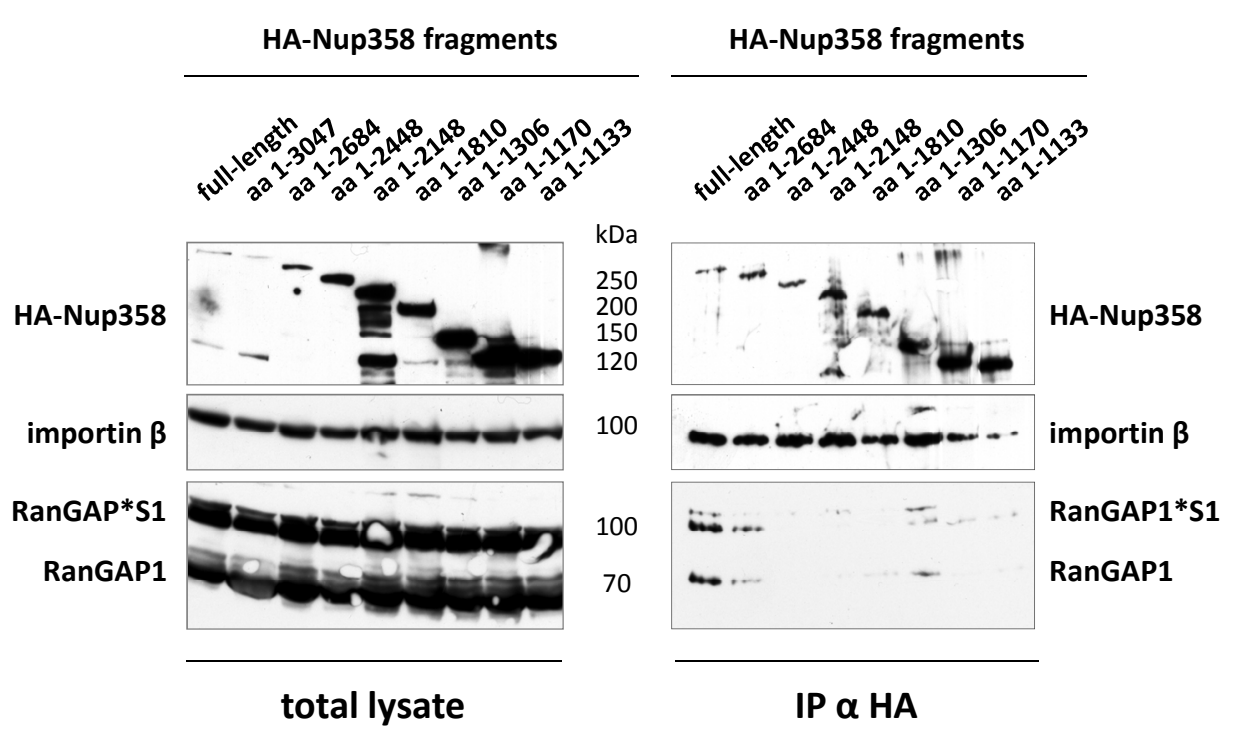

Figure 3-16: siRNA-resistant truncations of Nup358 can bind importin $\beta$ and RanGAP1, respectively.

A: All siRNA-resistant truncations of Nup358 which were analyzed for the rescue ability of Nup358-dependent import are shown here, including their molecular weigths. The proteins contain an HA-tag. B: HeLa P4 cells were transfected with plasmids coding for the HA-Nup358 truncations, and immunoprecipitations using $\alpha-H A$ antibodies were performed. Only fragment aa 1-3047, lacking the cyclophilin-like domain, could not be precipitated efficiently. Bound proteins were separated by SDS-PAGE and detected by western blotting with $\alpha$-HA, $\alpha$-importin $\beta$ and $\alpha$-RanGAP1 antibodies. The modification of RanGAP1 with SUMO1 results in a $20 \mathrm{kDa}$ size shift in SDS-PAGE, indicated as RanGAP1*S1. The input corresponds to $1 \%$ of total lysate. 
As fragment aa 1-1133 is sufficient for incorporation into the nuclear pore, all fragments displayed proper localization (see also Figure 3-18 A), suggesting that the $\mathrm{N}$-terminal part of Nup358 contains a sequence for incorporation into the NPC.

To further narrow down the region/motif required for NPC-association, we transfected HA-tagged Nup358 fragments into HeLa P4 cells, and determined their localization. Nup358 aa 1-1133 spanning the leucine-rich and the coiled-coil domain, localized to the NPC as its accumulation showed the characteristic rim stain (Figure 3-17, image a). A fragment containing the leucine-rich region, aa 1-805, accumulated to the cytoplasm (image b). The coiled-coil domain, aa 806-1133, was not sufficient either to localize to the pore (image c), and accumulated in the nucleus and cytoplasm.

Hence, amino acids 1-1133 display the anchor sequence of Nup358 which is sufficient and necessary to localize the nucleoporin to the pore. During the course of this work, similar results have been published by Joseph and coworkers (Joseph et al., 2008). It remains still unclear, how in particular Nup358 is anchored to the NPC.

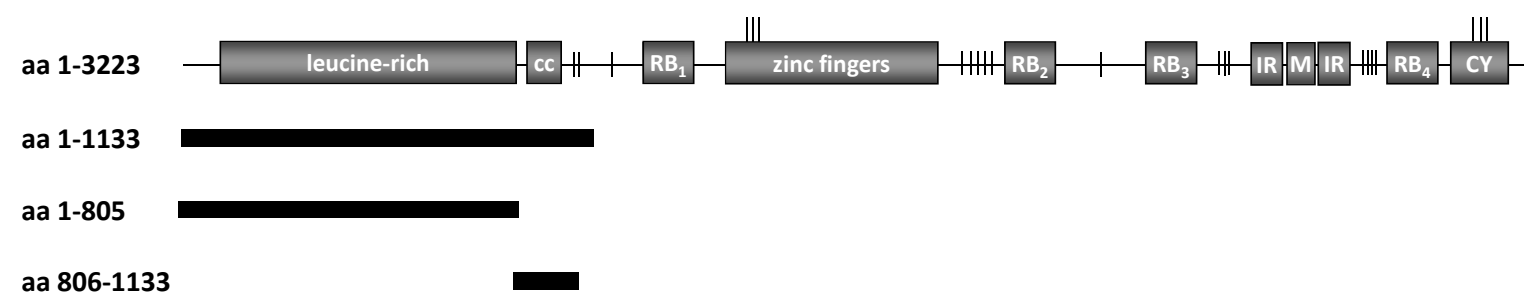

HA-Nup358 fragments

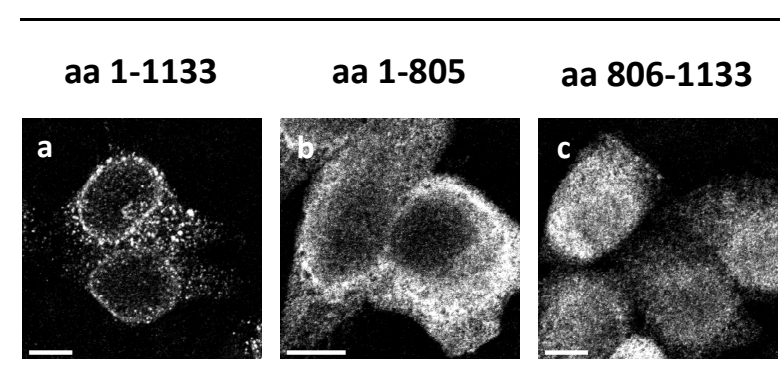

Figure 3-17: The leucine-rich region and the coiled-coil domain of Nup358 are required for NPC-anchorage HeLa P4 cells were transfected with plasmids coding for the indicated HA-labeled Nup358 fragments. An $\alpha-\mathrm{HA}$-antibody was used to detect the expressed proteins. Scale bars: $10 \mu \mathrm{m}$. 
A
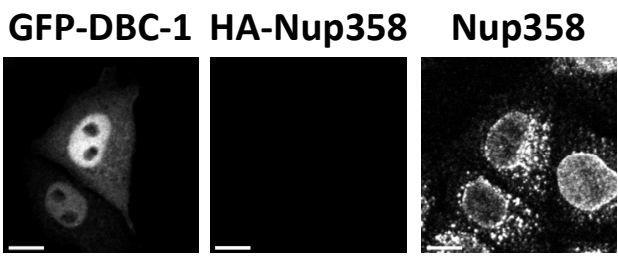

DNA

Control
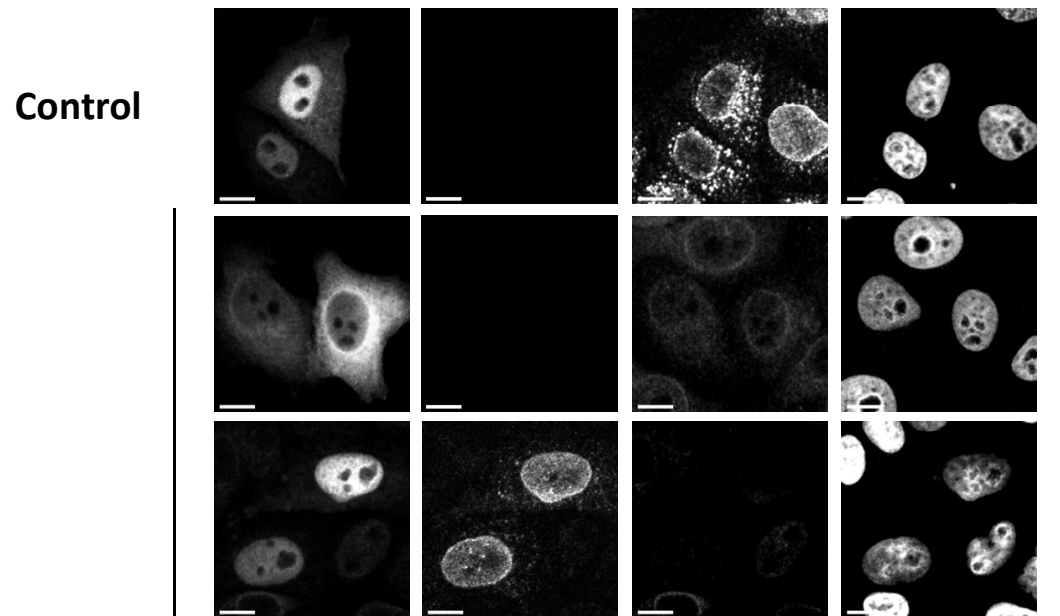

+ empty vector
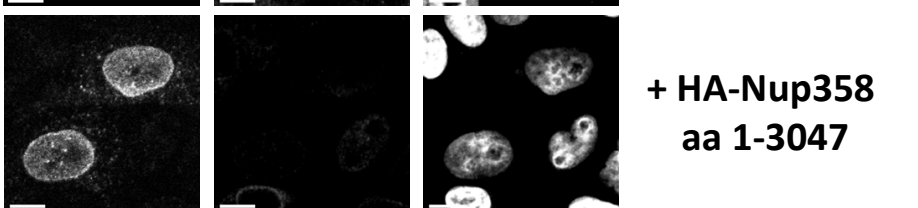

SiRNA
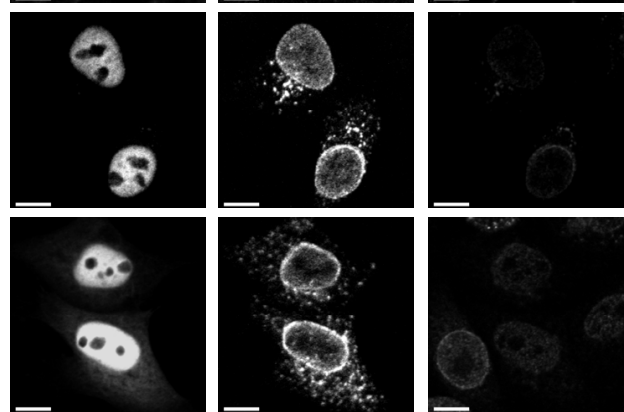

5 aa 1-3047

Nup358
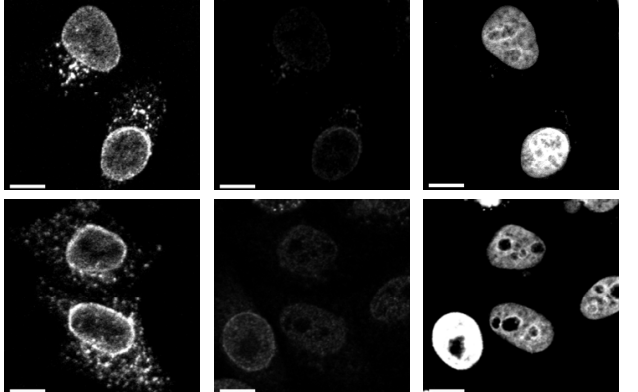

+ HA-Nup358

aa 1-2448
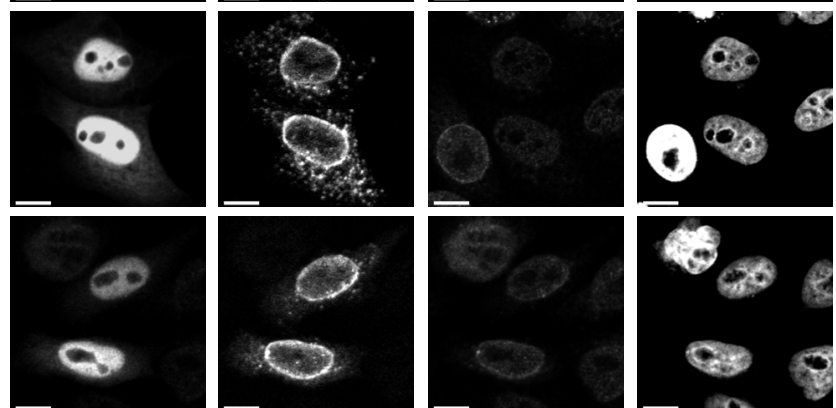

+ HA-Nup358

aa 1-2148

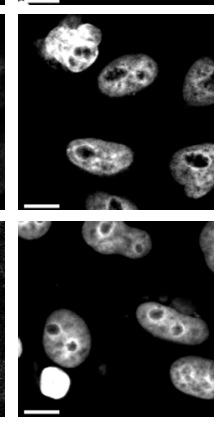

+ HA-Nup358

aa 1-1306
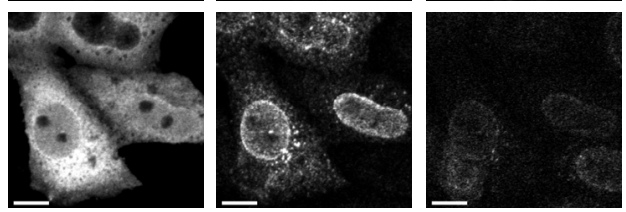

\section{+ HA-Nup358}

aa 1-1133

B

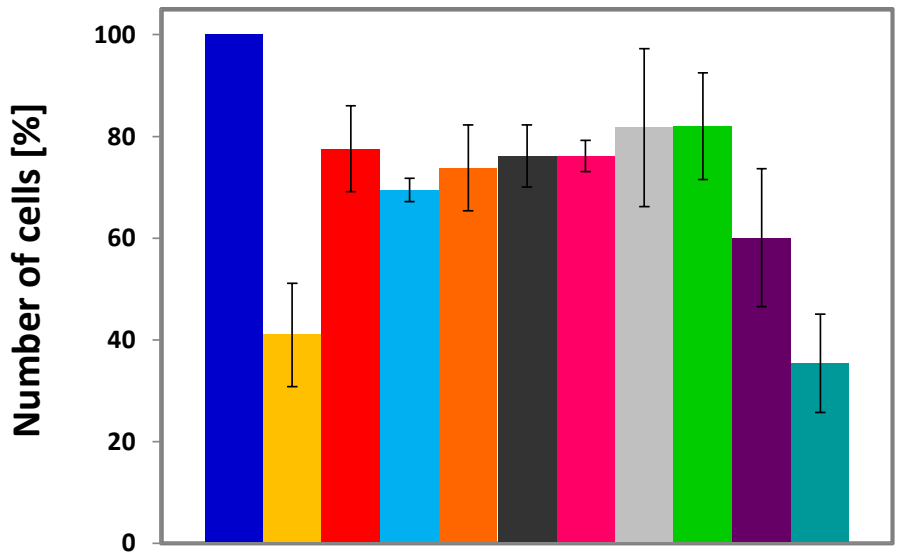

- Control

siRNA Nup358

- siRNA + HA-Nup358

- siRNA + HA-Nup358 aa 1-3047

- siRNA + HA-Nup358 aa 1-2684

- siRNA + HA-Nup358 aa 1-2448

- siRNA + HA-Nup358 aa 1-2148

siRNA + HA-Nup358 aa 1-1810

- siRNA + HA-Nup358 aa 1-1306

- siRNA + HA-Nup358 aa 1-1170

siRNA + HA-Nup358 aa 1-1133

$\mathrm{N}>\mathrm{C}$ 


\subsubsection{The N-terminal part of Nup358 mediates nuclear import of DBC-1}

The rescue experiments with the different truncation mutants of Nup358 were carried out as illustrated with full-length HA-Nup358. Noteworthy, the antibody used here to detect endogenous Nup358 recognized an epitope at the very C-terminal cyclophilin-like domain of Nup358 which is missing in the truncations (see Figure 3-18 A). By this, only cells with clearly reduced Nup358 levels could be analyzed. Further, only similar expression patterns of the truncations were included in the quantification as especially the longer fragments had a lower expression levels compared to the shorter fragments. After depletion of Nup358, the number of cells with DBC-1 in the nucleus was significantly reduced from $100 \%$ to $41 \%$ as also shown before. Expression of a truncation lacking the cyclophilin-like domain, aa 1-3047, increased this number to $69 \%$ (Figure 3-18 A+B) (for p-values, see supplementary material, Figure IV). In the same way, other fragments of Nup358 could partially compensate for the lack of Nup358 like the truncations aa 1-2684 (74 \%) and aa 1-2448 (76 \%) (Figure 3-18 A+B).

This was interesting as the shorter fragment did not contain the RanGAP1 association site, and hence nuclear import could occur in presence of only soluble RanGAP1. A fragment containing two of the four Ran-binding domains (HA-Nup358 aa 1-2148) promoted nuclear import to $76 \%$. A truncation stopping after the zinc-finger domain, aa 1-1810, behaved similarly.

Figure 3-18: The $\mathrm{N}$-terminal third of Nup358 is sufficient to mediate nuclear import of DBC-1.

A: Control or Nup358-depleted HeLa P4 cells were cotransfected with plasmids coding for GFP-DBC-1 and either an empty vector or siRNA-resistant truncations of Nup358, as indicated. For analysis, the transfected HA-Nup358 truncations were detected with an $\alpha$-HA-antibody. The $\alpha$-Nup358 antibody detects an epitope at the very C-terminus of Nup358 which is missing in the truncations. By this, only cells with clearly reduced Nup358 levels were characterized. DNA was visualized by Hoechst. Localization of the proteins was analyzed by fluorescence microscopy. Scale bars: $10 \mu \mathrm{m}$. B: For quantification, distribution of GFP-DBC-1 in the absence or presence of the HA-Nup358 truncations was analyzed and grouped into the three categories $\mathrm{N}>\mathrm{C}, \mathrm{N}=\mathrm{C}$ or $\mathrm{C}>\mathrm{N}$ (only $\mathrm{N}>\mathrm{C}$ is shown here). For a better comparison, the number of control cells in the category $\mathrm{N}>\mathrm{C}$ was set to $100 \%$. The localization in knockdown cells with or without overexpression of the Nup358 truncations was calculated in relation to the distribution in control cells. Error bars indicate the standard deviation from the mean of three independent experiments with $>100$ cells analyzed in each single experiment. For p-values, refer to supplementary material, Figure IV. 
A

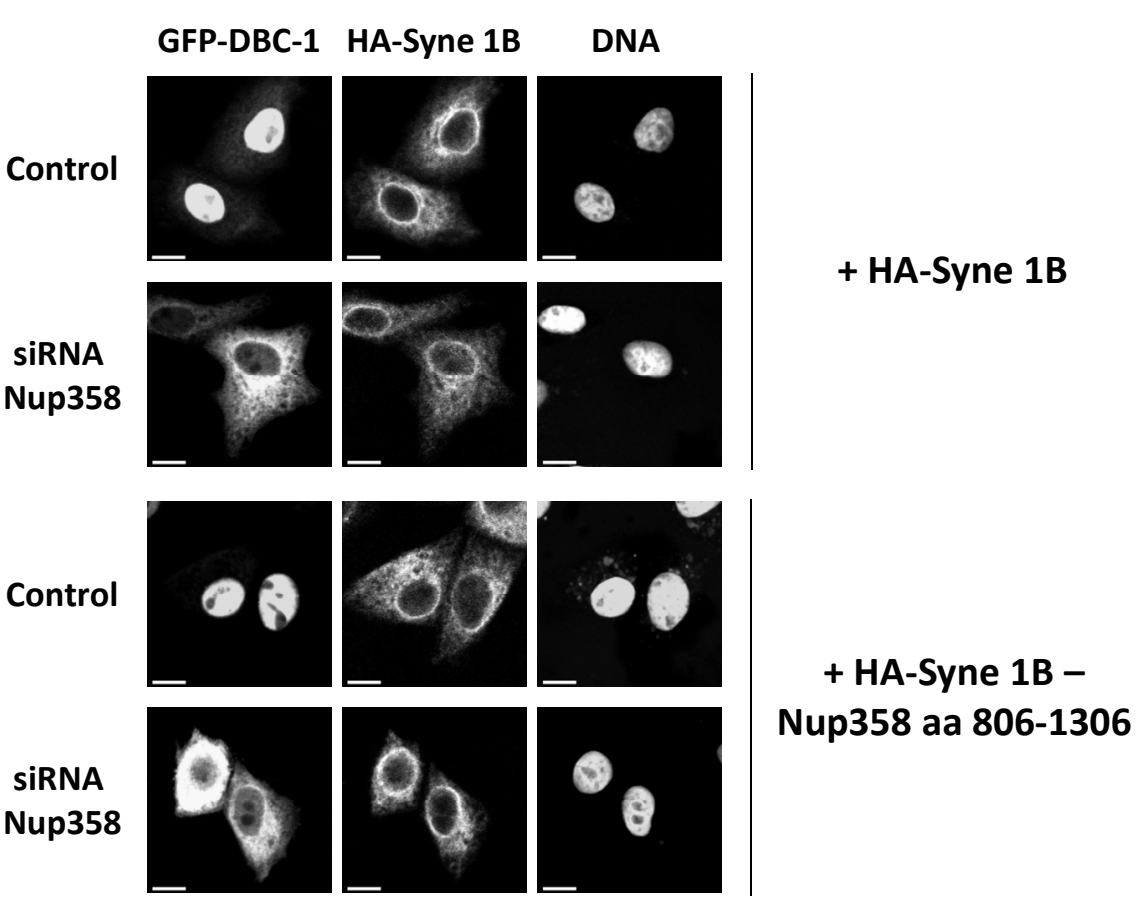

B

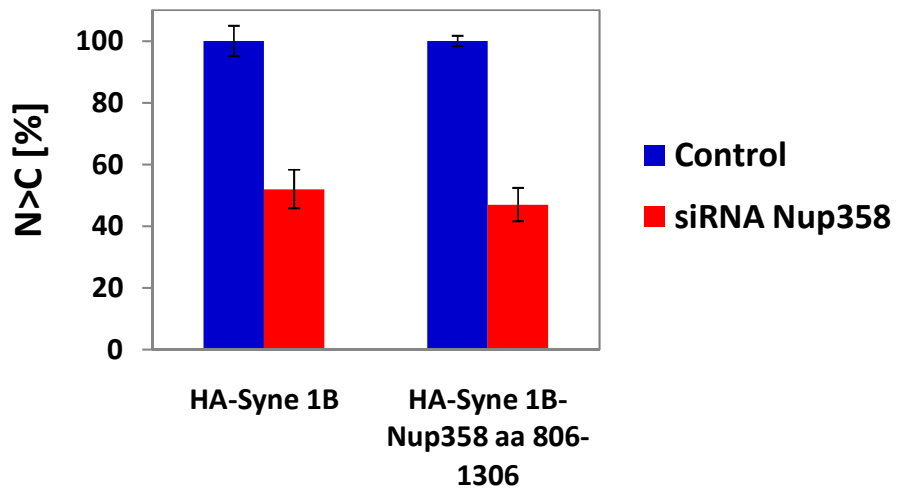

Figure 3-19: Incorporation into the nuclear pore is required to compensate for the loss of endogenous Nup358 to import DBC-1.

A: Control or Nup358-depleted HeLa P4 cells were cotransfected with plasmids coding for GFP-DBC-1 and HA-Syne 1B or HA-Syne 1B-Nup358 aa 806-1306, as indicated. For analysis, the transfected HA-constructs were detected with an $\alpha-H A$-antibody. DNA was visualized by Hoechst. Localization of the proteins was analyzed by fluorescence microscopy. Scale bars: $10 \mu \mathrm{m}$. B: For quantification, distribution of GFP-DBC-1 was analyzed and grouped into the three categories $\mathrm{N}>\mathrm{C}, \mathrm{N}=\mathrm{C}$ or $\mathrm{C}>\mathrm{N}$ (only $\mathrm{N}>\mathrm{C}$ is shown here). For a better comparison, the number of control cells in the category $\mathrm{N}>\mathrm{C}$ was set to $100 \%$. The localization in knockdown cells was calculated in relation to the distribution in control cells. Error bars indicate the standard deviation from the mean of three independent experiments with $>100$ cells analyzed in each single experiment. To ensure an efficient depletion of Nup358, the location of DBC-1 was analyzed in Nup358-depleted cells which were not transfected with the HA-Syne 1B fusions, but coming from the same siRNA transfection (data not shown). 
The fragment with one RanBD, aa 1-1360, could increase the number of DBC-1 accumulated into the nucleus to $82 \%$. A shorter fragment just lacking the RanBD, aa $1-1170$, resulted only in $60 \%$ nuclear location of DBC-1. Interestingly, a fragment lacking 30 additional amino acids, aa 1-1133, completely failed to compensate for the lack of Nup358. Here, the number of cells with accumulation of DBC-1 in the nucleus did not differ from cells depleted of Nup358 (Figure 3-18 A+B).

In summary, the N-terminal part of Nup358 containing only one RanBD was sufficient to promote nuclear import of DBC-1 in cells lacking endogenous Nup358. Hence, pore-associated RanGAP1 was not required. A fragment without any RanBD was slightly less efficient, but still able to promote nuclear import suggesting that soluble RanBP1 can compensate for the loss of Nup358's Ran-binding domains.

\subsubsection{Incorporation into the nuclear pore is required to compensate for the loss of endogenous Nup358 to import DBC-1}

After identification of a region of Nup358 mediating nuclear import of DBC-1, an interesting aspect was if this region had to be an integral part of the NPC or if localization to the outer nuclear membrane might be sufficient to promote nuclear import of DBC-1. Mammalian Syne 1 and 2 (also Nesprin, NUANCE, myne) are proteins containing a

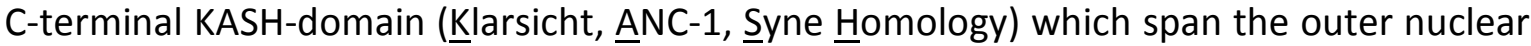
membrane (reviewed in Starr, 2007). Hence, Nup358 aa 806-1306 was fused in frame to the last 350 amino acids of murine Syne 1B, aa 1639-1989, (HA-Syne 1B) which are sufficient for incorporation into the outer nuclear membrane (Grady et al., 2005). Hence, it was able to recruit the Nup358 fragment to the outer nuclear membrane in wild type as well as in Nup358-depleted cells (Figure 3-19 A).

In order to analyze the rescue ability, either HA-Syne 1B or HA-Syne 1B-Nup358 aa 806-1306 was cotransfected with DBC-1 in Nup358-depleted cells. Neither the KASH-domain alone nor the fusion with the Nup358 fragment promoted nuclear import of DBC-1 (Figure 3-19 A+B). The number of cells which were depleted of Nup358 and had DBC-1 in the nucleus did not increase in presence of the fusion protein HA-Syne 1B-Nup358 aa 806-1306 (47 \%) compared to HA-Syne 1B (52 \%) (Figure 3-19 B). 
A

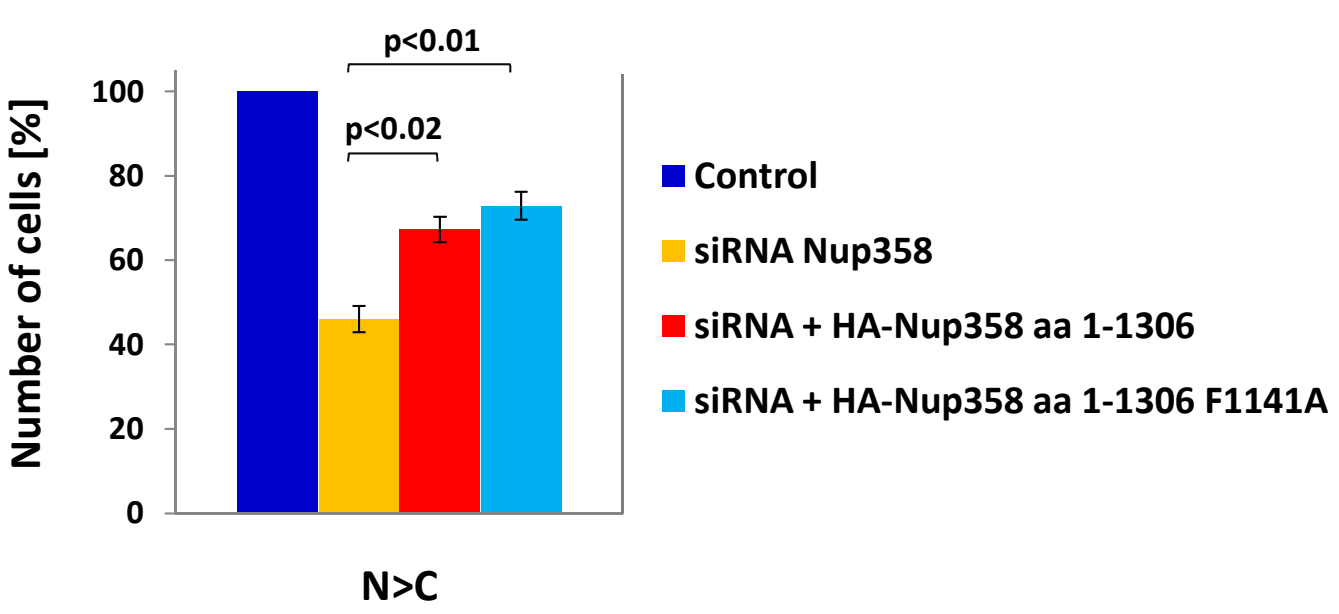

B

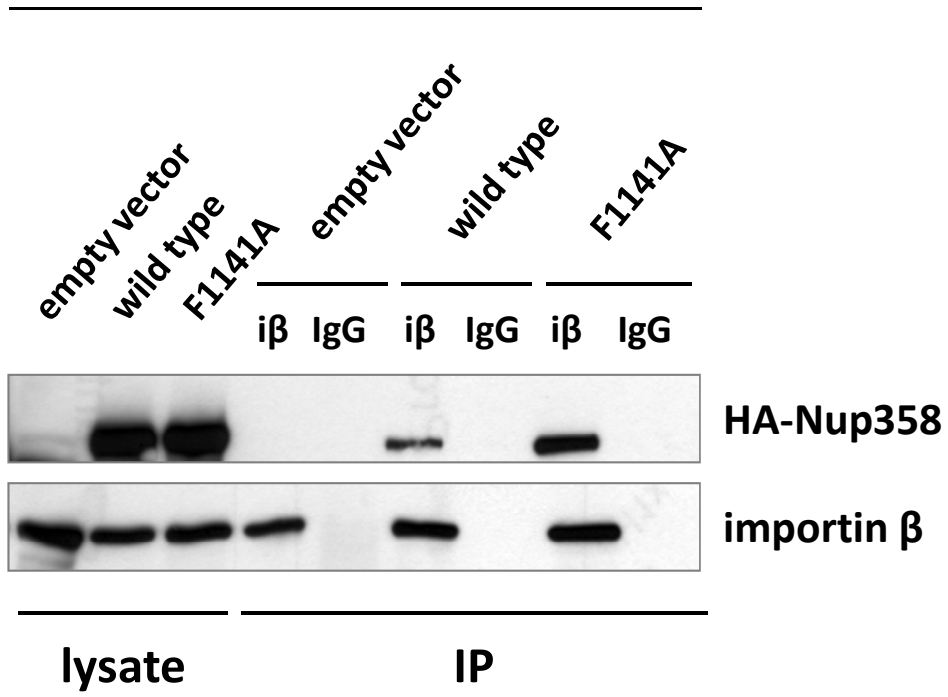

Figure 3-20: The FG-repeat at aa 1141 alone does not mediate nuclear import of DBC-1.

A: Control or Nup358 siRNA-treated HeLa P4 cells were cotransfected with plasmids coding for GFP-DBC-1 and an empty vector, HA-Nup358 aa 1-1306 or HA-Nup358 aa 1-1306 F1141A. $\alpha$-HA- and $\alpha$-Nup358 antibodies were used for the detection of the proteins. For quantification, cellular distribution of GFP-DBC-1 in the absence or presence of the HA-Nup358 truncations was analyzed and grouped into the three categories $\mathrm{N}>\mathrm{C}, \mathrm{N}=\mathrm{C}$ or $\mathrm{C}>\mathrm{N}$ (only $\mathrm{N}>\mathrm{C}$ is shown here). For a better comparison, the number of control cells in the category $\mathrm{N}>\mathrm{C}$ was set to $100 \%$. Error bars indicate the standard deviation from the mean of three independent experiments with $>100$ cells analyzed in each single experiment. The statistical significance of the data was determined using the student's t-test. B: 293T HEK cells were transfected with an empty vector, HA-Nup358 aa 1-1306 or HA-Nup358 aa 1-1306 F1141A, followed by immunoprecipitations using either an $\alpha$-importin $\beta$ antibody (i $\beta$ ) or rabbit IgG as specificity control. Bound proteins were separated by SDS-PAGE and analyzed by western blotting using $\alpha$-HA- or $\alpha$-importin $\beta$ antibodies, respectively. The input corresponds to $1 \%$ of total lysate. 
This suggests that localization of the Nup358 region mediating nuclear import of DBC-1 at the outer nuclear membrane is not sufficient for transport. Instead, the incorporation into the nuclear pore is required to compensate for the loss of endogenous Nup358.

\subsubsection{The FG-repeat at amino acid 1141 alone does not mediate nuclear import of DBC-1}

As shown in Figure 3-18 A+B, there was a clear difference in the ability of the $\mathrm{N}$-terminal fragments aa 1-1170 and aa 1-1133 to rescue import of DBC-1. Interestingly, the additional 30 amino acid sequence contained one FG-repeat at position 1141 whereas fragment aa 1-1133 had only two. In order to analyze the impact of this additional FG-repeat on the ability to rescue import of DBC-1, the phenylalanine of the repeat was mutated to alanine in HA-Nup358 aa 1-1306 as this mutation abolishes binding to importins completely (Patel et al., 2008). Thus, the rescue ability for nuclear import of DBC-1 in Nup358-depleted cells was compared between Nup358 aa 1-1306 wild type and FG-mutant. Both fragments could significantly restore nuclear import of DBC-1 to a similar extent: $67 \%$ (wild type) compared to $73 \%$ (FG-mutant) (Figure 3-20 A). Hence, the additional FG-repeat at position 1141 was not responsible for the ability to compensate for endogenous Nup358.

In this context, it was also interesting to investigate the ability of the additional FG-repeat to bind more importin $\beta$. As an antibody against importin $\beta$ could coimmunoprecipitate Nup358 (Kehlenbach et al., 1999), an immunoprecipitation was carried out with an $\alpha$-importin $\beta$ antibody in cells transfected with fragment aa 1-1306 wild type or F1141A. A transfected empty vector served as negative control. Importin $\beta$ was precipitated efficiently, whereas the IgG controls did not reveal any importin $\beta$ signal (Figure 3-20 B). Wild type as FG-mutant could be bound with equal efficiency.

Consequently, the additional FG-repeat did not result in more binding of the import receptor suggesting that another motif mediates nuclear import of DBC-1. 
A

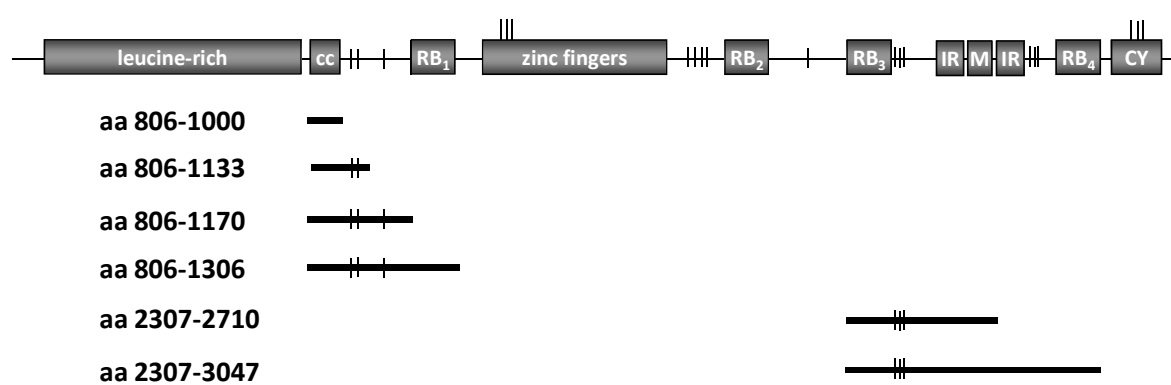

B

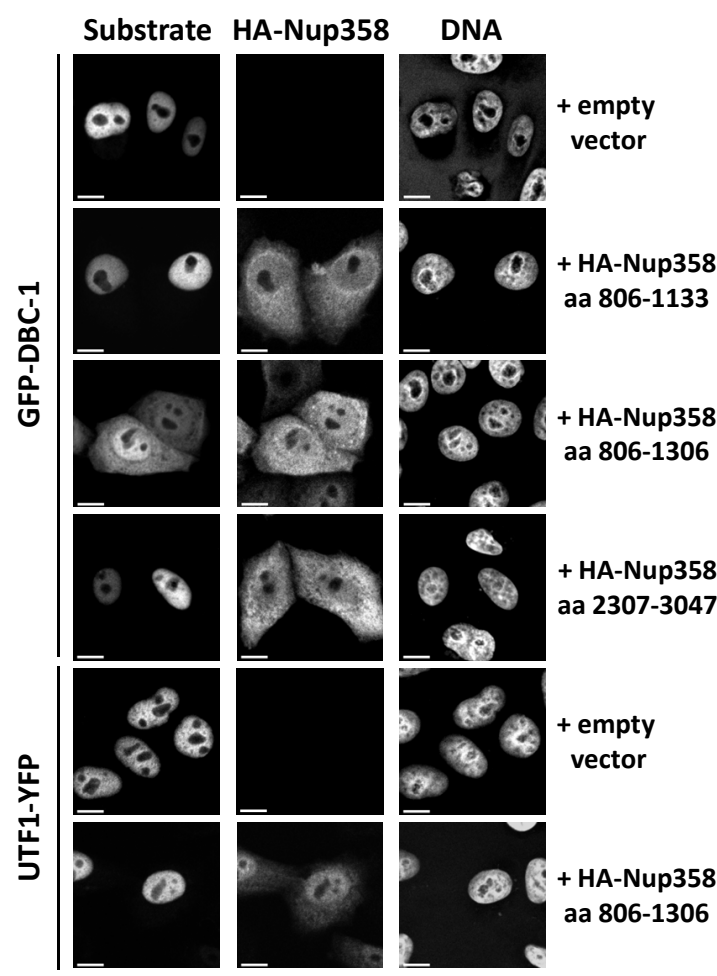

C

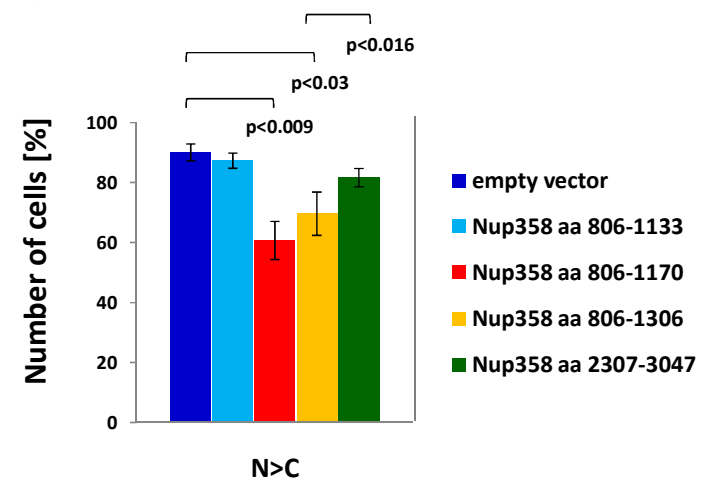

D

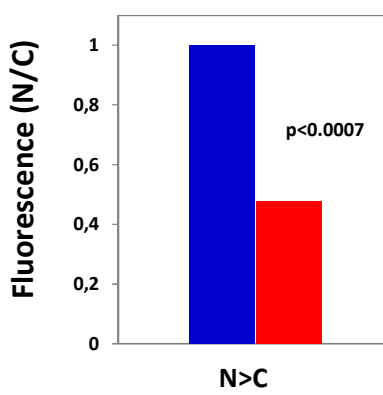

- empty vector

HA-Nup358 aa 806-1306

Figure 3-21: Soluble N-terminal fragments of Nup358 can inhibit nuclear import of DBC-1.

A: Schematic description of HA-tagged Nup358 fragments. They do not contain the sequence for incorporation into the nuclear pore complex. Dashes indicate FG-repeats. B: HeLa P4 cells were cotransfected with plasmids coding for GFP-DBC-1 or UTF1-YFP and an empty vector, HA-Nup358 aa 806-1306 and HA-Nup358 aa 2307-3047, as indicated. The fragments were detected using an $\alpha-H A$-antibody, and DNA was stained by Hoechst. Localization of the proteins was determined by fluorescence microscopy. C: For quantification, cells were grouped into $\mathrm{N}>\mathrm{C}, \mathrm{N}=\mathrm{C}$ and $\mathrm{C}>\mathrm{N}$, and subcellular distribution of GFP-DBC-1 in absence or presence of the indicated HA-Nup358 fragments was analyzed (only $\mathrm{N}>\mathrm{C}$ is shown). $>100$ cells in three independent experiments were counted. Standard deviations indicate variations from the mean of three independent experiments. D: Ratio of nuclear to cytoplasmic fluorescence of cells with GFP-DBC-1 in the nucleus $(N>C)$ in absence or presence of HA-Nup358 aa 806-1306 was calculated. The mean ratio of 32 cells (empty vector) or 46 cells (HA-Nup358 aa 806-1306) is illustrated. The experiment was performed twice with similar outcomes. The statistical significance of the data was determined using the student's t-test (C, D). 


\subsubsection{Analysis of the interaction between Nup358 and DBC-1}

\subsubsection{Soluble fragments of Nup358 inhibit nuclear import of DBC-1}

Assuming that transport complexes containing DBC-1 might interact with a certain region of the N-terminal part of Nup358, nuclear import of DBC-1 might be inhibited by binding to isolated, soluble domains of the region of interest (Figure 3-21 A). Thus, soluble fragments of Nup358 were coexpressed with GFP-DBC-1 in HeLa P4 cells and the localization of DBC-1 was analyzed in absence and presence of the Nup358 fragments. As the short fragments did not contain the complete region required for incorporation into the nuclear pore (own observation), they localized homogenously throughout the entire cellular volume (Figure 3-21 $\mathrm{B}$ ). GFP-DBC-1 localized to the nucleus in $90 \%$ (Figure 3-21 $\mathrm{B}+\mathrm{C}$ ). Presence of the fragment aa $806-1170$ that included the coiled-coil domain and the three FG-repeats, resulted in a significant inhibition of nuclear import of DBC-1 with only $60 \%$ of cells having the protein in the nucleus (Figure 3-21 C). A similar inhibitory effect was achieved by cotransfection with Nup358 aa 806-1306 that extends the Nup358 fragment beyond the RanBD1 whereas Nup358 aa 806-1133 did not inhibit at all (87 \%) (Figure 3-21 B+C).

In order to detect slight changes in the efficiency of nuclear import of DBC-1 in cells which were still analyzed as $\mathrm{N}>\mathrm{C}$, the ratio of nuclear to cytoplasmic fluorescence of GFP-DBC-1 was measured in absence or presence of HA-Nup358 aa 806-1306. The ratio of nuclear to cytoplasmic fluorescence without the Nup358 fragment was set to 1 which decreased significantly to 0.5 in presence of HA-Nup358 aa 806-1306 (Figure 3-21 D).

To analyze if this effect was specific for the region spanning the coiled-coil region and RanBD1, inhibitory effects of other Nup358 fragments on the localization of DBC-1 were analyzed as control. Nup358 aa 2307-3047, expressing RanBD3 and RanBD4 of Nup358, did not inhibit import (Figure 3-21 $\mathrm{B}+\mathrm{C}$ ). Additionally, the inhibitory effect of Nup358 aa 806-1306 was tested on UTF1-YFP, a protein showing Nup358-independent nuclear import. The protein localized to the nucleus in absence and presence of the soluble Nup358 fragment. 
A

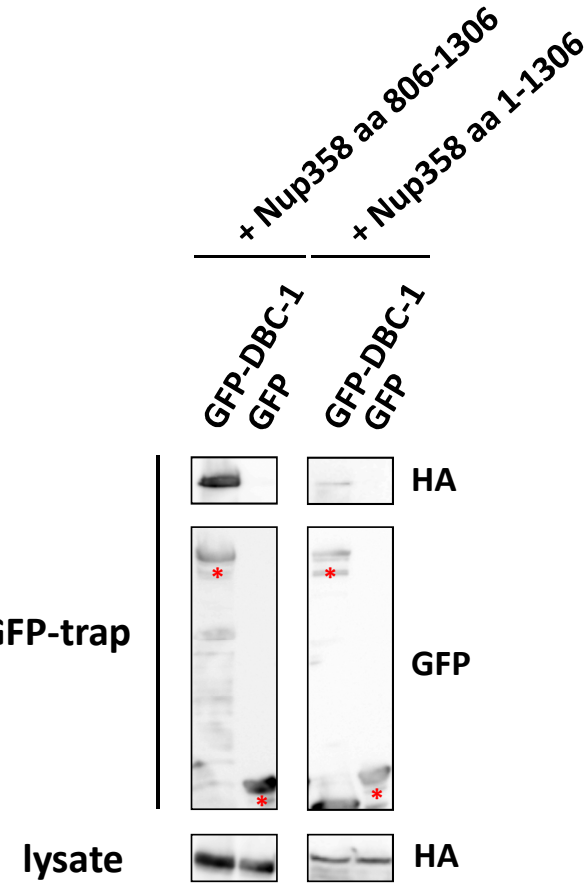

B

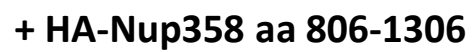

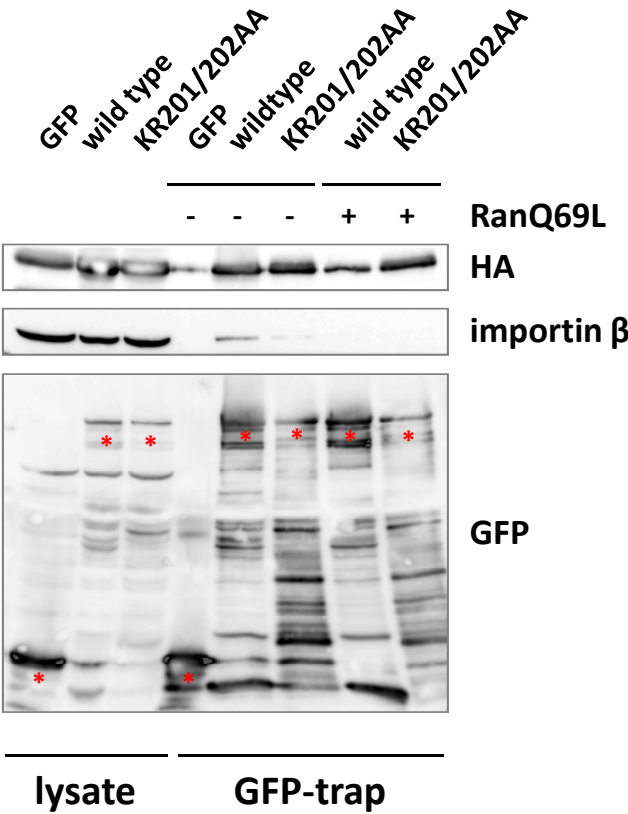

C

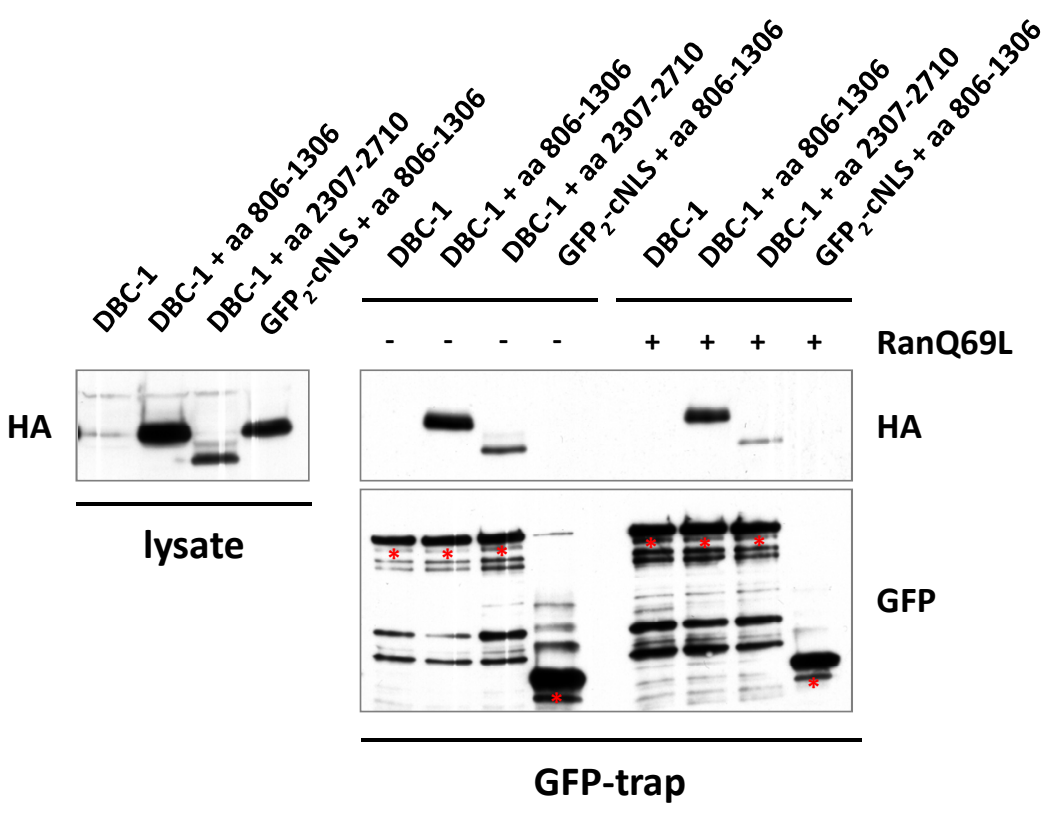


Possibly, the fragments could titrate out importin $\beta$ or the Ran-protein. So the localization of the respective endogenous proteins was determined by immunofluorescence, but the subcellular distribution of neither importin $\beta$ nor Ran changed in presence of the Nup358 fragments (data not shown).

Our results so far point to a specific interaction between the $\mathrm{N}$-terminus of Nup358 and DBC-1 in the region roughly spanning aa 1000-1170. The fragment could not inhibit nuclear import of other proteins; further other regions of Nup358 did not affect localization of DBC-1.

\subsubsection{DBC-1 can interact with the N-terminal part of Nup358}

The findings so far suggested that DBC- 1 interacted with a region within the first third of Nup358. To confirm and further analyze the possible interaction, coimmunoprecipitations were carried out using the GFP-nanotrap to enrich the possible complex between GFP-DBC-1 and the region of interest of Nup358. Thus, 293T HEK cells were cotransfected with GFP-DBC-1 and the soluble fragment HA-Nup358 aa 806-1306 or the fragment containing the anchor sequence, HA-Nup358 aa 1-1306. Cells expressing GFP alone served as negative control. Interaction between the two proteins was detected with an $\alpha-\mathrm{HA}$-antibody in western blot analysis. DBC-1 interacted with the short soluble fragment aa 806-1306 as well as with the fragment containing the anchor domain (Figure 3-22 A) demonstrating an interaction between DBC-1 and the N-terminal region of Nup358 in vivo.

Figure 3-22: DBC-1 can interact with the N-terminal third of Nup358 independently of the NLS and Ran. After transient transfection of 293T HEK cells, immunoprecipitations were performed using the GFP-nanotrap. Bound proteins were separated by SDS-PAGE and analyzed in western blot with either $\alpha-H A-, \alpha$-GFP- or $\alpha$-importin $\beta$ antibodies, respectively. The input corresponds to $1 \%$ of total lysate.

A: Plasmids coding for GFP-DBC-1 or GFP were cotransfected with HA-Nup358 aa 806-1306 or HA-Nup358 aa 1-1306. B: Plasmids coding for GFP-DBC-1, GFP-DBC-1 KR201/202AA or GFP were cotransfected with HA-Nup358 aa 806-1306. Immunoprecipitation was performed in absence (-) or presence (+) of RanQ69L, which had been loaded with GTP. C: GFP-DBC-1 was cotransfected with either an empty vector,

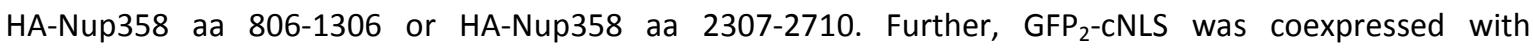
HA-Nup358 aa 806-1306. Bands above the red asterisk indicate immunoprecipitated GFP-proteins. 
As DBC-1 could not be expressed as recombinant protein in bacteria, binding assays with recombinant proteins could not be performed to test if this interaction was direct or mediated by bridging proteins. To exclude transport factors mediating interaction between the FG-repeats of Nup358 and DBC-1, immunoprecipitations were performed in the presence or absence of recombinant RanQ69L. Further, binding of the NLS-mutant (KR201/202AA) of DBC-1 to the soluble Nup358 fragment was analyzed as importin $\alpha$ could not function as bridging protein here. 293T HEK cells were cotransfected with either DBC-1 full-length or the NLS-mutant with Nup358 aa 806-1306. Cells expressing GFP alone served as negative control. After immunoprecipitation of the GFP-proteins using the GFP-nanotrap, an interaction between DBC-1 wild type or the NLS-mutant with Nup358 aa 806-1306 was detectable in the absence of RanQ69L (Figure 3-22 B). Importin $\beta$ could be detected in the complex with DBC-1 wild type whereas the amount in the complex between Nup358 and the NLS-mutant decreased. Transportin was almost undetectable in the complex (data not shown). Remarkably, presence of RanQ69L abolished interaction of importin $\beta$, but HA-Nup358 aa 806-1306 could still bind to DBC-1 wild type and the NLS-mutant, suggesting an NTR-independent interaction. GFP alone showed slight interaction with the Nup358 fragment; but in comparison with the amount of protein bound to the GFP-DBC-1 the signal was rather weak.

As a control, binding of HA-Nup358 aa 806-1306 to another NLS-containing protein, $\mathrm{GFP}_{2}-\mathrm{CNLS}$, was tested in absence or presence of RanQ69L in immunoprecipitations as carried out before. The fragment, however, interacted with GFP-DBC-1 but not with $\mathrm{GFP}_{2}$-CNLS (Figure 3-22 C). Presence of RanQ69L-GTP weakened the interaction to DBC-1 as less Nup358 could be precipitated with an increased amount of bound DBC-1 suggesting besides the Ran-independent interaction between DBC-1 and Nup358 also an association driven by Ran. Additionally, binding of GFP-DBC-1 to another region of Nup358, aa 2307-2710 (Figure 3-21 A) was analyzed. Compared to the strong interaction with Nup358 aa 806-1306, binding of DBC-1 to the fragment aa 2307-2710 was weak and further reduced in presence of RanQ69L (Figure 3-22 C). However, a specific interaction cannot be ruled out with this region of Nup358, but independent of nuclear transport as a similar fragment aa 2307-3047 had no effect on nuclear import of DBC-1 in the cotransfection experiments. 
In summary, these experiments clearly disclose a specific binding of Nup358 aa 806-1306 to DBC-1 driven by two mechanisms. One is independent of Ran and NLS, the other is mediated in a Ran-sensitive way.

\subsubsection{Nup358 can rescue the import defect of other reporter proteins}

DBC-1 is transported via the importin $\alpha / \beta$ pathway (see Figures $3-9 \mathrm{C}$ and $3-10$ ). So the rescue ability of the Nup358 truncations was extended to well-defined import cargos like NES-GFP ${ }_{2}$-CNLS (importin $\alpha / \beta$-dependent) (Hutten et al., 2008) and $\mathrm{GR}_{2}-\mathrm{GFP}_{2}-\mathrm{M} 9$ (transportin-dependent) (Hutten et al., 2009) to see whether the same region in Nup358 was required for promoting different nuclear import pathways.

\subsubsection{The N-terminal part of Nup358 mediates nuclear uptake of NES-GFP ${ }_{2}$-CNLS}

As shown before, the importin $\alpha / \beta$ cargo NES-GFP ${ }_{2}$-cNLS stayed mostly cytoplasmic upon loss of Nup358 (Figure 3-23 A). Importantly, it accumulated in the nucleus in the presence of mRFP-Nup358. The number of cells displaying nuclear localization of the protein increased from $44 \%$ in knockdown cells to $95 \%$ in cells expressing mRFP-Nup358 (Figure 3-23 B).

Moreover, the N-terminal Nup358 truncations were analyzed for their rescue ability. As demonstrated for DBC-1, fragment aa 1-1133 failed to rescue nuclear import of NES-GFP 2 -CNLS in cells depleted of Nup358. Strikingly, only additional 30 amino acids in fragment HA-Nup358 aa 1-1170 were able to mediate nuclear import of the reporter protein to $85 \%$ of control level. The fragment aa 1-1306 including RanBD1 resembled almost localization like in control cells (90\%) (Figure 3-23 A+B) demonstrating that nuclear import of the NLS-cargos can occur without pore-associated Ran-binding domains and RanGAP1.

As demonstrated for DBC-1, the first third of Nup358 sufficed to compensate for endogenous Nup358, but incorporation into the nuclear pore was required. To test if localization at the outer nuclear membrane sufficed to compensate for the loss of 


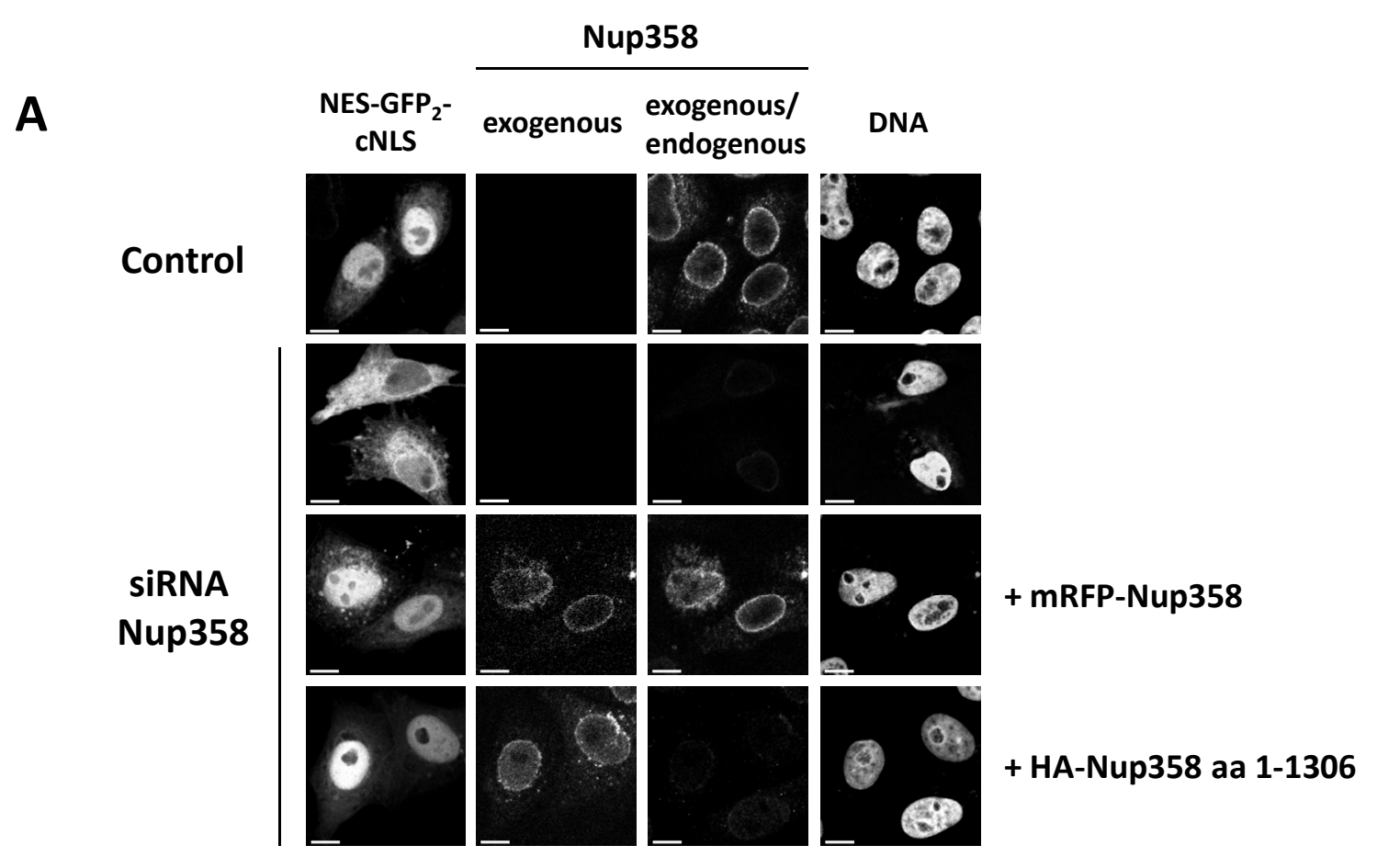

B

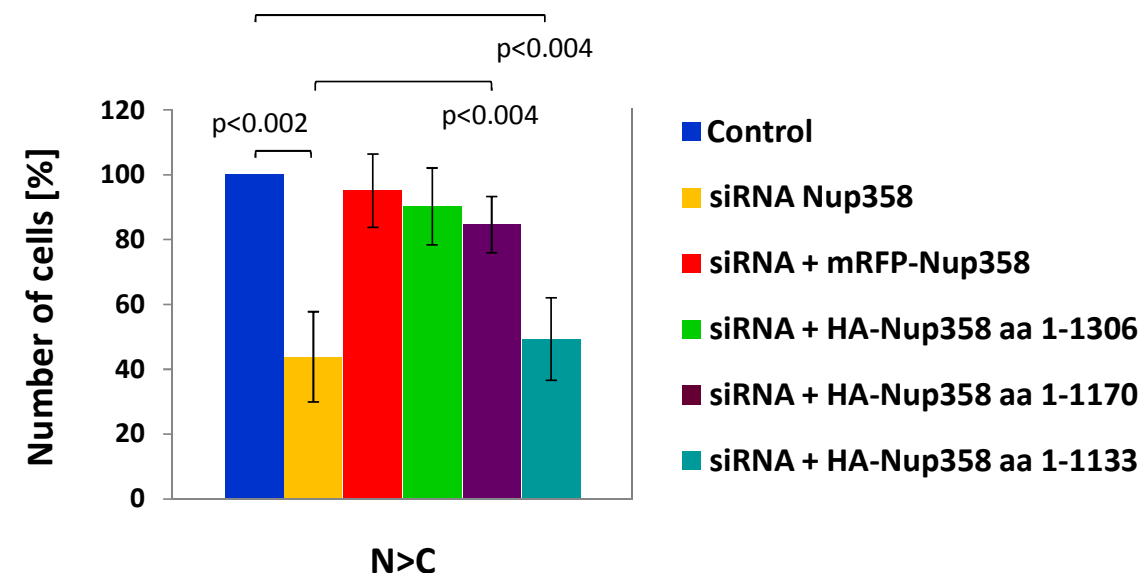


Nup358 in case of NES-GFP 2 -cNLS, the rescue ability of the fusion with the KASH-domain of Syne 1B was analyzed in Nup358-depleted cells (see Figure 3-19). Neither HA-Syne 1B nor the fusion HA-Syne 1B-Nup358 aa 806-1306 could rescue the import of the reporter protein (data not shown) similar to the experiment using DBC-1 as a reporter protein. This means that incorporation into the nuclear pore was required to compensate for endogenous Nup358.

In summary, the $\mathrm{N}$-terminal fragment aa 1-1170 was sufficient to mediate nuclear import of cargos using the importin $\alpha / \beta$ pathway. The Nup358/RanGAP1 complex and the RanBDs of Nup358 were not needed. Further, incorporation into the nuclear pore was required for import of both substrates. This argues for an importin $\alpha / \beta$ pathway-specific interaction between substrate and Nup358.

Figure 3-23: The N-terminus of Nup358 is sufficient to mediate nuclear import of NES-GFP ${ }_{2}$-cNLS.

A: Control or Nup358 siRNA-treated HeLa P4 cells were cotransfected with plasmids coding for NES-GFP ${ }_{2}$-CNLS and a siRNA-resistant mutant of mRFP-Nup358 or HA-Nup358 aa 1-1306. For analysis, endogenous Nup358 and transfected mRFP-Nup358 was detected with an $\alpha$-Nup358 antibody; the Nup358 fragment was recognized by an $\alpha$-HA-antibody. DNA was visualized by Hoechst.

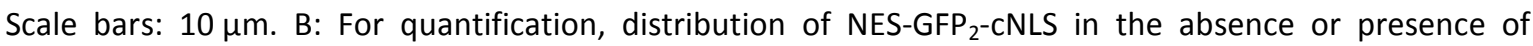
mRFP-Nup358, HA-Nup358 aa 1-1306, aa 1-1170 or aa 1-1133 was analyzed and grouped into the three categories $\mathrm{N}>\mathrm{C}, \mathrm{N}=\mathrm{C}$ or $\mathrm{C}>\mathrm{N}$ (only $\mathrm{N}>\mathrm{C}$ is shown here). As the $\alpha$-Nup358 antibody also detected mRFP-Nup358 thereby masking an efficient depletion, only cells were characterized whose bystanders clearly lacked Nup358, suggesting an efficiently transfected area of cells. Due to great experiment to experiment variations in the localization between nucleus and cytoplasm in control cells, the localization in knockdown cells with or without overexpression of the Nup358 truncations was calculated in relation to the distribution in control cells. For this, the number of cells in the category $\mathrm{N}>\mathrm{C}$ was normalized to $100 \%$. Error bars indicate the standard deviation from the mean of three independent experiments with $>100$ cells analyzed in each single experiment. The statistical significance of the data was determined using the student's t-test. 


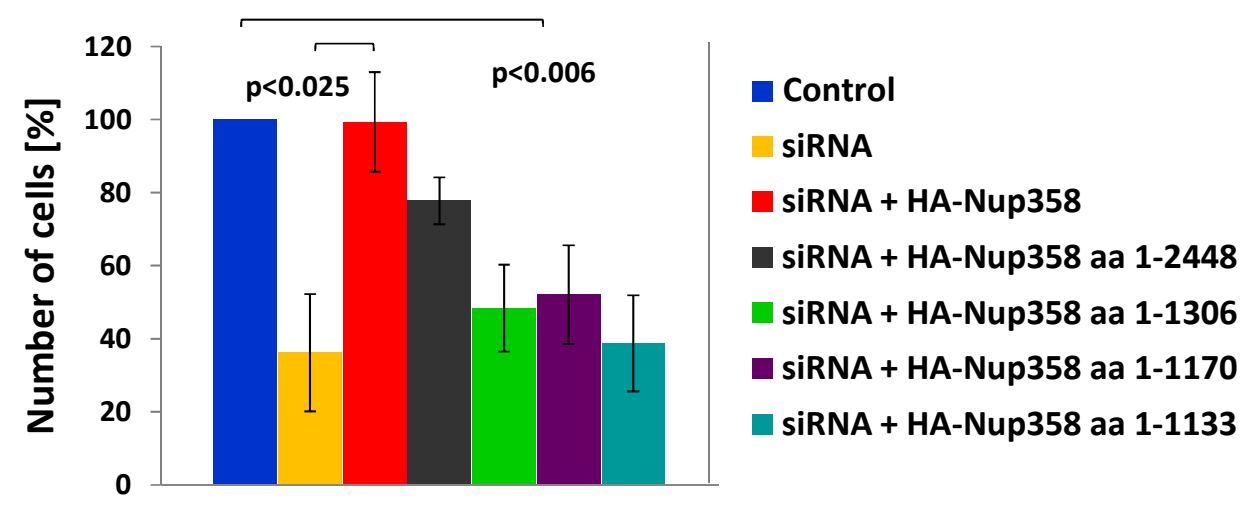

\section{$\mathrm{N}>\mathrm{C}$}

Figure 3-24: Additional regions of Nup358 are required for nuclear import of a transportin cargo.

Control or Nup358-depleted HeLa P4 cells were cotransfected with plasmids coding for $\mathrm{GR}_{2}-\mathrm{GFP}_{2}-\mathrm{M} 9$ (GG-M9) and siRNA-resistant mutants of HA-Nup358 full-length, aa 1-2448, aa 1-1306, aa 1-1170 or aa 1-1133, as indicated.. Addition of $500 \mu \mathrm{m}$ dexamethasone induced nuclear import of the reporter protein which was stopped after 20 min by fixing the cells. HA-Nup358 full-length and fragments were detected with an $\alpha$-HA-antibody, endogenous and exogenous Nup358 full-length were detected with an $\alpha$-Nup358 antibody. For quantification, distribution of GG-M9 in the absence or presence of Nup358 fragments was analyzed and grouped into the three categories $\mathrm{N}>\mathrm{C}, \mathrm{N}=\mathrm{C}$ or $\mathrm{C}>\mathrm{N}$ (only $\mathrm{N}>\mathrm{C}$ is shown here). As the $\alpha$-Nup358 antibody also detected HA-Nup358 full-length thereby masking an efficient depletion, only cells were characterized whose bystanders clearly lacked Nup358, suggesting an efficiently transfected area of cells. Error bars indicate the standard deviation from the mean of three independent experiments with $>100$ cells analyzed in each single experiment. Due to great experiment to experiment variations in the localization between nucleus and cytoplasm in control cells, the localization in knockdown cells with or without overexpression of the Nup358 truncations was calculated in relation to the distribution in control cells. For this, the number of cells in the category $\mathrm{N}>\mathrm{C}$ was normalized to $100 \%$. The statistical significance of the data was determined using the student's t-test. 


\subsubsection{Additional regions of Nup358 are required for nuclear import of a transportin cargo}

Nup358 has been shown to be required for the import of both, importin $\alpha / \beta$ - and transportin-mediated import. Therefore, we also investigated whether the role of the $\mathrm{N}$-terminal region of Nup358 is specific for the importin $\alpha / \beta$ pathway or whether it is also involved in the import of transportin-dependent cargos. Thus, substrates of the transportin-pathway were included into the rescue experiments. Here, we made use of the dexamethasone-inducible reporter $\mathrm{GR}_{2}-\mathrm{GFP}_{2}-\mathrm{M9}$ (GG-M9) as import of this substrate strongly depends on Nup358 (Hutten et al., 2009). The reporter protein was analyzed after cotransfection with different Nup358 truncations in cells depleted of the nucleoporin.

Nuclear accumulation was significantly reduced to $36 \%$ after siRNA treatment (Figure 3-24). Presence of full-length HA-Nup358 could compensate for this import defect. As expected, the anchor fragment aa 1-1133 did not influence the localization of GG-M9, and the reporter still accumulated in the cytoplasm. In contrast to the analyzed cargos of the importin $\alpha / \beta$ pathway, the $N$-terminal fragment aa 1-1170 and aa 1-1306 were not able to compensate for the loss of Nup358 with only $50 \%$ of cells showing nuclear distribution (Figure 3-24). Only the fragment aa 1-2448, which expresses three RanBDs could partially rescue nuclear import of GG-M9 (77\%).

After analyzing the required domains of Nup358 for nuclear import of the reporter protein GG-M9, we next investigated the transportin-dependent cargo HIV-1 Rev to see if the different necessities of import-mediating regions are specific for distinct import pathways. 


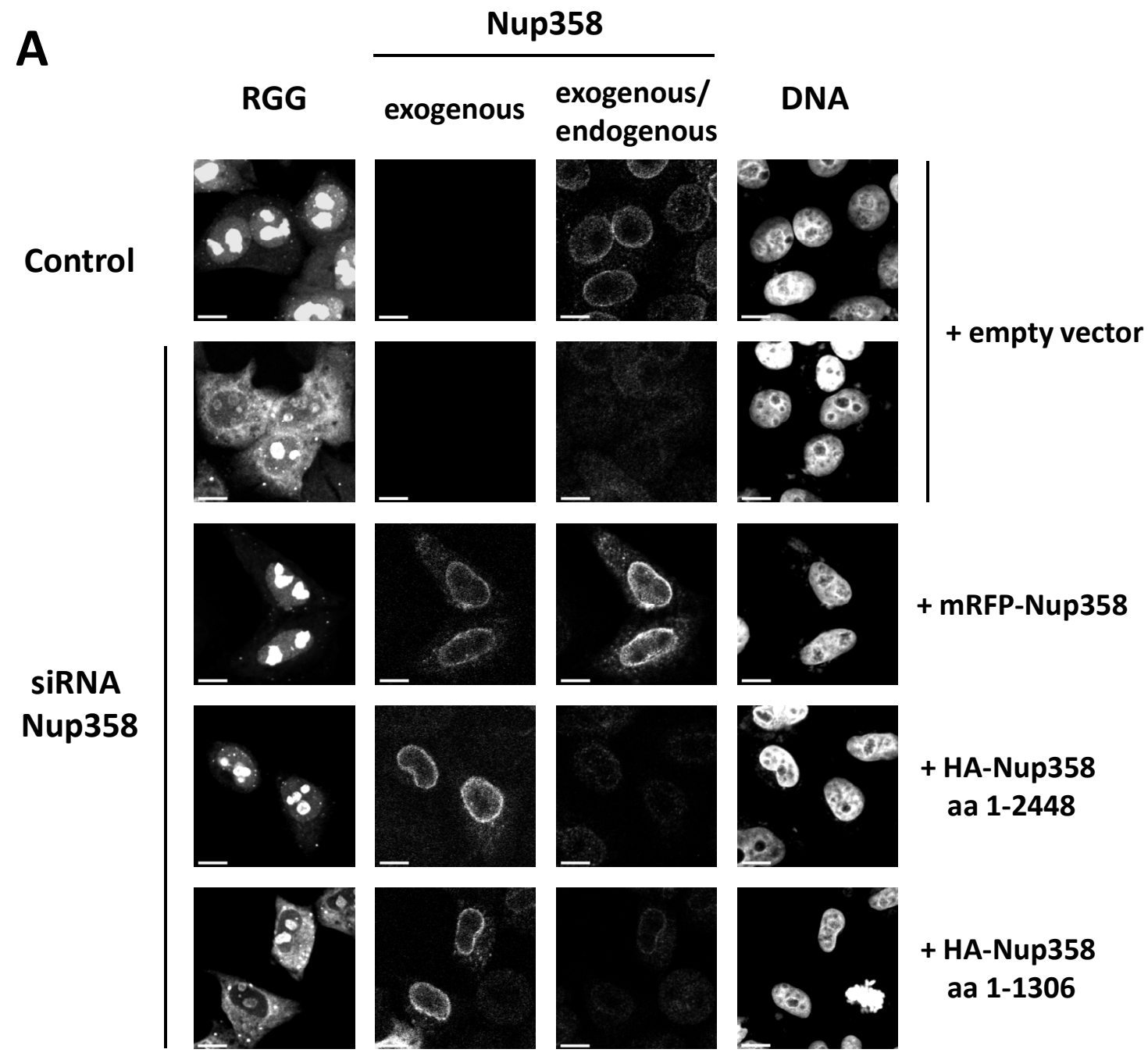

B

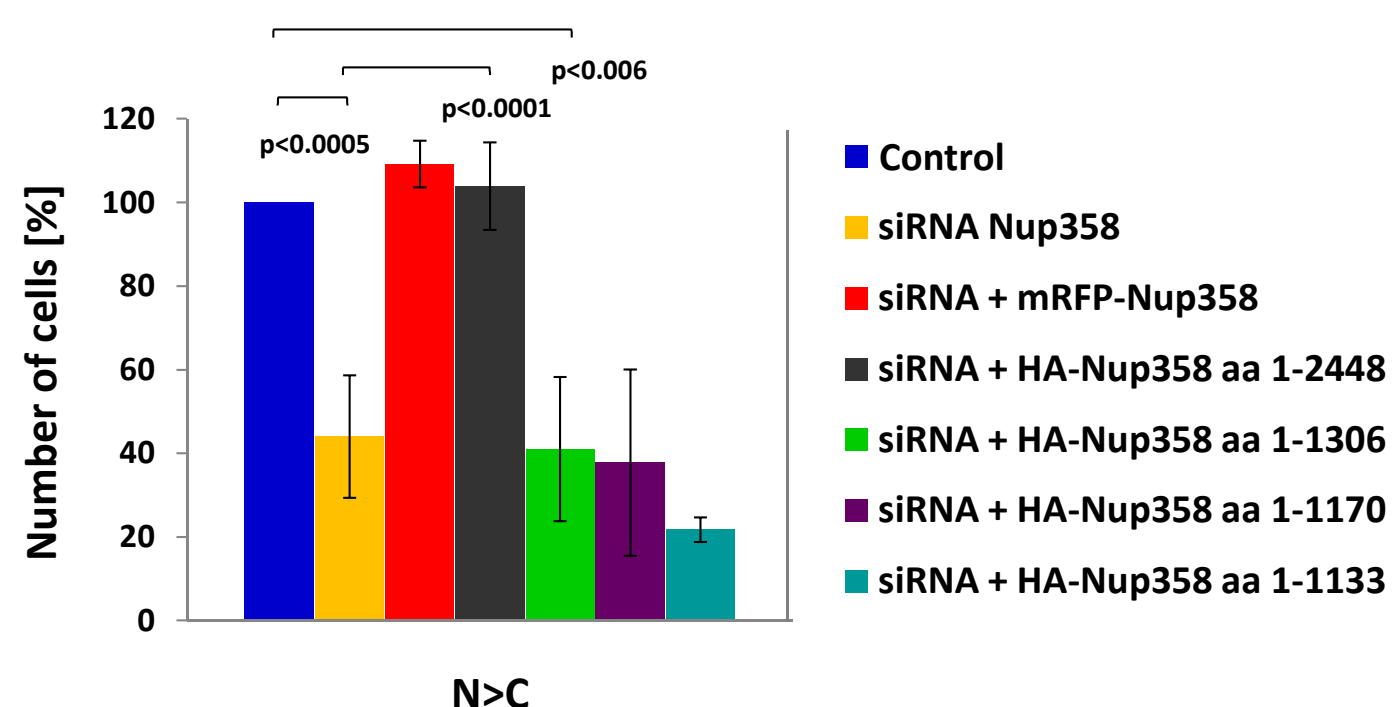




\subsubsection{A similar region of Nup358 as needed for the transportin reporter can mediate nuclear import of the HIV-1 Rev protein}

As already reported nuclear localization of HIV-1 Rev depends on Nup358 and transportin (Hutten et al., 2009). The dexamethasone-inducible reporter protein Rev-GR-GFP (RGG) (Love et al., 1998) was cotransfected with certain Nup358 truncations in Nup358-depleted cells. The decrease from $100 \%$ to $44 \%$ in cells lacking Nup358 was significant (Figure 3-25 A+B). Remarkably, presence of mRFP-Nup358 fully recovered nuclear accumulation of HIV-1 Rev. As observed for GG-M9, DBC-1 and NES-GFP ${ }_{2}$-CNLS, the N-terminal anchor fragment aa 1-1133 did not rescue nuclear import of HIV-1 Rev.

Similarly, the truncations aa 1-1306 and 1-1170 could not compensate for endogenous Nup358 as the number of cells with HIV-1 Rev in the nucleus did not increase significantly (Figure 3-25 A+B). In the presence of Nup358 fragment aa 1-2448, however, the number of cells having HIV-1 Rev in the nucleus reached control level.

In summary, in contrast to DBC-1 and the NLS-cargo using the importin $\alpha / \beta$ pathway, nuclear import of Nup358-dependent substrates transported by transportin could not be mediated by the N-terminal part of Nup358. However, a fragment containing three RanBDs, aa 1-2448, could rescue these substrates. As for DBC-1 and NES-GFP - -CNLS, Nup358-associated RanGAP1 was not required for nuclear import.

Figure 3-25: A similar region of Nup358 as needed for the transportin reporter protein can mediate nuclear import of the HIV-1 Rev protein.

A: Control or Nup358 siRNA-treated cells were cotransfected with plasmids coding for the HIV-1 Rev reporter Rev-GR-GFP (RGG) and an empty vector or siRNA-resistant mutants of mRFP-Nup358, HA-Nup358 aa 1-2448, aa 1-1306, aa 1-1170 or aa 1-1133, respectively. Addition of dexamethasone induced nuclear import of the reporter protein, and cells were fixed after $20 \mathrm{~min}$. HA-Nup358 fragments were detected with an $\alpha$-HA-antibody; endogenous Nup358 and mRFP-Nup358 was detected with an $\alpha$-Nup358 antibody. DNA was stained by Hoechst. Scale bar: $10 \mu \mathrm{m}$. B: For quantification, the localization of RGG in absence or presence of the Nup358 fragments was analyzed, and grouped into the three categories $\mathrm{N}>\mathrm{C}$, $\mathrm{N}=\mathrm{C}$ or $\mathrm{C}>\mathrm{N}$ (only $\mathrm{N}>\mathrm{C}$ is shown here). As the $\alpha$-Nup358 antibody also detected mRFP-Nup358 thereby masking an efficient depletion, only cells were analyzed whose bystanders clearly lacked Nup358, suggesting an efficiently transfected area of cells. Error bars indicate the standard deviation from the mean of three independent experiments with > 100 cells analyzed in each single experiment. Due to great experiment to experiment variations in the localization between nucleus and cytoplasm in control cells, the localization in knockdown cells with or without overexpression of the Nup358 truncations was calculated in relation to the distribution in control cells. For this, the number of cells in the category $\mathrm{N}>\mathrm{C}$ of control cells was normalized to $100 \%$. The statistical significance of the data was determined using the student's t-test. 


\subsubsection{Different nuclear transport pathways require distinct domains of Nup358 for} their import

For a better comparison between the rescue ability of certain truncations of Nup358 towards DBC-1, NES-GFP 2 -CNLS, GG-M9 and HIV-1 Rev, all corresponding quantifications were summarized in one graph. Hence, cells with a predominantly nuclear localization of the substrate decreased to $50 \%$ upon Nup358 depletion (Figure 3-26). Exogenous Nup358 could compensate for the loss of endogenous Nup358, but the efficiency of rescue differed among the substrates. Nuclear import of all substrates occurred in the absence of the Nup358/RanGAP1 complex as the fragment aa 1-2448 lacking the RanGAP1 interaction site was able to mediate import.

Strikingly, the regions required for nuclear import varied dramatically between the distinct pathways. The $\mathrm{N}$-terminal fragment aa 1-1306 failed to mediate nuclear import of cargos using the transportin pathway whereas it could promote transport of DBC-1 and NES-GFP 2 -CNLS, both using the importin $\alpha / \beta$ pathway. Noticeably, the anchor fragment aa 1-1133 failed to compensate for the loss of endogenous Nup358 at all.

For future rescue experiments, a certain truncation will always be analyzed with full-length Nup358 in parallel. The abundance of this truncation to mediate import is set in direct relation to full-length Nup358. Hence, comparison among different substrates will be much easier. For example, Nup358 aa 1-2448 can mediate nuclear uptake of DBC-1 and GG-M9 to a similar extent (77 \% rescue efficiency) whereas HIV-1 Rev can be imported much more efficiently which could result from a more efficient rescue with full-length Nup358.

In summary, it was clearly demonstrated that the region within Nup358 required for efficient nuclear import varies considerably between the importin $\alpha / \beta$ and transportin pathway. For all substrates analyzed here, pore-associated RanGAP1 was not required for nuclear import, suggesting that the soluble protein is sufficient to fulfill its function. 


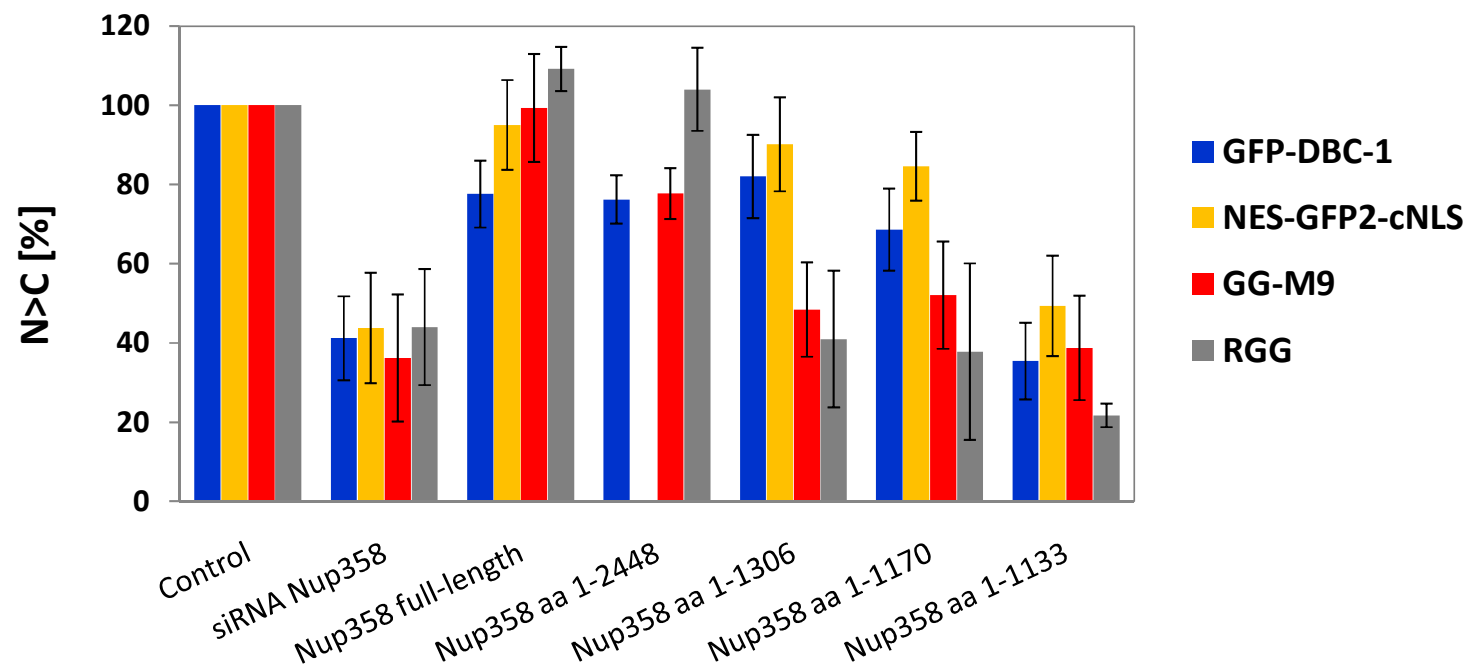

Figure 3-26: Different nuclear transport pathways require distinct domains of Nup358 for their import.

For a better comparison, the quantification of the rescue ability of certain Nup358 fragments towards GFP-DBC-1, NES-GFP - -CNLS, GR - GFP $_{2}-\mathrm{M} 9$ (GG-M9) and the HIV-1 Rev-reporter RGG were summarized in one graph. As before, error bars indicate the standard deviation from the mean of three independent experiments with $>100$ cells analyzed in each single experiment. Note that Nup358 aa 1-1306 rescues DBC-1 and NES-GFP 2 -cNLS, but not the transportin cargos $\mathrm{GR}_{2}-\mathrm{GFP}_{2}-\mathrm{M} 9$ and HIV-1 Rev. 



\section{DISCUSSION}

\subsection{Nuclear accumulation of a subset of proteins depends on Nup358}

Nup358 is a multi-domain protein with several distinct domains involved in diverse cellular mechanisms. Some of its characteristic features like FG-repeats that interact with NTRs, four Ran-binding domains (RanBDs), the associated RanGAP1 and its filamentous localization at the cytoplasmic face of the NPC may be directly linked to nuclear transport. Hence, Nup358 might function as an assembly/disassembly platform for the transport complexes before or after translocation through the NPC (Yokoyama et al., 1995). Indeed, NLS-cargos that are recognized by NTRs were shown to initially dock at the cytoplasmic filaments before translocation through the nuclear pore (Richardson et al., 1988; Pante et al., 1993).

On the other hand, Nup358 was reported to be dispensable for nuclear uptake of NLS- and M9-cargos (Walther et al., 2002), the glucocorticoid receptor (Salina et al., 2003) and the transcription factor NFAT (Hutten et al., 2006). By contrast, nuclear uptake of some proteins in Drosophila cells (Forler et al., 2004; Sabri et al., 2007), and artificial reporters using the importin $\alpha / \beta$ or transportin pathway were significantly slowed down in cells depleted of Nup358 via RNA interference (Hutten et al., 2008; Hutten et al., 2009). Besides these artificial reporter proteins with well-defined NESs and NLSs, the HIV-1 Rev protein and other cellular proteins like DDX43, DDX59, Sox 6 and DBC-1 were identified as Nup358-dependent (Hutten et al., 2009) (Hutten, 2007). They accumulate in the cytoplasm after depletion of Nup358 or appear as extranuclear aggregates like Sox 6.

As only a small subset of proteins show Nup358-dependent nuclear transport, the role of this nucleoporin in nuclear uptake of proteins seems to be very specific. Hence, the function of Nup358 in the nuclear accumulation of proteins has been analyzed in the course of this work. We hoped to gain insight into the import pathways of a number of interesting proteins and into the role of a prominent nucleoporin in transport. 


\subsubsection{Nup358 depletion affects nuclear import and not export of the reporter proteins}

Theoretically, the cytoplasmic accumulation of the different reporter proteins in Nup358-depleted cells might result either from impaired import or accelerated export. However, HIV-1 Rev localizes predominantly to the cytoplasm in Nup358-depleted cells under conditions that specifically block CRM1 activity, suggesting a role of Nup358 in nuclear import rather than export (Hutten et al., 2009). The dexamethasone-inducible nuclear import system (Love et al., 1998) provides an excellent method to analyze import kinetics and to distinguish whether import and not export are affected by the depletion of Nup358 in living cells. The decreased kinetic of nuclear uptake of the transportin cargo $\mathrm{GR}_{2}-\mathrm{GFP}_{2}-\mathrm{M9}$ (GG-M9) upon Nup358 depletion strongly points to inhibition of nuclear import rather than accelerated export of the reporter protein (Hutten et al., 2009).

For DDX43, DDX59, Sox 6, DBC-1 and the other cellular proteins characterized as Nup358-dependent, it cannot be completely ruled out that export rather than import is affected. However, CRM1 as exportin can be excluded for DDX43, DDX59, Sox 6 and DBC-1 as LMB treatment to inhibit CRM1 activity did not lead the proteins to the nucleus in Nup358-depleted cells as shown for HIV-1 Rev (data not shown) (Hutten et al., 2009). So far, only CRM1-cargos have been analyzed in Nup358-depleted cells because it is the major NTR mediating export of proteins and mRNA. A small decrease of CRM1-mediated export in cells with reduced levels of Nup358 was reported (Bernad et al., 2004). Therefore, a stimulated nuclear export is rather unlikely (Hutten et al., 2006). 


\subsection{Distinct import pathways are affected by depletion of Nup358}

\subsubsection{DDX43 and DDX59 bind to different import receptors}

So far, only cargos using the importin $\alpha / \beta$ or transportin pathway were identified as Nup358-dependent. Import factors for DDX43 and DDX59 have not been elucidated yet, hence it was interesting to identify their possible NTRs in order to analyze Nup358's possible role in additional import pathways. Although both proteins belong to the group of DEAD-box helicases, they show different binding behaviors towards NTRs. DDX43 associates with importin 7, importin 9 and importin 13, whereas DDX59 interacts with importin 7, transportin and importin 5. Hence, importin 7 together with importin 13 might be the import receptors involved in Nup358-dependent transport of proteins. Further detailed studies will be required to analyze if the bound NTRs can also mediate import of the cargo like import assays in permeabilized cells.

A specific interaction with an import receptor in vitro does not necessarily qualify this importin as a functional import mediator in vivo as observed for HIV-1 Rev (Arnold et al., 2006) (Hutten et al., 2009). The responsible NLS has to be identified and characterized. Although depletion of an NTR by RNAi can affect transport of many other nuclear proteins, it will demonstrate the dependence of a cargo to the respective NTR.

Besides importin $\alpha$, also importin 7 can function as an adaptor for importin $\beta$, for example for histone $\mathrm{H} 1$ (Jäkel et al., 1999). As this receptor interacts with both helicases, its role as adaptor has to be further analyzed.

In addition to the identification of potential import receptors, nuclear export of DDX43 and DDX59 has also been investigated during this work because there is a small cytoplasmic pool of proteins which might result from export. However, inhibition of CRM1 with LMB does not affect the subcellular localization of DDX43 and DDX59 (data not shown). Perhaps other exportins besides CRM1 are involved in nuclear export of these proteins. 


\subsubsection{Several NTRs can mediate nuclear uptake of Sox 6}

In contrast to DDX43 and DDX59, the SRY family member Sox 6 still localizes to the nucleus in cells lacking Nup358, but in addition exhibits extranuclear aggregations that cannot be observed in control cells. The nature of these cytoplasmic aggregates and the question whether they are caused by decreased nuclear import in the absence of Nup358 requires further characterization.

In order to find possibly interacting NTRs of Sox 6, binding assays were performed. Importin $\beta$ alone, transportin and exportin 4 can interact very efficiently with Sox 6 . They can also mediate nuclear uptake of Sox 6 in vitro. To search for the import-mediating NTR in vivo, several putative NLSs have been mutated. Although a respective mutant of Sox 6 cannot bind exportin 4, nuclear import is not affected. Similarly, mutations of a putative PY-NLS-motif that could mediate the interaction with transportin or a potential cNLS for binding of importin $\beta$ do not lead to impaired import of a C-terminal fragment of Sox 6 . Even a fragment with both mutations in parallel (PY-NLS and CNLS) accumulates to the nucleus (data not shown).

Altogether, these findings strongly argue that indeed several NTRs can promote nuclear import of Sox 6 in vivo, and hence compensate for each other.

Besides the import mechanism of Sox 6 , its possible nuclear export was investigated as well. Other proteins of this family have a functional NES, and display CRM1-dependent export (Dork et al., 1998; Gasca et al., 2002; Rehberg et al., 2002). However, Sox 6 does not contain a similar NES, and no other NES-like motif has been found using an online NES-prediction program. LMB treatment of Sox 6 full-length and its fragments did not result in altered subcellular localization (data not shown). This suggests that either CRM1 is not the export mediating receptor, and possible other exportins are involved in translocation of Sox 6 to the cytoplasm or Sox 6 is not exported at all.

In summary, besides the importin $\alpha / \beta$ - and the transportin-dependent pathway, several other pathways seem to be affected by depletion of Nup358. 


\subsubsection{The importin $\alpha / \beta$ complex mediates nuclear import of DBC-1}

A major emphasis of this work was the analysis of the nuclear transport mechanism of DBC-1, which has not yet been characterized so far. In binding assays, the interaction between the importin $\alpha / \beta$ complex and DBC-1 was very strong and efficient. Other importins like importin $\beta$, transportin, importin 7 and importin 13 did associate with DBC-1 in vitro as well. A mutation of KR201/202AA within the putative NLS (aa 200-219) (Sundararajan et al., 2005) abolishes binding of importin $\alpha$ whereas importin $\beta$ alone and transportin can still bind to some extent. Apparently, this interaction is not sufficient to mediate import of the mutant which accumulates in the cytoplasm pointing to an importin $\alpha / \beta$-dependent nuclear import of DBC-1 in vivo.

\subsubsection{The coiled-coil region of DBC-1 is involved in the Nup358 dependency}

In addition to clarifying the import pathways of the substrates, it is also interesting to reveal the region within the protein mediating Nup358 dependency. Hence, the localization of different fragments of DBC-1 was analyzed in cells depleted of Nup358. The responsible motif might be located downstream of the NLS as in cells lacking Nup358 the $\mathrm{N}$-terminal fragment aa 1-220 (+NLS) localizes to the nucleus whereas the C-terminal fragment aa 200-923 accumulates in the cytoplasm. Interestingly, the coiled-coil domain of DBC-1 (aa 794-923) might mediate Nup358 dependency. A mutant lacking this domain is nuclear in cells depleted of Nup358 in contrast to full-length DBC-1. Additionally, the dependency can be transferred to a Nup358-indepenent reporter protein, $\mathrm{GFP}_{2}$-CNLS (Hutten et al., 2006). Here, a fusion of the coiled-coil domain of DBC-1 to GFP ${ }_{2}$-CNLS resulted in cytoplasmic accumulation of the reporter in Nup358-depleted cells. This behavior is comparable to the transport characteristics of the reporter protein NES-GFP - -CNLS as presence of an NLS- and NES-sequence leads to continuous import and export of the reporter protein. Hence, each round of shuttling results in an increasing cytoplasmic accumulation of the protein due to slower nuclear import in cells depleted of Nup358 (Hutten et al., 2008; Hutten et al., 2009). This suggests that the coiled-coil domain of DBC-1 might mediate nuclear export, and shuttling of the protein causes the cytoplasmic accumulation in cells lacking Nup358. 
Indeed, it was postulated that the coiled-coil domain of DBC-1 is involved in translocation of the protein through the NPC (Sundararajan et al., 2005). Truncations of DBC-1 containing the C-terminal part, p120 and p66, are generated in the nucleus during TNF- $\alpha$ mediated cell death, and localize to the cytoplasm to sensitize cells to apoptosis. However, the underlying mechanism was not analyzed in this study.

CRM1-dependent export can possibly be excluded, as LMB treatment of cells expressing the cytoplasmic fragments of DBC-1, aa $220-923$ or aa $794-923$, did not result in nuclear localization (data not shown). Here, it would be interesting to discover potential interaction partners of the coiled-coil domain of DBC-1, for example by mass spectrometry. Perhaps other export factors than CRM1 are involved in nuclear export of DBC-1.

\subsection{Full-length Nup358 and its truncations are a very powerful tool to investigate the involvement in nuclear transport}

After analyzing the import mechanism of the Nup358-dependent substrates, the transport defect was further characterized from the Nup's point of view. In particular, the responsible domains of the nucleoporin mediating nuclear import of single substrates were analyzed in detail to gain more insight into the underlying molecular mechanism of the role of Nup358 in nuclear transport.

Here, the rescue experiments with exogenous full-length Nup358 and several truncation mutants in Nup358-depleted cells proofed to be a very powerful tool. An introduced silent mutation in the mRNA impairs degradation by the RNAi machinery. As the necessary sequence for NPC-incorporation of Nup358 lies within the first 1133 amino acids, full-length and all truncations localize to the NPC like endogenous Nup358. Moreover, they are expressed according to their calculated size, and all of them can interact with importin $\beta$. Only full-length and fragment aa 1-2684 contain the RanGAP1 interaction site, and hence can bind RanGAP1 demonstrating the functionality of the fragments. Remarkable, this is the first time that a proper localization and functionality of 
exogenous full-length Nup358 could be shown. A previously published full-length GFP-Nup358 showed a weak nuclear rim staining besides a strong cytoplasmic distribution (Joseph et al., 2008). Functional assays were not reported in this publication. Strikingly, HA- as well as mRFP-tagged full-length Nup358 can rescue the transport defects of the substrates DBC-1, NES-GFP 2 -cNLS, GG-M9 and the Rev-reporter RGG in Nup358-depleted cells. Furthermore, HA-Nup358 can also compensate the loss of endogenous Nup358 for other substrates displaying Nup358-dependent import (Hutten, 2007), among them DDX43 and DDX59 demonstrating a specific role of Nup358 in nuclear transport of these proteins (Fischer, 2009).

These rescue experiments are the ultimate control for an RNAi experiment to demonstrate a specific effect of the depletion of Nup358 and exclude off-target effects.

\subsubsection{Pore-associated RanGAP1 and the E3 ligase activity of Nup358 are not required for nuclear import}

In vertebrate cells, a pool of RanGAP1 is associated through a SUMO1-modification with Nup358 (Matunis et al., 1996; Mahajan et al., 1997). Continuous nuclear transport is dependent on RanGTP-hydrolysis for disassembly of recycling import receptors and/or release of the export complex from the NPC (Moore et al., 1993; Schlenstedt et al., 1995, also reviewed in Fried et al., 2003). Therefore, the localization of RanGAP1 in close proximity to the docking site of transport complexes on their way into/out of the nucleus might increase the efficiency of transport. As depletion of Nup358 leads to loss of NPC-associated RanGAP1 (Hutten et al., 2008), the cytoplasmic accumulation of certain substrates in Nup358-depleted cells could possibly result from the lack of pore-associated RanGAP1.

Exogenous full-length Nup358 can associate with SUMO-modified RanGAP1 (RanGAP1*SUMO1), and its expression relocalizes RanGAP1 back to the NPC. Hence, the rescue effect on the substrates in Nup358-depleted cells might be derived only indirectly by Nup358, but directly by RanGAP1. However, in yeast cells, the localization of RanGAP1 (rna1p) to the NPC is not needed for nuclear transport. Moreover, depletion of 
pore-associated RanGAP1 with a soluble Nup358 fragment did not inhibit nuclear import of cNLS- and M9-cargos in HeLa cells (Hutten et al., 2008; Hutten et al., 2009).

Transfection of Nup358 fragments in a Nup358-depleted background now gave us the opportunity to investigate the role of RanGAP1 in its contribution to nuclear transport in detail. Strikingly, Nup358 aa 1-2448, which does not associate anymore with RanGAP1*SUMO1, can efficiently rescue nuclear localization of DBC-1, HIV-1 Rev and the cNLS- and M9-reporter. Therefore, their nuclear import does not depend on Nup358-associated RanGAP1. Hence, the soluble form is sufficient to stimulate Ran's hydrolysis activity on recycling importin $\alpha$, importin $\beta$ and transportin, corroborating our previous results (Hutten et al., 2008; Hutten et al., 2009).

Why does RanGAP1*SUMO1 localize to the NPC and form a stable complex with Nup358 if not required for RanGTP-hydrolysis, and hence for nucleocytoplasmic transport (Mahajan et al., 1997; Matunis et al., 1998)? In plants, RanGAP1 is anchored to the outer nuclear membrane by a SUMO-independent mechanism (Rose et al., 2001) arguing for a specific role at the NE. Maybe the association in interphase is a prerequisite for the interaction and function of the complex in mitosis.

Further, Nup358 possesses E3 ligase activity (Pichler et al., 2002), and could therefore modify targets on their way into the nucleus pointing to a regulatory task of the nucleoporin. However, involvement of Nup358 as SUMO E3 ligase in the transport machinery of DBC-1, HIV-1 Rev and the reporter proteins analyzed here can be excluded as the Nup358 fragments that are sufficient to mediate nuclear uptake, do not contain the catalytically domain and, hence, cannot modify possible targets. Further, SUMO E3 ligase activity of Nup358 towards DDX43, DDX59 and Sox 6 can be excluded because in vivo and in vitro approaches did not reveal any SUMO-modification (data not shown). In this context, no in vivo targets of the E3 ligase Nup358 except for Topoisomerase II $\alpha$ (Dawlaty et al., 2008) are known so far.

Together, neither Nup358-associated RanGAP1 nor the SUMO E3 ligase activity of the nucleoporin seem to play a major role in nuclear import of the proteins analyzed so far in this work. 


\subsubsection{Distinct domains of Nup358 mediate nuclear import of different import pathways}

\subsubsection{The N-terminal part of Nup358 is sufficient for nuclear transport of cargos using the importin $\alpha / \beta$ pathway}

With the help of the Nup358 fragments deleted of certain domains, it was possible to identify the import mediating domains of Nup358. The N-terminal third of Nup358 is sufficient to mediate nuclear import of DBC-1 and the shuttling reporter protein NES-GFP 2 -cNLS. Here, rescue of nuclear import with the fragment aa 1-1306 containing one Ran-binding domain is slightly more efficient than with fragment aa 1-1170 lacking the RanBDs altogether. Thus, soluble RanBP1 can completely compensate for the loss of the four RanBDs of Nup358, which has already been suggested (Hutten et al., 2008). RanBP1 contains a RanBD, and promotes dissociation of RanGTP from the recycling import receptors which enables RanGAP1 activity on GTP-hydrolysis (Beddow et al., 1995; Ouspenski et al., 1995; Bischoff et al., 1995).

The obtained results point towards a specific interaction of DBC-1 with the N-terminal part of Nup358. Soluble Nup358 fragments like fragment aa 806-1170 can inhibit nuclear import of DBC-1, but nuclear uptake of other proteins like UTF1 and $\mathrm{GFP}_{2}$-NLS. Further, DBC-1 can associate with these soluble fragments in an NTR-independent way, suggesting a different interaction mechanism with the NPC as most transport cargos bind to nucleoporins through NTRs.

The strong decrease to mediate nuclear import of the fragment aa 1-1133 is remarkable that just lacks 40 more amino acids compared to fragment aa 1-1170. One obvious difference concerning nuclear transport is a FG-repeat at aa 1141. However, a respective mutant, aa 1-1306 F1141A, was still able to mediate nuclear import of DBC-1, which has been also validated for fragment aa 1-1170 F1141A (data not shown). They still contain two FG-repeats like fragment aa 1-1133, and the amount of bound importin $\beta$ did not change with the additional FG-repeat. In this context, deletion of all three FG-repeats in the fragment aa 1-1170 should be performed. The rescue ability of such a construct will give further insight into the nature/mode of interaction between cargo and Nup358. 


\subsubsection{Import of transportin cargos cannot be mediated by the $\mathrm{N}$-terminal part of Nup358}

Besides the importin $\alpha / \beta$-dependent cargos, also transportin substrates have been analyzed concerning the domain of Nup358 mediating their nuclear import. In contrast to DBC-1 and the cNLS-reporter protein, fragment aa 1-1306 is not sufficient for import of the M9-cargo and HIV-1 Rev (Figure 4-1). A longer fragment, aa 1-2448, promotes import of all substrates. Interestingly, the additional 1000 amino acids include the zinc finger motif, RanBD2 and nine FG-repeats. Further analysis of the fragments aa 1-1810 and aa 1-2148 will reveal the exact domain(s) promoting nuclear import of both transportin cargos, and will provide more information about the underlying molecular mechanism of this pathway.

An important contribution for understanding the role of Nup358 in nuclear transport will be the in depth analysis of the identified domains that are sufficient or required for nuclear import of the cargos. Here, the number and/or kind of FG-repeats might also be crucial as there are several distinct classes (Wente et al., 1994). Nup358 contains 11 FG- and 11 FXFG-repeats (Figure 4-1), and different NTRs prefer distinct FG-repeats (Marelli et al., 1998; Damelin et al., 2000). Perhaps, importin $\beta$ prefers the FG-repeats located in the N-terminus whereas transportin rather binds to the FGs in the middle part of Nup358. Strikingly, between aa 1350-2012 there are mostly FXFG-repeats in form of FKFG which might point to a specific transportin characteristic feature. Exchange of certain FG-motifs against others might lead to more insight into the interaction between NTR and Nup358.

In addition, similar approaches like for DBC-1 will be performed for the NLS- and M9-cargo as well as for HIV-1 Rev (Figures 3-21 and 3-22). Import inhibition and immunoprecipitations with the soluble Nup358 fragments will reveal more insight into the exact mode of interaction. Based on the specific differences in the rescue ability of distinct Nup358 truncations, the transportin cargos might interact with Nup358 domains located more in the middle part of Nup358 whereas NES-GFP ${ }_{2}$-CNLS could probably interact with the $\mathrm{N}$-terminal fragments. 
In summary, the N-terminal third of Nup358 can mediate nuclear import for substrates using the importin $\alpha / \beta$ pathway very efficiently and comparable to full-length. However, the same fragment cannot promote nuclear import of transportin cargos like HIV-1 Rev. This finding is rather interesting as it points to different requirements of the distinct import pathways regarding the domains of Nup358. The anchor fragment aa 1-1133 lacking 40 amino acids cannot mediate nuclear import at all, as seen for other Nup358-dependent proteins like DDX43 and DDX59 (Fischer, 2009).
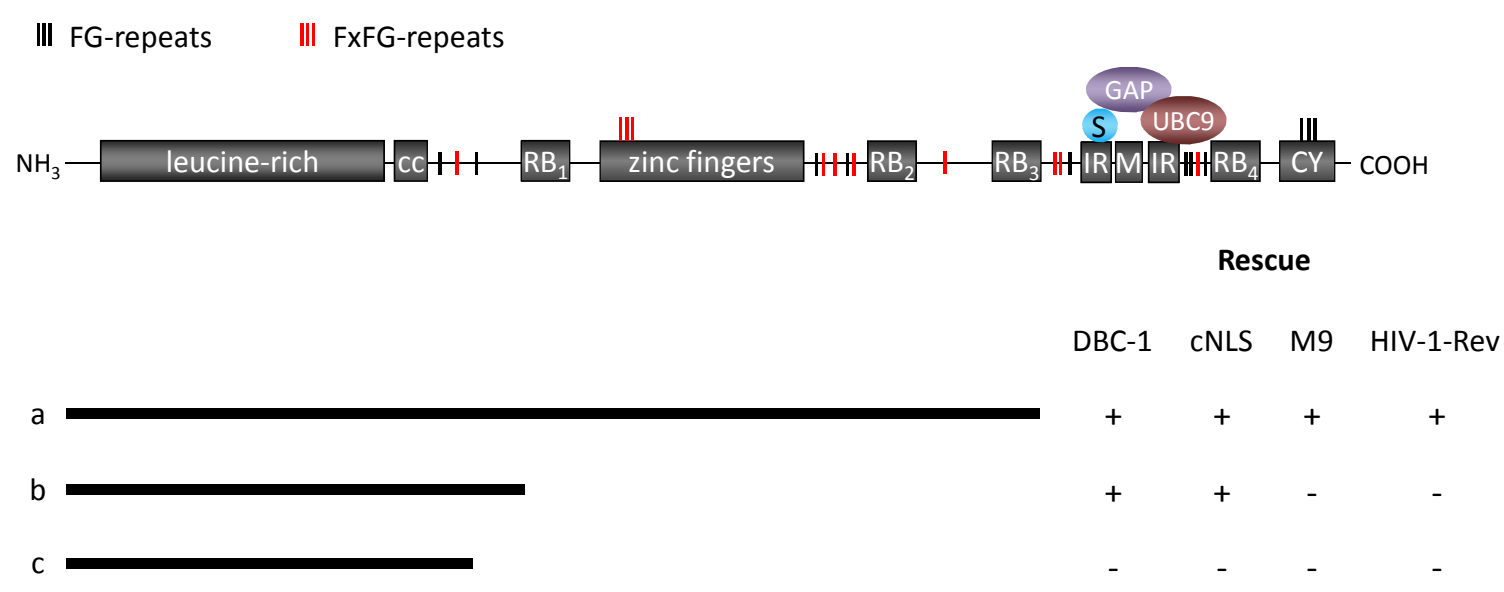

Figure 4-1: Distinct transport pathways require different domains of Nup358 in their nuclear import.

The different classes of FG-repeats are indicated in the schematic description of Nup358. Black dashes are sole FG-repeats, and FXFG-repeats are shown in red. Black bars indicate different Nup358 truncations used in the rescue experiments to compensate for the loss of Nup358. The fragments aa 1-2448 (a), aa 1-1170 (b)

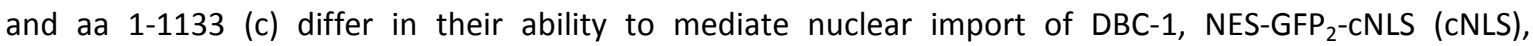
$\mathrm{GR}_{2}-\mathrm{GFP}_{2}-\mathrm{M9}$ (M9) and HIV-1 Rev with + standing for efficient import and - for failed import of the substrates. 


\subsection{Interactions between cargo and the NPC can occur by different mechanisms}

\subsubsection{The $\mathbf{N}$-terminal part of Nup358 functions as an assembly platform for transport complexes with importin $\alpha / \beta$}

The experiments performed in this work clearly show that the $\mathrm{N}$-terminal part of Nup358 lacking any RanBD and associated RanGAP1 can fulfill the function as binding platform for the assembly of new import complexes using the importin $\alpha / \beta$ pathway. As already shown, the soluble factors RanGAP1 and RanBP1 are sufficient to hydrolyze RanGTP of the recycling RanGTP/importin $\beta$ complex in close proximity to the NPC (Hutten et al., 2008). Free importin $\beta$ is enriched by association with one of the three $\mathrm{N}$-terminal FG-repeats in Nup358. This NPC-associated importin $\beta$ can then bind to importin $\alpha$, which in turn has bound the NLS-cargo, and mediates efficient import. Hence, depletion of Nup358 leads to reduced import rates of the cargo because importin $\beta$ becomes rate-limiting (Hutten et al., 2008).

However, it is still unclear where exactly the new import complex is formed. For termination of the import cycle, the importin $\beta /$ RanGTP complex needs to be disassociated in the cytoplasm. RanBD1 and the Ran-binding domains of Nup358 increase the off-rate of RanGTP by stimulating RanGAP1 activity (Bischoff et al., 1995, also reviewed in Macara, 2001; Fried et al., 2003). Moreover, importin $\alpha$ is required to facilitate this process (Bischoff et al., 1997). Hence, the importin $\alpha / \beta$ complex stays in close proximity to the NPC, probably bound to Nup358 waiting for an import cargo to bind which corroborates the function of Nup358 as binding platform for the disassembly of recycling import receptors and the assembly of new import complexes (Figure 4-2). 




Figure 4-2: The N-terminal part of Nup358 functions as a platform for the assembly/disassembly of importin $\alpha / \beta$ transport complexes.

Importin $\alpha / \beta$-dependent nuclear import begins with export of recycling importin $\beta(\beta)$ from the nucleus to the cytoplasm in a complex with RanGTP (T). Soluble RanGAP1 (GAP), RanBP1 (BP1) and importin $\alpha$ can stimulate the hydrolysis of RanGTP to RanGDP (D) in close proximity to the NPC. Upon dissociation of Ran, importin $\alpha$ and importin $\beta$ might bind to the N-terminal part of Nup358, and an import substrate (NLS) can bind to importin $\alpha$. Together, Nup358 concentrates importin $\alpha$ and importin $\beta$ at the nuclear pore. Inside the nucleus, binding of RanGTP to the import complex facilitates its disassembly. See text for more detail.

\subsubsection{Receptor-independent interaction between DBC-1 and Nup358}

Nuclear import of DBC-1 can be promoted by the same region of Nup358 as NES-GFP $P_{2}-C_{N L S}$. However, it seems that the underlying mechanisms are distinct for the two cargos. Whereas the concentration of importin $\beta$ is the rate-limiting factor for NES-GFP 2 -cNLS in Nup358-depleted cells, and overexpression of the NTR can overcome the impaired import (Hutten et al., 2008), overexpression of the importins cannot compensate for the loss of the nucleoporin for import of DBC-1 (Figure 3-11). This suggests that neither importin $\alpha$, importin $\beta$, transportin, nor importin 7 seem to be the only rate-limiting factors for DBC-1 upon Nup358 depletion, and argues for a direct 
interaction between DBC-1 and Nup358 or the involvement of additional factors. Indeed, DBC-1 interacts specifically with Nup358 within the region aa 1000-1170 in the absence and presence of RanQ69L which argues for an NTR-independent association. Moreover, nuclear import of DBC-1 could be inhibited by this fragment as well.

Targeting of DBC-1 to the N-terminal part of Nup358 might occur on its own mediated by an interaction between DBC-1 and the nucleoporin. Alternatively, DBC-1 could be recognized in the cytoplasm by a soluble factor (mediator) that targets the protein to the NPC. There it might interact with the region spanning amino acids 1000-1170, waiting for importin $\alpha / \beta$. Recycling importins from the nucleus may not leave the area around the NPC, and probably interact with the N-terminal part of Nup358. Recognition of DBC-1 or the DBC-1/mediator complex is followed by translocation of the import complex through the NPC. Therefore, Nup358 still functions as binding platform, and DBC-1 interacts with Nup358 either directly or indirectly, but independently of NTRs. Recognition of importin $\alpha$ could occur at Nup358. Hence, after depletion of Nup358, the formation platform of the import complex is missing, and DBC-1 cannot be bound efficiently by importin $\alpha$. It needs further analysis to finally state the precise function.

It seems that nuclear import of DBC-1 and other proteins is controlled and regulated by several factors like Nup358, importin $\alpha / \beta$ or other NTRs and a possible mediator. For both proteins, DBC-1 and Nup358, it was suggested to play a role in tumorigenesis; now it will be interesting to investigate if there might be a link between these two proteins in the development of cancer and if Nup358 might be a important regulator of DBC-1 and putative other proteins involved in this cellular process.

\subsubsection{Import cargo and NTR can both contribute to nuclear pore interaction}

During their translocation across the NE, nuclear transport cargos need to interact with components of the NPC. Most of these associations occur indirectly, mediated by NTRs and FG-repeats of nucleoporins. However, nuclear import can also be carrier- and energy-independent by a direct interaction between cargo and nucleoporin. For example, 
the HIV-1 integrase (Woodward et al., 2009) or unphosphorylated Stat1 (Marg et al., 2004) interact with the FG-rich C-terminus of Nup153. An intermediate step might be the way DBC-1 is transported. DBC-1 interacts with Nup358 independently of NTRs. The translocation step through the NPC is mediated by importin $\alpha / \beta$. Figure $4-3$ describes the different ways of translocation through the NPC.

There is increasing evidence that different nuclear transport pathways exist in which distinct nucleoporins are involved in dependency of certain NTRs and cargos whereas this Nup does not affect nuclear import of other proteins. For example, import of the glucocorticoid receptor/HSP90 complex requires p62 (Echeverria et al., 2009), and nuclear uptake of HSP70 depends on Nup155 (Zhang et al., 2008). The transcription factor NFAT cannot be exported anymore after depletion of Nup214 (Hutten et al., 2006). Altogether, there exist much more different transport mechanisms through the NPC as assumed so far. It needs further investigations to reveal the features of these specific transport pathways, for example why only certain substrates are affected and what specific roles the nucleoporins might have. 
A $\quad$ B $\quad$ C

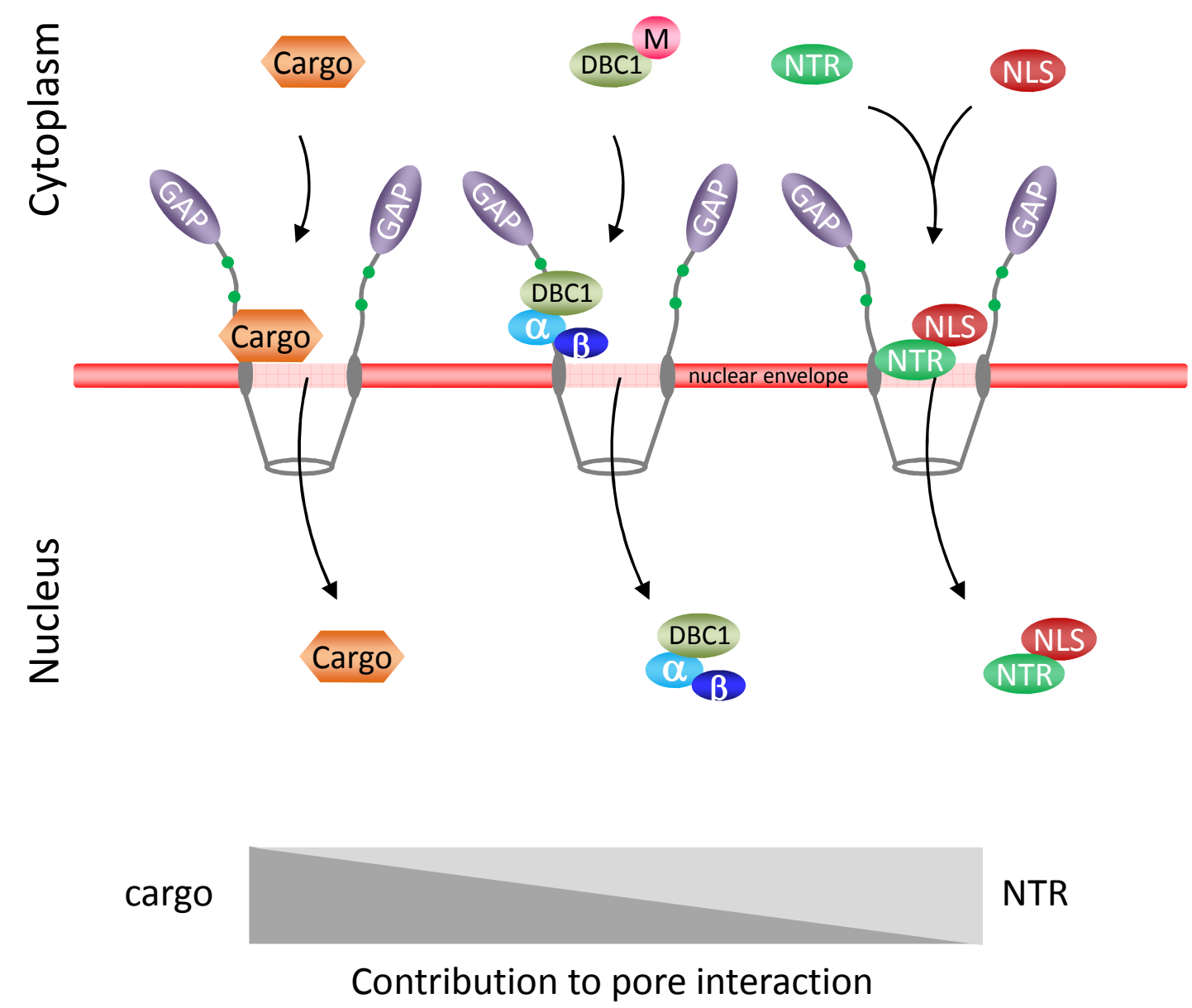

Figure 4-3: Interactions between cargo and the NPC can occur by different mechanisms.

A: Specific cargos like the HIV-1 integrase (Woodward et al., 2009) or unphosphorylated Stat1 (Marg et al., 2004) interact directly with nucleoporins and are transported to the nucleus in a carrier- and energy-independent manner. The association of cargo (any protein) to the NPC is random, and does not describe a certain nucleoporin. B: DBC-1 and other proteins may bind to Nup358 in an NTR-independent manner. Targeting to the NPC might be performed by a mediator (M). Translocation through the NPC is promoted by importin $\alpha / \beta$. This cargo/Nup interaction could contribute to nuclear transport of certain substrates. C: Translocation of NLS-cargos is exclusively mediated by an interaction between NTRs and FG-repeats of certain nucleoporins. Grey box indicates the decreasing (dark grey: cargo) or increasing (light grey: NTR) contribution to pore interaction. See text for more details. 


\subsection{Outlook}

In the course of this work, the cargo/Nup358 specific transport mechanism was analyzed in detail. The majority of proteins is not affected by depletion of Nup358 from the NPC; a small subset however fails to be imported efficiently, pointing towards specific mechanisms and import requirements of these proteins with respect to their docking sites at the NE.

Nuclear import of the importin $\alpha / \beta$ cargos DBC-1 and NES-GFP 2 -CNLS can be mediated by an N-terminal Nup358 fragment, a fragment which is just 40 aa shorter, is not sufficient to promote nuclear import of these substrates. This striking difference in promoting nuclear uptake would be interesting to analyze in more detail, and will hopefully give insight into the interaction between cargo and Nup358. Is the region of interest alone sufficient to mediate nuclear import or is a neighboring sequence necessary to extend the interaction platform for binding partners? Swapping of domains and/or different kinds of FG-repeats in the full-length Nup358 construct might be helpful to shed more light on the type of interaction. The ability of full-length Nup358 deleted specifically of these additional 40 amino acids can also be analyzed to promote nuclear import in Nup358-depleted cells.

To further characterize the exact mechanism for the interaction between cargo and Nup358, the soluble Nup358 fragments need to be tested in the context of the NLS-cargo, and interaction assays will be performed as done for DBC-1. To get more information about a possible mediator, binding assays with recombinant proteins are inevitable to make a clear statement about a direct/indirect interaction. In case a mediator is involved, DBC-1 can be immunoprecipitated, and possible interaction partners might be analyzed by mass spectrometry.

With respect to transportin cargos, the exact region between amino acids 1306 and 2448 to mediate nuclear import needs to be defined in rescue experiments with respective Nup358 truncations. Interaction assays similarly to DBC-1 need to be performed with Nup358 fragments that span the region of interest required for nuclear import of the M9-cargo and HIV-1 Rev. It cannot be excluded that other soluble proteins are involved in the interaction between cargo and Nup358. 
Are other import pathways besides the importin $\alpha / \beta$ and transportin involved in Nup358-dependency? Here, the import mechanisms of other Nup358-dependent proteins have to be characterized in more detail. Moreover, rescue experiments with the Nup358 truncations must be performed to define the regions within Nup358 that mediate nuclear import of these substrates. So far, we only analyzed the localization of transiently transfected proteins in Nup358-depleted cells. Hence, it will also be interesting to look for endogenous proteins in Nup358-depleted cells, for example DBC-1.

As Nup358 is a multi-domain protein with different functions, depletion of Nup358 can also affect possibly other roles of Nup358 like the Sumo E3 ligase activity for sumoylation. A very important and powerful tool to shed more light on the complex role of Nup358 in other functions are the constructs expressing either full-length Nup358 or truncations which have been obtained in the course of this work.

Together, we hope to gain more insight into the transport pathways of some interesting proteins like DBC-1 and HIV-1 Rev and, most importantly in this context, into the unexpected role of Nup358 in transport. 


\section{REFERENCES}

Adam, S.A., Marr, R.S., and Gerace, L. 1990. Nuclear protein import in permeabilized mammalian cells requires soluble cytoplasmic factors. J. Cell Biol. 111:807-816.

Akey, C.W., and Radermacher, M. 1993. Architecture of the Xenopus nuclear pore complex revealed by three-dimensional cryo-electron microscopy. J. Cell Biol. 122:1-19.

Alber, F., Dokudovskaya, S., Veenhoff, L.M., Zhang, W., Kipper, J., Devos, D., Suprapto, A., Karni-Schmidt, O., Williams, R., Chait, B.T., Rout, M.P., and Sali, A. 2007a. Determining the architectures of macromolecular assemblies. Nature. 450:683-94.

Alber, F., Dokudovskaya, S., Veenhoff, L.M., Zhang, W., Kipper, J., Devos, D., Suprapto, A., Karni-Schmidt, O., Williams, R., Chait, B.T., Sali, A., and Rout, M.P. 2007b. The molecular architecture of the nuclear pore complex. Nature. 450:695-701.

Andrade, M.A., and Bork, P. 1995. HEAT repeats in the Huntington's disease protein. Nat Genet. 11:115-6.

Andrade, M.A., Petosa, C., O'Donoghue, S.I., Muller, C.W., and Bork, P. 2001. Comparison of ARM and HEAT protein repeats. J Mol Biol. 309:1-18.

Arnold, M., Nath, A., Hauber, J., and Kehlenbach, R.H. 2006. Multiple importins function as nuclear transport receptors for the Rev protein of human immunodeficiency virus type 1. J. Biol. Chem. 281:20883-20890. Epub 2006 May 16.

Arts, G.J., Fornerod, M., and Mattaj, I.W. 1998. Identification of a nuclear export receptor for tRNA. Curr Biol. 8:305-14.

Aslanukov, A., Bhowmick, R., Guruju, M., Oswald, J., Raz, D., Bush, R.A., Sieving, P.A., Lu, X., Bock, C.B., and Ferreira, P.A. 2006. RanBP2 modulates Cox11 and hexokinase I activities and haploinsufficiency of RanBP2 causes deficits in glucose metabolism. PLoS Genet. 2:e177. Epub 2006 Sep 1.

Ausubel, F.M., Brent, R., Kingston, R.E., Moore, D.D., Seidman, J.G., Smith, J.A., and Struhl, K. 1994. Current protocols in molecular biology. New York: Greene Publishing Associates and Wiley-Interscience.

Baake, M., Bauerle, M., Doenecke, D., and Albig, W. 2001. Core histones and linker histones are imported into the nucleus by different pathways. Eur. J. Cell Biol. 80:669-677.

Bannasch, D., Mehrle, A., Glatting, K.H., Pepperkok, R., Poustka, A., and Wiemann, S. 2004. LIFEdb: a database for functional genomics experiments 
integrating information from external sources, and serving as a sample tracking system. Nucleic Acids Res. 32:D505-8.

Beddow, A.L., Richards, S.A., Orem, N.R., and Macara, I.G. 1995. The Ran/TC4 GTPase-binding domain: identification by expression cloning and characterization of a conserved sequence motif. Proc Natl Acad Sci U S A. 92:3328-32.

Bernad, R., van der Velde, H., Fornerod, M., and Pickersgill, H. 2004. Nup358/RanBP2 attaches to the nuclear pore complex via association with Nup88 and Nup214/CAN and plays a supporting role in CRM1-mediated nuclear protein export. Mol. Cell. Biol. 24:2373-2384.

Birnboim, H.C., and Doly, J. 1979. A rapid alkaline extraction procedure for screening recombinant plasmid DNA. Nucleic Acids Res. 7:1513-23.

Bischoff, F.R., and Görlich, D. 1997. RanBP1 is crucial for the release of RanGTP from importin $\beta$-related nuclear transport factors. FEBS Lett. 419:249-254.

Bischoff, F.R., Klebe, C., Kretschmer, J., Wittinghofer, A., and Ponstingl, H. 1994. RanGAP1 induces GTPase activity of nuclear Ras-related Ran. Proc Natl Acad Sci U S A. 91:2587-2591.

Bischoff, F.R., Krebber, H., Smirnova, E., Dong, W., and Ponstingl, H. 1995. Co-activation of Ran GTPase and inhibition of GTP-dissociation by RanGTP-binding protein RanBP1. EMBO (Eur. Mol. Biol. Organ.) J. 14:705-715.

Bischoff, F.R., and Ponstingl, H. 1991a. Catalysis of guanine nucleotide exchange on Ran by the mitotic regulator RCC1. Nature. 354:80-82.

Bischoff, F.R., and Ponstingl, H. 1991b. Mitotic regulator protein RCC1 is complexed with a nuclear ras-related polypeptide. Proc Natl Acad Sci USA. 88:10830-10834.

Bischoff, F.R., Scheffzek, K., and Ponstingl, H. 2002. How Ran is regulated. Results Probl Cell Differ. 35:49-66.

Black, B.E., Holaska, J.M., Levesque, L., Ossareh-Nazari, B., Gwizdek, C., Dargemont, C., and Paschal, B.M. 2001. NXT1 is necessary for the terminal step of Crm1-mediated nuclear export. J Cell Biol. 152:141-55.

Black, B.E., Levesque, L., Holaska, J.M., Wood, T.C., and Paschal, B.M. 1999. Identification of an NTF2-related factor that binds RanGTP and regulates nuclear protein export. Mol Cell Biol. 19:8616-24.

Bonifaci, N., Moroianu, J., Radu, A., and Blobel, G. 1997. Karyopherin $\beta 2$ mediates nuclear import of a mRNA-binding protein. Proc Natl Acad Sci U S A. 94:5055-60.

Calado, A., Treichel, N., Müller, E.C., Otto, A., and Kutay, U. 2002. Exportin 5-mediated nuclear export of eukaryotic elongation factor 1A and tRNA. Embo J. 21:6216-24. 
Charneau, P., Mirambeau, G., Roux, P., Paulous, S., Buc, H., and Clavel, F. 1994. HIV-1 reverse transcription. A termination step at the center of the genome. J. Mol. Biol. 241:651-662.

Chi, N.C., Adam, E.J., and Adam, S.A. 1995. Sequence and characterization of cytoplasmic nuclear protein import factor p97. J Cell Biol. 130:265-74.

Chi, N.C., Adam, E.J., Visser, G.D., and Adam, S.A. 1996. RanBP1 stabilizes the interaction of Ran with p97 nuclear protein import. J Cell Biol. 135:559-69.

Chi, N.C., and Adam, S.A. 1997. Functional domains in nuclear import factor p97 for binding the nuclear localization sequence receptor and the nuclear pore. Mol. Biol. Cell. 8:945-956.

Chook, Y.M., and Blobel, G. 1999. Structure of the nuclear transport complex karyopherins $\beta 2$ Ran x GppNHp. Nature. 399:230-7.

Chook, Y.M., and Blobel, G. 2001. Karyopherins and nuclear import. Curr Opin Struct Biol. 11:703-15.

Chook, Y.M., Jung, A., Rosen, M.K., and Blobel, G. 2002. Uncoupling Kap $\beta 2$ substrate dissociation and Ran-binding. Biochemistry. 41:6955-66.

Cingolani, G., Bednenko, J., Gillespie, M.T., and Gerace, L. 2002. Molecular basis for the recognition of a nonclassical nuclear localization signal by importin $\beta$. Mol. Cell. 10:1345-1353.

Cingolani, G., Petosa, C., Weis, K., and Müller, C.W. 1999. Structure of importin $\beta$ bound to the IBB-domain of importin $\alpha$. Nature. 399:221-229.

Clarkson, W.D., Kent, H.M., and Stewart, M. 1996. Separate binding sites on nuclear transport factor 2 (NTF2) for RanGDP and the phenylalanine-rich repeat regions of nucleoporins p62 and Nsp1p. J Mol Biol. 263:517-24.

Coller, J., and Parker, R. 2005. General translational repression by activators of mRNA decapping. Cell. 122:875-86.

Conti, E., and Kuriyan, J. 2000. Crystallographic analysis of the specific yet versatile recognition of distinct nuclear localization signals by karyopherin $\alpha$. Structure. 8:329-38.

Conti, E., Uy, M., Leighton, L., Blobel, G., and Kuriyan, J. 1998. Crystallographic analysis of the recognition of a nuclear localization signal by the nuclear import factor karyopherin a. Cell. 94:193-204.

Coutavas, E., Ren, M., Oppenheim, J.D., D'Eustachio, P., and Rush, M.G. 1993. Characterization of proteins that interact with the cell cycle regulatory protein Ran/TC4. Nature. 366:585-587. 
Cronshaw, J.M., Krutchinsky, A.N., Zhang, W., Chait, B.T., and Matunis, M.J. 2002. Proteomic analysis of the mammalian nuclear pore complex. J Cell Biol. 158:91527.

D'Angelo, M.A., and Hetzer, M.W. 2008. Structure, dynamics and function of nuclear pore complexes. Trends Cell Biol. 18:456-66. Epub 2008 Sep 9.

Damelin, M., and Silver, P.A. 2000. Mapping interactions between nuclear transport factors in living cells reveals pathways through the nuclear pore complex. Mol Cell. 5:133-40.

Dawlaty, M.M., Malureanu, L., Jeganathan, K.B., Kao, E., Sustmann, C., Tahk, S., Shuai, K., Grosschedl, R., and van Deursen, J.M. 2008. Resolution of sister centromeres requires RanBP2-mediated sumoylation of topoisomerase Ila. Cell. 133:103-15.

Dean, K.A., von Ahsen, O., Görlich, D., and Fried, H.M. 2001. Signal recognition particle protein 19 is imported into the nucleus by importin 8 (RanBP8) and transportin. $J$ Cell Sci. 114:3479-3485.

Deane, R., Schafer, W., Zimmermann, H.P., Mueller, L., Görlich, D., Prehn, S., Ponstingl, H., and Bischoff, F.R. 1997. Ran-binding protein 5 (RanBP5) is related to the nuclear transport factor importin $\beta$ but interacts differently with RanBP1. Mol. Cell. Biol. 17:5087-96.

Delphin, C., Guan, T., Melchior, F., and Gerace, L. 1997. RanGTP targets p97 to RanBP2, a filamentous protein localized at the cytoplasmic periphery of the nuclear pore complex. Mol. Biol. Cell. 8:2379-90.

Denning, D.P., Patel, S.S., Uversky, V., Fink, A.L., and Rexach, M. 2003. Disorder in the nuclear pore complex: the FG-repeat regions of nucleoporins are natively unfolded. Proc Natl Acad Sci U S A. 100:2450-5. Epub 2003 Feb 25.

Dingwall, C., Sharnick, S.V., and Laskey, R.A. 1982. A polypeptide domain that specifies migration of nucleoplasmin into the nucleus. Cell. 30:449-458.

Dork, T., Stuhrmann, M., Miller, K., and Schmidtke, J. 1998. Independent observation of SRY mutation I90M in a patient with complete gonadal dysgenesis. Hum Mutat. 11:90-1.

Drivas, G.T., Shih, A., Coutavas, E., Rush, M.G., and D'Eustachio, P. 1990. Characterization of four novel ras-like genes expressed in a human teratocarcinoma cell line. Mol Cell Biol. 10:1793-8.

Echeverria, P.C., Mazaira, G., Erlejman, A., Gomez-Sanchez, C., Piwien Pilipuk, G., and Galigniana, M.D. 2009. Nuclear import of the glucocorticoid receptor/hsp90 complex through the nuclear pore complex is mediated by its interaction with Nup62 and importin $\beta$. Mol Cell Biol. 29:4788-97. 
Eisele, N.B., Frey, S., Piehler, J., Görlich, D., and Richter, R.P. 2010. Ultrathin nucleoporin phenylalanine-glycine repeat films and their interaction with nuclear transport receptors. EMBO Rep. 2010:9.

Englmeier, L., Fornerod, M., Bischoff, F.R., Petosa, C., Mattaj, I.W., and Kutay, U. 2001. RanBP3 influences interactions between CRM1 and its nuclear protein export substrates. EMBO Rep. 2:926-32. Epub 2001 Sep 24.

Englmeier, L., Olivo, J.C., and Mattaj, I.W. 1999. Receptor-mediated substrate translocation through the nuclear pore complex without nucleotide triphosphate hydrolysis. Curr Biol. 9:30-41.

Fanara, P., Hodel, M.R., Corbett, A.H., and Hodel, A.E. 2000. Quantitative analysis of nuclear localization signal (NLS-) importin $\alpha$ interaction through fluorescence depolarization. Evidence for autoinhibitory regulation of NLS binding. J Biol Chem. 275:21218-23.

Feldherr, C., Cole, C., Lanford, R.E., and Akin, D. 1994. The effects of SV40 large T antigen and p53 on nuclear transport capacity in BALB/c 3T3 cells. Exp. Cell Res. 213:164171.

Feldherr, C., Kallenbach, E., and Schultz, N. 1984. Movement of a karyophilic protein through the nuclear pores of oocytes. J. Cell Biol. 99:2216-2222.

Fernandez-Lloris, R., Osses, N., Jaffray, E., Shen, L.N., Vaughan, O.A., Girwood, D., Bartrons, R., Rosa, J.L., Hay, R.T., and Ventura, F. 2006. Repression of Sox 6 transcriptional activity by SUMO modification. FEBS Lett. 580:1215-21.

Fischer, S. 2009. Analyse des Nup358-abhängigen Transports. Diplomarbeit, Mathematisch-Naturwissenschaftliche Fakultät, Ernst-August-Universität, Goettingen.

Fischer, U., Huber, J., Boelens, W.C., Mattaj, I.W., and Lührmann, R. 1995. The HIV-1 Rev activation domain is a nuclear export signal that accesses an export pathway used by specific cellular RNAs. Cell. 82:475-483.

Floer, M., and Blobel, G. 1996. The nuclear transport factor karyopherin $\beta$ binds stoichiometrically to RanGTP and inhibits the Ran GTPase activating protein. $J$ Biol Chem. 271:5313-6.

Fontes, M.R., Teh, T., and Kobe, B. 2000. Structural basis of recognition of monopartite and bipartite nuclear localization sequences by mammalian importin $\alpha$. J Mol Biol. 297:1183-94.

Forler, D., Rabut, G., Ciccarelli, F.D., Herold, A., Kocher, T., Niggeweg, R., Bork, P., Ellenberg, J., and Izaurralde, E. 2004. RanBP2/Nup358 provides a major binding site for NXF1/p15 dimers at the nuclear pore complex and functions in nuclear mRNA export. Mol Cell Biol. 24:1155-67. 
Fornerod, M., Ohno, M., Yoshida, M., and Mattaj, I.W. 1997a. CRM1 is an export receptor for leucine-rich nuclear export signals. Cell. 90:1051-1060.

Fornerod, M., van Deursen, J., van Baal, S., Reynolds, A., Davis, D., Murti, K.G., Fransen, J., and Grosveld, G. 1997b. The human homologue of yeast CRM1 is in a dynamic subcomplex with CAN/Nup214 and a novel nuclear pore component Nup88. EMBO (Eur. Mol. Biol. Organ.) J. 16:807-816.

Frey, S., and Görlich, D. 2007. A saturated FG-repeat hydrogel can reproduce the permeability properties of nuclear pore complexes. Cell. 130:512-23.

Frey, S., Richter, R.P., and Görlich, D. 2006. FG-rich repeats of nuclear pore proteins form a three-dimensional meshwork with hydrogel-like properties. Science. 314:815-7.

Fried, H., and Kutay, U. 2003. Nucleocytoplasmic transport: taking an inventory. Cell. Mol. Life Sci. 60:1659-1688.

Fu, J., Jiang, J., Li, J., Wang, S., Shi, G., Feng, Q., White, E., Qin, J., and Wong, J. 2009. Deleted in breast cancer 1 , a novel androgen receptor (AR) coactivator that promotes AR DNA-binding activity. J Biol Chem. 284:6832-40.

Gasca, S., Canizares, J., De Santa Barbara, P., Mejean, C., Poulat, F., Berta, P., and Boizet-Bonhoure, B. 2002. A nuclear export signal within the high mobility group domain regulates the nucleocytoplasmic translocation of Sox 9 during sexual determination. Proc Natl Acad Sci U S A. 99:11199-204.

Gasteier, J.E., Madrid, R., Krautkramer, E., Schroder, S., Muranyi, W., Benichou, S., and Fackler, O.T. 2003. Activation of the Rac-binding partner FHOD1 induces actin stress fibers via a ROCK-dependent mechanism. J Biol Chem. 278:38902-12. Epub 2003 Jul 10.

Gilchrist, D., Mykytka, B., and Rexach, M. 2002. Accelerating the rate of disassembly of karyopherins/cargo complexes. J Biol Chem. 277:18161-72. Epub 2002 Feb 26.

Goldberg, M.W., and Allen, T.D. 1996. The nuclear pore complex and lamina: threedimensional structures and interactions determined by field emission in-lens scanning electron microscopy. J Mol Biol. 257:848-65.

Gontan, C., Guttler, T., Engelen, E., Demmers, J., Fornerod, M., Grosveld, F.G., Tibboel, D., Görlich, D., Poot, R.A., and Rottier, R.J. 2009. Exportin 4 mediates a novel nuclear import pathway for Sox family transcription factors. J Cell Biol. 185:27-34.

Görlich, D., Dabrowski, M., Bischoff, F.R., Kutay, U., Bork, P., Hartmann, E., Prehn, S., and Izaurralde, E. 1997. A novel class of RanGTP-binding proteins. J Cell Biol. 138:6580. 
Görlich, D., Henklein, P., Laskey, R.A., and Hartmann, E. 1996a. A 41 amino acid motif in importin $\alpha$ confers binding to importin $\beta$ and hence transit into the nucleus. Embo J. 15:1810-1817.

Görlich, D., Kostka, S., Kraft, R., Dingwall, C., Laskey, R.A., Hartmann, E., and Prehn, S. 1995a. Two different subunits of importin cooperate to recognize nuclear localization signals and bind them to the nuclear envelope. Curr. Biol. 5:383-392.

Görlich, D., and Kutay, U. 1999. Transport between the cell nucleus and the cytoplasm. Annu. Rev. Cell. Dev. Biol. 15:607-660.

Görlich, D., and Mattaj, I.W. 1996b. Nucleocytoplasmic transport. Science. 271:1513-8.

Görlich, D., Prehn, S., Laskey, R.A., and Hartmann, E. 1994. Isolation of a protein that is essential for the first step of nuclear protein import. Cell. 79:767-778.

Görlich, D., Vogel, F., Mills, A.D., Hartmann, E., and Laskey, R.A. 1995b. Distinct functions for the two importin subunits in nuclear protein import. Nature. 377:246-8.

Grady, R.M., Starr, D.A., Ackerman, G.L., Sanes, J.R., and Han, M. 2005. Syne proteins anchor muscle nuclei at the neuromuscular junction. Proc Natl Acad Sci U S A. 102:4359-64.

Hagiwara, N., Klewer, S.E., Samson, R.A., Erickson, D.T., Lyon, M.F., and Brilliant, M.H. 2000. Sox 6 is a candidate gene for $\mathrm{p} 100 \mathrm{H}$ myopathy, heart block, and sudden neonatal death. Proc Natl Acad Sci U S A. 97:4180-5.

Hamaguchi, M., Meth, J.L., von Klitzing, C., Wei, W., Esposito, D., Rodgers, L., Walsh, T., Welcsh, P., King, M.C., and Wigler, M.H. 2002. DBC2, a candidate for a tumor suppressor gene involved in breast cancer. Proc Natl Acad Sci U S A. 99:13647-52.

Harreman, M.T., Cohen, P.E., Hodel, M.R., Truscott, G.J., Corbett, A.H., and Hodel, A.E. 2003a. Characterization of the autoinhibitory sequence within the $\mathrm{N}$-terminal domain of importin $\alpha$. J Biol Chem. 278:21361-9.

Harreman, M.T., Hodel, M.R., Fanara, P., Hodel, A.E., and Corbett, A.H. 2003b. The autoinhibitory function of importin $\alpha$ is essential in vivo. J Biol Chem. 278:5854-63.

Hodel, M.R., Corbett, A.H., and Hodel, A.E. 2001. Dissection of a nuclear localization signal. J Biol Chem. 276:1317-25.

Hu, T., Guan, T., and Gerace, L. 1996. Molecular and functional characterization of the p62 complex, an assembly of nuclear pore complex glycoproteins. J. Cell Biol. 134:589-601.

Hutten, S. 2007. Untersuchungen zur Funktion der cytoplasmatischen Nukleoporine Nup214 and Nup358 im nukleocytoplasmatischen Transport. Dissertation zur 
Erlangung des Doktorgrades. Mathematisch-Naturwissenschaftliche Fakultät, Ernst-August-Universität, Goettingen.

Hutten, S., Flotho, A., Melchior, F., and Kehlenbach, R.H. 2008. The Nup358/RanGAP complex is required for efficient importin $\alpha / \beta$-dependent nuclear import. Mol Biol Cell. 19:2300-2310. Epub 2008 Feb 27.

Hutten, S., and Kehlenbach, R.H. 2006. Nup214 is required for CRM1-dependent nuclear protein export in vivo. Mol. Cell. Biol. 26:6772-6785.

Hutten, S., Wälde, S., Spillner, C., Hauber, J., and Kehlenbach, R.H. 2009. The nuclear pore component Nup358 promotes transportin-dependent nuclear import. J Cell Sci. 122:1100-10. Epub 2009 Mar 19.

Jäkel, S., Albig, W., Kutay, U., Bischoff, F.R., Schwamborn, K., Doenecke, D., and Görlich, D. 1999. The importin $\beta$ /importin 7 heterodimer is a functional nuclear import receptor for histone H1. Embo J. 18:2411-2423.

Jäkel, S., and Görlich, D. 1998. Importin $\beta$, transportin, RanBP5 and RanBP7 mediate nuclear import of ribosomal proteins in mammalian cells. Embo J. 17:4491-4502.

Jäkel, S., Mingot, J.M., Schwarzmaier, P., Hartmann, E., and Görlich, D. 2002. Importins fulfill a dual function as nuclear import receptors and cytoplasmic chaperones for exposed basic domains. Embo J. 21:377-386.

Jankowsky, E., Gross, C.H., Shuman, S., and Pyle, A.M. 2001. Active disruption of an RNA protein interaction by a DExH/D RNA helicase. Science. 291:121-5.

Jantzen, H.M., Admon, A., Bell, S.P., and Tjian, R. 1990. Nucleolar transcription factor hUBF contains a DNA-binding motif with homology to HMG-proteins. Nature. 344:830-6.

Joseph, J., and Dasso, M. 2008. The nucleoporin Nup358 associates with and regulates interphase microtubules. FEBS Lett. 582:190-6.

Joseph, J., Liu, S.T., Jablonski, S.A., Yen, T.J., and Dasso, M. 2004. The RanGAP1/RanBP2 complex is essential for microtubule kinetochore interactions in vivo. Curr Biol. 14:611-7.

Joseph, J., Tan, S.H., Karpova, T.S., McNally, J.G., and Dasso, M. 2002. SUMO1 targets RanGAP1 to kinetochores and mitotic spindles. J Cell Biol. 156:595-602. Epub 2002 Feb 18.

Kalderon, D., Richardson, W.D., Markham, A.F., and Smith, A.E. 1984a. Sequence requirements for nuclear location of simian virus 40 large $T$ antigen. Nature. 311:33-8. 
Kalderon, D., Roberts, B.L., Richardson, W.D., and Smith, A.E. 1984b. A short amino acid sequence able to specify nuclear location. Cell. 39:499-509.

Kamachi, Y., Uchikawa, M., and Kondoh, H. 2000. Pairing Sox off: with partners in the regulation of embryonic development. Trends Genet. 16:182-7.

Kang, K.I., Devin, J., Cadepond, F., Jibard, N., Guiochon-Mantel, A., Baulieu, E.E., and Catelli, M.G. 1994. In vivo functional protein-protein interaction: nuclear targeted hsp90 shifts cytoplasmic steroid receptor mutants into the nucleus. Poc. Natl. Acad. Sci. USA. 91:340-344.

Kataoka, N., Bachorik, J.L., and Dreyfuss, G. 1999. Transportin-SR, a nuclear import receptor for SR-proteins. J Cell Biol. 145:1145-52.

Kehlenbach, R.H., Dickmanns, A., Kehlenbach, A., Guan, T., and Gerace, L. 1999. A role for RanBP1 in the release of CRM1 from the nuclear pore complex in a terminal step of nuclear export. J. Cell Biol. 145:645-657.

Kim, J.E., Chen, J., and Lou, Z. 2008. DBC-1 is a negative regulator of Sirt1. Nature. 451:583-6.

Klebe, C., Bischoff, F.R., Ponstingl, H., and Wittinghofer, A. 1995. Interaction of the nuclear GTP-binding protein Ran with its regulatory proteins RCC1 and RanGAP1. Biochemistry. 34:639-47.

Kobe, B. 1999. Autoinhibition by an internal nuclear localization signal revealed by the crystal structure of mammalian importin $\alpha$. Nat Struct Biol. 6:388-97.

Koonin, E.V. 1991. Similarities in RNA helicases. Nature. 352:290.

Kraemer, D., Wozniak, R.W., Blobel, G., and Radu, A. 1994. The human CAN protein, a putative oncogene product associated with myeloid leukemogenesis, is a nuclear pore complex protein that faces the cytoplasm. Proc Natl Acad Sci USA. 91:15191523.

Kudo, N., Matsumori, N., Taoka, H., Fujiwara, D., Schreiner, E.P., Wolff, B., Yoshida, M., and Horinouchi, S. 1999. Leptomycin B inactivates CRM1/exportin 1 by covalent modification at a cysteine residue in the central conserved region. Proc Natl Acad Sci U S A. 96:9112-7.

Kudo, N., Wolff, B., Sekimoto, T., Schreiner, E.P., Yoneda, Y., Yanagida, M., Horinouchi, S., and Yoshida, M. 1998. Leptomycin B inhibition of signal-mediated nuclear export by direct binding to CRM1. Exp Cell Res. 242:540-7.

Kutay, U., Bischoff, F.R., Kostka, S., Kraft, R., and Görlich, D. 1997a. Export of importin $\alpha$ from the nucleus is mediated by a specific nuclear transport factor. Cell. 90:10611071. 
Kutay, U., Izaurralde, E., Bischoff, F.R., Mattaj, I.W., and Görlich, D. 1997b. Dominant-negative mutants of importin $\beta$ block multiple pathways of import and export through the nuclear pore complex. Embo J. 16:1153-63.

Kutay, U., Lipowsky, G., Izaurralde, E., Bischoff, F.R., Schwarzmaier, P., Hartmann, E., and Görlich, D. 1998. Identification of a tRNA-specific nuclear export receptor. Mol. Cell. 1:359-69.

Laemmli, U.K. 1970. Cleavage of structural proteins during the assembly of the head of bacteriophage T4. Nature. 227:680-5.

Lai, M.C., Lin, R.I., and Tarn, W.Y. 2001. Transportin-SR2 mediates nuclear import of phosphorylated SR proteins. Proc Natl Acad Sci U S A. 98:10154-9.

Lange, A., McLane, L.M., Mills, R.E., Devine, S.E., and Corbett, A.H. 2010. Expanding the definition of the classical bipartite nuclear localization signal. Traffic. 11:311-23.

Lee, B.J., Cansizoglu, A.E., Suel, K.E., Louis, T.H., Zhang, Z., and Chook, Y.M. 2006. Rules for nuclear localization sequence recognition by karyopherin $\beta$ 2. Cell. 126:543558.

Lee, G.W., Melchior, F., Matunis, M.J., Mahajan, R., Tian, Q., and Anderson, P. 1998. Modification of Ran GTPase-activating protein by the small ubiquitin-related modifier SUMO1 requires Ubc9, an E2-type ubiquitin-conjugating enzyme homologue. J Biol Chem. 273:6503-7.

Lee, S.J., Sekimoto, T., Yamashita, E., Nagoshi, E., Nakagawa, A., Imamoto, N., Yoshimura, M., Sakai, H., Chong, K.T., Tsukihara, T., and Yoneda, Y. 2003. The structure of importin $\beta$ bound to SREBP-2: nuclear import of a transcription factor. Science. 302:1571-5.

Lefebvre, V., Li, P., and de Crombrugghe, B. 1998. A new long form of Sox 5 (L-Sox 5), Sox 6 and Sox 9 are coexpressed in chondrogenesis and cooperatively activate the type II collagen gene. Embo J. 17:5718-33.

Lei, E.P., and Silver, P.A. 2002. Protein and RNA export from the nucleus. Dev Cell. 2:26172.

Li, Z., Chen, L., Kabra, N., Wang, C., Fang, J., and Chen, J. 2009. Inhibition of SUV39H1 methyltransferase activity by DBC-1. J Biol Chem. 284:10361-6.

Lim, R.Y., Fahrenkrog, B., Koser, J., Schwarz-Herion, K., Deng, J., and Aebi, U. 2007. Nanomechanical basis of selective gating by the nuclear pore complex. Science. 318:640-3. Epub 2007 Oct 4.

Linder, P., Lasko, P.F., Ashburner, M., Leroy, P., Nielsen, P.J., Nishi, K., Schnier, J., and Slonimski, P.P. 1989. Birth of the D-E-A-D box. Nature. 337:121-2. 
Lindsay, M.E., Holaska, J.M., Welch, K., Paschal, B.M., and Macara, I.G. 2001. Ran-binding protein 3 is a cofactor for Crm1-mediated nuclear protein export. J Cell Biol. 153:1391-402.

Lipowsky, G., Bischoff, F.R., Schwarzmaier, P., Kraft, R., Kostka, S., Hartmann, E., Kutay, U., and Görlich, D. 2000. Exportin 4: a mediator of a novel nuclear export pathway in higher eukaryotes. Embo J. 19:4362-71.

Liu, J., Prunuske, A.J., Fager, A.M., and Ullman, K.S. 2003. The COPI complex functions in nuclear envelope breakdown and is recruited by the nucleoporin Nup153. Dev Cell. 5:487-98.

Love, D.C., Sweitzer, T.D., and Hanover, J.A. 1998. Reconstitution of HIV-1 rev nuclear export: independent requirements for nuclear import and export. Proc. Natl. Acad. Sci. USA. 95:10608-10613.

Macara, I.G. 2001. Transport into and out of the nucleus. Microbiol. Mol. Biol. Rev. 65:570-594.

Mahajan, R., Delphin, C., Guan, T., Gerace, L., and Melchior, F. 1997. A small ubiquitin-related polypeptide involved in targeting RanGAP1 to nuclear pore complex protein RanBP2. Cell. 88:97-107.

Marelli, M., Aitchison, J.D., and Wozniak, R.W. 1998. Specific binding of the karyopherin Kap121p to a subunit of the nuclear pore complex containing Nup53p, Nup59p, and Nup170p. J Cell Biol. 143:1813-30.

Marg, A., Shan, Y., Meyer, T., Meissner, T., Brandenburg, M., and Vinkemeier, U. 2004. Nucleocytoplasmic shuttling by nucleoporins Nup153 and Nup214 and CRM1-dependent nuclear export control the subcellular distribution of latent Stat1. J Cell Biol. 165:823-33.

Martelange, V., De Smet, C., De Plaen, E., Lurquin, C., and Boon, T. 2000. Identification on a human sarcoma of two new genes with tumor-specific expression. Cancer Res. 60:3848-55.

Mathieu, M.G., Linley, A.J., Reeder, S.P., Badoual, C., Tartour, E., Rees, R.C., and McArdle, S.E. 2010. HAGE, a cancer/testis antigen expressed at the protein level in a variety of cancers. Cancer Immun. 10:2.

Matsuura, Y., Lange, A., Harreman, M.T., Corbett, A.H., and Stewart, M. 2003. Structural basis for Nup2p function in cargo release and karyopherin recycling in nuclear import. Embo J. 22:5358-69.

Matsuura, Y., and Stewart, M. 2004. Structural basis for the assembly of a nuclear export complex. Nature. 432:872-7. 
Matsuura, Y., and Stewart, M. 2005. Nup50/Npap60 function in nuclear protein import complex disassembly and importin recycling. Embo J. 24:3681-9. Epub 2005 Oct 13.

Mattaj, I.W., and Englmeier, L. 1998. Nucleocytoplasmic transport: the soluble phase. Annu. Rev. Biochem. 67:265-306.

Matunis, M.J., Coutavas, E., and Blobel, G. 1996. A novel ubiquitin-like modification modulates the partitioning of the Ran GTPase-activating protein RanGAP1 between the cytosol and the nuclear pore complex. J Cell Biol. 135:1457-70.

Matunis, M.J., Wu, J., and Blobel, G. 1998. SUMO1 modification and its role in targeting the Ran GTPase-activating protein, RanGAP1, to the nuclear pore complex. J Cell Biol. 140:499-509.

McBurney, M.W., Yang, X., Jardine, K., Hixon, M., Boekelheide, K., Webb, J.R., Lansdorp, P.M., and Lemieux, M. 2003. The mammalian SIR2 $\alpha$ protein has a role in embryogenesis and gametogenesis. Mol Cell Biol. 23:38-54.

Melchior, F., Guan, T., Yokoyama, N., Nishimoto, T., and Gerace, L. 1995a. GTP-hydrolysis by Ran occurs at the nuclear pore complex in an early step of protein import. $J$. Cell Biol. 131:571-81.

Melchior, F., Sweet, D.J., and Gerace, L. 1995b. Analysis of Ran/TC4 function in nuclear protein import. Methods Enzymol. 257:279-291.

Merril, C.R., Dunau, M.L., and Goldman, D. 1981. A rapid sensitive silver stain for polypeptides in polyacrylamide gels. Anal Biochem. 110:201-7.

Mingot, J.M., Kostka, S., Kraft, R., Hartmann, E., and Görlich, D. 2001. Importin 13: a novel mediator of nuclear import and export. Embo J. 20:3685-94.

Moore, J.D., Yang, J., Truant, R., and Kornbluth, S. 1999. Nuclear import of Cdk/cyclin complexes: identification of distinct mechanisms for import of Cdk2/cyclin $E$ and Cdc2/cyclin B1. J Cell Biol. 144:213-24.

Moore, M.S., and Blobel, G. 1993. The GTP-binding protein Ran/TC4 is required for protein import into the nucleus. Nature. 365:661-3.

Mosammaparast, N., and Pemberton, L.F. 2004. Karyopherins: from nuclear transport mediators to nuclear function regulators. Trends Cell Biol. 14:547-56.

Moynihan, K.A., Grimm, A.A., Plueger, M.M., Bernal-Mizrachi, E., Ford, E., Cras-Meneur, C., Permutt, M.A., and Imai, S. 2005. Increased dosage of mammalian Sir2 in pancreatic $\beta$ cells enhances glucose-stimulated insulin secretion in mice. Cell Metab. 2:105-17. 
Mühlhäusser, P., Müller, E.C., Otto, A., and Kutay, U. 2001. Multiple pathways contribute to nuclear import of core histones. EMBO Rep. 2:690-696.

Mullis, K.B. 1990. Target amplification for DNA analysis by the polymerase chain reaction. Ann Biol Clin (Paris). 48:579-82.

Murawala, P., Tripathi, M.M., Vyas, P., Salunke, A., and Joseph, J. 2009. Nup358 interacts with APC and plays a role in cell polarization. J Cell Sci. 122:3113-22.

Nemergut, M.E., Lindsay, M.E., Brownawell, A.M., and Macara, I.G. 2002. Ran-binding protein 3 links Crm1 to the Ran guanine nucleotide exchange factor. J Biol Chem. 277:17385-8. Epub 2002 Apr 3.

Neuhoff, V., Arold, N., Taube, D., and Ehrhardt, W. 1988. Improved staining of proteins in polyacrylamide gels including isoelectric focusing gels with clear background at nanogram sensitivity using Coomassie Brilliant Blue G-250 and R-250. Electrophoresis. 9:255-62.

Niman, H.L., Houghten, R.A., Walker, L.E., Reisfeld, R.A., Wilson, I.A., Hogle, J.M., and Lerner, R.A. 1983. Generation of protein-reactive antibodies by short peptides is an event of high frequency: implications for the structural basis of immune recognition. Proc Natl Acad Sci U S A. 80:4949-53.

Ouspenski, I.I., Mueller, U.W., Matynia, A., Sazer, S., Elledge, S.J., and Brinkley, B.R. 1995. Ran-binding protein-1 is an essential component of the Ran/RCC1 molecular switch system in budding yeast. J. Biol. Chem. 270:1975-1978.

Paine, P.L., Moore, L.C., and Horowitz, S.B. 1975. Nuclear envelope permeability. Nature. 254:109-114.

Pante, N., and Aebi, U. 1993. The nuclear pore complex. J. Cell Biol. 122:977-985.

Paschal, B.M., and Gerace, L. 1995. Identification of NTF2, a cytosolic factor for nuclear import that interacts with nuclear pore complex protein p62. J. Cell Biol. 129:925937.

Patel, S.S., Belmont, B.J., Sante, J.M., and Rexach, M.F. 2007. Natively unfolded nucleoporins gate protein diffusion across the nuclear pore complex. Cell. 129:8396.

Patel, S.S., and Rexach, M.F. 2008. Discovering novel interactions at the nuclear pore complex using bead halo: a rapid method for detecting molecular interactions of high and low affinity at equilibrium. Mol Cell Proteomics. 7:121-31.

Pemberton, L.F., and Paschal, B.M. 2005. Mechanisms of receptor-mediated nuclear import and nuclear export. Traffic. 6:187-198. 
Peters, R. 2009. Translocation through the nuclear pore: Kaps pave the way. Bioessays. 31:466-77.

Pichler, A., Gast, A., Seeler, J.S., Dejean, A., and Melchior, F. 2002. The nucleoporin RanBP2 has SUMO1 E3 ligase activity. Cell. 108:109-20.

Plafker, S.M., and Macara, I.G. 2000. Importin 11, a nuclear import receptor for the ubiquitin-conjugating enzyme, UbcM2. Embo J. 19:5502-13.

Pollard, V.W., Michael, W.M., Nakielny, S., Siomi, M.C., Wang, F., and Dreyfuss, G. 1996. A novel receptor-mediated nuclear protein import pathway. Cell. 86:985-994.

Prunuske, A.J., Liu, J., Elgort, S., Joseph, J., Dasso, M., and Ullman, K.S. 2006. Nuclear envelope breakdown is coordinated by both Nup358/RanBP2 and Nup153, two nucleoporins with zinc finger modules. Mol. Biol. Cell. 17:760-769. Epub 2005 Nov 28.

Pyhtila, B., and Rexach, M. 2003. A gradient of affinity for the karyopherin Kap95p along the yeast nuclear pore complex. J Biol Chem. 278:42699-709.

Radu, A., Moore, M.S., and Blobel, G. 1995. The peptide repeat domain of nucleoporin Nup98 functions as a docking site in transport across the nuclear pore complex. Cell. 81:215-22.

Rehberg, S., Lischka, P., Glaser, G., Stamminger, T., Wegner, M., and Rosorius, O. 2002. Sox 10 is an active nucleocytoplasmic shuttle protein, and shuttling is crucial for Sox 10-mediated transactivation. Mol Cell Biol. 22:5826-34.

Rexach, M., and Blobel, G. 1995. Protein import into nuclei: association and dissociation reactions involving transport substrate, transport factors, and nucleoporins. Cell. 83:683-692.

Ribbeck, K., and Görlich, D. 2001. Kinetic analysis of translocation through nuclear pore complexes. Embo J. 20:1320-1330.

Ribbeck, K., Lipowsky, G., Kent, H.M., Stewart, M., and Görlich, D. 1998. NTF2 mediates nuclear import of Ran. Embo J. 17:6587-6598.

Richardson, A.L., Wang, Z.C., De Nicolo, A., Lu, X., Brown, M., Miron, A., Liao, X., Iglehart, J.D., Livingston, D.M., and Ganesan, S. 2006. X chromosomal abnormalities in basal-like human breast cancer. Cancer Cell. 9:121-32.

Richardson, W.D., Mills, A.D., Dilworth, S.M., Laskey, R.A., and Dingwall, C. 1988. Nuclear protein migration involves two steps: rapid binding at the nuclear envelope followed by slower translocation through nuclear pores. Cell. 52:655-664. 
Robbins, J., Dilworth, S.M., Laskey, R.A., and Dingwall, C. 1988. Two interdependent basic domains in nucleoplasmic nuclear targeting sequence: identification of a class of bipartide nuclear targeting sequence. Cell. 64:615-623.

Rocak, S., and Linder, P. 2004. DEAD-box proteins: the driving forces behind RNA metabolism. Nat Rev Mol Cell Biol. 5:232-41.

Rose, A., and Meier, I. 2001. A domain unique to plant RanGAP is responsible for its targeting to the plant nuclear rim. Proc Natl Acad Sci U S A. 98:15377-82.

Rothbauer, U., Zolghadr, K., Muyldermans, S., Schepers, A., Cardoso, M.C., and Leonhardt, H. 2008. A versatile nanotrap for biochemical and functional studies with fluorescent fusion proteins. Mol Cell Proteomics. 7:282-9. Epub 2007 Oct 21.

Rout, M.P., Aitchison, J.D., Magnasco, M.O., and Chait, B.T. 2003. Virtual gating and nuclear transport: the hole picture. Trends Cell Biol. 13:622-8.

Rout, M.P., Aitchison, J.D., Suprapto, A., Hjertaas, K., Zhao, Y., and Chait, B.T. 2000. The yeast nuclear pore complex: composition, architecture, and transport mechanism. J. Cell Biol. 148:635-51.

Sabri, N., Roth, P., Xylourgidis, N., Sadeghifar, F., Adler, J., and Samakovlis, C. 2007. Distinct functions of the Drosophila Nup153 and Nup214 FG-domains in nuclear protein transport. J Cell Biol. 178:557-65. Epub 2007 Aug 6.

Saitoh, H., Cooke, C.A., Burgess, W.H., Earnshaw, W.C., and Dasso, M. 1996. Direct and indirect association of the small GTPase Ran with nuclear pore proteins and soluble transport factors: studies in Xenopus laevis egg extracts. Mol Biol Cell. 7:1319-34.

Sakamoto, J., Miura, T., Shimamoto, K., and Horio, Y. 2004. Predominant expression of Sir $2 \alpha$, an NAD-dependent histone deacetylase, in the embryonic mouse heart and brain. FEBS Lett. 556:281-6.

Salina, D., Enarson, P., Rattner, J.B., and Burke, B. 2003. Nup358 integrates nuclear envelope breakdown with kinetochore assembly. J. Cell Biol. 162:991-1001.

Sambrook, J., Fritsch, E.F., and Maniatis, T. 1989. Molecular Cloning: a laboratory manual. Cold Spring Harbor Laboratory, Cold Spring Harbor, N.Y.

Sanger, F., Nicklen, S., and Coulson, A.R. 1977. DNA sequencing with chain-terminating inhibitors. Proc Natl Acad Sci U S A. 74:5463-7.

Schlenstedt, G., Saavedra, C., Loeb, J.D.J., Cole, C.N., and Silver, P.A. 1995. The GTP-bound form of the yeast Ran/TC4 homologue blocks nuclear protein import and appearance of poly(A)+ RNA in the cytoplasm. Proc. Natl. Acad. Sci. USA. 92:225229. 
Shah, S., and Forbes, D.J. 1998a. Separate nuclear import pathways converge on the nucleoporin Nup153 and can be dissected with dominant-negative inhibitors. Curr Biol. 8:1376-86.

Shah, S., Tugendreich, S., and Forbes, D. 1998b. Major binding sites for the nuclear import receptor are the internal nucleoporin Nup153 and the adjacent nuclear filament protein Tpr. J. Cell Biol. 141:31-49.

Sharp, P.A., Sugden, B., and Sambrook, J. 1973. Detection of two restriction endonuclease activities in Haemophilus parainfluenzae using analytical agarose-ethidium bromide electrophoresis. Biochemistry. 12:3055-63.

Sim, H., Argentaro, A., and Harley, V.R. 2008. Boys, girls and shuttling of SRY and Sox 9. Trends Endocrinol Metab. 26:26.

Singh, B.B., Patel, H.H., Roepman, R., Schick, D., and Ferreira, P.A. 1999. The zinc finger cluster domain of RanBP2 is a specific docking site for the nuclear export factor, exportin 1. J. Biol. Chem. 274:37370-8.

Siomi, H., and Dreyfuss, G. 1995. A nuclear localization domain in the hnRNP A1 protein. J Cell Biol. 129:551-60.

Smith, A., Brownawell, A., and Macara, I.G. 1998. Nuclear import of Ran is mediated by the transport factor NTF2. Curr Biol. 8:1403-6.

Song, J., Durrin, L.K., Wilkinson, T.A., Krontiris, T.G., and Chen, Y. 2004. Identification of a SUMO-binding motif that recognizes SUMO-modified proteins. Proc Natl Acad Sci U S A. 101:14373-8.

Stade, K., Ford, C.S., Guthrie, C., and Weis, K. 1997. Exportin 1 (Crm1p) is an essential nuclear export factor. Cell. 90:1041-50.

Starr, D.A. 2007. Communication between the cytoskeleton and the nuclear envelope to position the nucleus. Mol Biosyst. 3:583-9.

Stewart, M. 2007. Molecular mechanism of the nuclear protein import cycle. Nat Rev Mol Cell Biol. 8:195-208. Epub 2007 Feb 7.

Strawn, L.A., Shen, T., Shulga, N., Goldfarb, D.S., and Wente, S.R. 2004. Minimal nuclear pore complexes define FG-repeat domains essential for transport. Nat. Cell Biol. 6:197-206.

Stüven, T., Hartmann, E., and Görlich, D. 2003. Exportin 6: a novel nuclear export receptor that is specific for profiling/actin complexes. Embo J. 22:5928-40.

Sudbeck, P., and Scherer, G. 1997. Two independent nuclear localization signals are present in the DNA-binding high-mobility group domains of SRY and Sox 9. J Biol Chem. 272:27848-52. 
Sukegawa, J., and Blobel, G. 1993. A nuclear pore complex protein that contains zinc finger motifs, binds DNA, and faces the nucleoplasm. Cell. 72:29-38.

Sundararajan, R., Chen, G., Mukherjee, C., and White, E. 2005. Caspase-dependent processing activates the proapoptotic activity of deleted in breast cancer-1 during tumor necrosis factor- $\alpha$-mediated death signaling. Oncogene. 24:4908-20.

Tanner, N.K. 2003. The newly identified Q motif of DEAD-box helicases is involved in adenine recognition. Cell Cycle. 2:18-9.

Tanno, M., Sakamoto, J., Miura, T., Shimamoto, K., and Horio, Y. 2007. Nucleocytoplasmic shuttling of the NAD+-dependent histone deacetylase Sirt1. J Biol Chem. 282:6823-32.

Terry, L.J., and Wente, S.R. 2009. Flexible gates: dynamic topologies and functions for FG-nucleoporins in nucleocytoplasmic transport. Eukaryot Cell. 8:1814-27. Epub 2009 Oct 2.

Tiganis, T., Flint, A.J., Adam, S.A., and Tonks, N.K. 1997. Association of the T-cell protein tyrosine phosphatase with nuclear import factor p97. J Biol Chem. 272:2154821557.

Timney, B.L., Tetenbaum-Novatt, J., Agate, D.S., Williams, R., Zhang, W., Chait, B.T., and Rout, M.P. 2006. Simple kinetic relationships and nonspecific competition govern nuclear import rates in vivo. J Cell Biol. 175:579-93.

Towbin, H., Staehelin, T., and Gordon, J. 1979. Electrophoretic transfer of proteins from polyacrylamide gels to nitrocellulose sheets: procedure and some applications. Proc Natl Acad Sci U S A. 76:4350-4.

Tran, E.J., Bolger, T.A., and Wente, S.R. 2007. SnapShot: nuclear transport. Cell. 131:420.

Trauernicht, A.M., Kim, S.J., Kim, N.H., and Boyer, T.G. 2007. Modulation of estrogen receptor $\alpha$ protein level and survival function by DBC-1. Mol Endocrinol. 21:152636.

Tseng, S.S., Weaver, P.L., Liu, Y., Hitomi, M., Tartakoff, A.M., and Chang, T.H. 1998. Dbp5p, a cytosolic RNA helicase, is required for poly(A)+ RNA export. Embo J. 17:2651-62.

Tuschl, T. 2001. RNA interference and small interfering RNAs. Chembiochem. 2:239-45.

Tuschl, T., Zamore, P.D., Lehmann, R., Bartel, D.P., and Sharp, P.A. 1999. Targeted mRNA degradation by double-stranded RNA in vitro. Genes Dev. 13:3191-7.

Vetter, I.R., Arndt, A., Kutay, U., Görlich, D., and Wittinghofer, A. 1999. Structural view of the Ran-Importin $\beta$ interaction at 2.3 A resolution. Cell. 97:635-646. 
Vilain, E., Achermann, J.C., Eugster, E.A., Harley, V.R., Morel, Y., Wilson, J.D., and Hiort, O. 2007. We used to call them hermaphrodites. Genet Med. 9:65-6.

Wälde, S., and Kehlenbach, R. 2010. The Part and the Whole: functions of nucleoporins in nucleocytoplasmic transport. Trends in Cell Biology. doi:10.1016/j.tcb.2010.05.001

Walther, T.C., Pickersgill, H.S., Cordes, V.C., Goldberg, M.W., Allen, T.D., Mattaj, I.W., and Fornerod, M. 2002. The cytoplasmic filaments of the nuclear pore complex are dispensable for selective nuclear protein import. J. Cell Biol. 158:63-77.

Weis, K. 2003. Regulating access to the genome: nucleocytoplasmic transport throughout the cell cycle. Cell. 112:441-451.

Weis, K., Ryder, U., and Lamond, A.I. 1996. The conserved N-terminal domain of hSRP1 $\alpha$ is essential for nuclear protein import. Embo J. 15:1818-25.

Wen, W., Meinkoth, J.L., Tsien, R.Y., and Taylor, S.S. 1995. Identification of a signal for rapid export of proteins from the nucleus. Cell. 82:463-73.

Wente, S.R., and Blobel, G. 1994. Nup145 encodes a novel yeast glycine-leucine-phenylalanine-glycine (GLFG) nucleoporin required for nuclear envelope structure. J Cell Biol. 125:955-69.

White, H. 1980. A heteroskedasticity-consistent covariance matrix estimator and a direct test for heteroskedasticity. Econometrica. 48:817-838.

Wilson, M., and Koopman, P. 2002. Matching Sox: partner proteins and co-factors of the Sox family of transcriptional regulators. Curr Opin Genet Dev. 12:441-6.

Wohlwend, D., Strasser, A., Dickmanns, A., Doenecke, D., and Ficner, R. 2007. Thermodynamic Analysis of $\mathrm{H} 1$ Nuclear Import: Receptor tuning of importin $\beta$ /importin 7. J Biol Chem. 282:10707-10719. Epub 2007 Jan 26.

Woodward, C.L., Prakobwanakit, S., Mosessian, S., and Chow, S.A. 2009. Integrase interacts with nucleoporin Nup153 to mediate the nuclear import of human immunodeficiency virus type 1. J Virol. 83:6522-33. Epub 2009 Apr 15.

Wu, J., Matunis, M.J., Kraemer, D., Blobel, G., and Coutavas, E. 1995. Nup358, a cytoplasmically exposed nucleoporin with peptide repeats, RanGTP-binding sites, zinc fingers, a cyclophilin A homologous domain, and a leucine-rich region. J. Biol. Chem. 270:14209-13.

Wu, X., Kasper, L.H., Mantcheva, R.T., Mantchev, G.T., Springett, M.J., and van Deursen, J.M. 2001. Disruption of the FG-nucleoporin Nup98 causes selective changes in nuclear pore complex stoichiometry and function. Proc Natl Acad Sci U S A. 98:3191-6. 
Yamada, J., Phillips, J.L., Patel, S., Goldfien, G., Calestagne-Morelli, A., Huang, H., Reza, R., Acheson, J., Krishnan, V.V., Newsam, S., Gopinathan, A., Lau, E.Y., Colvin, M.E., Uversky, V.N., and Rexach, M.F. 2010. A bimodal distribution of two distinct categories of intrinsically-disordered structures with separate functions in FG-nucleoporins. Mol Cell Proteomics.

Yamazumi, Y., Kamiya, A., Nishida, A., Nishihara, A., lemura, S., Natsume, T., and Akiyama, T. 2009. The transmembrane nucleoporin NDC1 is required for targeting of ALADIN to nuclear pore complexes. Biochem Biophys Res Commun. 389:100-4. Epub 2009 Aug 22.

Yaseen, N.R., and Blobel, G. 1997. Cloning and characterization of human karyopherin $\beta 3$. Proc Natl Acad Sci U S A. 94:4451-6.

Yaseen, N.R., and Blobel, G. 1999. Two distinct classes of Ran-binding sites on the nucleoporin Nup-358. Proc Natl Acad Sci U S A. 96:5516-21.

Yokoyama, N., Hayashi, N., Seki, T., Pante, N., Ohba, T., Nishii, K., Kuma, K., Hayashida, T., Miyata, T., Aebi, U., Fukui, M., and Nishimoto, T. 1995. A giant nucleopore protein that binds Ran/TC4. Nature. 376:184-188.

Zhang, X., Chen, S., Yoo, S., Chakrabarti, S., Zhang, T., Ke, T., Oberti, C., Yong, S.L., Fang, F., Li, L., de la Fuente, R., Wang, L., Chen, Q., and Wang, Q.K. 2008. Mutation in nuclear pore component Nup155 leads to atrial fibrillation and early sudden cardiac death. Cell. 135:1017-27.

Zhao, W., Kruse, J.P., Tang, Y., Jung, S.Y., Qin, J., and Gu, W. 2008. Negative regulation of the deacetylase Sirt1 by DBC-1. Nature. 451:587-90. 



\section{APPENDIX}

\section{Figure I}

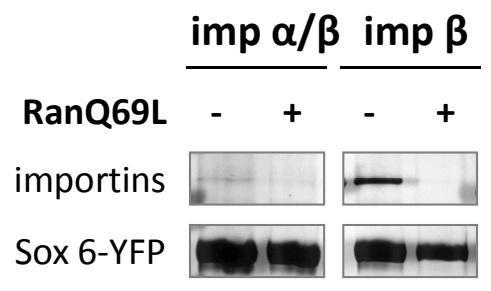

Importin $\boldsymbol{\beta}$ can bind directly to Sox $\mathbf{6}$.

As the SRY protein is transported actively into the nucleus by binding directly to importin $\beta$ (Sudbeck et al., 1997), we also tested interaction of importin $\beta$ to Sox 6 without importin $\alpha$ as an adaptor protein. Therefore, Sox 6-YFP was immunoprecipitated from 293T HEK cells using the GFP-nanotrap and incubated with the importin $\alpha / \beta$ complex or importin $\beta$ alone in the absence $(-)$ or presence $(+)$ of RanQ69L which had been loaded with GTP. Interacting proteins were analyzed by SDS-PAGE, followed by colloidal coomassie staining. 


\section{Figure II}

A

Localization

full-length

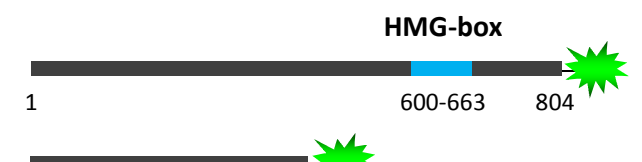

aa 1-455

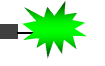

N

aa $456-804$

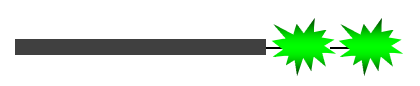

C

aa 456-606

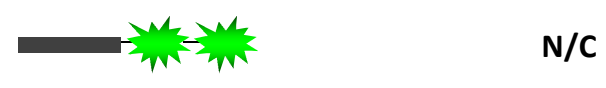

N

aa 456-606

aa 456-606 R600G

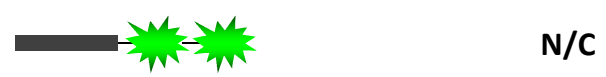

aa 601-701

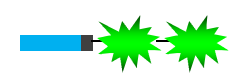

$\mathbf{N}$

aa 601-701

G633A/R635A/

K644A/P646A

aa 601-701

RK614/615AA

633 GSRWKSMSNQEKKQPY 647
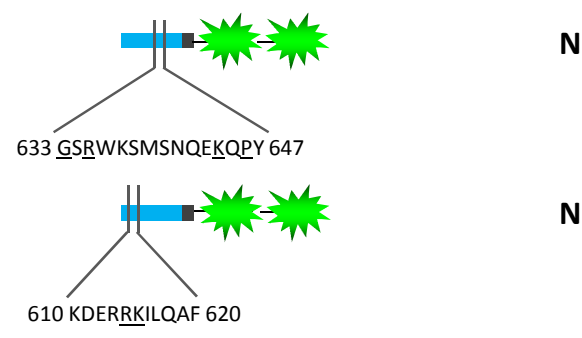

N

aa 702-804

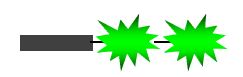

N/C

B

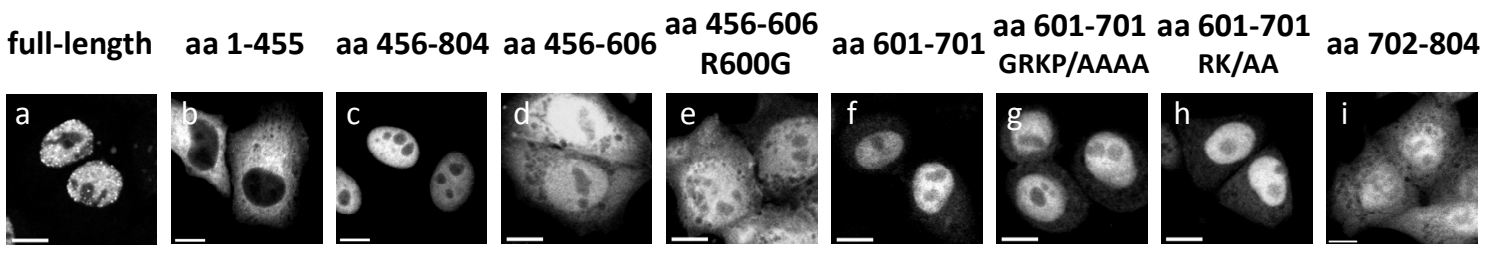


The C-terminal half of Sox 6 contains several NLS.

A: The scheme describes Sox 6 full-length, the N-terminal fragment aa 1-455 and various fragments spanning the C-terminus. Their subcellular localization after transient transfection into HeLa P4 cells is shown as well ( $\mathrm{N}=$ nucleus, $\mathrm{N} / \mathrm{C}=$ equally distributed between nucleus and cytoplasm, $\mathrm{C}=$ cytoplasm). To abolish the interaction of exportin 4 in the fragment aa 456-606, arginine at position 600 was mutated to glycine. The sequence ${ }_{633} \underline{G S R W K S M S N Q E K Q P Y}_{647}$ fulfilled requirements of a putative PY-motif for the interaction with transportin (Lee et al., 2006). Hence, residues 633, 635, 644 and 646 were mutated to alanines in the short fragment aa 601-701. Arginine and lysine in a cNLS-like motif ${ }_{610}$ KDERRKILQAF $_{620}$ were mutated to alanines. Green labeling indicates a GFP-tag (single or double). B: HeLa P4 cells were transiently transfected with plasmids coding for either Sox 6 full-length or fragments. Scale bars: $10 \mu \mathrm{m}$. As the indicated mutations in the Sox fragments did not result in cytoplasmic accumulation, nuclear import of Sox 6 might be mediated by several importins compensating for each other.

\section{Figure III}

1 MSQFKRQRINPLPGGRNFSGTASTSLLGPPPGLLTPPVATELSQNARHLQGGEKQRVFTG

61 IVTSLHDYFGVVDEEVFFQLSVVKGRLPQLGEKVLVKAAYNPGQAVPWNAVKVQTLSNQP

121 LLKSPAPPLLHVAALGQKQGILGAQPQLIFQPHRIPPLFPQKPLSLFQTSHTLHLSHLNR

181 FPARGPHGRLDQGRSDDYDSKKRKQRAGGEPWGAKKPRHDLPPYRVHLTPYTVDSPICDF

241 LELQRRYRSLLVPSDFLSVHLSWLSAFPLSQPFSLHHPSRIQVSSEKEAAPDAGAEPITA

301 DSDPAYSSKVLLLSSPGLEELYRCCMLFVDDMAEPRETPEHPLKQIKFLLGRKEEEAVLV

361 GGEWSPSLDGLDPQADPQVLVRTAIRCAQAQTGIDLSGCTKWWRFAEFQYLQPGPPRRLQ

421 TVVVYLPDVWTIMPTLEEWEALCQQKAAEAAPPTOEAQGETEPTEQAPDALEQAADTSRR

481 NAETPEATTQQETDTDLPEAPPPPLEPAVIARPGCVNLSLHGIVEDRRPKERISFEVMVL

541 AELFLEMLQRDFGYRVYKMLLSLPEKVVSPPEPEKEEAAKEEATKEEEAIKEEVVKEPKD

601 EAQNEGPATESEAPLKEDGLLPKPLSSGGEEEEKPRGEASEDLCEMALDPELLLLRDDGE

661 EEFAGAKLEDSEVRSVASNQSEMEFSSLQDMPKELDPSAVLPLDCLLAFVFFDANWCGYL

721 HRRDLERILLTLGIRLSAEQAKQLVSRVVTQNICQYRSLQYSRQEGLDGGLPEEVLFGNL

781 DLLPPPGKSTKPGAAPTEHKALVSHNGSLINVGSLLQRAEQQDSGRLYLENKIHTLELKL

841 EESHNRFSATEVTNKTLAAEMQELRVRLAEAEETARTAERQKSQLQRLLQELRRRLTPLQ 901 LEIQRVVEKADSWVEKEEPAPSN

\section{Predicted domains}

NLS

Leucine zipper

EF Hand

Coiled-coil

Amino acid sequence of DBC-1 (h.s.)

Accession: UniProtKB/Swiss-Prot Q8N163 (K1967_HUMAN)

Domains were predicted by PROSITE and BLAST protein homology search (Sundararajan et al., 2005) 


\section{Figure IV}

siRNA Nup358 + HA-Nup358 fragments

\begin{tabular}{|l|l|l|l|l|l|l|l|}
\hline & siRNA & $\begin{array}{l}\text { aa } \\
1-2448\end{array}$ & $\begin{array}{l}\text { aa } \\
1-2148\end{array}$ & $\begin{array}{l}\text { aa } \\
1-1810\end{array}$ & $\begin{array}{l}\text { aa } \\
1-1306\end{array}$ & $\begin{array}{l}\text { aa } \\
1-1170\end{array}$ & $\begin{array}{l}\text { aa } \\
1-1133\end{array}$ \\
\hline $\begin{array}{l}\text { Control } \\
\text { NiRNA }\end{array}$ & $<0.007$ & & & & & & $<0.008$ \\
\hline Nup358 & & $<0.007$ & $<0.03$ & $<0.04$ & $<0.0002$ & $<0.0006$ & \\
\hline
\end{tabular}

p-values for rescue experiments of GFP-DBC-1 with HA-Nup358 fragments

Control or Nup358-depleted HeLa P4 cells were cotransfected with plasmids coding for GFP-DBC-1 and either an empty vector or siRNA-resistant truncations of Nup358. For more experimental details, refer to Figure 3-18. For quantification, distribution of GFP-DBC-1 in the absence or presence of the HA-Nup358 fragments was analyzed and grouped into the three categories $\mathrm{N}>\mathrm{C}, \mathrm{N}=\mathrm{C}$ or $\mathrm{C}>\mathrm{N}$. The experiment was carried out 3-4 times. The statistical significance of the data was determined using the student's t-test, and the respective $\mathrm{p}$-value is listed in the table. 


\section{ABBREVIATIONS}

\begin{tabular}{|c|c|}
\hline$A$ & adenine (DNA and RNA) \\
\hline aа & amino acids \\
\hline Aff. pur. & affinity purified \\
\hline ATP & adenosine-5-triphosphate \\
\hline bp & basepair \\
\hline BSA & bovine serum albumine \\
\hline C & cytosine (DNA and RNA) \\
\hline cDNA & complementary DNA \\
\hline CFP & cyan fluorescent protein \\
\hline CIP & calf intestinal phosphatase \\
\hline CMV & cytomegalovirus promoter \\
\hline CRM1 & chromosome region maintenance 1 \\
\hline C-terminus & carboxy terminus \\
\hline DBC-1 & deleted in breast cancer 1 \\
\hline DDX43 & DEAD-box polypeptide 43 \\
\hline DDX59 & DEAD-box polypeptide 59 \\
\hline DMEM & Dulbeccos's modified eagles medium \\
\hline DMSO & dimethyl sulfoxide \\
\hline DNA & desoxyribonucleic acid \\
\hline dNTP & 2'-desoxynucleoside-5'-triphosphate \\
\hline DTT & dithiothreitol \\
\hline $\mathrm{ECL}$ & enhanced chemical luminescence \\
\hline E. coli & Escherichia coli \\
\hline EDTA & ethylenediaminetetraacetic acid \\
\hline EGTA & ethylene glycol tetraacetic acid \\
\hline $\mathrm{EtOH}$ & ethanol \\
\hline $\exp$ & exportin \\
\hline FG-repeat & phenylalanine glycine repeat \\
\hline FCS & fetal calf serum \\
\hline FP & fluorescent protein \\
\hline G & guanine (DNA and RNA) \\
\hline G & Gloria, name of rabbit (imp $\beta$ antibody) \\
\hline GAP & GTPase-activating protein \\
\hline
\end{tabular}




\section{General abbreviations}

\begin{tabular}{|c|c|}
\hline GDP & guanosine-5'-diphosphate \\
\hline GEF & guanine nucleotide exchange factor \\
\hline GFP & green fluorescent protein \\
\hline GR & glucocorticoid receptor \\
\hline GST & glutathione-S-transferase \\
\hline GTP & guanosine-5'-triphosphate \\
\hline GTPase & GTP-hydrolase \\
\hline $\mathrm{H}$ & Hermione, name of rabbit (imp $\beta$ antibody) \\
\hline HA & hemagglutinin \\
\hline $\mathrm{HCl}$ & hydrochloric acid \\
\hline HEPES & $\begin{array}{l}\text { [4-(2-hydroxyethyl)-1- } \\
\text { piperazine]ethanesulfonic }\end{array}$ \\
\hline His & histidine tag \\
\hline HIV & human immunodeficiency virus \\
\hline hnRNP & heterogeneous nuclear ribonucleoprotein \\
\hline IF & immunofluorescence \\
\hline $\lg G$ & immunoglobuline $\mathrm{G}$ \\
\hline imp & importin \\
\hline IP & immunoprecipitation \\
\hline IPTG & isopropyl- $\beta$-D-thiogalactopyranoside \\
\hline $\mathrm{Da}$ & dalton \\
\hline LB & Luria-Bertani \\
\hline LMB & leptomycin B (Crm1 inhibitor) \\
\hline LIFEdb & $\begin{array}{l}\text { Database for localization, interaction, } \\
\text { functional assays and expression of proteins }\end{array}$ \\
\hline MCS & multiple cloning site \\
\hline $\mathrm{mt}$ & mutant \\
\hline nct & nucleotide \\
\hline NES & nuclear export signal \\
\hline cNLS & (classical) nuclear localization signal \\
\hline No & number \\
\hline NP40 & octyl phenoxylpolyethoxylethanol \\
\hline NPC & nuclear pore complex \\
\hline N-terminus & amino terminus \\
\hline NTR & nuclear transport receptor \\
\hline Nup & nucleoporin \\
\hline ORF & open reading frame \\
\hline PAGE & polyacrylamide gel electrophoresis \\
\hline
\end{tabular}


General abbreviations

\begin{tabular}{|c|c|}
\hline PBS & phosphate buffered saline \\
\hline PCR & polymerase chain reaction \\
\hline Pefa bloc & $\begin{array}{l}\text { 4-2-aminoethyl-benzenesulfonyl fluoride } \\
\text { hydrochloride }\end{array}$ \\
\hline PKI & protein kinase inhibitor \\
\hline POM & pore membrane protein \\
\hline PY-NLS & $\begin{array}{l}\text { proline tyrosine-NLS, motif for interaction of } \\
\text { transportin }\end{array}$ \\
\hline RFP & red fluorescent protein \\
\hline $\mathrm{pH}$ & negative common logarithm of the proton \\
\hline PMSF & phenylmethylsulphonyl fluoride \\
\hline Ran & Ras related nuclear protein \\
\hline RanBP1 & Ran binding protein 1 \\
\hline RanBP2 & Ran binding protein 2 \\
\hline RanBD & Ran binding domain \\
\hline RCC1 & regulator of chromosome condensation 1 \\
\hline RNA & ribonucleic acid \\
\hline ROI & region of interest \\
\hline rpm & rotations per minute \\
\hline RNAi & RNA interference \\
\hline RNase & ribonuclease \\
\hline SDS & sodium dodecyl sulfate \\
\hline SiRNA & small interfering RNA \\
\hline Sox 6 & SRY (sex determining region Y)-box 6 \\
\hline SRY & Sex-determining region y \\
\hline SUMO & small ubiquitin related modifier \\
\hline SV40 & simian virus 40 \\
\hline $\mathrm{T}$ & thymine, in context of DNA and RNA \\
\hline TAE & Tris / Acetate / EDTA \\
\hline TAP & $\begin{array}{l}\text { Tip-(Herpesvirus saimiri tyrosine-kinase- } \\
\text { interacting-protein) associated } \\
\text { protein }\end{array}$ \\
\hline TE & Tris / EDTA \\
\hline Triton $\mathrm{X}-100$ & 4-octylphenol polyethoxylate \\
\hline Tween20 & polyoxyethylene (20) sorbitan monolaurate \\
\hline$U$ & uracile, in context of RNA \\
\hline UV & ultraviolet \\
\hline $\mathrm{v} / \mathrm{v}$ & volume per volume \\
\hline
\end{tabular}


General abbreviations

\begin{tabular}{ll}
\hline WB & western blot \\
wt & wild type \\
w/v & weight per volume
\end{tabular}

\section{Physical units}

\begin{tabular}{ll}
\hline${ }^{\circ} \mathrm{C}$ & degree celsius \\
$\mathrm{g}$ & gram \\
$\mathrm{Xg}$ & acceleration of gravity on earth \\
$\mathrm{hr}$ & hour(s) \\
$\mathrm{I}$ & liter \\
$\mathrm{M}$ & molar $(\mathrm{mol} / \mathrm{l})$ \\
$\mathrm{min}$ & minute $(\mathrm{s})$ \\
$\mathrm{m}$ & meter \\
$\mathrm{OD}$ & optical density \\
$\mathrm{pH}$ & potential hydrogen \\
$\mathrm{rpm}$ & rotations per minute \\
$\mathrm{S}$ & second(s) \\
$\mathrm{U}$ & Unit \\
$\mathrm{V}$ & volt
\end{tabular}

\section{Prefixes}

\begin{tabular}{lll}
\hline $\mathrm{k}$ & kilo- & $10^{3}$ \\
$\mathrm{c}$ & centi- & $10^{-2}$ \\
$\mathrm{~m}$ & mili- & $10^{-3}$ \\
$\mu$ & micro- & $10^{-6}$ \\
$\mathrm{n}$ & nano- & $10^{-9}$ \\
$\mathrm{p}$ & pico- & $10^{-12}$
\end{tabular}




\section{Code for amino acids}

\begin{tabular}{lll}
\hline A & Ala & alanine \\
C & Cys & cysteine \\
D & Asp & aspartate \\
E & Glu & glutamate \\
F & Phe & phenylalanine \\
G & Gly & glycine \\
H & His & histidine \\
I & Iso & isoleucine \\
K & Lys & lysine \\
L & Leu & leucine \\
M & Met & methionine \\
N & Asn & asparagine \\
P & Pro & proline \\
Q & Gln & glutamine \\
R & Arg & arginine \\
S & Ser & serine \\
T & Thr & threonine \\
V & Val & valine \\
W & Trp & tryptophane \\
Y & Tyr & tyrosine \\
X & any & \\
Z & apolar residue & \\
& &
\end{tabular}





\section{ACKNOWLEDGEMENTS}

Ein großes, herzliches Dankeschön geht an meinen Betreuer Dr. Ralph Kehlenbach: Danke für dieses spannende und herausfordernde, wenn auch nicht immer einfache Projekt, für all deine Ideen, deine permanente Unterstützung und Aufmunterung, wenn es mal wieder nicht weiterging. Ich habe viel gelernt in den letzten vier Jahren.

Für die gute Betreuung im Rahmen meines Thesis-Komitees bedanke ich mich ganz herzlich bei Herrn Prof. Dr. Ficner und Herrn Prof. Dr. Lührmann, dem ich des Weiteren für die Übernahme des Korreferats danke. Gleichfalls danke ich den Mitgliedern meines Prüfungskomitees, die sich bereit erklärt haben dieses Amt zu übernehmen und auch für das Entgegenkommen, einen Prüfungstermin zu finden.

Einen wesentlichen Beitrag zu dieser Arbeit haben all meine Kolleginnen getragen: Annegret, Christiane und Ulrike, für all die Arbeit, die so 'nebenher' anfällt und getan werden muß. Besonders dir, Annegret, danke ich für die letzten 2 Jahre, deinem super Arbeitseifer für meine vielen, nicht immer glorreichen Ideen. Inga, Steffie und Connie für euren Beitrag zu einer netten und lustigen Laborathmospähre und alles, was über Wissenschaft hinausging. Bei Nicole und Ruth bedanke ich mich für die nette Aufnahme in eurem kleinen Büro und den einen oder anderen Kaffee oder Tropfen Milch, den ich mir geliehen habe. Die fleißigen Menschen in unsrer Werkstatt und die Arbeitsgruppe Melchior will ich hierbei auch nicht vergessen, danke schön.

Ein dickes Dankeschön gilt hierbei unsrer Ehemaligen, Saskia, und nicht nur für das unermüdliche Korrekturlesen $;$; Ich habe bestimmt oft genervt mit meinen Emails und Hilferufen in den letzten Jahren.

Bei all meinen Korrekturlesern Saskia, Ralph, Tina, Marie, Erik und Herrn Dönecke möchte ich mich auch lieb bedanken, zudem Ihnen, Herr Dönecke, für die kommissarische Leitung der Biochemie I.

Einen nicht unwesentlichen Beitrag in den letzten 4 Jahren haben zudem all meine Freunde geleistet, ob nun in Göttingen, Münster, Köln oder sonst wo in der weiten Welt, wofür ich euch allen sehr, sehr dankbar bin.

Zuletzt jedoch möchte ich mich ganz lieb bei meiner Familie bedanken für eure fortwährende Unterstützung und den unermüdlichen Glauben an mich. Meiner Schwester Judith danke ich v.a. für die letzten drei Jahre hier in Göttingen, es tat so gut, dich hier zu haben. 



\title{
CURRICULUM VITAE
}

\author{
Personal Data
}

Name

Sarah Wälde

Born

10.11.1980 in Landshut/Germany

Education

08.2006 -present PhD thesis

The function of Nup358 in nucleocytoplasmic transport of proteins

Department of Biochemistry I

Center for Biochemistry and Molecular Cell Biology

Georg-August University, Goettingen

Supervisor: Dr. Ralph Kehlenbach

06.2005-03.2006 Diploma thesis

Investigating the integrity and stability of the nuclear lamina

Department of Molecular Genetics

German Cancer Research Center (DKFZ), Heidelberg

Supervisor: Dr. Harald Herrmann-Lerdon

10.2000-03.2005 Graduate and undergraduate studies: Biology

University of Muenster

06.2000 University entrance qualification: Abitur

Helmholtz Gymnasium, Bonn

11.1994-0.6.2000 Helmholtz Gymnasium, Bonn

08.1991-11.1994 Gymnasium Neustadt/Waldnaab 


\section{Research}

experience

05. - 07.2006 Internship

Stower's Institute for Medical Research, Kansas City, USA

09. $-11.2004 \quad$ Internship

Department of Molecular Genetics

German Cancer Research Center (DKFZ), Heidelberg

2003 Student research assistant

Institute of Infectiology

Center for Molecular Biology of Inflammation (ZMBE)

University of Muenster

Meetings/

Posters

12.2009 $49^{\text {th }}$ annual meeting of the 'American Society for Cell Biolog' (ASCB), San Diego, USA

Poster

09.2009 $6^{\text {th }}$ international PhD student symposium 'Horizons in Molecular Biology", Goettingen

Poster

$03.200629^{\text {th }}$ annual meeting of 'German Society for Cell Biology', Braunschweig

Poster

\section{Publications}

Wälde S, Kehlenbach RH (2010): The Part and the Whole: functions of nucleoporins in nucleocytoplasmic transport. Review. Trends in Cellular Biology. In press

Hutten S, Wälde S, Spillner C, Hauber J, Kehlenbach RH (2009): The nuclear pore component Nup 358 promotoes transportin-dependent nuclear import. J Cell Sci 122: 1100-10. 
Geiger SK, Bär H, Ehlermann P, Wälde S, Rutschow D, Zeller R, Ivandic BT, Zentgraf H, Katus HA, Herrmann H, Weichenhan D (2007): Incomplete nonsense-mediated decay of mutant lamin A/C mRNA provokes dilated cardiomyopathy and ventricular tachycardia. J Mol Med 86: 1432-40.

Waldmann I, Wälde S, Kehlenbach RH (2007): Nuclear import of c-Jun is mediated by multiple transport receptors. J Biol Chem 282: 27685-92.

Bär H, Goudeau B, Wälde S, Casteras-Simon M, Mücke N, Shatunov A, Goldberg YP, Clarke C, Hilton JL, Eymard B, Katus HA, Fardeau M, Goldfarb L, Vicart P, Herrmann H (2007): Conspicuous involvement of desmin tail mutations in diverse cardiac and skeletal myopathies. Hum Mut 28: 374-86. 